\title{
Application of Multiaxial Fatigue Analysis Methodologies for the Improvement of the Life Prediction of Landing Gear Fuse Pins
}

by

Quoc-Viet Le-The

A thesis submitted to the Faculty of Graduate and Postdoctoral Affairs in partial fulfillment of the requirements for the degree of

Master of Applied Sciences

in

Aerospace Engineering

Carleton University

Ottawa, Ontario

(C) 2016, Quoc-Viet Le-The 


\begin{abstract}
Fuse pins are used in landing gear designs to attach the landing gear to the airframe and are designed to allow for a controlled separation of the landing gear from the aircraft structure in the event of a crash. Traditional uniaxial fatigue analysis methods have been found to be insufficient for properly predicting the fatigue life of the fuse pins; often significantly over-predicting or under-predicting the fatigue life. To improve the life prediction of these pins, multiaxial fatigue analysis methods were selected and implemented into a custom fatigue analysis program. The analysis procedure includes the constitutive modeling of the elastic-plastic material, the notch correction methods, cycle counting method and the fatigue damage criteria. The results of predictions made using the multiaxial fatigue methods for three fuse pin designs were compared to data from fatigue tests of three different landing gear assemblies. It was found that the performance of the constitutive model used for predicting the elastic and plastic stresses and strains, and the choice of fatigue damage criterion had the most effect on the final predicted fatigue life. Significant improvements over the traditional uniaxial fatigue analysis methods were shown and reasonable correlation with test data was demonstrated using the multiaxial approaches.
\end{abstract}




\section{Acknowledgements}

I would like to thank Professor Xin Wang, my thesis supervisor, for all of his support and encouragement with this project. He was quick to help me get this project started well before the start of my graduate studies. I am grateful for the open environment that he provided me with to develop my own ideas while providing the guidance that I needed to refine my project.

Brad Baird and Paul Vanderpol at UTC Aerospace Systems in Oakville have been exceptionally supportive of my project since the beginning. My work with UTC Aerospace Systems as a coop student inspired the initial idea for the project. I am very grateful for their dedication to the project and their feedback on my work.

The scholarships and research grants from Carleton University to support my research and this project are also greatly appreciated.

Finally, I would like to thank my family and friends for their support and encouragement during my graduate studies. More specifically, I am very grateful for the patience of my wife during these busy times. 


\section{Table of Contents}

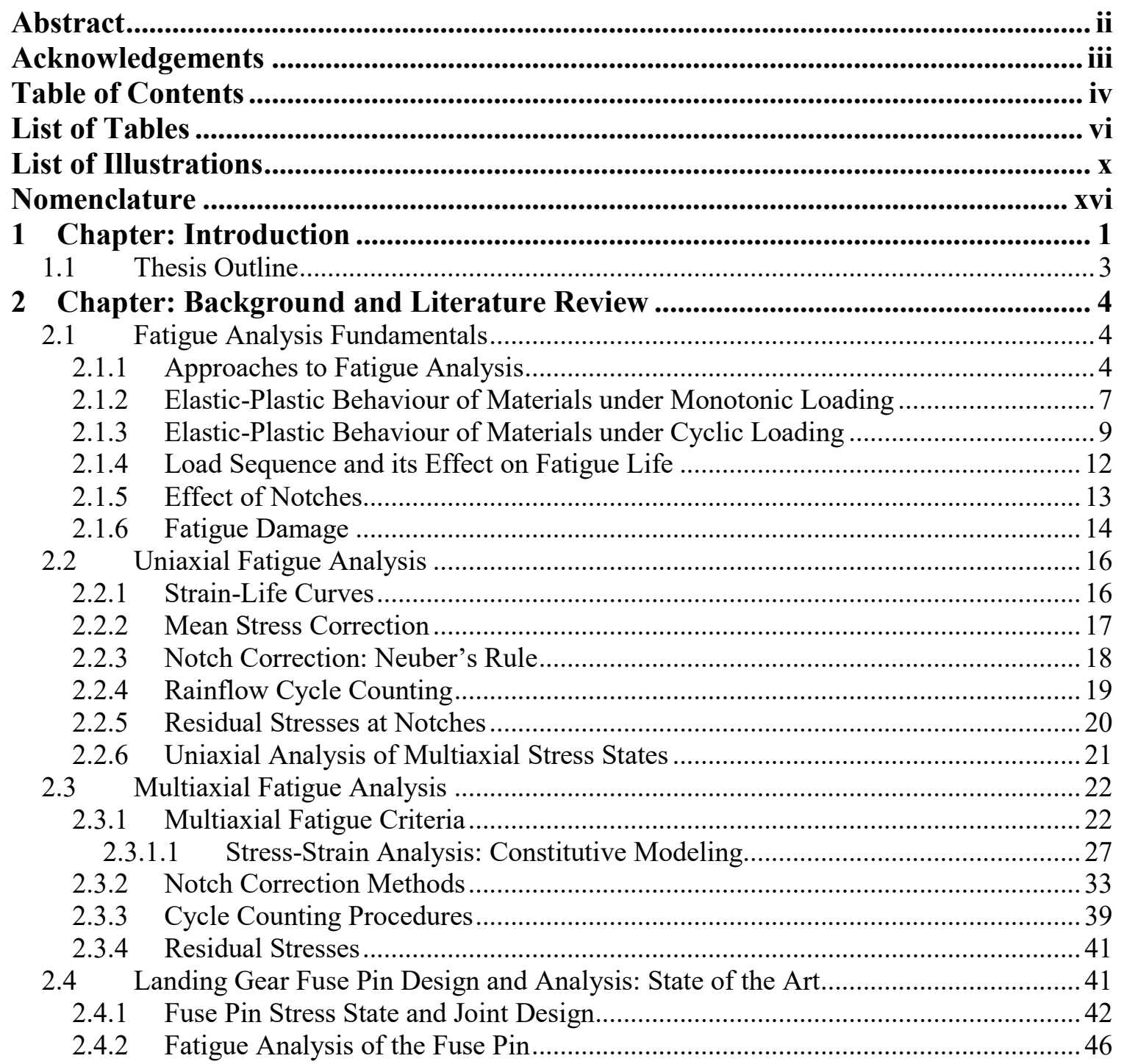

3 Chapter: Multiaxial Constitutive Model .................................................................. 48

3.1 Implementation of the Jiang-Sehitoglu Incremental Plasticity Model ........................ 48

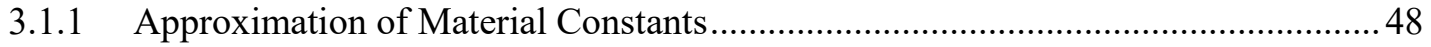

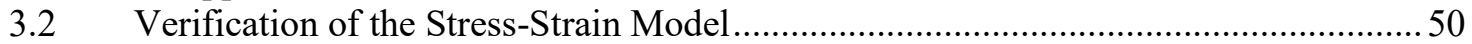

4 Chapter: Multiaxial Notch Correction Methods .................................................... 55

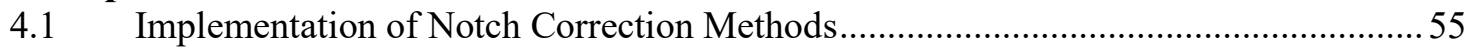

4.2 Verification of Proportional Loading of Notch Specimens ........................................5

4.2.1.1 Finite Element Analysis of Notched Specimens ............................................5 57

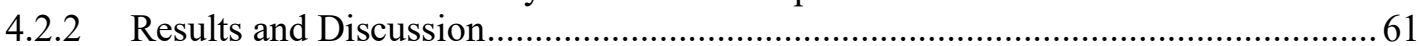

4.3 Verification of Non-proportional Loading of Notched Specimens .............................. 74

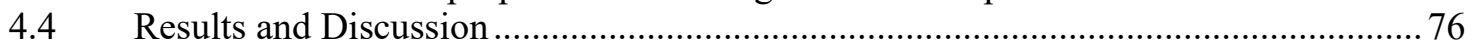

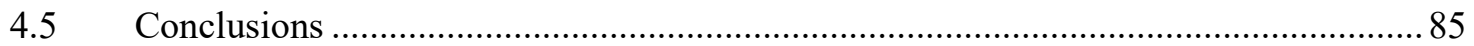

5 Chapter: Multiaxial Cycle Counting Procedures ................................................... 86

$5.1 \quad$ Example of Wang-Brown Cycle Count.................................................................... 86

5.2 Implementation of the Bannantine-Socie Cycle Counting Procedure .......................... 93

5.3 Comparison of Wang-Brown and Bannantine-Socie Counting Procedures ................. 94 
5.4 Modification of Wang-Brown Cycle Counting Procedure ..........................................94

6 Chapter: Fatigue Analysis of Test Fuse Pins..................................................... 98

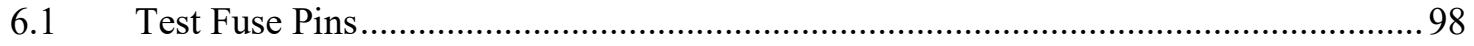

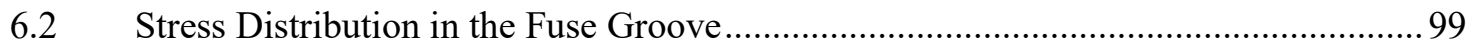

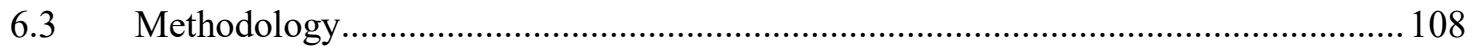

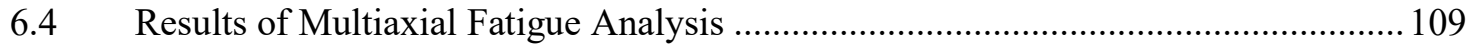

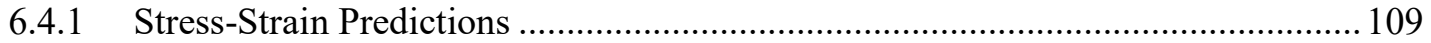

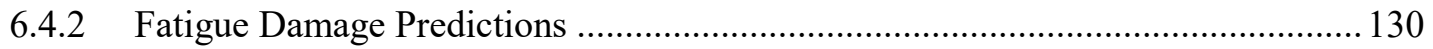

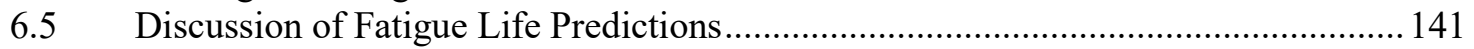

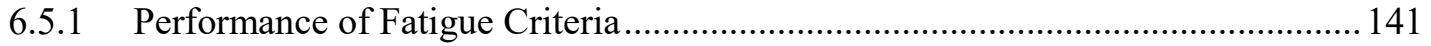

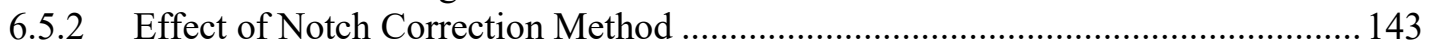

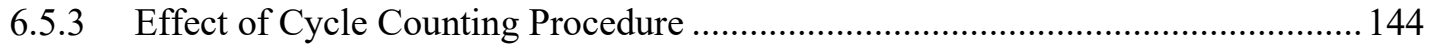

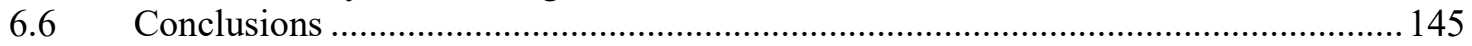

Chapter 7 : Conclusions and Recommendations ................................................... 147

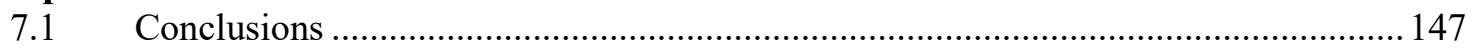

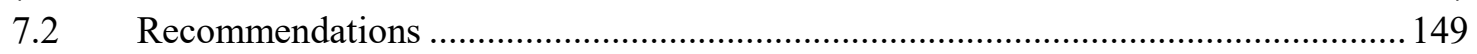

Bibliography ................................................................................................................................. 151

Appendices.......................................................................................................................................... 156

Appendix A Fatigue Damage Results Calculated using Multiaxial Fatigue Approach........... 156

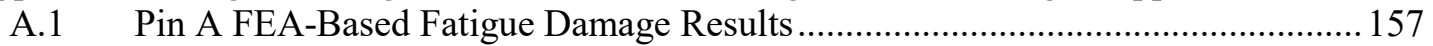

A.2 Pin A Test-Based Fatigue Damage Results...................................................... 170

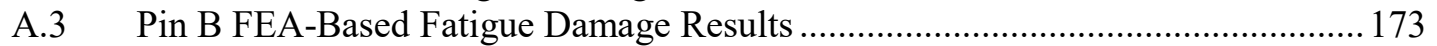

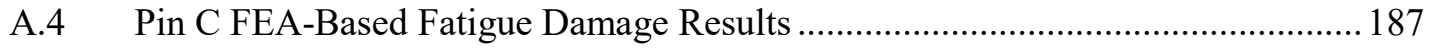

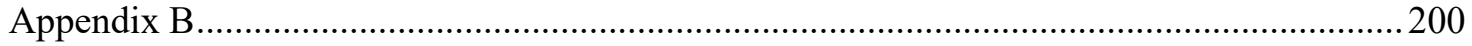

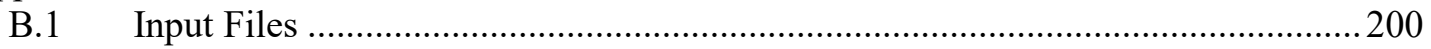

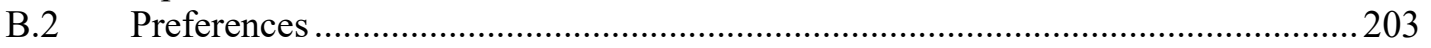

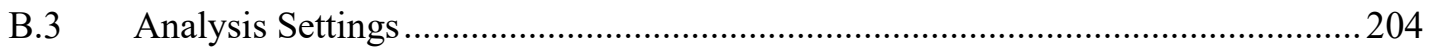

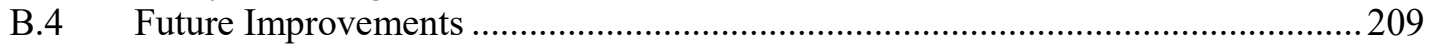




\section{List of Tables}

Table 3-1: Summary of Stress-Strain Model Verification Tests 50

Table 3-2: Stress inputs for Stress-Strain Model Verification Tests (stresses in MPa) 51

Table 3-3: Material properties for 304L stainless steel (Shamsaei, Fatemi, \& Socie, 2010).

Table 4-1: Geometry of specimens used for verification of multiaxial notch analysis. 58

Table 4-2: Material properties for SAE 1070 (Firat, 2011) and SAE 1045 (Singh, Glinka, \& Dubey, 1996).

Table 4-3: End loads applied to finite element analyses for notch verification tests.

Table 4-4: Elastic notch stresses (MPa) used as input into stress-strain model for notch verification tests.

Table 4-6: Strain range prediction comparisons for non-proportional loading verification tests.

Table 5-1:Load case pairs determined using the Wang-Brown cycle counting procedure on the sample axial-torsion strain data from Wang \& Brown (1996). 88

Table 6-1: Summary fuse pins provided by UTAS-LS 98

Table 6-2: Summary of categories of the different elements of fatigue analysis. $\quad 108$

Table 6-3: Fatigue damages calculated by UTAS-LS for the three test fuse pins using three different fatigue analysis methodologies.

131

Table A.1-1: Fatigue damage results for Pin A at the neutral axis and centre of the fuse groove $(\hat{\mathrm{z}}=0)$.

Table A.1-2: Fatigue damage results for Pin A at $45^{\circ}$ to the neutral axis and centre of the fuse groove $(\hat{z}=0)$.

Table A.1-3: Fatigue damage results for Pin A at $45^{\circ}$ to the neutral axis and offset $19 \%$ from the centre of the fuse groove $(\hat{z}=0.19)$.

Table A.1-4: Fatigue damage results for Pin A at $45^{\circ}$ to the neutral axis and offset $-19 \%$ from the centre of the fuse groove $(\hat{z}=-0.19)$.

Table A.1-5: Fatigue damage results for Pin A at $90^{\circ}$ to the neutral axis and at the centre of the fuse groove $(\hat{z}=0)$. 
Table A.1-6: Fatigue damage results for Pin A at $90^{\circ}$ to the neutral axis and offset $21 \%$ from the centre of the fuse groove $(\hat{z}=0.21)$.

Table A.1-7: Fatigue damage results for Pin A at $90^{\circ}$ to the neutral axis and offset $-21 \%$ from the centre of the fuse groove $(\hat{z}=-0.21)$.

Table A.1-8: Fatigue damage results for Pin A at $-45^{\circ}$ to the neutral axis and at the centre of the fuse groove $(\hat{z}=0)$.

Table A.1-9: Fatigue damage results for Pin A at $-45^{\circ}$ to the neutral axis and offset $19 \%$ from the centre of the fuse groove $(\hat{z}=0.19)$.

Table A.1-10: Fatigue damage results for Pin A at $-45^{\circ}$ to the neutral axis and offset $-19 \%$ from the centre of the fuse groove $(\hat{z}=-0.19)$.

166

Table A.1-11: Fatigue damage results for Pin A at $-90^{\circ}$ to the neutral axis and at the centre of the fuse groove $(\hat{\mathrm{z}}=0)$.

Table A.1-12: Fatigue damage results for Pin A at $-90^{\circ}$ to the neutral axis and offset $21 \%$ from the centre of the fuse groove $(\hat{z}=21 \%)$.

Table A.1-13: Fatigue damage results for Pin A at $-90^{\circ}$ to the neutral axis and offset $-21 \%$ from the centre of the fuse groove $(\hat{z}=-21 \%)$.

Table A.2-1: Fatigue damage results for Pin A at the neutral axis and centre of the fuse groove $(\hat{z}=0)$. Input stresses calculated using strain test data.

Table A.2-2: Fatigue damage results for Pin A at $45^{\circ}$ to the neutral axis and centre of the fuse groove $(\hat{z}=0)$.

Table A.2-3: Fatigue damage results for Pin A at $90^{\circ}$ to the neutral axis and at the centre of the fuse groove $(\hat{z}=0)$.

172

Table A.3-1: Fatigue damage results for Pin B at the neutral axis and centre of the fuse groove $(\hat{\mathrm{z}}=0)$. Input stresses determined from elastic FEA data.

Table A.3-2: Fatigue damage results for Pin B at $45^{\circ}$ to the neutral axis and at the centre of the fuse groove $(\hat{\mathrm{z}}=0)$. Input stresses determined from elastic FEA data.

Table A.3-3: Fatigue damage results for Pin B at $45^{\circ}$ to the neutral axis and offset $35 \%$ from the centre of the fuse groove $(\hat{z}=0.35)$. Input stresses determined from elastic FEA data.

Table A.3-4: Fatigue damage results for Pin B at $45^{\circ}$ to the neutral axis and offset $21 \%$ from the centre of the fuse groove $(\hat{\mathrm{z}}=-0.21)$. Input stresses determined from elastic FEA data. 
Table A.3-5: Fatigue damage results for Pin B at $90^{\circ}$ to the neutral axis and at the centre of the fuse groove $(\hat{z}=0)$. Input stresses determined from elastic FEA data.

178

Table A.3-6: Fatigue damage results for Pin B at $90^{\circ}$ to the neutral axis and offset $26 \%$ from the centre of the fuse groove $(\hat{z}=0.26)$. Input stresses determined from elastic FEA data.

Table A.3-7: Fatigue damage results for Pin B at $90^{\circ}$ to the neutral axis and offset $-23 \%$ from the centre of the fuse groove $(\hat{z}=-0.23)$. Input stresses determined from elastic FEA data.

Table A.3-8: Fatigue damage results for Pin B at $-45^{\circ}$ to the neutral axis and at the centre of the fuse groove $(\hat{z}=0)$. Input stresses determined from elastic FEA data.

181

Table A.3-9: Fatigue damage results for Pin B at $-45^{\circ}$ to the neutral axis and offset $35 \%$ from the centre of the fuse groove $(\hat{\mathrm{z}}=0.35)$. Input stresses determined from elastic FEA data.

Table A.3-10: Fatigue damage results for Pin B at $-45^{\circ}$ to the neutral axis and offset $21 \%$ from the centre of the fuse groove $(\hat{z}=-0.21)$. Input stresses determined from elastic FEA data.

Table A.3-11: Fatigue damage results for Pin $\mathrm{B}$ at $-90^{\circ}$ to the neutral axis and at the centre of the fuse groove $(\hat{z}=0)$. Input stresses determined from elastic FEA data.

184

Table A.3-12: Fatigue damage results for Pin B at $-90^{\circ}$ to the neutral axis and offset $26 \%$ from the centre of the fuse groove $(\hat{z}=0.26)$. Input stresses determined from elastic FEA data.

Table A.3-13: Fatigue damage results for Pin B at $-90^{\circ}$ to the neutral axis and offset $-23 \%$ from the centre of the fuse groove $(\hat{z}=-0.23)$. Input stresses determined from elastic FEA data.

Table A.4-1: Fatigue damage results for Pin $\mathrm{C}$ at the neutral axis and centre of the fuse groove $(\hat{\mathrm{z}}=0)$. Input stresses determined from elastic FEA data.

Table A.4-2: Fatigue damage results for Pin $\mathrm{C}$ at $45^{\circ}$ to the neutral axis and at the centre of the fuse groove $(\hat{z}=0)$. Input stresses determined from elastic FEA data.

Table A.4-3: Fatigue damage results for Pin $\mathrm{C}$ at $45^{\circ}$ to the neutral axis and offset $28 \%$ from the centre of the fuse groove $(\hat{\mathrm{z}}=0.28)$. Input stresses determined from elastic FEA data.

Table A.4-4: Fatigue damage results for Pin $\mathrm{C}$ at $45^{\circ}$ to the neutral axis and offset $28 \%$ from the centre of the fuse groove $(\hat{\mathrm{z}}=-0.28)$. Input stresses determined from elastic FEA data. 
Table A.4-5: Fatigue damage results for Pin $\mathrm{C}$ at $90^{\circ}$ to the neutral axis and at the centre of the fuse groove $(\hat{z}=0)$. Input stresses determined from elastic FEA data.

Table A.4-6: Fatigue damage results for Pin $\mathrm{C}$ at $90^{\circ}$ to the neutral axis and offset $30 \%$ from the centre of the fuse groove $(\hat{\mathrm{z}}=0.30)$. Input stresses determined from elastic FEA data.

Table A.4-7: Fatigue damage results for Pin $\mathrm{C}$ at $90^{\circ}$ to the neutral axis and offset $-30 \%$ from the centre of the fuse groove $(\hat{z}=-0.30)$. Input stresses determined from elastic FEA data.

Table A.4-8: Fatigue damage results for Pin $\mathrm{C}$ at $-45^{\circ}$ to the neutral axis and at the centre of the fuse groove $(\hat{z}=0)$. Input stresses determined from elastic FEA data.

Table A.4-9: Fatigue damage results for Pin $\mathrm{C}$ at $-45^{\circ}$ to the neutral axis and offset $28 \%$ from the centre of the fuse groove $(\hat{\mathrm{z}}=0.28)$. Input stresses determined from elastic FEA data.

Table A.4-10: Fatigue damage results for Pin $\mathrm{C}$ at $-45^{\circ}$ to the neutral axis and offset $-28 \%$ from the centre of the fuse groove $(\hat{\mathrm{z}}=-0.28)$. Input stresses determined from elastic FEA data.

Table A.4-11: Fatigue damage results for Pin $\mathrm{C}$ at $-90^{\circ}$ to the neutral axis and at the centre of the fuse groove $(\hat{z}=0)$. Input stresses determined from elastic FEA data.

Table A.4-12: Fatigue damage results for Pin $\mathrm{C}$ at $-90^{\circ}$ to the neutral axis and offset $30 \%$ from the centre of the fuse groove $(\hat{\mathrm{z}}=0.30)$. Input stresses determined from elastic FEA data.

Table A.4-13: Fatigue damage results for Pin $\mathrm{C}$ at $-90^{\circ}$ to the neutral axis and offset $-30 \%$ from the centre of the fuse groove $(\hat{z}=-0.30)$. Input stresses determined from elastic FEA data. 


\section{List of Illustrations}

Figure 1-1: Schematic representation of stress-life and strain-life curve approximations . 7

Figure 1-2: Schematic representation of kinematic hardening and the Bauschinger effect.

Figure 1-3: Schematic representation of isotropic hardening........................................ 10

Figure 1-4: Example of stabilization of hysteresis loop under strain-controlled reversed loading. 11

Figure 1-5: Schematic of notch stresses...................................................................... 14

Figure 2-6: Illustration of cycle counting terminology .................................................... 20

Figure 1-7: General procedure for implementing multiaxial notch correction methods. . 38

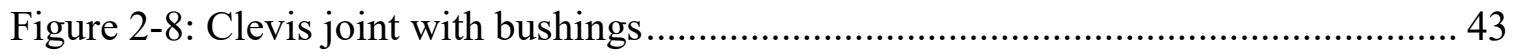

Figure 2-9: Cantilever joint with bushings ............................................................... 44

Figure 2-10: Cantilever joint with spherical bearing ................................................... 45

Figure 2-11: General triaxial stress state of the fuse pin groove. ...................................... 46

Figure 3-1: Illustration of discretization of $90^{\circ}$ out-of-phase stress path in the $\sigma x-3 \tau x y$

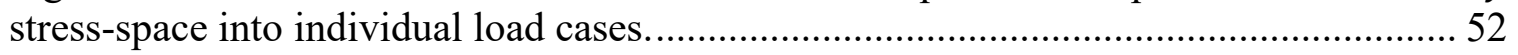

Figure 3-2: Stress-strain model verification test results compared to experimental cyclic stress-strain curves for 304L stainless steel from Shamsaei et al. (2010). ....................... 53

Figure 3-3: Convergence of axial and shear strain components in $90^{\circ} \mathrm{OP}$ cyclic loading

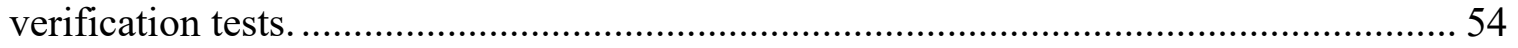

Figure 4-1: Geometry of notched specimen for numerical analysis............................... 57

Figure 4-2: Axisymmetric finite element analysis of axial-torsion specimens................. 60

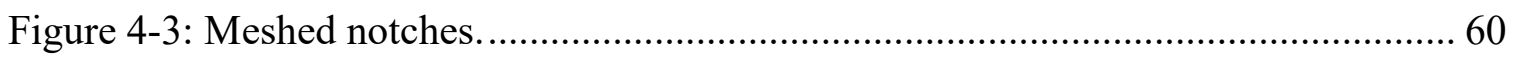

Figure 4-4: Comparison of notch correction and FEA stress predictions for Specimen A

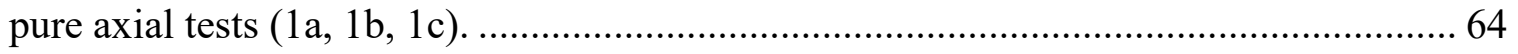

Figure 4-5: Comparison of notch correction and FEA stress predictions for Specimen B

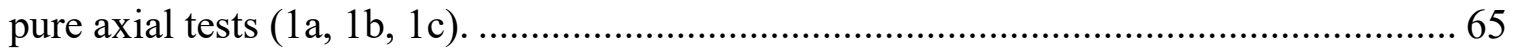

Figure 4-6: Comparison of notch correction and FEA stress predictions for Specimen C

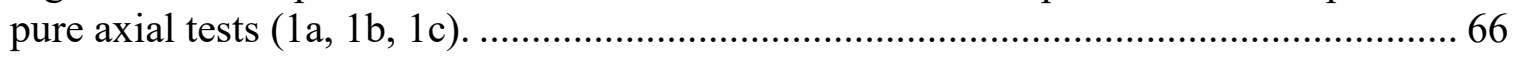


Figure 4-7: Comparison of notch correction and FEA stress predictions for Specimen A

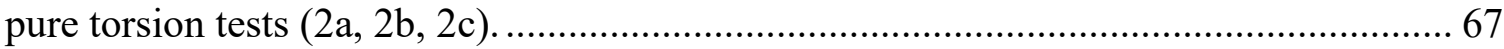

Figure 4-8: Comparison of notch correction and FEA stress predictions for Specimen B pure torsion tests $(2 \mathrm{a}, 2 \mathrm{~b}, 2 \mathrm{c})$. 68

Figure 4-9: Comparison of notch correction and FEA stress predictions for Specimen C pure torsion tests $(2 \mathrm{a}, 2 \mathrm{~b}, 2 \mathrm{c})$.

Figure 4-10: Comparison of notch correction and FEA stress predictions for Specimen A axial-torsion tests $(3 \mathrm{a}, 3 \mathrm{~b}, 3 \mathrm{c})$. 70

Figure 4-11: Comparison of notch correction and FEA stress predictions for Specimen B axial-torsion tests (3a, 3b, 3c). 71

Figure 4-12: Comparison of notch correction and FEA stress predictions for Specimen C axial-torsion tests (3a, 3b, 3c). 72

Figure 4-13: Ratio of plastic zone size to specimen groove radius for Test 1a (400 MPa),

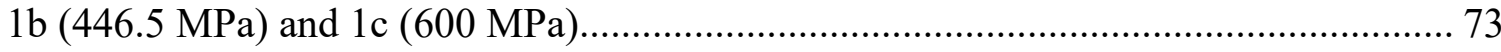

Figure 4-14: Box-patterned stress path used to test cyclic non-proportional loading. ..... 75

Figure 4-15: Unequal frequency stress path used to test cyclic non-proportional loading. 75

Figure 4-16: Strain predictions using incremental ESED notch correction for box-patterned stress path compared to experimental data for SAE 1070 (Ince, Glinka, \& Buczynski, 2014). 78

Figure 4-17: Strain predictions using the multiaxial Neuber's rule notch correction for boxpatterned stress path compared to experimental data for SAE 1070 (Ince, Glinka, \& Buczynski, 2014). 79

Figure 4-18: Strain predictions using Unified notch correction for box-patterned stress path compared to experimental data for SAE 1070 (Ince, Glinka, \& Buczynski, 2014). ........ 80

Figure 4-19: Strain predictions using ESED notch correction for unequal frequency stress path compared to experimental data for SAE 1070 (Ince, Glinka, \& Buczynski, 2014).. 81

Figure 4-20: Strain predictions using Neuber notch correction for unequal frequency stress path compared to experimental data for SAE 1070 (Ince, Glinka, \& Buczynski, 2014).. 82

Figure 4-21: Strain predictions using Unified notch correction for unequal frequency stress path compared to experimental data for SAE 1070 (Ince, Glinka, \& Buczynski, 2014).. 83

Figure 5-1: Illustration of algorithm used for the Wang-Brown cycle counting procedure. 
Figure 5-2: Sample strain data used to test multiaxial cycle counting procedures (Wang \&

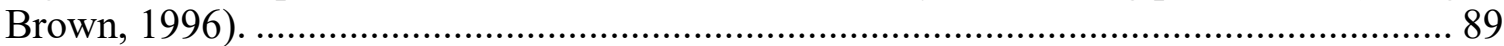

Figure 5-3: Equivalent strain history and equivalent relative strain history with respect to point A calculated in the first iteration of cycle count. 90

Figure 5-4: Equivalent relative strain histories with respect to point B and D calculated in the second iteration of cycle count. 91

Figure 5-5: Equivalent relative strain histories with respect to point $\mathrm{G}$ and $\mathrm{H}$ calculated in the third iteration of cycle count. 92

Figure 5-6: User controls for Bannantine-Socie cycle counting procedure. 93

Figure 5-7: Axial stress range of cycles counted by Wang-Brown and Bannantine-Socie counting procedures. 96

Figure 5-8: Shear stress range of cycles counted by Wang-Brown and Bannantine-Socie counting procedures 97

Figure 6-1: Hoop stress distribution along fuse pin groove at (a) neutral axis; (b) $45^{\circ}$ from neutral axis; (c) $90^{\circ}$ from neutral axis; (d) $-45^{\circ}$ from neutral axis; and (e) $-90^{\circ}$ from neutral axis. Stresses determined from elastic-plastic FEA. 102

Figure 6-2: Longitudinal stress distribution along fuse pin groove at (a) neutral axis; (b) $45^{\circ}$ from neutral axis; (c) $90^{\circ}$ from neutral axis; (d) $-45^{\circ}$ from neutral axis; and (e) $-90^{\circ}$ from neutral axis. Stresses determined from elastic-plastic FEA. 104

Figure 6-3: Shear stress distribution along fuse pin groove at (a) neutral axis; (b) $45^{\circ}$ from neutral axis; (c) $90^{\circ}$ from neutral axis; (d) $-45^{\circ}$ from neutral axis; and (e) $-90^{\circ}$ from neutral axis. Stresses determined from elastic-plastic FEA. 106

Figure 6-4: Longitudinal strain predicted by Jiang-Sehitoglu stress-strain model with suppressed non-proportional hardening and ESED notch correction compared to experimental longitudinal strain at the neutral axis and center of the fuse groove Pin A.

Figure 6-5: Hoop strain predicted by Jiang-Sehitoglu stress-strain model with suppressed non-proportional hardening and ESED notch correction compared to experimental hoop strain at the neutral axis and center of the fuse groove for Pin A. 113

Figure 6-6: Shear strain predicted by Jiang-Sehitoglu stress-strain model with suppressed non-proportional hardening and ESED notch correction compared to experimental shear strain at the neutral axis and center of the fuse groove for Pin A. 114

Figure 6-7: Longitudinal strain predicted by Jiang-Sehitoglu stress-strain model with suppressed non-proportional hardening and Neuber notch correction compared to 
experimental longitudinal strain at the neutral axis and center of the fuse groove for Pin A.

Figure 6-8: Hoop strain predicted by Jiang-Sehitoglu stress-strain model with suppressed non-proportional hardening and Neuber notch correction compared to experimental hoop strain at the neutral axis and center of the fuse groove for Pin A. 116

Figure 6-9: Shear strain predicted by Jiang-Sehitoglu stress-strain model with suppressed non-proportional hardening and Neuber notch correction compared to experimental shear strain at the neutral axis and center of the fuse groove for Pin A. 117

Figure 6-10: Longitudinal strain predicted by Jiang-Sehitoglu stress-strain model with suppressed non-proportional hardening and Unified notch correction compared to experimental longitudinal strain at the neutral axis and center of the fuse groove for Pin A.

Figure 6-11: Hoop strain predicted by Jiang-Sehitoglu stress-strain model with suppressed non-proportional hardening and Unified notch correction compared to experimental hoop strain at the neutral axis and center of the fuse groove for Pin A. 119

Figure 6-12: Shear strain predicted by Jiang-Sehitoglu stress-strain model with suppressed non-proportional hardening and Unified notch correction compared to experimental shear strain at the neutral axis and center of the fuse groove for Pin A. 120

Figure 6-13: Longitudinal, hoop, and shear strain predicted by Jiang-Sehitoglu stress-strain model with suppressed non-proportional hardening and ESED notch correction compared to experimental strain at the neutral axis and center of the fuse groove for Pin B. 121

Figure 6-14: Longitudinal, hoop, and shear strain predicted by Jiang-Sehitoglu stress-strain model with suppressed non-proportional hardening and Neuber notch correction compared to experimental strain at the neutral axis and center of the fuse groove for Pin B........ 122

Figure 6-15: Longitudinal, hoop, and shear strain predicted by Jiang-Sehitoglu stress-strain model with suppressed non-proportional hardening and Unified notch correction compared to experimental strain at the neutral axis and center of the fuse groove for Pin B. ....... 123

Figure 6-16: Longitudinal stress as predicted by linear elastic FEA and by experimental strain data for Pin A. Stress values have been normalized with respect to the maximum average shear stress in the fuse groove. 124

Figure 6-17: Hoop stress as predicted by linear elastic FEA and by experimental strain data for Pin A. Stress values have been normalized with respect to the maximum average shear stress in the fuse groove. 125

Figure 6-18: Shear stress as predicted by linear elastic FEA and by experimental strain data for Pin A. Stress values have been normalized with respect to the maximum average shear stress in the fuse groove. 126 
Figure 6-19: Longitudinal stress as predicted by linear elastic FEA and by experimental strain data for Pin B. Stress values have been normalized with respect to the maximum average shear stress in the fuse groove. 127

Figure 6-20: Hoop stress as predicted by linear elastic FEA and by experimental strain data for Pin B. Stress values have been normalized with respect to the maximum average shear stress in the fuse groove. 128

Figure 6-21: Shear stress as predicted by linear elastic FEA and by experimental strain data for Pin B. Stress values have been normalized with respect to the maximum average shear stress in the fuse groove. 129

Figure 6-22: Fatigue damage predictions using FEA-based load factors for Pin A at the neutral axis and at the centre of the fuse groove. 134

Figure 6-23: Fatigue damage predictions using FEA-based load factors for Pin A at $45^{\circ}$ to the neutral axis and at a $19 \%$ offset from the centre of the fuse groove. 135

Figure 6-24: Fatigue damage predictions using FEA-based load factors for Pin A at $-45^{\circ}$ to the neutral axis and at a $-19 \%$ offset from the centre of the fuse groove. 136

Figure 6-25: Fatigue damage predictions using FEA-based load factors for Pin B at the neutral axis and at the centre of the fuse groove. 137

Figure 6-26: Fatigue damage predictions using FEA-based load factors for Pin B at $-45^{\circ}$ to the neutral axis and at a $-21 \%$ offset to the centre of the fuse groove. 138

Figure 6-27: Fatigue damage predictions using FEA-based load factors for Pin B at $-90^{\circ}$ to the neutral axis and at a $-23 \%$ offset to the centre of the fuse groove. 139

Figure 6-28: Fatigue damage predictions using FEA-based load factors for Pin $\mathrm{C}$ at the neutral axis and at centre of the fuse groove. 140

Figure B.1-1: Load spectrum input file format for Multiaxial Fatigue v. 1.0 .............. 201

Figure B.1-2: Stress input file format for Multiaxial Fatigue v. 1.0 ............................ 201

Figure B.1-3: Material data input file format for Multiaxial Fatigue v. 1.0 ................. 202

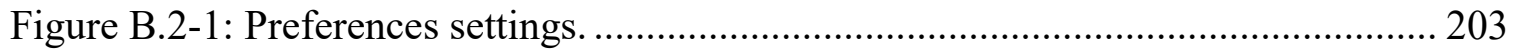

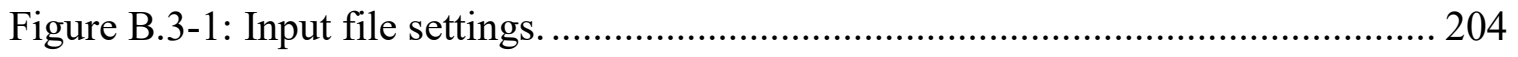

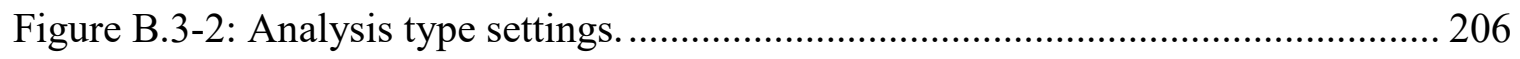

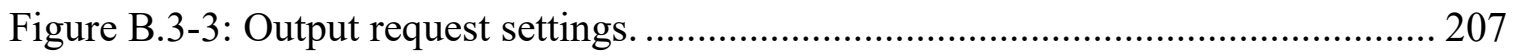

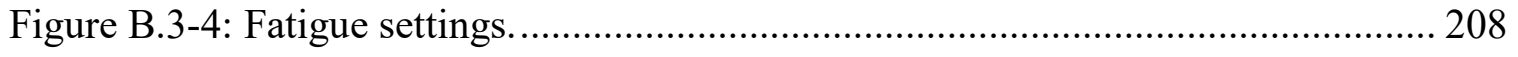


Figure B.3-5: Batch analysis settings. 


\section{Nomenclature}

A Tanaka's non-proportionality constant

$\underline{\alpha} \quad$ Backstress tensor

$d \underline{\alpha}^{i} \quad$ Change in the $\mathrm{i}^{\text {th }}$ component of the backstress tensor

BS Bannantine-Socie (cycle counting procedure)

b Fatigue strength exponent

$b_{\gamma} \quad$ Torsional fatigue strength exponent

$\beta \quad$ Non-proportionality hardening rate constant

c Fatigue ductility exponent

$c^{i} \quad$ Jiang-Sehitoglu kinematic hardening material constant

$c_{\gamma} \quad$ Torsional fatigue ductility exponent

$C_{i j k l} \quad$ Fourth-order tensor used to track non-proportional hardening

$C_{q} \quad$ Energy dissipation coefficient

$\gamma_{i j} \quad \mathrm{i}-\mathrm{j}^{\text {th }}$ shear strain component

$\frac{\Delta \gamma}{2} \quad$ Total shear strain amplitude

$\frac{\Delta \gamma_{\max }}{2} \quad$ Maximum shear strain amplitude

$\delta_{i j} \quad$ Kronecker delta

D Cumulative fatigue damage

E Young's modulus of elasticity

ESED Equivalent strain energy density

$\varepsilon_{i j}^{e} \quad$ Elastic strain component

$\varepsilon_{i j}^{p} \quad$ Elastic strain component

$\varepsilon_{i i} \quad i^{\text {th }}$ normal strain component

$\varepsilon_{i j}^{N} \quad$ Elastic strain component

$\varepsilon_{e q} \quad$ Total equivalent strain

$\varepsilon_{f}^{\prime} \quad$ Fatigue ductility coefficient

$\frac{\Delta \varepsilon}{2} \quad$ Total strain amplitude

$\frac{\Delta \varepsilon_{e}}{2} \quad$ Elastic strain amplitude 


\begin{tabular}{|c|c|}
\hline$\frac{\Delta \varepsilon_{p}}{2}$ & Plastic strain amplitude \\
\hline$\frac{\Delta \varepsilon_{n}}{2}$ & Normal strain amplitude \\
\hline$\frac{\Delta \varepsilon_{1}}{2}$ & Principal strain amplitude \\
\hline$d \underline{\varepsilon}^{p}$ & Plastic strain increment tensor \\
\hline FS & Fatemi-Socie (fatigue damage criterion) \\
\hline$G$ & Shear modulus \\
\hline$h$ & Plastic modulus \\
\hline KBM & Kandil-Brown Miller (fatigue damage criterion) \\
\hline $\mathrm{K}$ & Monotonic strength coefficient \\
\hline$K^{\prime}$ & Cyclic strength coefficient \\
\hline$K_{t}$ & Theoretical stress concentration factor \\
\hline$K_{\sigma}$ & Stress concentration factor \\
\hline$K_{\varepsilon}$ & Strain concentration factor \\
\hline$K_{t, a x}$ & Theoretical axial stress concentration factor \\
\hline$K_{t, t}$ & Theoretical torsional stress concentration factor \\
\hline$k$ & FS material constant \\
\hline$k$ & Yield stress \\
\hline$L^{i}$ & Backstress unit tensor \\
\hline $\mathrm{n}$ & Monotonic strain hardening exponent \\
\hline$n^{\prime}$ & Cyclic strain hardening exponent \\
\hline$n_{i}$ & Number of cycles for the $i^{\text {th }}$ constant stress amplitude block \\
\hline$\underline{n}$ & Exterior unit normal to the yield surface at the loading point \\
\hline$N_{f i}$ & Number of cycles to failures for the $\mathrm{i}^{\text {th }}$ constant stress amplitude block \\
\hline$v$ & Poisson's ratio \\
\hline$v_{e}$ & Elastic Poisson's ratio \\
\hline$v_{p}$ & Elastic Poisson's ratio \\
\hline$\Phi$ & Non-proportionality factor \\
\hline$d p$ & Magnitude of plastic strain increment \\
\hline$r^{i}$ & Jiang-Sehitoglu kinematic hardening material constant \\
\hline
\end{tabular}




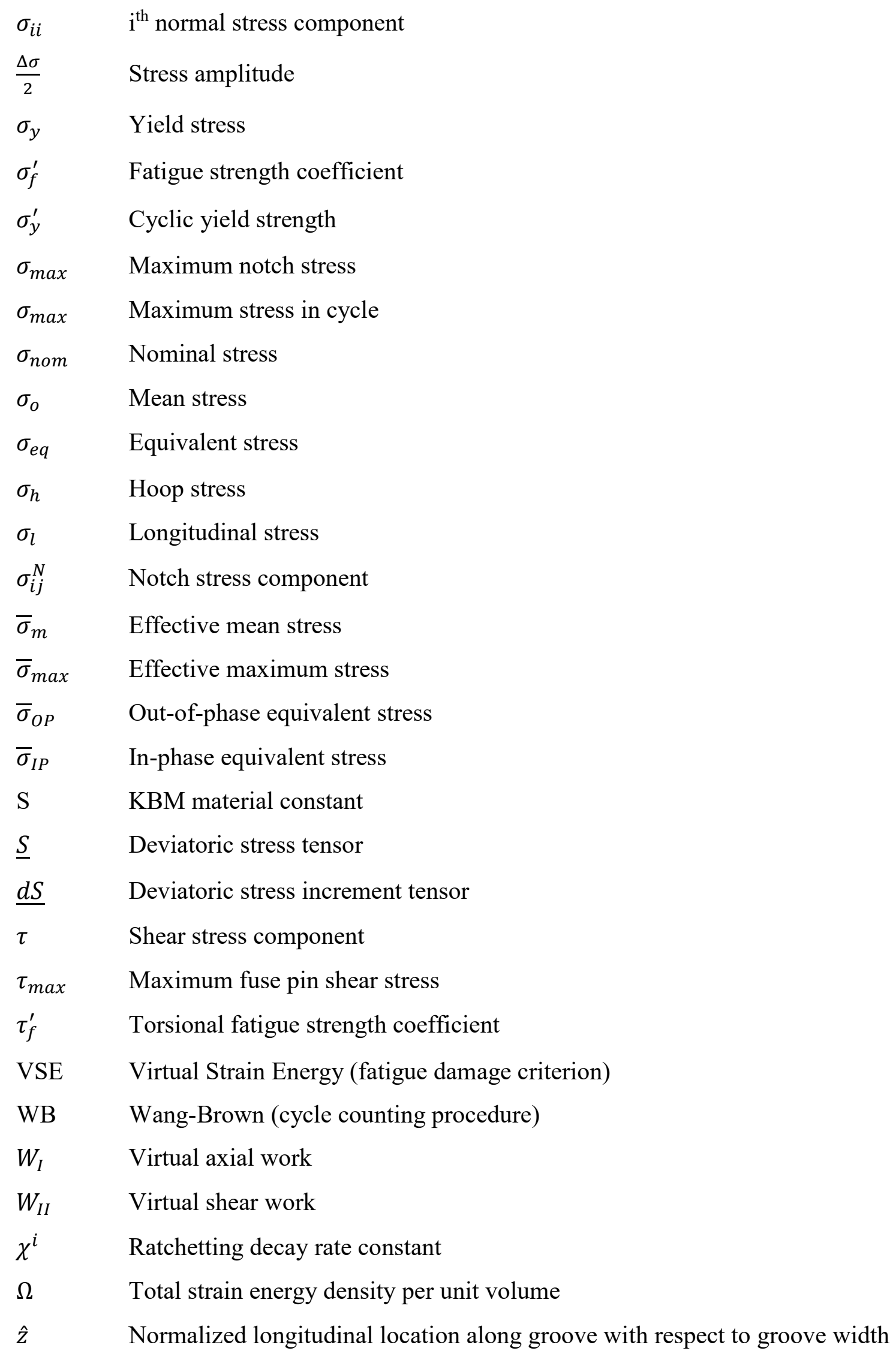




\section{Chapter: Introduction}

Engineers rely on fatigue analyses to guarantee the safe operation of components during their intended service life. In some cases, conflicting design requirements and limited analysis methods can make it difficult to achieve safe operation for a component's full service life.

In the design of aircraft landing gears, fuse pins are used to attach the landing gear to the aircraft. These fuse pins are designed so that they fail at a specific maximum load, separating the landing gear from the aircraft structure to protect the contents of the aircraft. To achieve such a controlled failure, circumferential grooves are machined into the inner diameter of fuse pins at the location of expected maximum shear force. While the driving design requirement for fuse pins is the controlled static failure of the pins, the fatigue analysis of the pins have proved to be a significant challenge for designers. Due to the complex stresses in the fuse groove due to the nature of the pin joint and the stress concentrating effect of the groove, traditional uniaxial fatigue analysis methods have been unable to properly predict the fatigue damages in the fuse pins (Narayan, Behdinan, \& Vanderpol, 2006). As a result of this shortfall in the fatigue analysis process, the replacement of fuse pins is often required earlier in the service life of the designed landing gear assembly.

To better take into account the true nature of fatigue failure under complex multiaxial stresses, multiaxial approaches to fatigue analysis have been proposed over the last few decades. While research in multiaxial fatigue analysis has existed since the early 1990s, it is only in the recent decade that the analytical tools have reached a point where they can be used by practicing engineers. Major research has been performed in developing constitutive models such as the Mroz (1967) and Garud (1981) kinematic hardening models, to predict the elastic-plastic stresses and strains in a material under complex loading histories. Notch correction methods such as the ESED and multiaxial Neuber methods introduced by Singh et al.(1996) are then required in addition to the constitutive model to determine the stresses and strains in notched components. Multiaxial cycle counting 
procedures have been proposed along with a multitude of fatigue damage criteria to appropriately determine the fatigue life of variable amplitude load histories. Many comparative studies and reviews have been carried out by researchers such as Li (2009), Wang \& Yao (2004), and Fatemi \& Shamsaei (2011). A textbook covering the major aspects of multiaxial fatigue was published by Socie \& Marquis (2000) and provides an indepth coverage of the different fatigue damage criteria.

The purpose of this thesis is to explore the applicability of many of the multiaxial fatigue analysis methods to the analysis of the landing gear fuse pin. The multiaxial fatigue analysis includes four major components: the stress-strain model, the notch correction method, the cycle counting procedure, and the fatigue damage criterion. The stress-strain model selected for this thesis is the model proposed by Jiang \& Sehitoglu (1996a) and simplified by Shamsaei (2010). Three notch correction methods - the incremental ESED method and the multiaxial Neuber's rule proposed by Singh et al.(1996), and the Unified method proposed by Ye et al. (2008)- were selected for testing along with two major multiaxial cycle counting procedures - the Wang-Brown procedure, and the BannantineSocie procedure. Finally, four fatigue damage criteria were selected from Socie \& Marquis (2000) to represent various classes of multiaxial damage criteria that have been proposed: the Kandil-Brown-Miller, Fatemi-Socie, Smith-Watson-Topper, and Liu Virtual Strain Energy criteria.

All four components of the multiaxial fatigue analysis were incorporated into a custom computer program that required an elastic stress history as input. The first step in this thesis was to develop this program and perform tests to verify the correct implementation of each component of the fatigue analysis. The verification tests first required the correlation of elastic-plastic stress and strain predictions under proportional and non-proportional loading with experimental results of smooth specimens from literature. Once the smooth specimen behaviour was verified, the implemented notch correction methods were then tested against experimental data from the literature. Finally, cycle counting procedures were tested using ASTM standard (2011) examples as a benchmark. 
With all of the components of the software verified against experimental results from the literature the fatigue analysis of three test fuse pins (identified as Pin A, B, and C throughout the thesis) provided by UTC Aerospace Systems - Landing Systems (UTASLS) were then performed. Thirteen different locations in each fuse pin were analyzed for fatigue damage using the different combinations of fatigue damage criterion, notch correction method, and cycle counting procedure to determine the effect of each of these elements of the fatigue analysis. The fatigue failure of one of the fuse pins was used as a test point for evaluating the performance of the multiaxial fatigue analyses.

\subsection{Thesis Outline}

The objective of this thesis is to determine if the multiaxial approach to fatigue analysis can provide for better fatigue life predictions of the landing gear fuse pins. The fatigue damages for a total of thirteen locations in three different fuse pin designs (i.e. Pin A, B, and C) are calculated for different combinations of fatigue damage criterion, notch correction method, and cycle counting procedure. The results of these fatigue damages are compared to the results using traditional uniaxial fatigue analysis approaches and to experimental results from the tests performed by UTAS-LS. Good correlation with test results were found for some combinations of the fatigue analysis and recommendations are made for further testing of the fuse pins.

Chapter 2 provides introduces fatigue analysis fundamentals for both uniaxial and multiaxial fatigue analysis methods and establishes the problem of the analysis of the landing gear fuse pins. Chapters 3, 4, and 5 present the implementation of the multiaxial stress-strain model, the notch correction methods, and the cycle counting procedures, respectively. In each of those chapters, the methods and results of the verification tests that are used to ensure proper implementation of each of the fatigue analysis procedures is presented as well. Finally, the fatigue damage results for the test fuse pins are presented and compared to experimental results in Chapter 6. Conclusions and recommendations for future work in investigating the use of multiaxial fatigue analysis methods in the design of fuse pins are presented in Chapter 7. 


\section{Chapter: Background and Literature Review}

This chapter presents the fundamental concepts involved in the fatigue analysis of materials, focusing mainly on the behaviour of metals as it pertains to the design of landing gear fuse pins. Methodologies are introduced in a uniaxial context before being followed by a discussion of the current multiaxial fatigue analysis procedures. The state of the art of landing gear fuse pin design and fatigue analysis will be presented in the final sections of this chapter with the purpose of illustrating the need for implementing multiaxial fatigue analysis methodologies.

\subsection{Fatigue Analysis Fundamentals}

Before discussing uniaxial and multiaxial fatigue analysis methodologies, it is important to provide a basic introduction to the key elements of a fatigue analysis. A fatigue analysis first begins with a characterization of the loads and the load history of a component. The magnitude of the loads will dictate the type of approaches to be used for the fatigue analysis. With the load history understood, the monotonic and cyclic material response to these loads will need to be determined using empirical models developed through uniaxial tension/compression tests. If a notch is present in the component being analyzed, the effect of stress concentrations will also need to be understood. Finally, the accumulation of fatigue damage will also have to be understood and modeled to then determine the service life of the component being analyzed. All of these aspects of fatigue analysis are introduced in the following sections.

\subsubsection{Approaches to Fatigue Analysis}

Lightweight design in the aircraft industry has rapidly grown to use the fail-safe design principle, where the capability of structures to operate while sustaining damage is considered. This calls for the use of fracture mechanics to analyze the integrity of parts with pre-existing cracks. Life predictions are then made based on the number of cycles that are required to propagate a pre-existing crack to a critical length. Many fracture 
mechanics-based fatigue theories have been established with significant practical applications and are covered well in standard textbooks such as Anderson (2005).

While the application of the fail-safe design principle allows engineers to achieve much more lightweight designs, a more aggressive demand for safety can make it impractical for many components such as aircraft landing gear to apply such a design approach. In these types of components, factors such as large variances in load environment, inspection difficulty, and lack of structural redundancy will require a safe-life design philosophy. Safe-life fatigue design will make use of so-called total-life approaches, which make use of test data that capture the cycles required to cause a crack to initiate in the material.

\section{Stress-Life and Strain-Life Fatigue Analysis}

Total-life approaches to fatigue are used conservatively to predict the initiation of a crack in a component, and thus signaling the "failure" of the part. The first total-life approach that was developed was stress-based (often referred to as stress-life fatigue) and is used for low amplitude cyclic stresses where deformation is expected to be predominantly elastic. The stress-based approach generally presumes unconstrained deformation and thus neglects any consideration of strain in its analysis. The stress-based approach uses S-N curves to relate stress amplitudes to the number of cycles to failure and uses mean stress correction methods such as Soderberg, Goodman, Gerber, and Morrow relationships (Bannantine, Comer, \& Handrock, 1990). While simplistic in its approach, stress-based fatigue analyses are limited to elastic deformation. When significant plastic deformation occurs, a strain-based approach (strain-life fatigue) becomes the more appropriate method for predicting fatigue lives. Strain-based approaches alternatively use strain-life curves which, similar to S-N curves, show the relationship between the total strain amplitude and the fatigue life for a given material. Mean stress effects are captured by means of corrective relationships analogous to those of the stress-life approach and notch effects are captured through the prediction of elastic-plastic deformation in response to stress concentrations based on rules such as Neuber's Rule (Suresh, 1998). Procedures for both stress-life and strain-life fatigue analyses are well-established in the context of uniaxial fatigue analyses 
and are presented in many fatigue analysis textbooks such as Fuchs (1980), Dowling (1993), and Suresh (1998).

Overall, the strain-based approach is much more involved than the stress-based approach in that there are numerous stages involved in its analysis. However, even in the absence of plastic deformation, the strain-based approach has been shown to better reflect the nature of fatigue failure in engineering components as it takes into consideration the constrained nature of the deformation in real assemblies. As illustrated by Figure 2-1, the strain-life approximation is also found to reduce to the stress-life approximation in the high-cycle regime. This approach is thus considered to be a comprehensive approach that can simply replace the stress-based approach in all situations (Dowling, Mechanical Behavior of Materials, 1993). Using this philosophy that the following sections will focus on elements of fatigue analysis that are pertinent to a strain-based approach to fatigue analysis. The fundamental elastic-plastic behaviour of materials under monotonic and cyclic loading will be discussed to provide a basis upon which constitutive relations for materials can be developed. The effects of loading sequence and notches on the fatigue life of metals will be discussed and the concept of fatigue damage in the context of crack initiation will be introduced. 


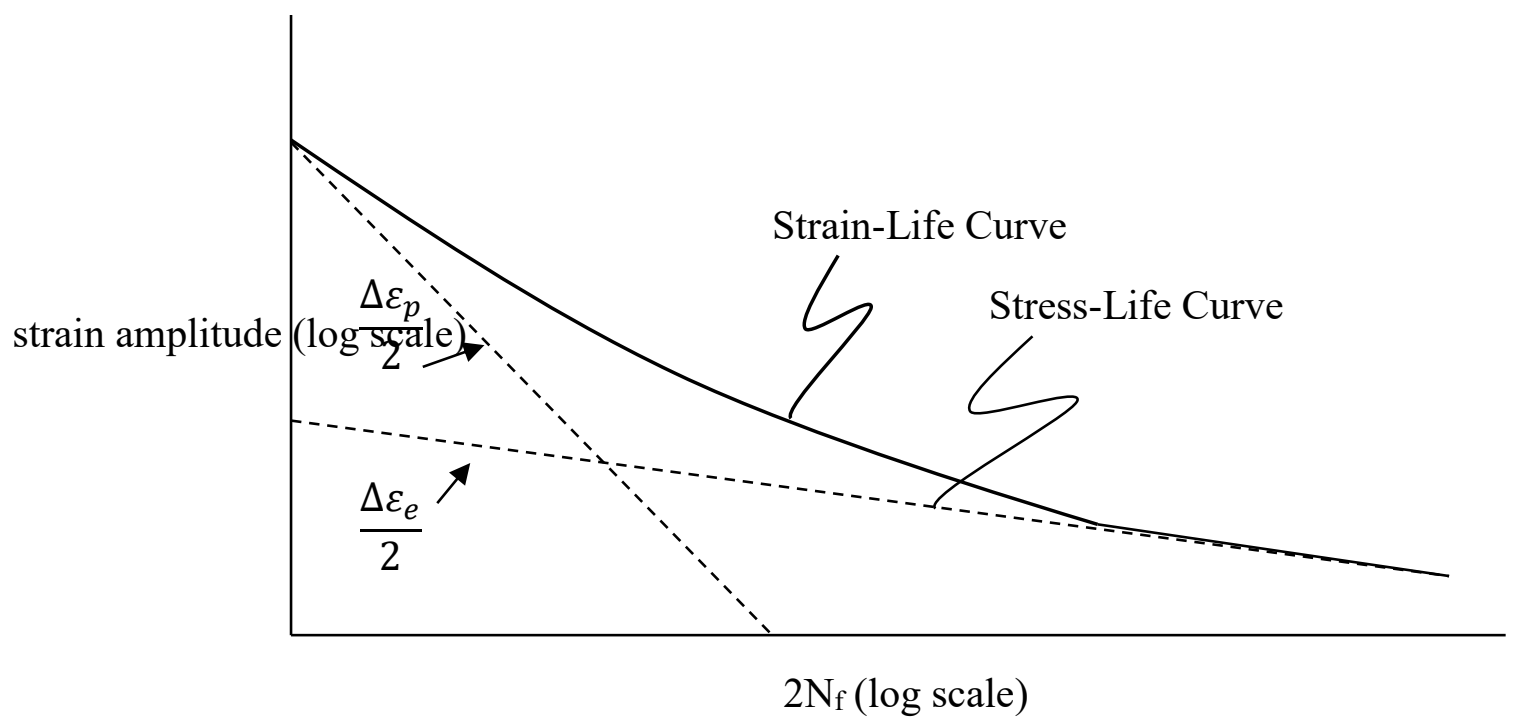

Figure 2-1: Schematic representation of stress-life and strain-life curve approximations

\subsubsection{Elastic-Plastic Behaviour of Materials under Monotonic Loading}

Under elastic loading conditions, stresses and strains are related linearly through Hooke's law as given by (2-1)for direct strains and (2-2) for shear strains, where $E$ is the Young's modulus and $v$ is Poisson's ratio and the components of the stresses and strains.

$$
\begin{gathered}
\varepsilon_{11}=\frac{1}{E}\left(\sigma_{11}-v\left(\sigma_{22}+\sigma_{33}\right)\right) \\
\varepsilon_{22}=\frac{1}{E}\left(\sigma_{22}-v\left(\sigma_{11}+\sigma_{33}\right)\right) \\
\varepsilon_{33}=\frac{1}{E}\left(\sigma_{33}-v\left(\sigma_{11}+\sigma_{22}\right)\right) \\
\frac{\gamma_{12}}{2}=\varepsilon_{12}=\frac{1+v}{E} \sigma_{12} \\
\frac{\gamma_{23}}{2}=\varepsilon_{23}=\frac{1+v}{E} \sigma_{12} \\
\frac{\gamma_{13}}{2}=\varepsilon_{13}=\frac{1+v}{E} \sigma_{12}
\end{gathered}
$$


Hooke's law can be expressed alternatively using a tensor notation as given by (2-3).

$$
\varepsilon_{i j}^{e}=\frac{1+v}{E} \sigma_{i j}-\frac{v}{E} \sigma_{k k} \delta_{i j}
$$

Here the Kronecker delta $\delta_{i j}$ is used and is defined by (2-4).

$$
\delta_{i j}= \begin{cases}1, & \text { for } i=j \\ 0, & \text { for } i \neq j\end{cases}
$$

In utilizing (2-5), the summation convention of indicial notation is used where on repeated indices a summation is implied as follows:

$$
\sigma_{k k}=\sigma_{11}+\sigma_{22}+\sigma_{33}
$$

Expressing Hooke's law in tensor form and introducing the indicial notation here will be useful in later sections for presenting more complex constitutive relations.

Once the stress in a material is large enough to cause plastic deformation, a non-linear relationship is required to relate the stress and the strain. Under uniaxial loading conditions, the Ramberg-Osgood relation expressed in (2-6) is the most commonly used formula to relate the stress to the total strain, which is the additive combination of the elastic strain given by Hooke's law and the plastic strain. This power-law relationship uses two parameters to capture the plastic strain component as it relates to stress: the monotonic strength coefficient $K$ and the strain hardening exponent $n$. These parameters are easily determined from the stress-strain curves generated from simple uniaxial tension tests.

$$
\varepsilon=\frac{\sigma}{E}+\left(\frac{\sigma}{K}\right)^{1 / n}
$$

Equations (2-2) and (2-6) provide the sufficient tools required for determining the complete stress-strain state of a material under monotonic loading conditions. 


\subsubsection{Elastic-Plastic Behaviour of Materials under Cyclic Loading}

For fatigue analyses the behaviour of materials under cyclic loading must be considered. Cyclic loading of a material introduces a number of new material behaviours that go beyond the simple plastic deformation seen in monotonic loading. The following discussion of these behaviours is a brief summary of the detailed account of the mechanisms and experimental observations provided by (Suresh, 1998).

After a certain amount of forward plastic deformation, it has been experimentally observed that the onset of yielding in a material will occur at a lower stress in the reversed loading direction than the original yield point in the forward direction. This effect of a shifting yield point, known as the Bauschinger effect, is illustrated Figure 2-2. This occurs due to the anisotropy in the material that is created due to the plastic deformation in the initial direction and is accounted for in advanced cyclic plasticity models by including a kinematic hardening rule that dictates how the plastic strain evolves with each load. The physical mechanism for this effect varies depending on the nature of the material; for example, the degree to which the Bauschinger effect will be observed in a given metal will vary for particle-hardened, age-hardened, and dispersion strengthened metal alloys.

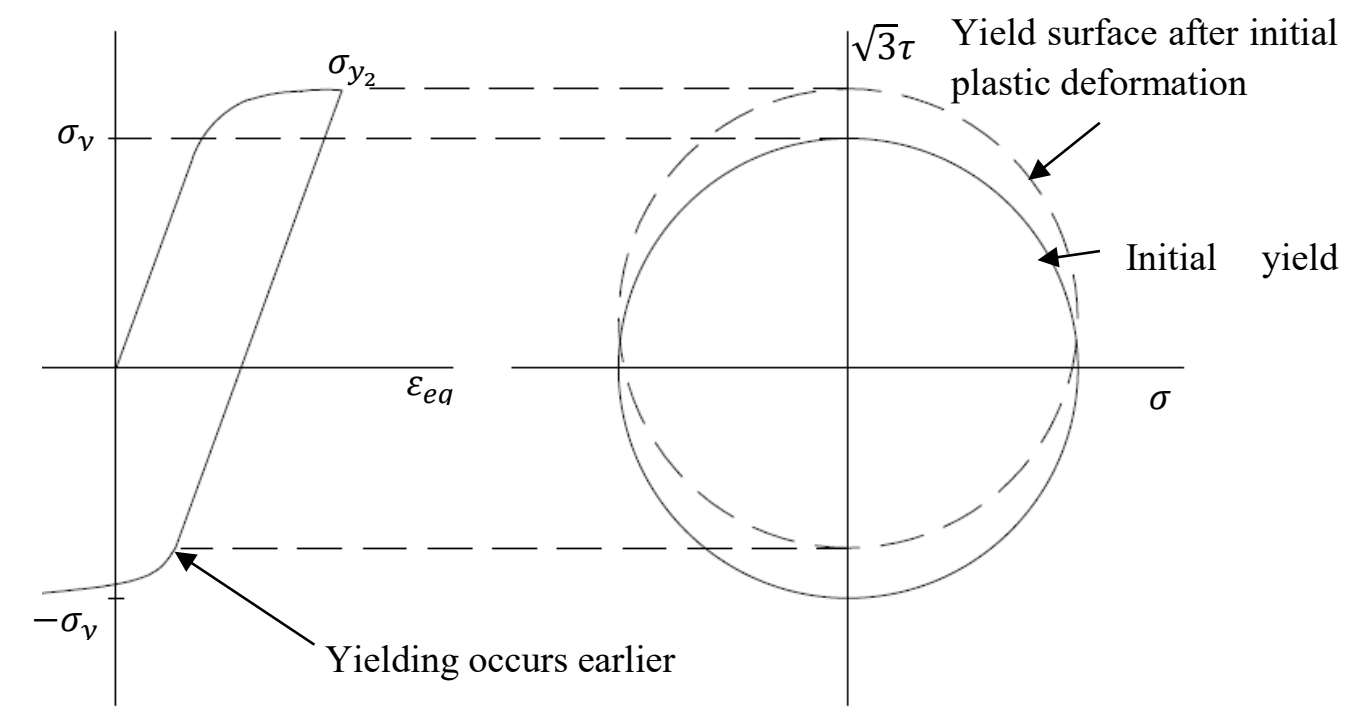

Figure 2-2: Schematic representation of kinematic hardening and the Bauschinger effect. 
As metals undergo cumulative plastic deformation through repeated cyclic loading, internal residual stresses are introduced into the metal matrix. In certain situations, a number of repeated cycles of loading can give rise to a state of equilibrium within the material where no net accumulation plastic deformation occurs and only recoverable elastic deformation is observed over a cycle. When the material reaches this saturated state it is said to have undergone elastic shakedown, or simply shakedown. This behaviour is often captured by including an isotropic hardening rule associated with the evolution of the plastic strain in a cyclic plasticity model. If a yield locus is considered to be the limits upon which a yield criterion is based, isotropic hardening is the progressive and uniform expansion of this yield locus with each plastic strain increment. This process is illustrated in Figure 2-3. Conversely, kinematic hardening is the translation of this yield locus that is associated with each plastic strain increment. After some degree of plastic straining, the yield locus will have increased to a size that a selected yield criterion no longer indicates plastic yielding and thus reflects the state of elastic shakedown.

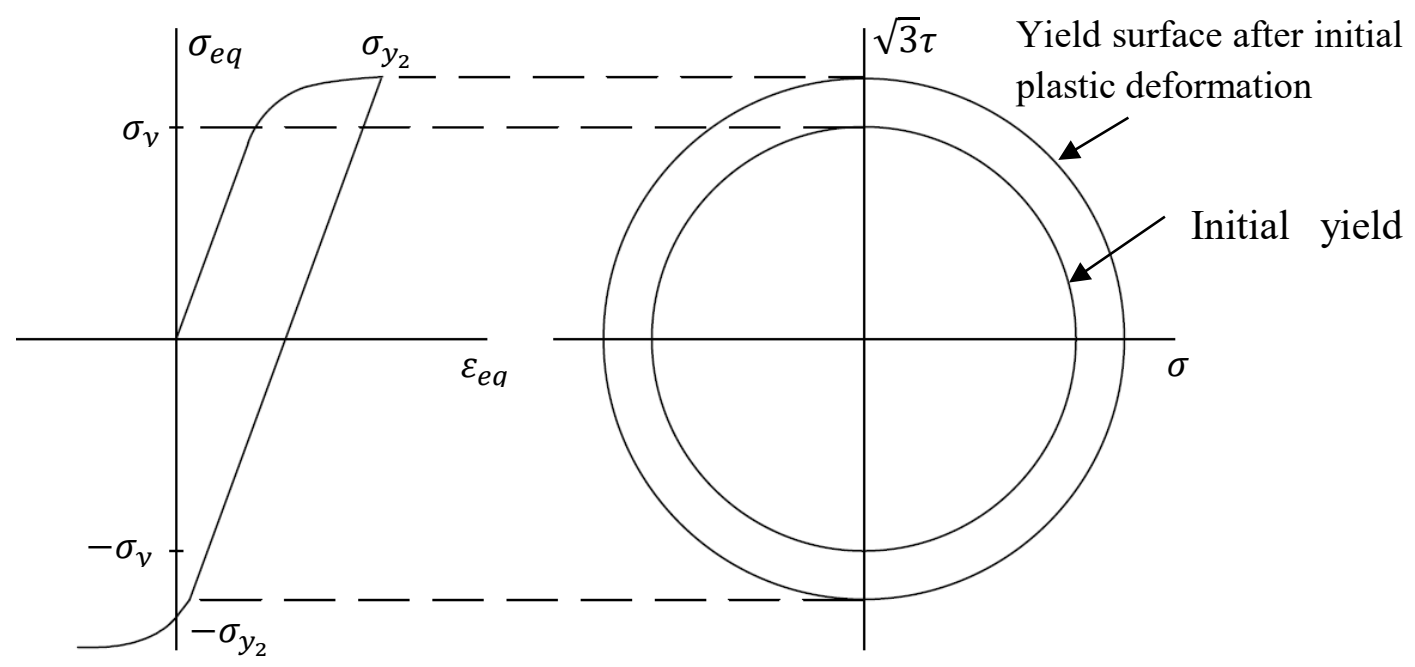

Figure 2-3: Schematic representation of isotropic hardening.

Analogous to the monotonic stress-strain curve, a cyclic stress-strain (CSS) curve is used to capture the Bauschinger effect and elastic shakedown of materials under cyclic loading. Equivalent to the standard uniaxial tensile test, constant amplitude strain-controlled tests 
are used to create CSS curves to observe the cyclic behaviour of ductile materials. During each cycle, a hysteresis loop is generated; however, each consecutive hysteresis loop will not be identical to its predecessor. As illustrated in Figure 2-4, the material can undergo a series of cyclic hardening or softening before reaching a state of saturated plastic deformation. The evolution of these hysteresis loops will depend on each individual material's response in terms of the Bauschinger effect and elastic shakedown.

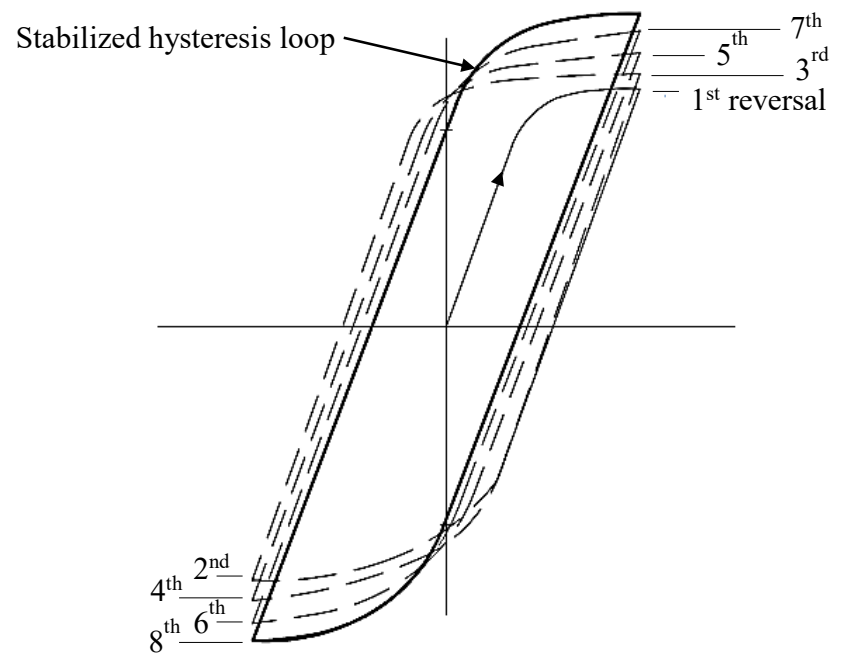

Figure 2-4: Example of stabilization of hysteresis loop under strain-controlled reversed loading.

Like the monotonic stress-strain curve, the CSS curve can also be modeled empirically based on a power-law assumption by an equation similar to the Ramberg-Osgood type equation:

$$
\frac{\Delta \varepsilon}{2}=\frac{\Delta \sigma}{2 E}+\left(\frac{\Delta \sigma}{2 K^{\prime}}\right)^{1 / n^{\prime}}
$$

where $\frac{\Delta \varepsilon}{2}$ is the total strain amplitude, $\frac{\Delta \sigma}{2}$ is the stress amplitude, $K^{\prime}$ is the cyclic strength coefficient, and $n^{\prime}$ is the cyclic strain hardening exponent. It is important to note that the use of the CSS curve to predict the strain amplitude based on the stress amplitude only reflects stabilized cyclic behaviour and thus captures the end product of the Bauschinger 
effect and elastic shakedown of materials. An additional complication when considering cyclic loads beyond the relationship of the magnitude of stresses and strains is the order in which they are applied to the material. The sequence of load application can significantly affect the overall stress-strain response and thus the fatigue life of a component. These load sequence effects are introduced and discussed in the following section.

\subsubsection{Load Sequence and its Effect on Fatigue Life}

In an ideal world, the exact loading scenario of a part would be known for the entire duration of its service. With the loads at any given point in time known, the stresses and strains in the part can be calculated to within the accuracy of the predicting model. In reality, engineers must predict the loads that the part being designed will encounter. The loading sequence, real or predicted, will vary. Many components will often see very consistent repeated loading with little variance. When a block of cycles contain the same stress or strain amplitude and mean stress or strain this sequence is referred to as constant amplitude loading. On the other hand, most components will see great variance in their loads. For example, an aircraft landing gear will see varying degrees of takeoff, taxiing, braking, and turning loads in a given day. These types of load histories, where the load amplitude varies from cycle to cycle, is referred to as variable amplitude loading.

For constant amplitude loading, fatigue analysis is relatively straightforward. For a particular cycle, the number of repetitions, stress and strain amplitude, and mean stress and strain are all known. In addition to the Bauschinger effect and elastic shakedown, the presence of a mean stress or strain can lead to ratchetting or mean stress relaxation, respectively. Ratchetting, also referred to as cyclic creep, is the effect of a steady progressive increase in plastic deformation per cycle in the forward or reversed loading direction due to a load sequence with constant cyclic stress amplitude and a tensile or compressive mean stress. Mean stress relaxation occurs in both cyclically hardening and softening materials when a sequence of repeated fixed cyclic strain amplitudes with a tensile mean strain. The effect of such a load sequence is a progressive reduction in mean stress eventually approaching a zero mean stress. 
Fatigue analyses become further complicated when considering variable amplitude loading, where sequence effects play a major role in determining the fatigue life of a material. Sequence effects observed in variable amplitude loading are rooted in the nature of crack initiation and propagation. The simple case of sequence effects is made by comparing the high-low and low-high load sequence examples. Starting with a number of high level loads a microscopic crack could be initiated. Following this high load sequence, a low level load sequence would then act to further grow the previously initiated crack at a faster rate than would otherwise have been expected. If the sequence of loads were reversed, a series of low level loads could be applied without significantly contributing to the number of cycles required for failure. Along with these basic sequence effects, block size effects, mean stress effects, and the effectiveness of cycle counting procedures were further investigated by (Dowling, 1972).

To simplify variable amplitude load sequences into blocks of constant amplitude loading for fatigue analyses, cycle counting procedures are used. These procedures attempt to capture the overall hysteresis loop that would be observed under a given set of cycles. Many procedures have been introduced over the decades. While (Dowling, 1972) notes that many proposed procedures have been clearly observed to produce unreasonable results, the range pair and rain flow counting procedures were shown to be the most reasonable and allows for accurate life predictions. Today these two methods are among the standard practice cycle counting procedures as recommended by the American Society for Testing Materials (ASTM International, 2011). These cycle counting procedures will be discussed in more detail in the context of a uniaxial fatigue analysis in 2.2.4.

\subsubsection{Effect of Notches}

Of significant interest in fatigue analysis is the effect of notches on the stress-strain response of the material. Geometrical discontinuities in materials will cause a local stress concentration. These geometrical discontinuities are colloquially referred to as notches, though this may refer to holes, shoulders, and true notches. This local stress concentration gives rise to potential local yielding which needs to be captured when predicting the stress 
and strain within a component. Under purely elastic loading conditions, a stress concentration factor $\left(K_{t}\right)$ can be defined by Eqn. (2-8) for a given geometry relating the peak stress at the notch to the nominal stress as illustrated in Figure 2-5:

$$
K_{t}=\frac{\sigma_{\max }}{\sigma_{\text {nom }}}
$$

Beyond simply inducing a localized maximum stress in the material, stress concentrations will affect the fatigue life of the component. In other words, for a given maximum stress amplitude the fatigue life of a component can be reduced significantly if that stress amplitude is in a location of a stress concentration compared to if that same stress amplitude was in a plain un-notched section. Stress-life fatigue approaches will make use of fatigue sensitivity factors to either modify the stresses or the fatigue life curves to account for the material response to a stress concentration (Suresh, 1998). On the other hand, strain-life fatigue approaches will make use of energy-based theories such as Neuber's rule in the uniaxial context and variations thereof in the multiaxial context. These methodologies will be discussed further in Sections 2.2.3 and 2.3.3.

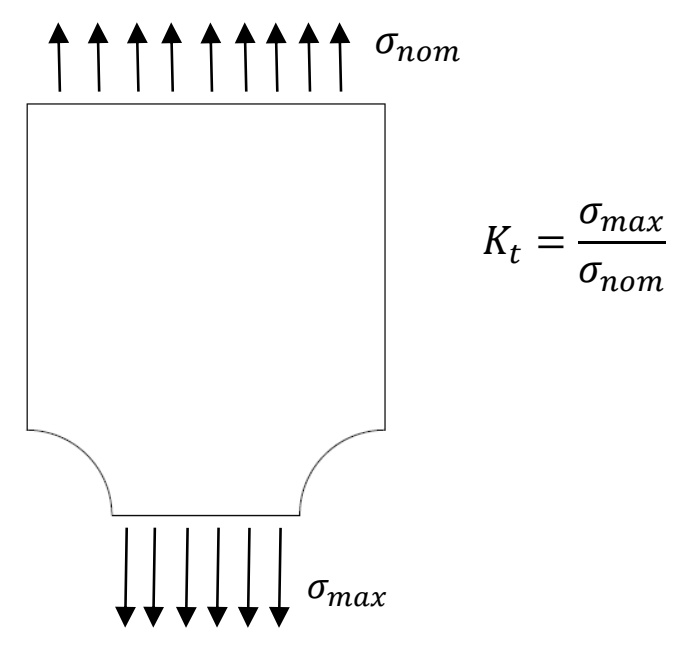

Figure 2-5: Schematic of notch stresses.

\subsubsection{Fatigue Damage}

Upon taking into account all of the material behaviours discussed previously, the final goal of a fatigue analysis is to determine the point of failure of the material. The fatigue failure 
of a material can be divided into two stages: crack initiation and crack propagation. During crack propagation, the measure of damage is simply the length of the crack. The mechanics involved in crack propagation and the definition of the critical crack length are all a part of the fracture mechanics field. The practice of incorporating crack propagation into design is referred to as damage-tolerant design and is covered in detail in many textbooks such as Anderson (2005). While most lightweight structures have now moved towards a damagetolerant design approach, many heavier components such as landing gear use the safelife design approach. This approach assumes no initial cracks and defines failure at the first sign of a detectable crack. The fatigue analysis of these components is thus limited to the analysis of materials within the crack initiation stage of failure.

In the absence of a physical measure of damage, researchers over the past 50 years have debated over what metric is appropriate for predicting the failure of a component. The first formally established rule, and the most well-known today, is the Palmgren-Miner linear damage rule, which can be expressed mathematically by (2-9).

$$
D=\sum_{i=1}^{m} \frac{n_{i}}{N_{f i}}=1
$$

where $n_{i}$ is the number of cycles corresponding to the $i$ th block of constant stress amplitude and $N_{f i}$ is the number of cycles to failure at that constant stress amplitude. The damage rule states that if the sum of the ratios of the allowable cycles $\left(n_{i}\right)$ and the allowable cycles $\left(N_{f i}\right)$ reaches a value of unity then failure will occur.

This linear damage rule has been popular in the industry due to its simplicity. However, it faces significant criticism due to a number of shortfalls: it neglects sequence effects of the load history; it neglects the effect of load-level; and it neglects the effect of loadinteraction. Over the decades, many new damage theories have been proposed that include other linear damage rules, non-linear damage rules, damage curve approaches, and various hybrid theories. An in-depth review of the recent damage theories is covered by Fatemi and Yang (1998). Since the purpose of this thesis is not to investigate the damage 
accumulation theories, all of the analyses in this thesis will simply make use of the Palmgren-Miner damage rule for fatigue life predictions.

\subsection{Uniaxial Fatigue Analysis}

While in most cases the stress state in a component is at the very least biaxial, there are many situations where a uniaxial approach is appropriate. The discussion presented in this section is a summary of the key concepts of uniaxial fatigue analysis as presented by Suresh (1998), Fuchs (1980), and Bannantine et al. (1990).

\subsubsection{Strain-Life Curves}

For a total life fatigue analysis, the strain-life approach uses strain-life curves that relate the total strain amplitude to the number of load reversals to failure as determined through experiments. The Basquin equation, originating from the stress-life approach to fatigue

analysis, relates the stress amplitude $\frac{\Delta \sigma}{2} \operatorname{logarithmically~to~the~number~of~reversals~to~failure~}$ $2 N_{f}$, where $\sigma_{f}^{\prime}$ and $b$ are fitting parameters referred to as the fatigue strength coefficient and the fatigue strength exponent, respectively:

$$
\frac{\Delta \sigma}{2}=\sigma_{f}^{\prime}\left(2 N_{f}\right)^{b}
$$

Applying Hooke's law to a uniaxial stress state, as shown below, Equation (2-10) is then modified to express the elastic strain amplitude $\frac{\Delta \varepsilon_{e}}{2}$ as a function of the number of reversals to failure:

$$
\begin{gathered}
\frac{\Delta \varepsilon_{e}}{2}=\frac{\Delta \sigma}{2 E} \\
\frac{\Delta \varepsilon_{e}}{2}=\frac{\sigma_{f}^{\prime}}{E}\left(2 N_{f}\right)^{b}
\end{gathered}
$$

The plastic strain amplitude can also be modeled logarithmically with respect to the number of load reversals to failure using the Coffin-Manson equation, where $\varepsilon_{f}^{\prime}$ and $c$ are additional 
fitting parameters referred to as the fatigue ductility coefficient and fatigue ductility exponent, respectively:

$$
\frac{\Delta \varepsilon_{p}}{2}=\varepsilon_{f}^{\prime}\left(2 N_{f}\right)^{c}
$$

Equations (2-11) and (2-12) are then combined to give Equation (2-13), the complete relationship between the total strain amplitude $\frac{\Delta \varepsilon}{2}$ and the number of load reversals to failure.

$$
\frac{\Delta \varepsilon}{2}=\frac{\sigma_{f}^{\prime}}{E}\left(2 N_{f}\right)^{b}+\varepsilon_{f}^{\prime}\left(2 N_{f}\right)^{c}
$$

This equation is referred to as the strain-life curve relation, or simply the strain-life relation. For pure torsional loads, a similar equation is given as follows:

$$
\frac{\Delta \gamma}{2}=\frac{\tau_{f}^{\prime}}{G}\left(2 N_{f}\right)^{b_{\gamma}}+\gamma_{f}^{\prime}\left(2 N_{f}\right)^{c_{\gamma}}
$$

In most cases, torsional strain-life data is not available. However, the following approximations based on the octahedral shear strain theory have been found to be appropriate:

$$
\begin{aligned}
\tau_{f}^{\prime} & \approx \frac{\sigma_{f}^{\prime}}{\sqrt{3}} \\
b_{\gamma} & \approx b \\
\gamma_{f}^{\prime} & \approx \sqrt{3} \varepsilon_{f}^{\prime} \\
c_{\gamma} & \approx c
\end{aligned}
$$

\subsubsection{Mean Stress Correction}

Two key methods that are used to account for the mean stress effect on fatigue life are the Morrow mean stress correction and the Smith-Watson-Topper mean stress correction methods. The Morrow mean stress correction method modifies the elastic strain term in the strain-life curve relation of Eqn. (2-13) to account for the mean stress $\sigma_{o}$ :

$$
\frac{\Delta \varepsilon}{2}=\frac{\sigma_{f}^{\prime}-\sigma_{o}}{E}\left(2 N_{f}\right)^{b}+\varepsilon_{f}^{\prime}\left(2 N_{f}\right)^{c}
$$


By assuming that the mean stress only affects the elastic component of the strain-life curve, predictions made with this correction effectively predict that the mean stress effects are more significant when there is less significant plastic strain than there is elastic. On the other hand, it has been shown that using the Morrow mean stress correction incorrectly predicts a dependence of the ratio of elastic and plastic strain on the mean stress.

The Smith-Watson-Topper mean stress correction method transforms the strain-life curve relation into an energy-based relationship by multiplying both sides of the equation by the maximum stress $\sigma_{\max }$ in the cycle. According to Eqn. (2-10), the maximum stress for completely reversed loading is given as

$$
\sigma_{\max }=\frac{\Delta \sigma}{2}=\sigma_{f}^{\prime}\left(2 N_{f}\right)^{b}
$$

Multiplying Eqn. (2-13) by Eqn. (2-17) then gives the following relationship:

$$
\sigma_{\max } \frac{\Delta \varepsilon}{2}=\frac{\sigma_{f}^{\prime 2}}{E}\left(2 N_{f}\right)^{2 b}+\sigma_{f}^{\prime} \varepsilon_{f}^{\prime}\left(2 N_{f}\right)^{b+c}
$$

For non-zero mean stress situations, the maximum stress is then evaluated as

$$
\sigma_{\max }=\frac{\Delta \sigma}{2}+\sigma_{o}
$$

The Smith-Watson-Topper method gains favour over the Morrow mean stress correction method as it correctly maintains an independence of the elastic-plastic strain ratio from the mean stress. However, the Smith-Watson-Topper mean stress correction falls short for cycles where the maximum stress is compressive and predicts zero fatigue damage for compressive stresses.

\subsubsection{Notch Correction: Neuber's Rule}

When the local stress in a material exceeds the yield strength, the stress and strain are no longer proportional. The nominal stress and strain, $S$ and $e$, then need to be related to the local stress and strain, $\sigma$ and $\varepsilon$, using two separate factors as given by Eqn. (2-20). 


$$
K_{\sigma}=\frac{\sigma}{S}, \quad K_{\epsilon}=\frac{\varepsilon}{e}
$$

Neuber's Rule, which was derived for longitudinally grooved shafts in torsion, states that the theoretical elastic stress concentration factor $K_{t}$ is the geometric mean of the stress and strain concentration and is given mathematically by Eqn. (2-21).

$$
K_{t}=\sqrt{K_{\sigma} K_{\epsilon}}
$$

Using Eqn. (2-20) and (2-21), the theoretical stress concentration factor can then be related to the nominal and local stresses and strains by the following equation:

$$
\sigma \varepsilon=K_{t}^{2} S e
$$

Eqn. (2-22) used in combination with the Ramberg-Osgood cyclic relationship given by Eqn. (2-7) provides a means of finding the local stress and strain in a notch by first calculating the linear stress and strain using the theoretical elastic stress concentration factor.

\subsubsection{Rainflow Cycle Counting}

Cycle counting procedures, as introduced in Section 2.1.4, are required as part of a fatigue analysis to simplify variable amplitude load sequences into blocks of constant amplitude loading. Once these blocks are identified, the stress and strain amplitude and the mean stress can be used with the strain-life curves to determine the number of reversals to failure. Among many cycle counting procedures, the range-pairing and rainflow counting procedures have been noted as the most reasonable. For notch-strain analyses, it has been noted that the rainflow counting procedure is the most advantageous method and thus this thesis will focus solely on this procedure.

Referring to Figure 2-6, the rainflow cycle counting procedure will walk through the load history while determining the relative magnitudes of the peaks and valleys. Reversals are 
considered to be every instance the load changes direction, and a cycle is the combination of two adjacent reversals. The rainflow procedure mostly focuses on identifying clear and independent reversals (half-cycles) but it also attempts to identify adjacent reversals to form a complete cycle. The detailed algorithm is for the rainflow cycle counting procedure is documented in ASTM E1049-85 (2011).

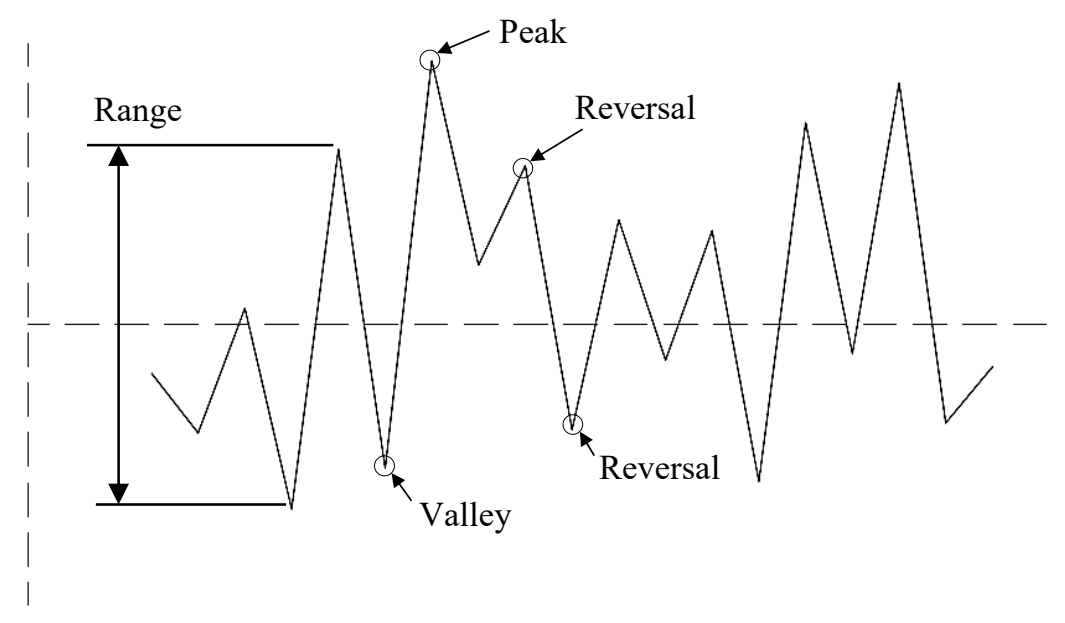

Figure 2-6: Illustration of cycle counting terminology.

\subsubsection{Residual Stresses at Notches}

When stress concentrations create local zones of plasticity in a component residual stresses are introduced to the material due to the surrounding elastic material. There are a number of empirical methods such that have been proposed to predict the residual stresses introduced by overloads at a notch. A graphical method was proposed by Potter (1971) that evaluated the cycle-by-cycle notch stress level to determine the resulting residual stresses. Methods similar to the method by Potter (1971) have been developed privately over the years by various companies and are often incorporated into in-house Neuber notch correction software. This thesis does not focus on the effects of residual stresses on the overall fatigue life of components. 


\subsubsection{Uniaxial Analysis of Multiaxial Stress States}

As mentioned before, most stress states in real components are multiaxial; that is, more than one component of stress will be present. Since most of the test data available are for uniaxial stress states either in tension/compression or torsion loading, the simplest approach to multiaxial stress states is to simplify the stress state to a uniaxial stress state. As inspired by its use as a yield criterion, the application of the octahedral shear strain energy theory, or Von Mises criterion, to simplify the multiaxial stress state has become the most popular. The von Mises stress is given by Eqn. (2-23).

$$
\sigma_{v m}=\frac{1}{\sqrt{2}} \sqrt{\left(\sigma_{x}-\sigma_{y}\right)^{2}+\left(\sigma_{y}-\sigma_{z}\right)^{2}+\left(\sigma_{x}-\sigma_{z}\right)^{2}+6\left(\tau_{x y}^{2}+\tau_{y z}^{2}+\tau_{x z}^{2}\right)}
$$

When simplifying the three-dimensional stress state to a von Mises stress using Eqn. (2-23), it is clear that only positive values will be obtained. In order to reflect reversals in load, a signed von Mises convention can be adopted. For example, if the loading is dominated by the shear stress component $\tau_{x y}$ the sign of the von Mises stress would be designated as being the same as the sign of $\tau_{x y}$. In a uniaxial fatigue analysis, this simplification of the multiaxial stress history to a signed von Mises stress history would be performed at the start of the analysis.

With a simplified signed von Mises stress history, the Neuber's rule along with the material stress-strain curve can then be used to determine the local stresses and strains which are then cycle counted using the rainflow cycle counting procedure. Once the cycles have been counted, fatigue lives are determined using the strain-life relation with or without the mean stress correction. This methodology is a major simplification of the mechanisms involved in fatigue failure.

In reality, microscopic fatigue cracks are initiated and propagated by the individual components of stress. Depending on the relative magnitudes of the stress components and the nature of failure of the material itself, fatigue life can be over-predicted or underpredicted if a uniaxial simplification is employed. The next section introduces the concepts 
involved in a complete multiaxial fatigue analysis, which removes the barriers involved in a uniaxial approach.

\subsection{Multiaxial Fatigue Analysis}

Multiaxial fatigue analyses will follow the same basic structure of a uniaxial fatigue analysis. Fatigue criteria are defined in place of the strain-life curve relations to relate the combination of stresses and strains in various directions to the number of reversals to failure that is found experimentally. To determine the stresses and strains for input into the fatigue criteria, a constitutive model is required to simulate the kinematic and isotropic hardening of the material. Cycle counting procedures, similar to the uniaxial versions, are employed to reduce the variable amplitude loading into blocks of constant amplitude loading for fatigue life evaluation. Finally, the fatigue damage can be calculated using rules such as the Palmgren-Miner linear damage rule. All of these aspects are explored in detail in this section.

\subsubsection{Multiaxial Fatigue Criteria}

\section{Equivalent Stress and Strain Model (Octahedral Shear Stress and Strain Theory)}

The simplest method for handling multiaxial fatigue problems is to use an equivalent stress or strain approach. These approaches are developed from stress-based static yield theories or strain-based theories. The approach presented here is similar to the uniaxial simplification presented in Section 2.2.6, however this procedure assumes that the threedimensional stress state that has been determined is already the local stress state; that is, the effects of the notch and strain hardening have already been accounted for.

An equivalent stress range can be defined based on the octahedral shear stress theory, as given by Equation (2-24).

$$
\Delta \sigma_{e q}=\frac{1}{\sqrt{2}} \sqrt{\left(\Delta \sigma_{x}-\Delta \sigma_{y}\right)^{2}+\left(\Delta \sigma_{y}-\Delta \sigma_{z}\right)^{2}+\left(\Delta \sigma_{x}-\Delta \sigma_{z}\right)^{2}+6\left(\Delta \tau_{x y}^{2}+\Delta \tau_{y z}^{2}+\Delta \tau_{x z}^{2}\right)}
$$


An effective value of mean stress $\bar{\sigma}_{m}$ may be defined in terms of the mean values of the principal stress, $\sigma_{i m}(i=1,2,3)$ as given by Equation $(2-25)$.

$$
\bar{\sigma}_{m}=\sigma_{1 m}+\sigma_{2 m}+\sigma_{3 m}
$$

Equations (2-24) and (2-25) can be used in a stress-life based approach to determine the fatigue life of a component subjected to multiaxial loading.

For strain-life fatigue analyses, an equivalent strain range can be calculated based on the octahedral shear strain theory.

$$
\Delta \varepsilon_{e q}=\frac{1}{\sqrt{2}(1+v)} \sqrt{\left(\Delta \varepsilon_{x}-\Delta \varepsilon_{y}\right)^{2}+\left(\Delta \varepsilon_{y}-\Delta \varepsilon_{z}\right)^{2}+\left(\Delta \varepsilon_{x}-\Delta \varepsilon_{z}\right)^{2}+\frac{3}{2}\left(\Delta \gamma_{x y}^{2}+\Delta \gamma_{y z}^{2}+\Delta \gamma_{x z}^{2}\right)}
$$

To include the effects of mean stress in the strain-life approach, the model proposed by Smith, Watson \& Topper can be used in a similar manner to Equation (2-18). In this model the maximum stress is noted to be $\bar{\sigma}_{\max }=\bar{\sigma}_{m}+\frac{\Delta \sigma_{e q}}{2}$, where the equivalent mean stress and stress range are calculated using Equations (2-24) and (2-25). The strain-life relation of Eqn. (2-13) can be modified to take the following form:

$$
\bar{\sigma}_{\max } \frac{\Delta \varepsilon_{e q}}{2}=\frac{\sigma_{f}^{\prime}}{E}\left(2 N_{f}\right)^{b}+\varepsilon_{f}^{\prime}\left(2 N_{f}\right)^{c}
$$

Although the equivalent stress/strain approaches are computationally the simplest methods to implement, they have significant limitations in their ability to predict the fatigue behaviour of materials. Yokobori et al. (1965) found that the octahedral shear strain range cannot distinguish between pure tension and pure torsion cases. This was proven by showing that for the same octahedral shear strain range, pure torsional loading will be less damaging than a pure tension case.

Another limitation in the use of equivalent stress/strain approaches is in the abstract nature of the calculations. These approaches cannot identify the failure mode or the plane upon which failure is expected to occur since these methods are essentially calculated average stresses and strains in a volume of material. 
In search of a more realistic and observation-based methodology for predicting fatigue failure of components, researchers eventually developed a number of critical plane models. These models are based on the observation that crack growth, depending on the stress and strain state, environment and material, will occur along either shear planes or tensile planes. Critical planes can be identified as those with the largest range of a particular stress component or strain component. Once the critical plane is identified, the fatigue life is evaluated using a formula similar to the strain-life relation of Eqn. (2-13). While there are both stress-based and strain-based models, this section will focus on the strain-based models for the reasons discussed in Section 2.1.1. The following discussion is a summary of that which is presented by Socie and Marquis (2000).

\section{Kandil-Brown-Miller Model}

The Kandil-Brown-Miller (KBM) model assumes that failure will occur on the plane of maximum shear strain range $\Delta \gamma_{\max }$. The final proposed strain-life relation is given by Eqn. (2-28):

$$
\frac{\Delta \gamma_{\max }}{2}+S \Delta \varepsilon_{n}=A \frac{\sigma_{f}^{\prime}-2 \sigma_{n, \text { mean }}}{E}\left(2 N_{f}\right)^{b}+B \varepsilon_{f}^{\prime}\left(2 N_{f}\right)^{c}
$$

where $\quad A=\left(1+v_{e}\right)+\left(1-v_{e}\right) S$

$$
B=\left(1+v_{p}\right)+\left(1-v_{p}\right) S
$$

The material parameter $S$ is determined based on the tension and torsion strain-life equations (2-13) and (2-14):

$$
S=\frac{\frac{\tau_{f}^{\prime}}{G}\left(2 N_{f}\right)^{b_{\gamma}}+\gamma_{f}^{\prime}\left(2 N_{f}\right)^{c_{\gamma}}-\left(1+v_{e}\right) \frac{\sigma_{f}^{\prime}}{E}\left(2 N_{f}\right)^{b}+\left(1+v_{p}\right) \varepsilon_{f}^{\prime}\left(2 N_{f}\right)^{c}}{\left(1-v_{e}\right) \frac{\sigma_{f}^{\prime}}{E}\left(2 N_{f}\right)^{b}+\left(1-v_{p}\right) \varepsilon_{f}^{\prime}\left(2 N_{f}\right)^{c}}
$$

The left-hand side of Eqn. (2-28) was originally defined by Kandil, Brown and Miller (1981) as an equivalent shear strain range. This equivalent shear strain range was then equated to the uniaxial strain-life equation modified both by the material parameter $S$ and the mean stress acting normal to the plane of maximum shear strain range. The result is a 
strain-life formulation that accounts for the normal strain and shear strain independently while employing a Morrow-type mean stress correction.

\section{Fatemi-Socie Model}

The Fatemi-Socie (FS) model is a modification to the KBM model in that it assumes, based on experimental observation, that the maximum normal stress $\sigma_{n, \max }$ should represent the crack-opening component of fatigue model in place of the normal strain. At the same time, since the maximum stress is included in the formulation a mean stress correction such as that seen in the KBM model is not required. The formulation proposed then comes in the following form:

$$
\frac{\Delta \gamma}{2}\left(1+k \frac{\sigma_{n, \max }}{\sigma_{y}}\right)=\frac{\tau_{f}^{\prime}}{G}\left(2 N_{f}\right)^{b_{\gamma}}+\gamma_{f}^{\prime}\left(2 N_{f}\right)^{c_{\gamma}}
$$

where the material constant $k$ is determined using the following relationship:

$$
k=\left(\frac{\frac{\tau_{f}^{\prime}}{G}\left(2 N_{f}\right)^{b_{\gamma}}+\gamma_{f}^{\prime}\left(2 N_{f}\right)^{c_{\gamma}}}{\left(1+v_{e}\right) \frac{\sigma_{f}^{\prime}}{E}\left(2 N_{f}\right)^{b}+\left(1+v_{p}\right) \gamma_{f}^{\prime}\left(2 N_{f}\right)^{c}}-1\right) \frac{\sigma_{y}}{\sigma_{n, \max }}
$$

Here it can be noted that the FS model equates the equivalent shear strain term (left-hand side of Eqn. (2-30)) to the torsional form of the strain-life relation.

Kim \& Park (1999) found that both the KBM and FS fatigue models provided acceptable life predictions for axial-torsion variable amplitude loading when used with the rainflow cycle counting on the shear strain history. Shamsaei et al. (2011) and Shamsaei \& Fatemi (2009)reported good correlation of fatigue life predictions for smooth tubular 1050 QT steel and 304L stainless steel specimens under various axial-torsion strain paths, both of which showed crack orientations to be around the maximum shear plane. Gates and Fatemi (2014) performed axial torsion tests on smooth and notched aluminum alloy 2024-T3 specimens and found that cracks initiated on planes of maximum shear stress for both smooth and notched specimens. For notched specimens it was found that the cracks in notched axial-torsion specimens of 2024-T3 aluminum would eventually change direction 
to grow on planes of maximum principal stress while cracks in the smooth specimens continued on the planes of maximum shear. For both smooth and notched specimens, Gates and Fatemi (2014) found that the use of the FS fatigue model correlated well with experimental failure mostly within a factor of 3 .

\section{Smith-Watson-Topper (SWT) Model}

In contrast to the KBM and FS models, the Smith-Watson-Topper (SWT) model is intended for use when a material is expected to fail along planes of maximum tension and compression. The SWT fatigue criterion equation follows the exact form of the SmithWatson-Topper mean stress corrected strain-life relation:

$$
\sigma_{n, \max } \frac{\Delta \varepsilon_{1}}{2}=\frac{\sigma_{f}^{\prime 2}}{E}\left(2 N_{f}\right)^{2 b}+\sigma_{f}^{\prime} \varepsilon_{f}^{\prime}\left(2 N_{f}\right)^{b+c}
$$

where $\frac{\Delta \varepsilon_{1}}{2}$ is the maximum principal strain range and $\sigma_{n, \max }$ is the maximum normal stress acting on the plane of maximum principal strain range.

Significant caution must be taken in applying methods such as the SWT model. As the fatigue criterion assumes for fatigue cracks to propagate under tensile action, significant errors can be observed when applied to materials that predominantly fail in shear modes. $\mathrm{Wu}$ et al. (2014) conducted tests on titanium alloy TC4 tubular specimens and found that the SWT fatigue model tended to predict significantly non-conservative fatigue lives for multiaxial and pure torsion load histories.

\section{Liu Virtual Strain Energy (VSE) Model}

The Liu model is a virtual strain energy (VSE) model that assumes that failure occurs on the plane having the maximum VSE quantity $\Delta W$. In multiaxial loading, $\Delta W$ is the sum of the axial and shear work acting on any given plane. The Liu model considers two planes of failure: (I) the plane on which the axial work is maximized, and (II) the plane on which the shear work is maximized. 
For plane I, the total VSE is calculated using Equation (2-33). Here $\Delta \tau \Delta \gamma$ is the shear work calculated on plane with maximum axial work.

$$
\begin{aligned}
& \Delta W_{I}=\left(\Delta \sigma_{n} \Delta \varepsilon_{n}\right)_{\max }+(\Delta \tau \Delta \gamma) \\
& =4 \sigma_{f}^{\prime} \varepsilon_{f}^{\prime}\left(2 N_{f}\right)^{b+c}+\frac{4 \sigma_{f}^{\prime}}{E}\left(2 N_{f}\right)^{2 b}
\end{aligned}
$$

For plane II, the total VSE is calculated using Equation (2-34). Here $\Delta \sigma \Delta \varepsilon$ is the axial work calculated on plane with maximum shear work.

$$
\begin{aligned}
& \Delta W_{I I}=\left(\Delta \sigma_{n} \Delta \varepsilon_{n)}+(\Delta \tau \Delta \gamma)_{\max }\right. \\
& =4 \tau_{f}^{\prime} \gamma_{f}^{\prime}\left(2 N_{f}\right)^{b_{\gamma}+c_{\gamma}}+\frac{4 \sigma_{f}^{\prime}}{G}\left(2 N_{f}\right)^{2 b_{\gamma}}
\end{aligned}
$$

For each VSE quantity, a corresponding fatigue life is calculated. The smaller value of fatigue life $N_{f}$ is then taken as the fatigue life of the component for that cycle. Advantages with using energy methods such as the Liu VSE method are attributed to the use of both the stress and strain terms in the fatigue damage formulation. A major downside to this method is the inability to consider the mean stress effects.

\subsubsection{Stress-Strain Analysis: Constitutive Modeling}

In uniaxial analyses, the formulations presented in Sections 2.1 .2 and 0 where a combination of stress-strain curves and Hooke's law were sufficient to have a complete understanding of the elastic-plastic stress-strain state in a material under monotonic and cyclic loading. While Hooke's law handles the elastic deformation in three dimensions, constitutive laws are required to determine how a material plastically deforms. Two categories of theories exist for defining plastic deformation: the deformation theories and the incremental plasticity theories. Whereas the deformation theories attempt to relate the total strain to the current stress state, incremental plasticity theories attempt to relate the increment of plastic deformation to the stress and strain increment for a given state of the material. Due to the stepped nature of incremental plasticity theories, these types of models have gained widespread use as computing power increased over the decades.

Any theory attempting to define such constitutive laws will contain the three basic elements of a theory of plasticity: 
1. A yield condition that defines the point at which plastic strains are obtained during an increment of load. Nearly all incremental plasticity models, as well as deformation theories, will use the von Mises criterion as defined by Equation (2-35)

$$
f=(\underline{S}-\underline{\alpha}):(\underline{S}-\underline{\alpha})-2 k^{2}=0
$$

where $\underline{S}$ is the deviatoric stress tensor, $\underline{\alpha}$ is the backstress tensor (identifying the center of the yield surface), and $k$ is the yield shear stress of the material. Note that $k=\sigma_{y} / \sqrt{3}$, where $\sigma_{y}$ is the yield strength in uniaxial tension. The yield surface is then defined as all points in the deviatoric stress space that satisfy Eqn. (2-35). Here the ":" operator denotes a tensor dot (inner) product. Note that the deviatoric stress tensor is defined by Eqn. (2-36) using tensor indicial notation.

$$
S_{i j}=\sigma_{i j}-\frac{1}{3} \sigma_{k k} \delta_{i j}
$$

2. A flow rule to compute the increment of plastic strain for an increment of load. The most common flow rule (the "normality flow rule") defines the plastic strain increment as being collinear with the exterior normal to the yield surface at the beginning of the increment. This is given mathematically as

$$
d \underline{\varepsilon}^{p}=\frac{1}{h}\langle d \underline{S}: \underline{n}\rangle \underline{n}
$$

where $d \underline{\varepsilon}^{p}$ is the plastic strain tensor increment, $d \underline{S}$ is the deviatoric stress increment, and $\underline{n}$ is the exterior unit normal to the yield surface at the loading point given by Equation (2-37):

$$
\underline{n}=\frac{\underline{S}-\underline{\alpha}}{|\underline{S}-\underline{\alpha}|}
$$


Note the McCaulay brackets used in Eqn. (2-37) to indicate that only deviatoric stress increments directed outwards of the yield surface will result in a non-zero plastic strain increment.

3. A hardening rule that defines how the yield condition changes under the increment of plastic strain. Hardening rules are either isotropic (yield surface increases in size with no translation, $\underline{\boldsymbol{\alpha}}=0$ ), kinematic (yield surface translates with no increase in size, $\mathrm{k}=$ constant) or mixed-mode (both isotropic and kinematic).

There are numerous incremental plasticity models that have been proposed in the last half century. Some models utilize the concept of field of work-hardening (plastic) moduli proposed by Mroz (1967) where the uniaxial stress-strain curve is approximated as being piece-wise linear, with each segment representing a constant plastic modulus of its own. Garud (1981) later proposed a modification to the Mroz kinematic hardening rule; a detailed comparison of these two methods is presented by Tipton and Bannantine (1993).

Currently, a popular model among several studies is the Armstrong-Frederick incremental plasticity model first proposed by Armstrong and Frederick (1966) and modified by Jiang and Sehitoglu (1996a), (1996b). The three key components of this plasticity model, henceforth referred to as the Jiang-Sehitoglu model, are the definitions of the backstress evolution, the plastic modulus function, and Tanaka's non-proportionality parameter.

\section{Backstress Evolution}

Since the backstress tensor identifies the centre of the yield surface in the deviatoric stress space, rules governing the evolution of the backstress tensor are kinematic hardening rules. In defining the hardening rule for the Jiang-Sehitoglu model, the total backstress is considered to be divided into $M$ components as follows:

$$
\underline{\alpha}=\sum_{i=1}^{M} \underline{\alpha}^{i}
$$

Using a series expansion of the backstress $\underline{\alpha}$ as given by (2-40) allows for the use of a nonlinear hardening rule to describe the evolution of the backstress. The Jiang-Sehitoglu 
model then defines the evolution of each backstress component based on the magnitude of the plastic strain increment $d p$ using the following relation:

$$
d \underline{\alpha}^{i}=c^{i} r^{i}\left(\underline{n}-\left(\frac{\left|\underline{\alpha}^{i}\right|}{r^{i}}\right)^{\chi^{i}+1} L^{i}\right) d p
$$

where $c^{i}$ and $r^{i}$ are material constants that can be calculated from the uniaxial cyclic stress-

strain curve, $\chi^{i}$ is a ratchetting rate constant determined through stress-controlled cyclic tests, and $\underline{L}^{i}$ and $d p$ are defined as follows:

$$
\begin{aligned}
& \underline{L}^{i}=\frac{\underline{\alpha}^{i}}{\left|\underline{\alpha}^{i}\right|} \quad(i=1,2,3, \ldots, M) \\
& d p=\left|d \underline{\varepsilon}^{p}\right|
\end{aligned}
$$

\section{Plastic Modulus Function}

Following the decomposition of the backstress, the plastic modulus function is then defined as a series expansion containing kinematic hardening and isotropic hardening components.

$$
h=\sum_{i=1}^{M} c^{i} r^{i}\left[1-\left(\frac{\left|\underline{\alpha}^{i}\right|}{r^{i}}\right)^{\chi^{i}+1} \underline{L}^{i}: \underline{n}\right]+\sqrt{2} \frac{d k}{d p}
$$

The first term of Eqn. (2-41) represents the change in the plastic modulus as the backstress evolves while the second term represents the isotropic hardening that takes place. Since the plastic modulus is the proportionality constant between the plastic strain increment $d \underline{\varepsilon}^{p}$ and the deviatoric stress component normal to the yield surface $\langle d \underline{S}: \underline{n}\rangle \underline{n}$ as seen in Eqn. (2-37), the process of determining the plastic strain increment based on an input stress increment will be an iterative process. 
A significant difference between uniaxial and multiaxial stress analysis is the consideration of the proportionality of the stress components during loading. Proportional loading is defined as loading paths that result in no rotation of the principal planes, which generally means that all of the stress components increase proportionally. Non-proportional loading is conversely defined as loading paths that result in a rotation of the principal planes. As an example, a load history where an axial load is held constant while a torsional load cycles would be considered non-proportional loading.

Non-proportional loading introduces an added complication to the modeling of strain hardening as research has shown that, depending on the material, additional hardening or softening will be observed as the degree of non-proportional loading increases. Kanazawa et al. (1979) studied the effects of combined axial and torsional loading on 1\% Cr-Mo-V steel and developed a linear correlation between the maximum shear stress and a rotation factor. This rotation factor relied on the factor of non-proportionality defined by Eqn. $(2-42)$.

$$
\Phi=\frac{\bar{\sigma}_{O P}}{\bar{\sigma}_{I P}}-1
$$

where $\bar{\sigma}_{O P}$ is the $90^{\circ}$ out-of-phase equivalent stress amplitude and $\bar{\sigma}_{I P}$ is the in-phase equivalent stress amplitude. This is a material parameter that represents the sensitivity of the material to non-proportional hardening and is dependent on the $90^{\circ}$ out-of-phase CSS curve and the in-phase (uniaxial) CSS curve. As the value of $\Phi$ will increase with the strain level, the calculation of $\Phi$ is generally performed at the highest strain level.

Instead of using (2-42) to find the factor of non-proportionality based on a comparison of the in-phase and out-of-phase CSS curves, Shamsaei and Fatemi (2010) proposed that the following estimation can be used to determine $\Phi$ based on standard uniaxial monotonic and cyclic material properties: 


$$
\Phi=1.6\left(\frac{K}{K^{\prime}}\right)^{2}\left(\frac{\Delta \varepsilon}{2}\right)^{2\left(n-n^{\prime}\right)}-3.8\left(\frac{K}{K^{\prime}}\right)\left(\frac{\Delta \varepsilon}{2}\right)^{\left(n-n^{\prime}\right)}+2.2
$$

Gates and Fatemi (2014) highlighted the importance of considering non-proportional hardening when modeling multiaxial stress states by demonstrating experimentally how out-of-phase loading in both 2024-T3 aluminum and a structural steel resulted in lower fatigue lives than in-phase loading at the same equivalent stress in both smooth and notched specimens. In contrast, Wu et al. (2014) found that non-proportional loading in titanium alloy TC4 did not produce any significant on the fatigue damage in low-cycle analyses. These two examples highlight the need for the non-proportionality parameter $\Phi$ to characterize the sensitivity of different materials to non-proportional loading.

Tanaka (1994) introduced a scheme for tracking the degree of non-proportionality of the load history using a fourth-order tensor $\underline{\underline{C}}$ which is initialized with zero value:

$$
d C_{i j k l}=C_{c}\left(n_{i j} n_{k l}-C_{i j k l}\right) d p
$$

where $C_{c}$ is a material constant. The fourth-order tensor $\underline{\underline{C}}$ given by Eqn. (2-44) is then used to determine an associated nonproportionality parameter defined as follows:

$$
A=\sqrt{1-\frac{n_{p q} C_{\alpha \beta p q} C_{\alpha \beta r s} n_{r s}}{C_{i j k l} C_{i j k l}}}
$$

Shamsaei et al. (2010) implemented Tanaka's nonproportionality constant $A$ in the following evolution equations for the yield stress and plastic modulus parameter $r^{i}$ :

$$
\begin{aligned}
& d k=\beta\left(k_{o} e^{(\sqrt{2} \phi+1) A}-k\right) d p \\
& d r^{i}=\beta\left[r_{o}^{i} e^{(\sqrt{2} \phi+1) A}-r^{i}\right] d p
\end{aligned}
$$


where $\beta$ is a nonproportionality hardening rate constant and the subscript $o$ denotes the initial values. Where only steady state responses are concerned a value of $\beta=5$ is found to provide good estimates for a wide range of applications. Note that the form of Eqn. (2-46) presented here corresponds to that presented in Socie and Marquis (2000), which was confirmed by the author to be the correct form. In their study of 1050 QT steel and 304L stainless steel, Shamsaei et al. (2010) found good correlation between strains predicted using Tanaka's nonproportionality constant and experimental strains. An alternative method for accounting for the non-proportionality of load histories has been proposed by Meggiolaro and Pinho de Castro (2014) that includes the calculation of a moment of inertia which is calculated for a wire tracing the strain path. This method is argued to better account for the individual contributions of every segment of the strain path in determining a non-proportionality factor.

\subsubsection{Notch Correction Methods}

Singh et al. (1996) proposed two analytical models for calculating the local stress and strains at a notch based on an equivalent strain energy density (ESED) approach: an incremental ESED method and an incremental Neuber's method. Both proposed models use the same set of constitutive equations presented in the following section. Though initially proposed with the Prandtl-Reuss incremental plasticity model, the following relations are based on the normality flow rule as presented with the Jiang-Sehitoglu incremental plasticity model discussed in Section 2.3.2.

\section{Manipulation of Constitutive Relations for Notch Corrections}

The normality flow rule given by Eqn. (2-37) can be expressed in tensor indicial notation as follows:

$$
d \varepsilon_{i j}^{p}=\frac{1}{h} n_{i j} d S_{s t} n_{s t}
$$

The total strain increment $d \varepsilon_{i j}$ can then be expressed as the summation of Eqn. (2-3) and Eqn.(2-47): 


$$
d \varepsilon_{i j}=\frac{1+v}{E} d \sigma_{i j}-\frac{v}{E} d \sigma_{k k} \delta_{i j}+\frac{1}{h} n_{i j} d S_{s t} n_{s t}
$$

Based on the definition of the deviatoric stress given by Eqn. (2-36) the deviatoric stress increments can be replaced such that

$$
d \varepsilon_{i j}=\frac{1+v}{E} d \sigma_{i j}-\frac{v}{E} d \sigma_{k k} \delta_{i j}+\frac{1}{h} n_{i j}\left(d \sigma_{s t}-\frac{1}{3} d \sigma_{k k} \delta_{s t}\right) n_{s t}
$$

Expanding out and applying the summation convention for indicial notation then results in the following set of equations for the components of the total strain increment:

$$
\begin{aligned}
& d \varepsilon_{11}=\left(\frac{1}{E}+n_{11} K_{1}\right) d \sigma_{11}+\left(n_{11} K_{2}-\frac{v}{E}\right) d \sigma_{22}+\left(n_{11} K_{3}-\frac{v}{E}\right) d \sigma_{33}+n_{11} K_{4} d \sigma_{12}+n_{11} K_{5} d \sigma_{23}+n_{11} K_{6} d \sigma_{13} \\
& d \varepsilon_{22}=\left(n_{22} K_{1}-\frac{v}{E}\right) d \sigma_{11}+\left(\frac{1}{E}+n_{22} K_{2}\right) d \sigma_{22}+\left(n_{22} K_{3}-\frac{v}{E}\right) d \sigma_{33}+n_{22} K_{4} d \sigma_{12}+n_{22} K_{5} d \sigma_{23}+n_{22} K_{6} d \sigma_{13} \\
& d \varepsilon_{33}=\left(n_{33}-\frac{v}{E}\right) K_{1} d \sigma_{11}+\left(n_{33} K_{2}-\frac{v}{E}\right) d \sigma_{22}+\left(\frac{1}{E}+n_{33} K_{3}\right) d \sigma_{33}+n_{33} K_{4} d \sigma_{12}+n_{33} K_{5} d \sigma_{23}+n_{33} K_{6} d \sigma_{13} \\
& d \varepsilon_{12}= n_{12} K_{1} d \sigma_{11}+n_{12} K_{2} d \sigma_{22}+n_{12} K_{3} d \sigma_{33}+\left(\frac{1+v}{E}+n_{12} K_{4}\right) d \sigma_{12}+n_{12} K_{5} d \sigma_{23}+n_{12} K_{6} d \sigma_{13} \\
& d \varepsilon_{23}= n_{23} K_{1} d \sigma_{11}+n_{23} K_{2} d \sigma_{22}+n_{23} K_{3} d \sigma_{33}+n_{23} K_{4} d \sigma_{12}+\left(\frac{1+v}{E}+n_{23} K_{5}\right) d \sigma_{23}+n_{23} K_{6} d \sigma_{13} \\
& d \varepsilon_{13}=n_{13} K_{1} d \sigma_{11}+n_{13} K_{2} d \sigma_{22}+n_{13} K_{3} d \sigma_{33}+n_{13} K_{4} d \sigma_{12}+n_{13} K_{5} d \sigma_{23}+\left(\frac{1+v}{E}+n_{13} K_{6}\right) d \sigma_{13}
\end{aligned}
$$

where

$$
\begin{aligned}
& K_{1}=\frac{1}{3 h}\left(2 n_{11}-\left(n_{22}+n_{33}\right)\right), K_{2}=\frac{1}{3 h}\left(2 n_{22}-\left(n_{11}+n_{33}\right)\right), \quad K_{3}=\frac{1}{3 h}\left(2 n_{33}-\left(n_{11}+n_{22}\right)\right) \\
& K_{4}=\frac{2 n_{12}}{h}, K_{5}=\frac{2 n_{23}}{h}, K_{6}=\frac{2 n_{13}}{h}
\end{aligned}
$$

Equation (2-50) makes up six of the twelve equations required to solve for all of the local stress and strain components at a notch. The remaining six equations can be either determined based on the incremental ESED method or the multiaxial Neuber's rule presented in the following sections. 


\section{Incremental ESED Method}

The ESED approach was used by Ince et al. (2014) to analyze the stress and strain of a SAE 1070 steel axial-torsion specimen under non-proportional loading. Using the cyclic plasticity model proposed by Garud (1981), it was shown that the incremental ESED method was able to produce reasonably accurate predictions of the local stresses and strains

at the notch using linear-elastic FE stress histories. The increment in strain energy in the body per unit volume due to an increment of applied load is given by Equation (2-51).

$$
d W^{a}=\sigma_{i j}^{a} d \varepsilon_{i j}^{a}
$$

where the superscript $a$ denotes the actual strain energy density increment, stress component, or strain increment. It is further proposed by Singh (1996) that the increment in the strain energy density at the notch tip in an elastic-plastic body can be approximated by that obtained if the body was to hypothetically remain elastic throughout the loading history; that is, the increment in strain energy density is the same. This is expressed by Equation (2-52).

$$
\mathrm{d} W^{e}=\mathrm{d} W^{E} \quad \text { or } \quad \sigma_{i j}^{e} \mathrm{~d} \varepsilon_{i j}^{e}=\sigma_{i j}^{E} \mathrm{~d} \varepsilon_{i j}^{E}
$$

The superscripts $e$ and $E$ denote the notch variables determined using linear elastic theory and/or FEA and the real notch variables as determined by the incremental ESED approach, respectively. As reference, Equation (2-52) would yield the following expression:

$$
\begin{aligned}
& \sigma_{11}^{e} \mathrm{~d} \varepsilon_{11}^{e}+\sigma_{22}^{e} \mathrm{~d} \varepsilon_{22}^{e}+\sigma_{33}^{e} \mathrm{~d} \varepsilon_{33}^{e}+2 \sigma_{12}^{e} \mathrm{~d} \varepsilon_{12}^{e}+2 \sigma_{23}^{e} \mathrm{~d} \varepsilon_{23}^{e}+2 \sigma_{13}^{e} \mathrm{~d} \varepsilon_{13}^{e} \\
& =\sigma_{11}^{E} \mathrm{~d} \varepsilon_{11}^{E}+\sigma_{22}^{E} \mathrm{~d} \varepsilon_{22}^{E}+\sigma_{33}^{E} \mathrm{~d} \varepsilon_{33}^{E}+2 \sigma_{12}^{E} \mathrm{~d} \varepsilon_{12}^{E}+2 \sigma_{23}^{E} \mathrm{~d} \varepsilon_{23}^{E}+2 \sigma_{13}^{E} \mathrm{~d} \varepsilon_{13}^{E}
\end{aligned}
$$

This equation thus provides one additional equation for the system of linear equations.

Assuming that the contribution of each elastic-plastic stress and strain component to the increment in strain energy density at the notch tip is the same as the contribution of each 
stress-strain component to the increment in strain energy density at the notch tip obtained from a linear elastic analysis, the following relationship holds true:

$$
\sigma_{\alpha \beta}^{e} \mathrm{~d} \varepsilon_{\alpha \beta}^{e}=\sigma_{\alpha \beta}^{E} \mathrm{~d} \varepsilon_{\alpha \beta}^{E}
$$

Note that in this case although $\alpha=\beta=1,2,3$ they are not used as Einstein indices and are not subject to an implied summation. Equation (2-53) then yields the following relationships:

$$
\begin{aligned}
& \sigma_{11}^{e} \mathrm{~d} \varepsilon_{11}^{e}=\sigma_{11}^{E} \mathrm{~d} \varepsilon_{11}^{E}, \quad \sigma_{22}^{e} \mathrm{~d} \varepsilon_{22}^{e}=\sigma_{22}^{E} \mathrm{~d} \varepsilon_{22}^{E}, \quad \sigma_{33}^{e} \mathrm{~d} \varepsilon_{33}^{e}= \\
& \sigma_{33}^{E} \mathrm{~d} \varepsilon_{33}^{E} \\
& \sigma_{12}^{e} \mathrm{~d} \varepsilon_{12}^{e}=\sigma_{12}^{E} \mathrm{~d} \varepsilon_{12}^{E}, \quad \sigma_{23}^{e} \mathrm{~d} \varepsilon_{23}^{e}=\sigma_{23}^{E} \mathrm{~d} \varepsilon_{23}^{E}, \quad \sigma_{13}^{e} \mathrm{~d} \varepsilon_{13}^{e}= \\
& \sigma_{13}^{E} \mathrm{~d} \varepsilon_{13}^{E}
\end{aligned}
$$

Five of the six equations in equation set (2-54) are linearly independent from Equation (2-52), thus completing the system of equations necessary to solve for the stress increment components $\left(d \sigma_{11}, d \sigma_{22}, d \sigma_{33}, d \sigma_{12}, d \sigma_{23}, d \sigma_{13}\right)$ and the elastic and plastic strain increment components $\left(d \varepsilon_{11}, d \varepsilon_{22}, d \varepsilon_{33}, d \varepsilon_{12}, d \varepsilon_{23}, d \varepsilon_{13}\right)$.

\section{Multiaxial Incremental Neuber's Method}

Rather than using the strain energy density as the basis of a new set of linear equations, Singh (1996) also proposed an approach that is based on the total strain energy density per unit volume $\Omega$, (sum of the strain energy density and the complementary strain energy density per unit volume), where the actual incremental change in total strain energy density per unit volume is given by

$$
\mathrm{d} \Omega^{a}=\sigma_{i j}^{a} \mathrm{~d} \varepsilon_{i j}^{a}+\varepsilon_{i j}^{a} \mathrm{~d} \sigma_{i j}^{a}
$$

As was the case with the incremental ESED approach, it was proposed that the increment in total strain energy density per unit volume is the same for an elastic and an elastic-plastic 
case, as expressed by Equation (2-56). The superscript $N$ denotes the real local notch variables as determined by the multiaxial incremental Neuber's method.

$$
\begin{gathered}
\Delta \Omega^{e}=\Delta \Omega^{N} \\
\text { or } \\
\sigma_{i j}^{e} \Delta \varepsilon_{i j}^{e}+\varepsilon_{i j}^{e} \Delta \sigma_{i j}^{e}=\sigma_{i j}^{N} \Delta \varepsilon_{i j}^{N}+\varepsilon_{i j}^{N} \Delta \sigma_{i j}^{N}
\end{gathered}
$$

Assuming that the contribution of each elastic-plastic stress and strain component to the increment in total strain energy density at the notch tip is the same as the contribution of each stress-strain component to the increment in total strain energy density at the notch tip obtained from a linear elastic analysis, the following relationship also holds true:

$$
\sigma_{\alpha \beta}^{e} \Delta \varepsilon_{\alpha \beta}^{e}+\varepsilon_{\alpha \beta}^{e} \Delta \sigma_{\alpha \beta}^{e}=\sigma_{\alpha \beta}^{N} \Delta \varepsilon_{\alpha \beta}^{N}+\varepsilon_{\alpha \beta}^{N} \Delta \sigma_{\alpha \beta}^{N}
$$

Analogous to the incremental ESED approach, the multiaxial incremental Neuber's method uses one equation produced by Equation (2-56) and five produced by Equation set $(2-57)$ to complete the system of linear equations. This system of linear equations is then solved simultaneously to find the stress increment components ( $d \sigma_{11}, d \sigma_{22}, d \sigma_{33}$, $\left.d \sigma_{12}, d \sigma_{23}, d \sigma_{13}\right)$ and the elastic and plastic strain increment components $\left(d \varepsilon_{11}, d \varepsilon_{22}, d \varepsilon_{33}\right.$, $\left.d \varepsilon_{12}, d \varepsilon_{23}, d \varepsilon_{13}\right)$.

Incremental Unified Method

Ye et al. (2008) proposed the use of an energy dissipation coefficient $\mathrm{C}_{\mathrm{q}}$ given by Eqn. (2-58). This energy dissipation coefficient is then incorporated into Equations (2-51) and (2-52) as shown in Eqn. (2-59) for the total ESED relation and in Eqn. (2-60) for the energy strain ratio relations.

$$
C_{q}=\frac{1-2 n^{\prime}}{1-n^{\prime}}
$$

where $n^{\prime}$ is the cyclic hardening exponent. 


$$
\begin{gathered}
\sigma_{i j}^{e} d \varepsilon_{i j}^{e}+\varepsilon_{i j}^{e} d \sigma_{i j}^{e}=\left(1+C_{q}\right) \sigma_{i j}^{U} d \varepsilon_{i j}^{U}+\left(1-C_{q}\right) \varepsilon_{i j}^{U} d \sigma_{i j}^{U} \\
\sigma_{\alpha \beta}^{e} d \varepsilon_{\alpha \beta}^{e}+\varepsilon_{\alpha \beta}^{e} d \sigma_{\alpha \beta}^{e}=\left(1+C_{q}\right) \sigma_{\alpha \beta}^{U} d \varepsilon_{\alpha \beta}^{U}+\left(1-C_{q}\right) \varepsilon_{\alpha \beta}^{U} d \sigma_{\alpha \beta}^{U}
\end{gathered}
$$

The predictions made by the incremental unified method are reported to produce better approximations than both the incremental ESED method and the multiaxial Neuber's rule, with each producing a lower and upper bound to experimental measurements, respectively (Ye, Hertel, \& Vormwald, 2008). Equations (2-59) and (2-60) approach the ESED formulations for $C_{q}=1$ and reduces to the Neuber's rule relations for $C_{q}=0$. The general algorithm for using the incremental ESED, multiaxial incremental Neuber's rule, and unified method is illustrated in Figure 2-7.

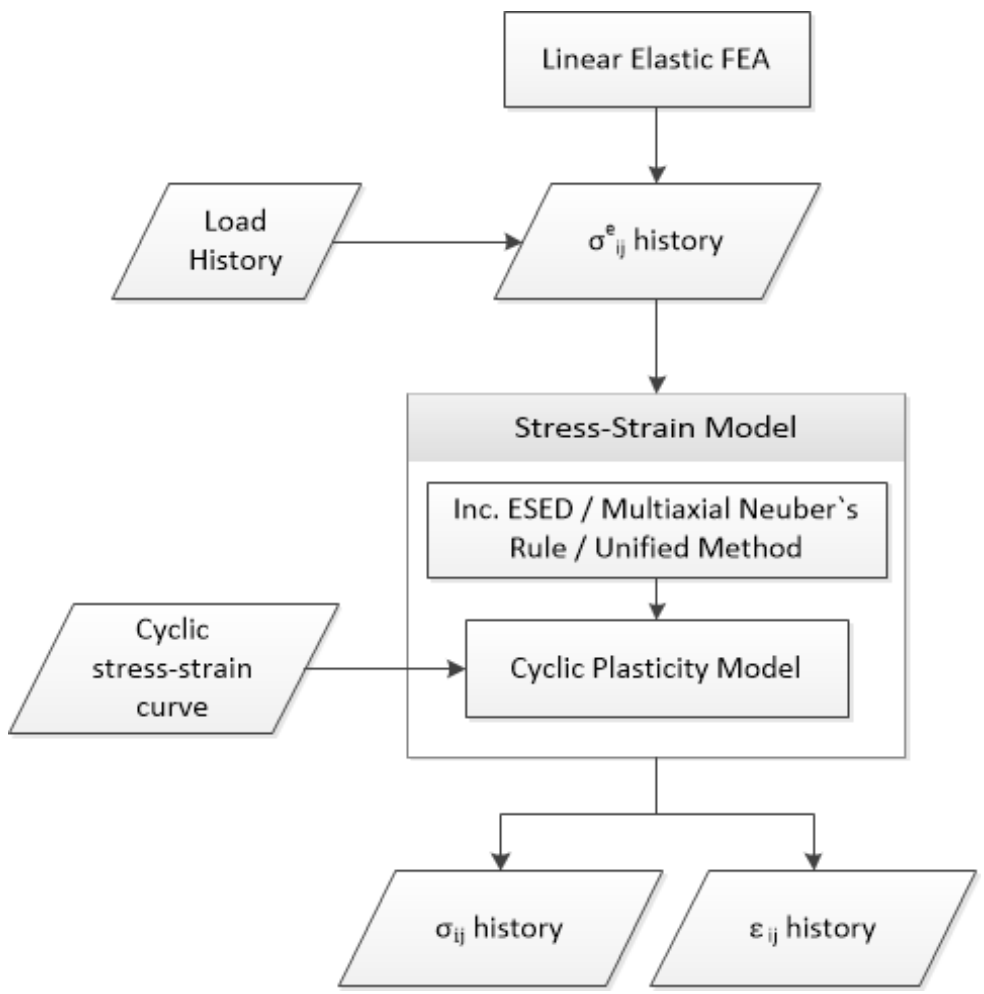

Figure 2-7: General procedure for implementing multiaxial notch correction methods. 


\subsubsection{Cycle Counting Procedures}

While the rainflow cycle counting procedure was developed for a uniaxial fatigue analysis, equivalent methods have been introduced to take into account multiaxial stress states either by incorporating the rainflow procedure differently or by taking inspiration from the rainflow procedure. Shamsaei et al. (2011) compared the Wang-Brown and BannantineSocie cycle counting procedures; noting that these are the only two methods that have been proposed that reflect the critical plane concept. In their evaluation of various loading histories for 1050 QT steel and 304 stainless steel, it was found that both cycle counting procedures produced satisfactory predictions when coupled with the FS fatigue criterion. The details involved in these two cycle counting procedures are presented in this section.

\section{Wang-Brown Cycle Counting Procedure}

The Wang-Brown cycle counting procedure is based in the use of the von Mises criterion. The procedure proposed by Wang \& Brown (1996) is summarized hereafter.

The cycle counting procedure assumes that the total strain history $\varepsilon_{i j}(t)$ is known. Rather than considering the history as a function of time, the remainder of this document will refer to each total strain tensor as $\left(\varepsilon_{i j}\right)_{k}$, where $k=1,2,3, \ldots n$ is the load number of the total strain tensor and $n$ is the total number of load cases.

Throughout the procedure, a relative strain history $\left(\varepsilon_{i j}\right)_{k}^{A}$ is defined as follows:

$$
\left(\varepsilon_{i j}\right)_{k}^{A}=\left(\varepsilon_{i j}\right)_{k}-\left(\varepsilon_{i j}\right)_{A}
$$

where $\left(\varepsilon_{i j}\right)_{A}$ is the reference strain tensor for the relative strain history. 
The basis for the cycle counting procedure will be the equivalent strain $\varepsilon^{e q}$ defined by:

$$
\begin{aligned}
\varepsilon^{e q} & =\frac{1}{\sqrt{2}\left(1+v^{\prime}\right)} \sqrt{\left(\varepsilon_{x}-\varepsilon_{y}\right)^{2}+\left(\varepsilon_{y}-\varepsilon_{z}\right)^{2}+\left(\varepsilon_{z}-\varepsilon_{x}\right)^{2}+\frac{3}{2}\left[\gamma_{x y}^{2}+\gamma_{y z}^{2}+\gamma_{z x}^{2}\right]} \\
& \left.=\frac{1}{\sqrt{2}\left(1+v^{\prime}\right)} \sqrt{2 \varepsilon_{x}^{2}\left(1+v^{\prime}\right)^{2}+\frac{3}{2} \gamma_{x y}^{2}} \quad \text { (simple axial }+ \text { torsion }\right)
\end{aligned}
$$

The following cycle counting procedure is then followed until every data point has been counted:

1. Arrange the original data file so that it starts with the first major turning point, i.e. the point with the peak value of equivalent strain. This is done by identifying the maximum turning point and moving all load cases occurring before this point to the end of the history. This load case then becomes the reference point (Point A) for the subsequent analysis.

2. Calculate the relative strain history with respect to the first data point $\left(\varepsilon_{i j}\right)_{A}$ of the strain history using Equation (2-61).

3. Identify the maximum peak (turning point) (Point B) of the relative strain history.

4. Identify fragment points, which are defined as any point at which the equivalent relative strain history falls prior to and after the maximum peak.

5. The load sequence from point $\mathrm{A}$ to point $\mathrm{B}$ is then identified as one reversal.

6. The data points from each fragment up to the point where the history resumes its upward progression to the maximum peak will be referred to as its corresponding fragmented history. For each fragment, repeat steps 2 through 5 with the corresponding fragment point as the reference point $\left(\varepsilon_{i j}\right)_{A}$.

Note that once the analysis begins for the sub-histories of each fragment, additional fragments will be found and the procedure will be repeated until all peaks are counted as reversals. Chapter 5 walks through an example presented by Wang \& Brown (1996). 


\section{Bannantine-Socie Cycle Counting Procedure}

The cycle counting procedure proposed by Bannantine and Socie (1989) is rooted in the philosophy of the critical plane approach to multiaxial fatigue analysis. The procedure is defined as follows:

1. Determine a critical plane either by experience or arbitrarily.

2. Evaluate the stress and strain history on the critical plane selected in Step 1.

3. Based on the nature of material, select the critical crack initiating or propagating strain component.

4. Carry out the rainflow cycle counting procedure as defined in ASTM E1048-85 on the strain component selected in Step 3.

5. Calculate the fatigue damage based on the counted cycles.

6. Repeat steps 1-5 for as many critical planes as is required to determine the plane with the highest fatigue damage.

\subsubsection{Residual Stresses}

Residual stresses are created when local yielding occurs at a notch. As was introduced in Section 2.2.5, residual stresses can be beneficial when compressive and damaging when tensile. To the author's knowledge, there is currently no analytical method for estimating the residual stresses that are formed at a notch after the unloading of a multiaxial stress state that is equivalent to the uniaxial approach. The only method to address this as the current research stands is to perform an elastic-plastic finite element analysis of the component in question and determine the residual stresses upon removal of a static load.

\subsection{Landing Gear Fuse Pin Design and Analysis: State of the Art}

The purpose of this thesis is to demonstrate the applicability of the multiaxial approach to fatigue analysis in improving the life prediction of landing gear fuse pins. Fuse pins are used in landing gear designs to provide a safe and controlled separation of the landing gear from the aircraft in the event of a crash. This controlled failure is achieved by the use of grooves circumferentially machined into the inner diameter of the fuse pin at the location 
of maximum shear load. These grooves introduce a stress concentration to an area in the fuse pin that is already highly stressed. In combination with the bearing load on the pin in the joint and the bending loads, the simple shear load in the fuse pin joint then creates a complex stress state at the fuse pin groove. The following sections discuss the basic design of fuse pins and the current state of the fatigue analysis of the fuse pin groove.

\subsubsection{Fuse Pin Stress State and Joint Design}

In general, fuse pins are used in three basic types of joints: a clevis joint with bushings, a cantilever joint with bushings, and a cantilever joint with a spherical bearing. Schematics of these three typical joints are presented in Figure 2-8, Figure 2-9, and Figure 2-10. Between the three joint assemblies, the degree of constraint at the joint will vary; the clevis joint has the highest level of constraint and the cantilever joint with spherical bearing has the lowest level of constraint. Regardless of the type of joint, the fuse pin groove will be subject to three basic components of stress: the shear stress, the longitudinal stress due to bending, and the hoop stress due to the bearing loads on the pin. This is illustrated in Figure 2-11. 


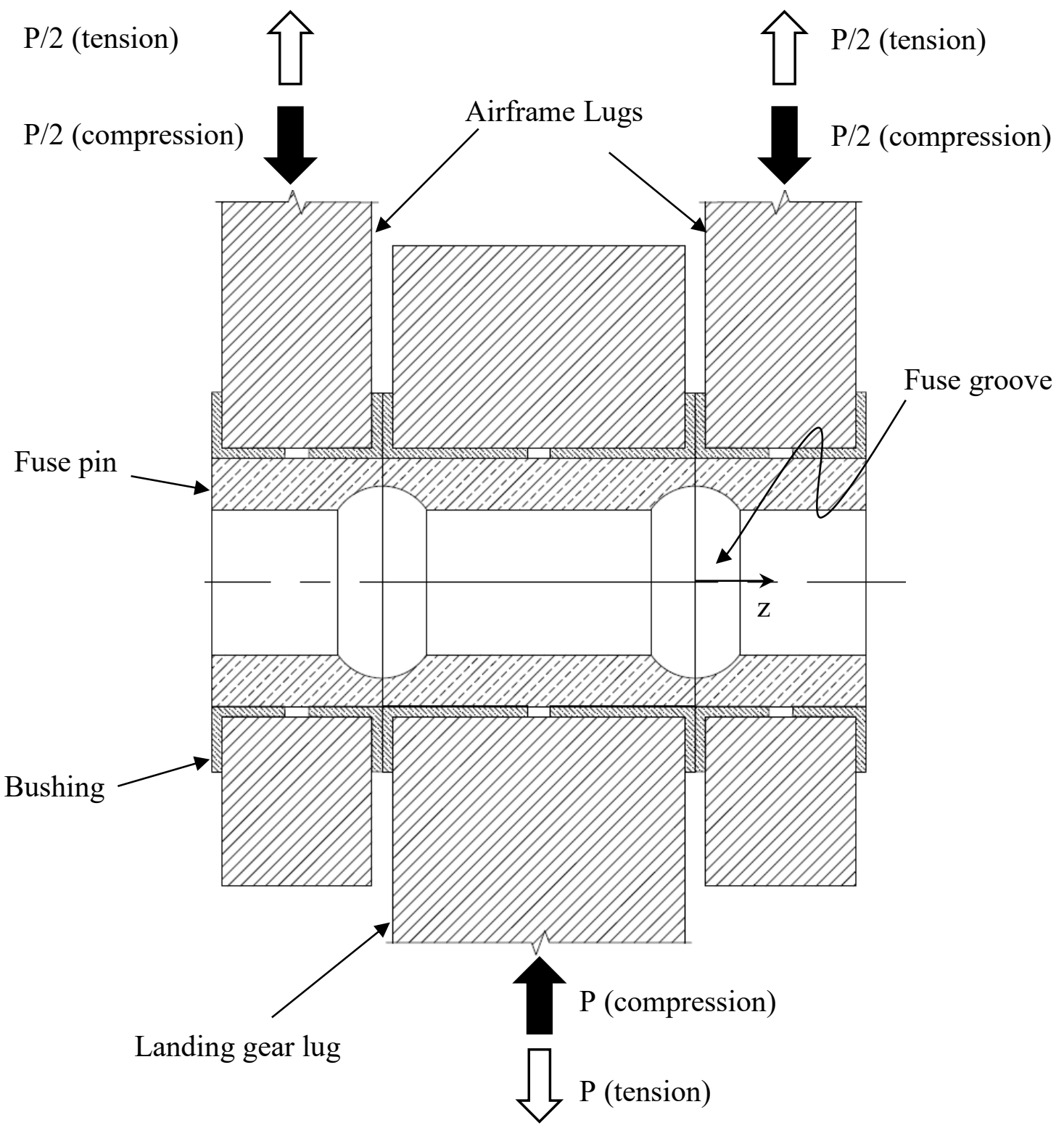

Figure 2-8: Clevis joint with bushings 


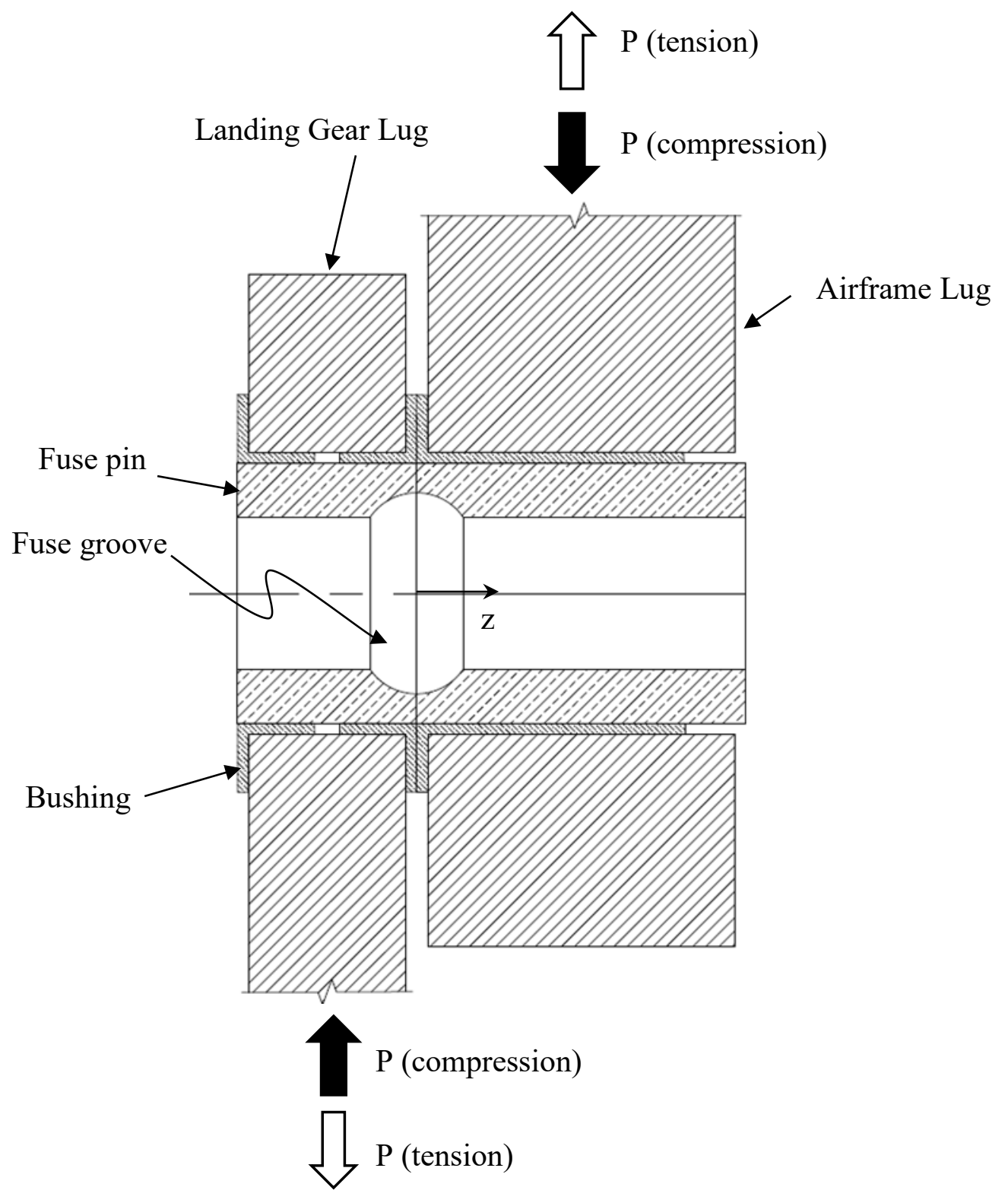

Figure 2-9: Cantilever joint with bushings 


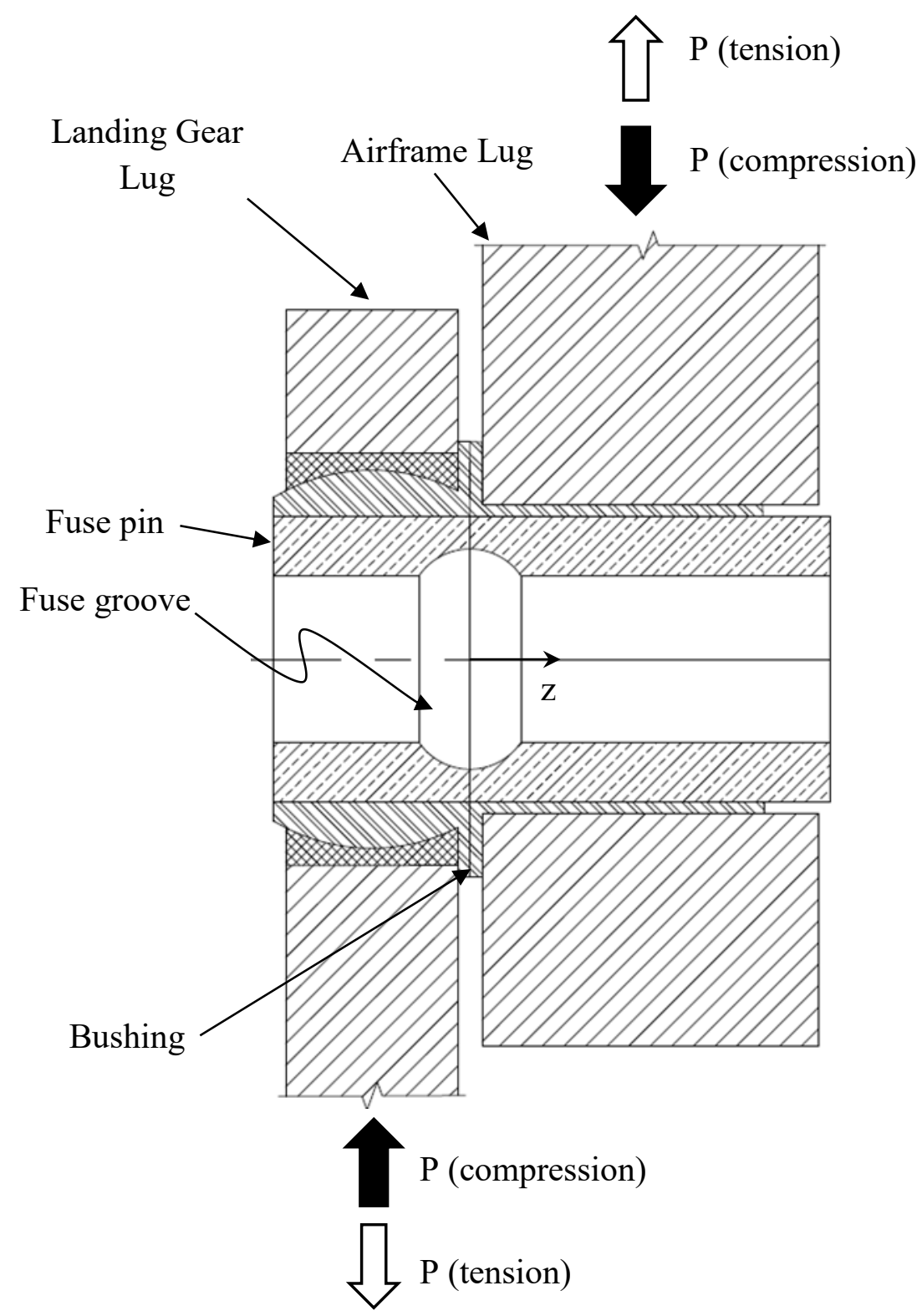

Figure 2-10: Cantilever joint with spherical bearing 


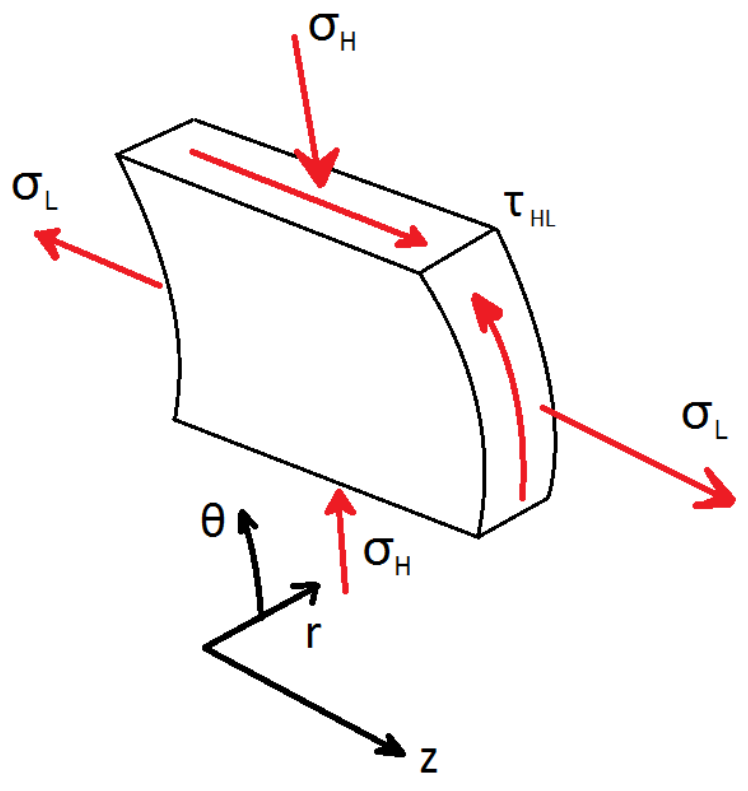

Figure 2-11: General triaxial stress state of the fuse pin groove.

\subsubsection{Fatigue Analysis of the Fuse Pin}

The fatigue analysis of the fuse pin groove has traditionally been focused on the shear failure of the pin with the maximum shear occurring at the neutral axis of the pin. To this date, uniaxial fatigue analyses using the methods introduced in Section 2.2 have been employed to predict the fatigue life of fuse pins. Methods based on pure shear analyses have been found to significantly under-predict the actual fatigue life of fuse pins when tested.

Narayan et al. (2006) proposed a simplified equivalent uniaxial fatigue stress model in an attempt to include the effects of the compressive stress as a relief on the fatigue damage in the fuse groove. This method requires the determination of a correction factor that is determined using an elastic finite element analysis of the fuse pin assembly; the correction factor is then used in a stress transformation to then reduce the biaxial hoop-shear stress state into a uniaxial equivalent stress. While it was shown that the proposed model is 
effective in predicting lower fatigue damage than the traditional uniaxial fatigue analysis methods, the method was still reported to produce significantly conservative results. More importantly, this method neglects the importance of longitudinal stresses in the fuse pin, which create a triaxial stress state instead of the simplified biaxial stress state. The longitudinal stresses due to the bending of the pin should not be neglected, even at the theoretical neutral axis of the fuse pin. In addition to this, the effects of non-proportional hardening are not accounted for when an equivalent stress approach is taken. It is these shortfalls that the work presented hereafter aims to improve on by employing the multiaxial fatigue analysis methodologies. 


\section{Chapter: Multiaxial Constitutive Model}

The constitutive model is the basis upon which the notch correction and fatigue damage models are built. With a constitutive model, elastic-plastic strains are related to applied stresses using a yield criterion, a flow rule, hardening rules. This chapter presents the implementation and verification of the Jiang-Sehitoglu multiaxial constitutive model introduced in Section 2.3.2.

\subsection{Implementation of the Jiang-Sehitoglu Incremental Plasticity Model}

A program was written in the VB .NET Framework to predict elastic-plastic strains based on input stresses. The framework was chosen so as to maintain compatibility with existing fatigue tools used by UTAS-LS. The hardening model chosen for implementation was the Jiang-Sehitoglu model, which is a modified version of the Armstrong-Frederick kinematic hardening model. The Jiang-Sehitoglu model, as discussed in Section 2.3.2, has been shown to better model behaviours such as ratchetting than more simple hardening models such as the Mroz and Garud models. While the focus of this work is not ratchetting, having the option available in the program for future research was considered to be beneficial.

\subsubsection{Approximation of Material Constants}

The Jiang-Sehitoglu model is defined by the backstress evolution given by Equation (2-40) and the plastic modulus equation (2-41). In solving these equations, three sets of material coefficients $c^{(i)}, r^{(i)}$, and $\chi^{(i)}$ are required. The material coefficients $c^{(i)}$ are used together with coefficients $r^{(i)}$ to model cyclic strain hardening. Mathematically, the coefficients $r^{(i)}$ represent the radii of each $i$ th yield surface defined by the backstress tensor $\underline{\alpha}^{(i)}$. The ratchetting decay rate is controlled by $\chi^{(i)}$ in the Jiang-Sehitoglu model. A value of $\chi^{(i)}(i=1,2, \ldots, M)=10$ was found by Shamsaei (2010) to provide sufficiently good estimates for a wide range of materials and load histories when only the steady state response is of interest. 
Jiang and Sehitoglu (1996b) presented two approaches to determining the sets of material coefficients $c^{i}$ and $r^{i}$; the method involving the pre-selection of the $c^{i}(i=1,2, \ldots, M)$ for $M$ points on the uniaxial CSS curve was chosen for the present model due to its simplicity. The following procedure is used to calculate $c^{i}$ and $r^{i}$ using a given $\Delta \sigma-\Delta \varepsilon^{p}$ curve determined from uniaxial test data:

Determining $c^{i}$ and $r^{i}$

1. Select $M$ points on the $\Delta \sigma-\Delta \varepsilon^{p}$ curve.

2. Use the following formulation to determine the material coefficients $c^{i}$ :

$$
c^{(i)}=2 \sqrt{\frac{2}{3}} \frac{1}{\Delta \varepsilon_{(i)}^{p}}(i=1,2, \ldots, M)
$$

3. Each of the material coefficients $r^{(i)}$ is the radius of the $i$ th limiting surface located by the backstress tensor $\underline{\alpha}^{(i)}$ and is calculated using Equation (3-2):

$$
r^{(i)}=\frac{2}{3} \frac{H_{(i)}-H_{(i+1)}}{c^{(i)}}(i=1,2, \ldots, M)
$$

where

$$
H_{(i)}=\frac{\Delta \sigma_{(i)}-\Delta \sigma_{(i-1)}}{\Delta \varepsilon_{(i)}^{p}-\Delta \varepsilon_{(i-1)}^{p}}(i=1,2, \ldots, M)
$$

Throughout an analysis, the material constants $c^{(i)}$ will remain constant while $r^{(i)}$ will be updated following Equation (2-46), which defines the kinematic hardening behaviour.

\section{Tanaka's nonproportionality constant}

In using Tanaka's formulation to take non-proportional hardening into account, the material constant $C_{c}$ must be selected. $C_{c}$ controls the transient behaviour associated with non-proportional hardening and sufficiently large values can cause faster convergence on maximum stress magnitudes (Zhang \& Jiang, 2008). A value of $C_{c}=200$ was used for 
all analyses on the basis that the current research is only concerned with steady-state material responses.

\subsection{Verification of the Stress-Strain Model}

Shamsaei et al. (2010) provides the in-phase (IP) and the $90^{\circ}$ out-of-phase (OP) cyclic stress-strain curve for 304L stainless steel. Using the material inputs as specified by Shamsaei et al. (2010), the in-phase and out-of-phase CSS curves were used as benchmarks for the verification of the stress-strain model. Four verification tests were performed to test the program: two single-stress monotonic tests, one proportional (in-phase) test, and one non-proportional (out-of-phase) test. The two monotonic tests are performed with the intention of producing a cyclic result; that is, they replicate a uniaxial tension-compression or uniaxial shear cyclic loading type and thus are both proportional loading. A summary of the verification tests is provided in Table 3-1.

Table 3-1: Summary of Stress-Strain Model Verification Tests

\begin{tabular}{r|l|l}
\multicolumn{1}{c|}{ Test No. } & Description & Loading Type \\
\hline 1 & Pure tension & Monotonic \\
2 & Pure shear & Monotonic \\
3 & In-phase axial-torsion & Cyclic \\
4 & $90^{\circ}$ out-of-phase axial torsion & Cyclic
\end{tabular}

To simulate a $90^{\circ}$ out-of-phase load history, a circular path of radius $\sigma_{e q}=$ $100,200,300 M P a$ traced in the $\sigma_{x}-\sqrt{3} \tau_{x y}$ stress-space was discretized as illustrated in Figure 3-1. The stress inputs for each verification step are provided in Table 3-2 and the material properties are presented in Table 3-3 for 304L stainless steel. The results of the four verification tests are plotted against the two experimental CSS curves for $304 \mathrm{~L}$ stainless steel obtained from Shamsaei et al. (2010) in Figure 3-2. 
Table 3-2: Stress inputs for Stress-Strain Model Verification Tests (stresses in MPa)

\begin{tabular}{|c|c|c|c|c|c|c|c|c|}
\hline \multirow[b]{2}{*}{ Load } & \multicolumn{2}{|c|}{$\begin{array}{ll}\text { Test } & 1 \\
\text { (Pure Tension) }\end{array}$} & \multicolumn{2}{|c|}{$\begin{array}{l}\text { Test } \\
\text { (Pure Shear) }\end{array}$} & \multicolumn{2}{|c|}{$\begin{array}{l}\text { Test } \\
\text { (In-Phase) }\end{array}$} & \multicolumn{2}{|c|}{$\begin{array}{l}\text { Test } \\
\text { (Out-of-Phase) }^{1}\end{array}$} \\
\hline & $\sigma_{x}$ & $\tau_{x y}$ & $\sigma_{x}$ & $\tau_{x y}$ & $\frac{\Delta \sigma_{x}}{2}$ & $\frac{\Delta \tau_{x y}}{2}$ & $\frac{\Delta \sigma_{x}}{2}$ & $\frac{\Delta \tau_{x y}}{2}$ \\
\hline I & 100 & 0 & 0 & 50 & 70.71 & 40.82 & 100.00 & 57.74 \\
\hline II & 200 & 0 & 0 & 100 & 141.42 & 81.65 & 200.00 & 115.47 \\
\hline III & 300 & 0 & 0 & 150 & 212.13 & 122.47 & 300.00 & 173.21 \\
\hline IV & 400 & 0 & 0 & 200 & - & - & 400.00 & 230.94 \\
\hline
\end{tabular}

Note: ${ }^{1}$ Out-of-phase loading follows a 32-load case pattern and stress amplitude values represent maximum stress amplitudes (see Figure 3-1).

Table 3-3: Material properties for 304L stainless steel (Shamsaei, Fatemi, \& Socie, 2010).

Elastic modulus, E (MPa) 195

Elastic Poissson's ratio, $v_{e}$

Yield strength, $\sigma_{y}(\mathrm{MPa})$

208

Strength coefficient, K (MPa)

680

Strain hardening exponent, $\mathrm{n}$

0.214

Cyclic Strength coefficient, $K^{\prime}(\mathrm{MPa})$

2841

Cyclic Strain hardening exponent, $n^{\prime}$

0.371

Cyclic yield strength, $\sigma_{y}^{\prime}(\mathrm{MPa})$

283 


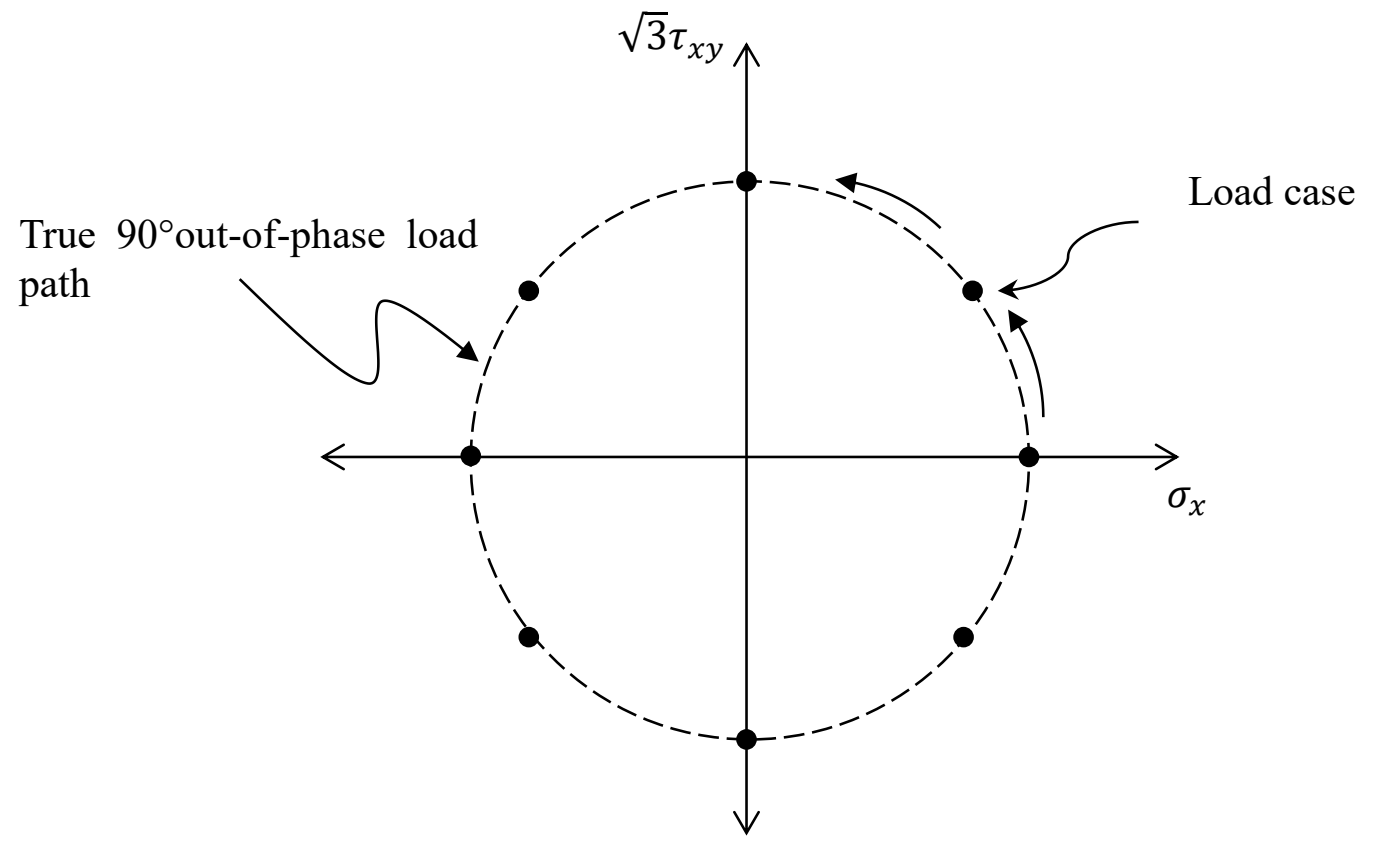

Figure 3-1: Illustration of discretization of $90^{\circ}$ out-of-phase stress path in the $\sigma_{x}-\sqrt{3} \tau_{x y}$ stress-space into individual load cases.

The purpose of the monotonic verification tests in pure tension and in pure shear is to test how well the program predicted the cyclic stress-strain behaviour from a single load case. That is, since the transient response of the material is being largely ignored the single load case should produce results akin to the steady-state response uniaxial cyclic loading of the same equivalent stress magnitude. Figure 3-2 shows the three test points for the pure tension verification test lying directly on the experimental IP CSS curve. This is expected since the IP CSS curve was generated directly from uniaxial cyclic stress-strain tests. The pure shear verification test results also follow the trend of the IP CSS curve closely. The slight deviation reflects difference in strain hardening in uniaxial shear loading and strain hardening in uniaxial tension-compression loading.

The in-phase (proportional) loading verification test results are also shown to follow the in-phase CSS curve in Figure 3-2. This is to be expected since the in-phase CSS curve is used as input into the program. More importantly, the out-of-phase (non-proportional) loading test results are also found to correlate well with the out-of-phase CSS curve. This 
verifies the implementation of both the approximation of the degree of nonproportionality $\Phi$ given by Eqn. (2-43) and the Jiang-Sehitoglu kinematic hardening model with Tanaka's nonproportionality constant.

In addition to the stress-strain results, it was found that the model was sensitive to the size of the stress increment for each step of the analysis. A user control was added to the program to allow for the specification of the maximum allowable stress increment. Much like how the element size affects the accuracy of a finite element analysis, a stress increment step that is too large can lead to erroneous results in the prediction of the plastic strain increment and the evolution of the yield surface. Through trial and error, it was found that if the allowable stress increment for calculation in the Jiang-Sehitoglu incremental plasticity model was limited to roughly $2.5 \%$ of the yield stress of the material then the analysis would converge on a solution. The convergence of the axial strain and shear strain calculated in the OP loading verification tests is presented in Figure 3-3.

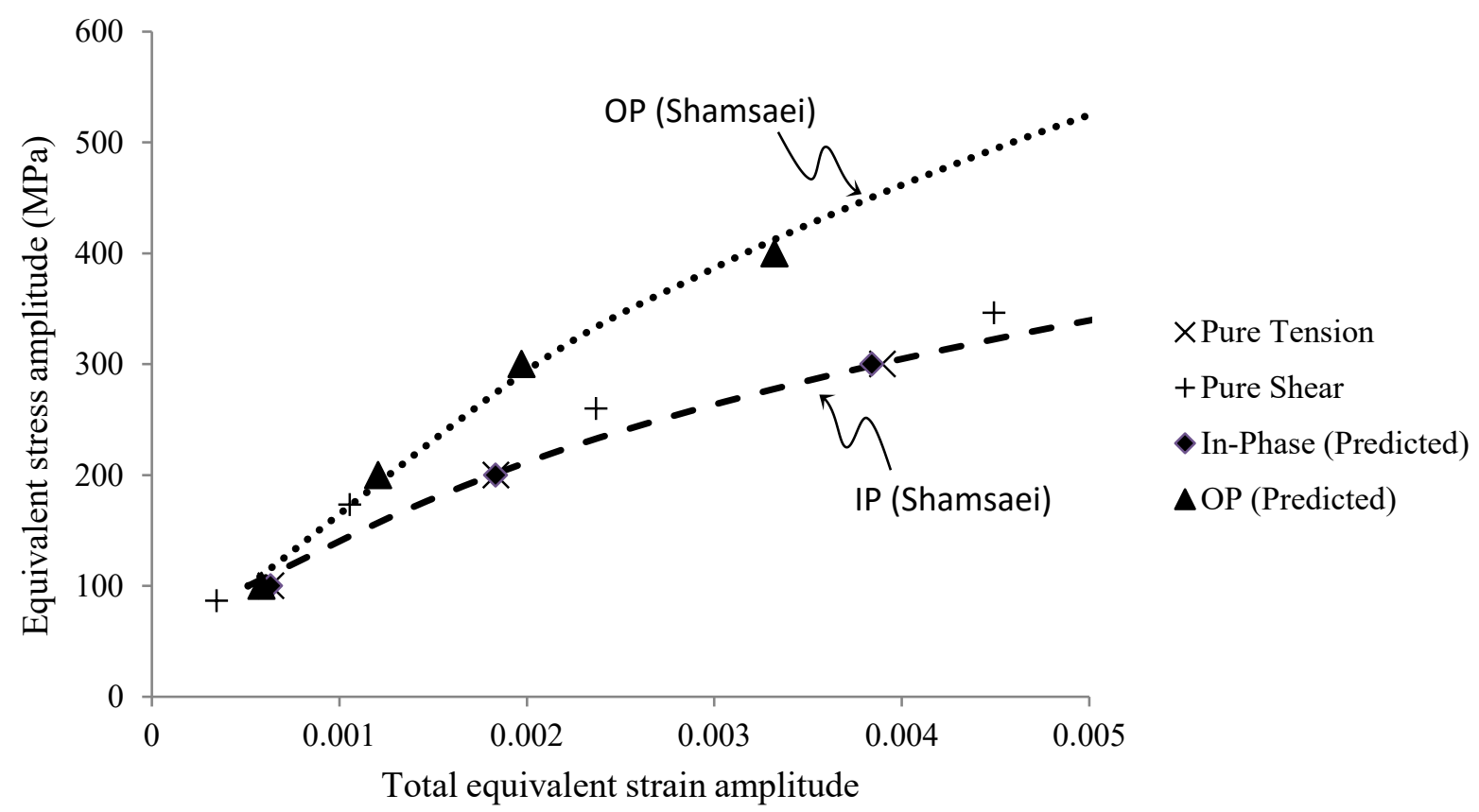

Figure 3-2: Stress-strain model verification test results compared to experimental cyclic stress-strain curves for 304L stainless steel from Shamsaei et al. (2010). 

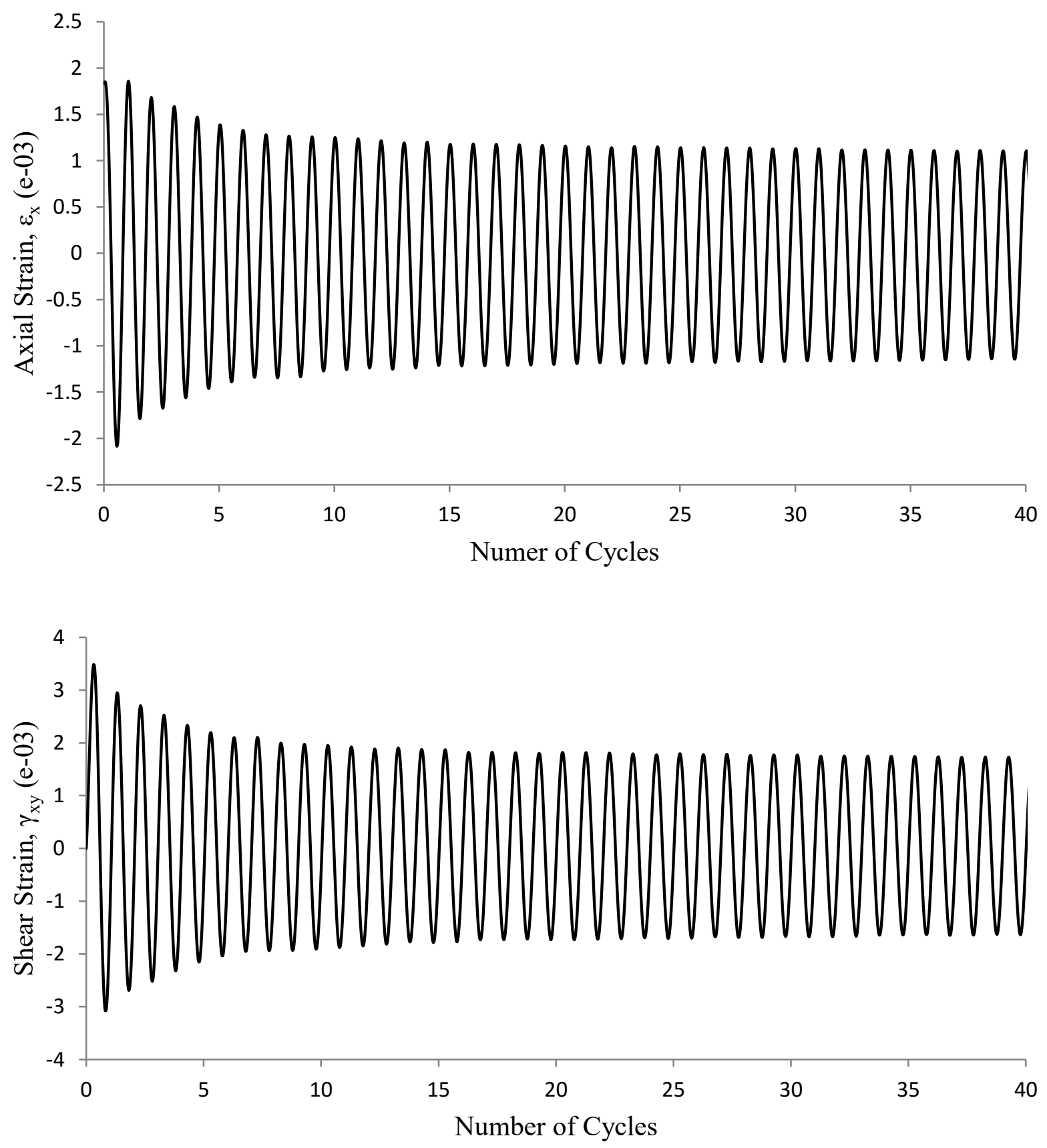

Figure 3-3: Convergence of axial and shear strain components in $90^{\circ} \mathrm{OP}$ cyclic loading verification tests. 


\section{Chapter: Multiaxial Notch Correction Methods}

\subsection{Implementation of Notch Correction Methods}

To account for the notch effect on the local stress and strain, the notch correction methods presented in Section (1.3.3) are used together with the Jiang-Sehitoglu stress-strain model. In the program, the system of equations defined by the notch correction methods is first solved to determine local notch stress and total strain increments. With the notch stress and total strain known, the local plastic strain increment as predicted by the notch correction equations can be determined. The local notch stress and plastic strain increment are then run through the JiangSehitoglu hardening model to determine if the solution is valid. The validity of the solution is determined simply on the basis of whether or not the predicted plastic strain increment is compatible with the predicted local stress increment. If the solution is not valid, the elastic stress increment is halved and the process would be restarted.

Applying the incremental ESED approach requires the use of equations (2-50), (2-52) and (2-54). Equations (2-52) and (2-54) explicitly solves for the total strain increment based on the current notch stress state determined from previous increments. The total strain increments are then used directly in (2-50) to solve for the local stress increments.

Applying the incremental Neuber's Rule and the incremental unified method requires the simultaneous solution of Eqns. (2-50),(2-59) and (2-60). These three equations are combined into one matrix equation given by Eqn. (4-1). For an analysis using the incremental Neuber's rule, a value of $C_{q}=0$ is set. For an analysis using the incremental unified method, the value of $C_{q}$ is solved for using Eqn. (2-58). 
Neuber/Unified Method System of Equations: (4-1)

$$
[A][x]=[b]
$$

$$
[A]=\left[\begin{array}{cccccccccccc}
1 & 0 & 0 & 0 & 0 & 0 & -\left(\frac{1}{E}+n_{11} K_{1}\right) & \left(\frac{v}{E}-n_{11} K_{2}\right) & \left(\frac{v}{E}-n_{11} K_{3}\right) & -n_{11} K_{4} & -n_{11} K_{5} & -n_{11} K_{6} \\
0 & 1 & 0 & 0 & 0 & 0 & \left(\frac{v}{E}-n_{22} K_{1}\right) & -\left(\frac{1}{E}+n_{22} K_{2}\right) & \left(\frac{v}{E}-n_{22} K_{3}\right) & -n_{22} K_{4} & -n_{22} K_{5} & -n_{22} K_{6} \\
0 & 0 & 1 & 0 & 0 & 0 & \left(\frac{v}{E}-n_{33} K_{1}\right) & \left(\frac{v}{E}-n_{33} K_{2}\right) & -\left(\frac{1}{E}+n_{33} K_{3}\right) & -n_{33} K_{4} & -n_{33} K_{5} & -n_{33} K_{6} \\
0 & 0 & 0 & 1 & 0 & 0 & -n_{12} K_{1} & -n_{12} K_{2} & -n_{12} K_{3} & -\left(\frac{1+v}{E}+n_{12} K_{4}\right) & -n_{12} K_{5} & -n_{12} K_{6} \\
0 & 0 & 0 & 0 & 1 & 0 & -n_{23} K_{1} & -n_{23} K_{2} & -n_{23} K_{3} & -n_{23} K_{4} & -\left(\frac{1+v}{E}+n_{23} K_{5}\right) & -n_{23} K_{6} \\
0 & 0 & 0 & 0 & 0 & 1 & -n_{31} K_{1} & -n_{31} K_{2} & -n_{31} K_{3} & -n_{31} K_{4} & -n_{31} K_{5} & -\left(\frac{1+v}{E}+n_{31} K_{6}\right) \\
Q_{1} \sigma_{11}^{U} & 0 & 0 & 0 & 0 & 0 & Q_{2} \varepsilon_{11}^{U} & 0 & 0 & 0 & 0 & 0 \\
0 & Q_{1} \sigma_{22}^{U} & 0 & 0 & 0 & 0 & 0 & Q_{2} \varepsilon_{22}^{U} & 0 & 0 & 0 & 0 \\
0 & 0 & Q_{1} \sigma_{33}^{U} & 0 & 0 & 0 & 0 & 0 & Q_{2} \varepsilon_{33}^{U} & 0 & 0 & 0 \\
0 & 0 & 0 & Q_{1} \sigma_{12}^{U} & 0 & 0 & 0 & 0 & 0 & 0 & 0 & 0 \\
0 & 0 & 0 & 0 & Q_{1} \sigma_{23}^{U} & 0 & 0 & 0 & 0 & Q_{2} \varepsilon_{12}^{U} & 0 & 0 \\
Q_{1} \sigma_{11}^{U} & Q_{1} \sigma_{22}^{U} & Q_{1} \sigma_{33}^{U} & 2 Q_{1} \sigma_{12}^{U} & 2 Q_{1} \sigma_{23}^{U} & 2 Q_{1} \sigma_{31}^{U} & Q_{2} \varepsilon_{11}^{U} & Q_{2} \varepsilon_{22}^{U} & Q_{2} \varepsilon_{33}^{U} & 2 Q_{2} \varepsilon_{12}^{U} & 2 Q_{2} \varepsilon_{23}^{U} & 2 Q_{2} \varepsilon_{31}^{U}
\end{array}\right]
$$

$$
[b]=\left[\begin{array}{llllllllllll}
0 & 0 & 0 & 0 & 0 & 0 & \psi_{11}^{e} & \psi_{22}^{e} & \psi_{33}^{e} & \psi_{12}^{e} & \psi_{23}^{e} & \Gamma^{e}
\end{array}\right]^{T}
$$

$$
[x]=\left[\begin{array}{llllllllllll}
d \varepsilon_{11}^{U} & d \varepsilon_{22}^{U} & d \varepsilon_{33}^{U} & d \varepsilon_{12}^{U} & d \varepsilon_{23}^{U} & d \varepsilon_{13}^{U} & d \sigma_{11}^{U} & d \sigma_{22}^{U} & d \sigma_{33}^{U} & d \sigma_{12}^{U} & d \sigma_{23}^{U} & d \sigma_{13}^{U}
\end{array}\right]^{T}
$$$$
\psi_{\alpha \beta}^{e}=\sigma_{\alpha \beta}^{e} d \varepsilon_{\alpha \beta}^{e}+\epsilon_{\alpha \beta}^{e} d \sigma_{\alpha \beta}^{e}, \quad \Gamma^{e}=\sigma_{i j}^{e} d \varepsilon_{i j}^{e}+\epsilon_{i j}^{e} d \sigma_{i j}^{e}
$$

$$
Q_{1}=1+C_{q}, \quad Q_{2}=1-C_{q}
$$

$K_{i}(i=1,2,3, \ldots, 6)$ determined as presented Equation (2-50). 


\subsection{Verification of Proportional Loading of Notch Specimens}

The verification tests presented in this section allow for the testing of the notch analysis methods without considering any non-proportional hardening effects. Finite element analyses are used as a benchmark to compare the performance of the three multiaxial notch correction methods (ESED, Neuber, and Unified) presented in the previous section. It is the objective of this section to establish an understanding of how well the notch correction methods as implemented will perform for pure axial, pure shear, and axial-torsion load histories and the effect of geometry and material on the quality of predictions.

\subsubsection{Finite Element Analysis of Notched Specimens}

Finite element analyses (FEA) of three different specimens were performed using ABAQUS to form a benchmark for the numerical analysis performed by the stress-strain program with the multiaxial notch correction methods. The geometry of the specimens used is shown in Figure 4-1. The notch geometry and material is varied for the three different analyses as per Table 4-1. Two different geometries were selected to represent different stress concentration intensities. SAE 1070 and 1045 steel were selected for analysis based on available data from the literature; the material properties are summarized in Table 4-2.

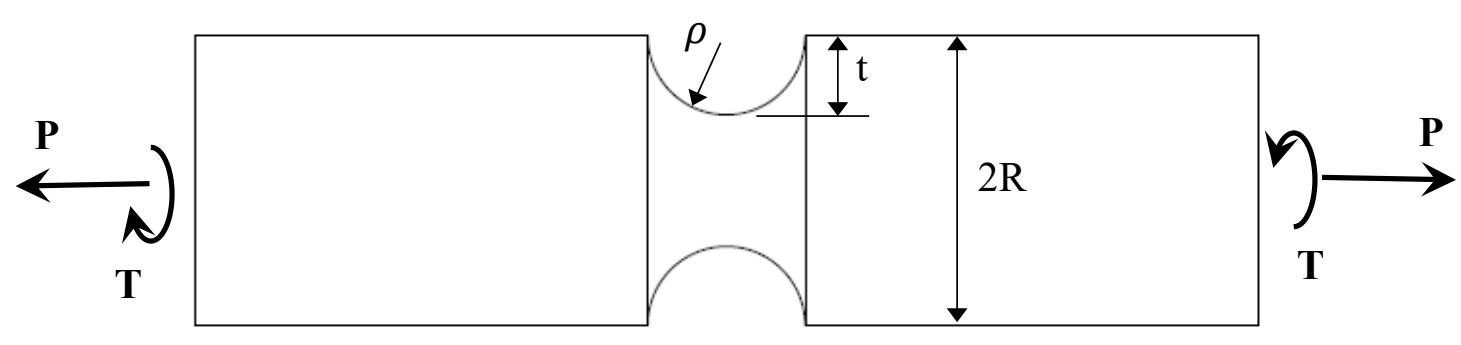

Figure 4-1: Geometry of notched specimen for numerical analysis. 
Table 4-1: Geometry of specimens used for verification of multiaxial notch analysis.

\begin{tabular}{|r|l|r|r|r|l|}
\hline Specimen & Material & \multicolumn{1}{c|}{$\rho / t$} & $R / t$ & $K_{t, a x}$ & $K_{t, t}$ \\
\hline \hline A & SAE 1070 & 0.5 & 1 & 1.54 & 1.13 \\
\hline B & SAE 1070 & 0.3 & 7 & 3.66 & 2.19 \\
\hline C & SAE 1045 & 0.3 & 7 & 3.66 & 2.19 \\
\hline
\end{tabular}

Table 4-2: Material properties for SAE 1070 (Firat, 2011) and SAE 1045 (Singh, Glinka, \& Dubey, 1996).

\begin{tabular}{|l|r|r|}
\hline Material & SAE 1070 & SAE 1045 \\
\hline \hline Elastic modulus, E $(\mathrm{GPa})$ & 210 & 202 \\
\hline Poisson's ratio, $v$ & 0.3 & 0.3 \\
\hline Yield strength, $\sigma_{y}(\mathrm{MPa})$ & 242 & 202 \\
\hline Cyclic strength coefficient, $K^{\prime}$ & 1736 & 1258 \\
\hline Cyclic strain hardening exponent, $n^{\prime}$ & 0.199 & 0.208 \\
\hline
\end{tabular}

For each specimen, three different load types at three load levels were considered: pure axial loading, pure torsional loading, and proportional axial-torsional loading. Nonproportional loading was not considered for the FEA since non-proportional hardening is not accounted for in the standard static analysis in ABAQUS. The loads associated with each test case are summarized in Table 4-3. The specimen A loads were chosen first such that there were three levels of load corresponding to nominal stresses of $\sigma_{e q}=400 \mathrm{MPa}$, , 446.5 MPa, and 600 MPa. The second load levels of each test (tests 1B, 2B, and 3B) were selected initially as a baseline reference from Firat (2011), while the first and third levels were chosen arbitrarily to represent a relatively low and high load. 
With the loads chosen for specimen A, loads were chosen for Specimen B/C so that the resulting elastic axial notch stresses were the same as those in specimen A; this way for each test the effect of the notch, and not the stress level, can be compared. To determine the specimen $\mathrm{B} / \mathrm{C}$ loads, a trial-and-error approach was required due to yielding at the notch. To provide an initial guess, the theoretical stress concentration factors from Table 4-1 were used. It is noted that while the axial notch stresses are matched, the circumferential notch stresses will not be the same between the two specimens due to the difference in the ratio of the stress concentration factors in the axial and circumferential directions.

Table 4-3: End loads applied to finite element analyses for notch verification tests.

\begin{tabular}{|r|r|r|r|r|}
\hline & & Specimen A & Specimen B/C \\
\hline \hline Test & $\mathrm{P}(\mathrm{kN})$ & $\mathrm{T}(\mathrm{N}-\mathrm{m})$ & $\mathrm{P}(\mathrm{kN})$ & $\mathrm{T}(\mathrm{N}-\mathrm{m})$ \\
\hline \hline $1 \mathrm{a}$ & 202.7 & 0 & 213.6 & 0 \\
\hline $1 \mathrm{~b}$ & 226.2 & 0 & 238.5 & 0 \\
\hline $1 \mathrm{c}$ & 304.0 & 0 & 320.4 & 0 \\
\hline $2 \mathrm{a}$ & 0 & 743.1 & 0 & 1925 \\
\hline $2 \mathrm{~b}$ & 0 & 829.5 & 0 & 1441 \\
\hline $2 \mathrm{c}$ & 0 & 1114.6 & 0 & 2887 \\
\hline $3 \mathrm{a}$ & 143.3 & 525.5 & 141.6 & 1441 \\
\hline $3 \mathrm{~b}$ & 150.0 & 621.0 & 158.1 & 1608 \\
\hline $3 \mathrm{c}$ & 215.0 & 788.1 & 212.4 & 2161 \\
\hline
\end{tabular}

Quadratic quadrilateral elements used throughout each axisymmetric FEA. The meshing scheme is as shown in Figure 4-2. The mesh was refined at the notch until a minimum of $10 \%$ convergence was achieved. To further illustrate the contrast in the sharpness of the notch, the meshed specimen notches are compared in Figure 4-3. 


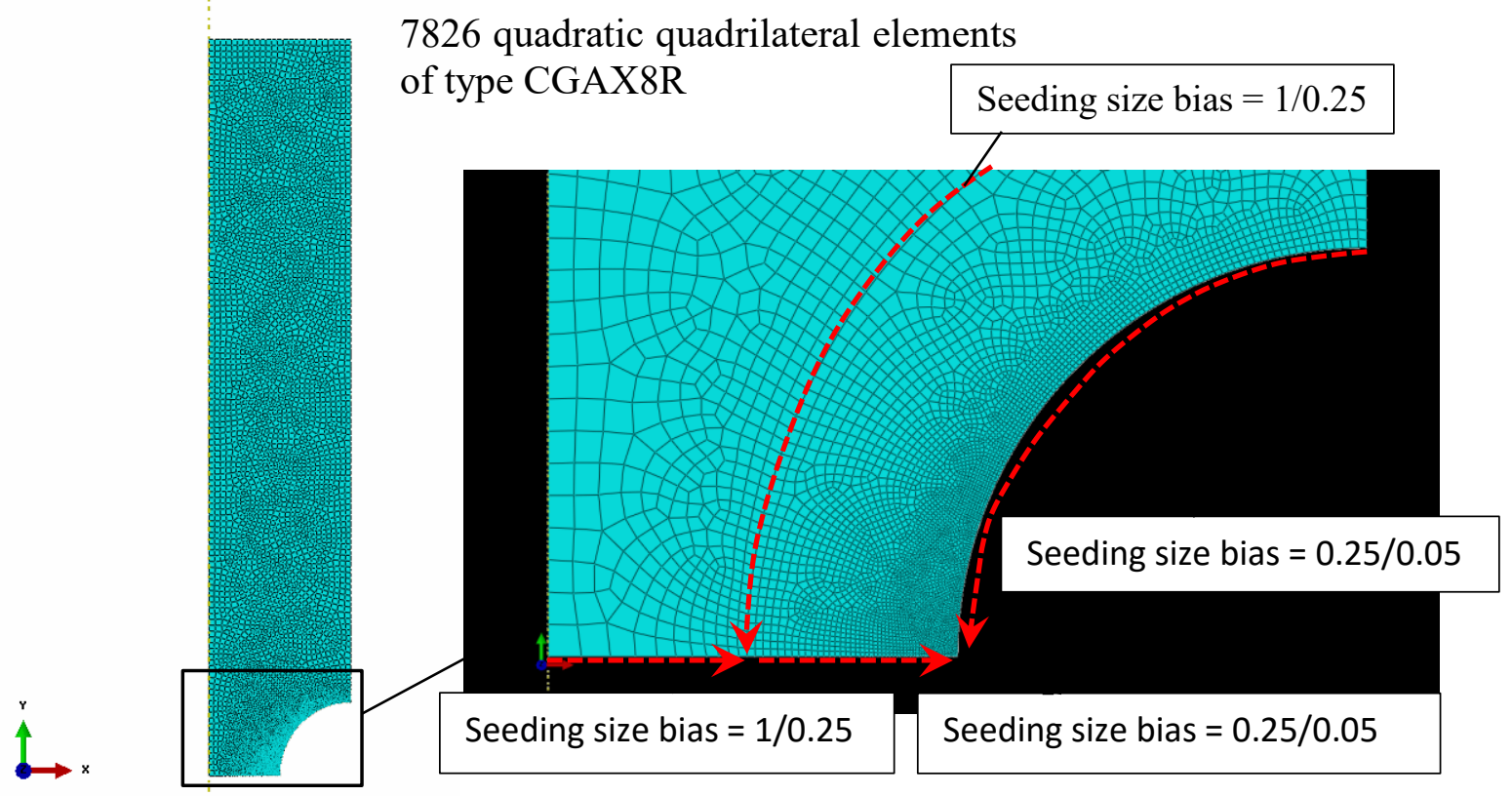

Figure 4-2: Axisymmetric finite element analysis of axial-torsion specimens. Mesh refined based on convergence study.

Specimen A

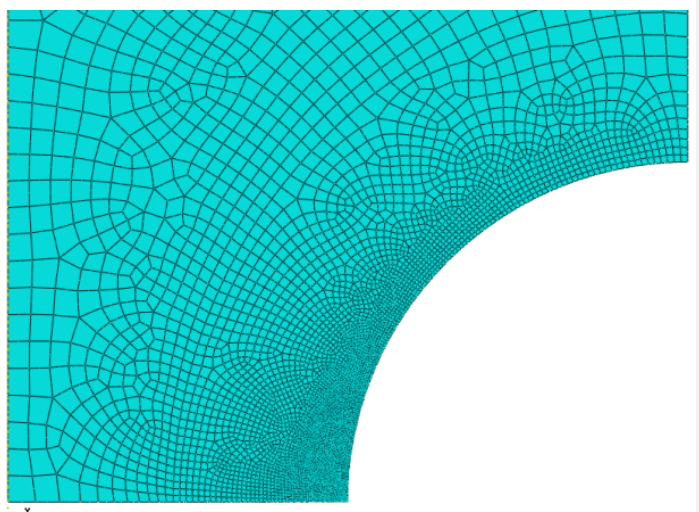

$\stackrel{4}{\circ}$
Specimen B \& C

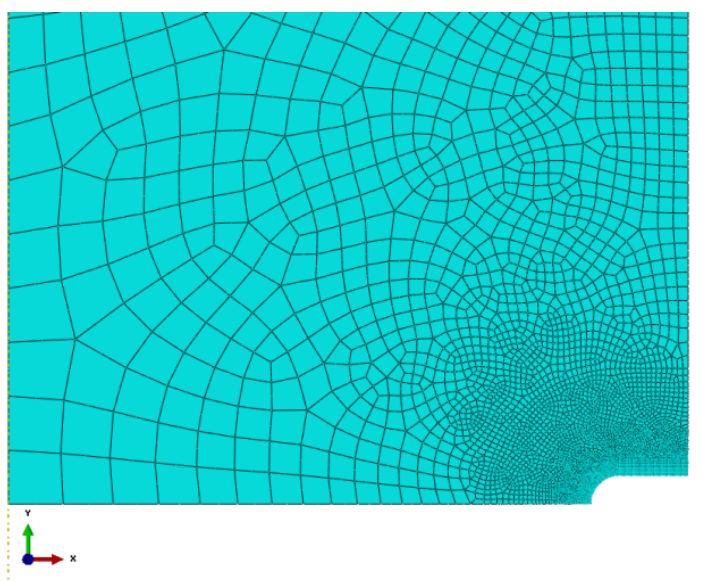

Figure 4-3: Meshed notches. 


\subsubsection{Results and Discussion}

To verify the implementation of the notch analysis with the multiaxial stress-strain program, the results of the FEA defined in Section 4.2.1.1 were compared to results produced using the incremental ESED, Neuber, and Unified notch correction methods. Elastic stresses as predicted using theoretical stress concentration factors or elastic FEA are used as input into these notch correction methods. The input stresses for the notch correction methods for each test and specimen are presented in Table 4-4. The resulting notch stresses predicted by the notch correction methods are plotted against the FEA predicted stresses in Figure 4-4 to Figure 4-6, Figure 4-7 to Figure 4-9, and Figure 4-10 to Figure 4-12 to for the pure axial tests, pure torsion tests, and axial-torsion tests, respectively.

Considering the results of the pure axial tests for Specimen A in Figure 4-4, a clear decrease in correlation with FEA results is observed for the axial and hoop stresses with increasing load level. With the exception of the hoop stresses predicted by the multiaxial Neuber's rule, most predictions are within $10-15 \%$ of the FEA values. Comparing these results to those for Specimen B in Figure 4-5 one can see significant improvements in correlation with FEA due to the increase in stress concentration. The increased $K_{t}$ creates and sustains a more concentrated and localized plastic zone as the load increases. This is important for the application of the incremental ESED, Neuber's rule, and Unified notch correction method because of the assumption of conserved virtual strain energy. A lower $\mathrm{K}_{\mathrm{t}}$ allows for yielding to occur over a larger area, and thus reducing the validity of the conserved virtual strain energy assumption. Alternatively, increasing the ductility of a material will also result in a larger plastic zone for the same geometry. The effect of increased ductility is observed by comparing the results of Specimen C in Figure 4-5 to those of Specimen B. While the hoop stress predictions are better than those predicted for Specimen A, severe reductions in correlation are observed for the axial and hoop stresses at high load level.

The pure torsion verification tests for the three notched specimens show similar results to that of the pure axial tests. With higher load, an overall reduction in correlation is observed 
with the exception of the highly notched Specimen B. Overall the shear stresses predicted by the notch correction methods are less than those predicted by the FEA by $5-15 \%$. Specimen $\mathrm{C}$, with the highest ductility and highest $\mathrm{K}_{\mathrm{t}}$, resulted in the best predictions for low- and mid-level loads but the worst predictions for the high-level load.

For the axial-torsion verification tests, it is expected that the trends observed in the pure axial and pure torsion verification tests would persist. Figure 4-10 to Figure 4-12 compares all three stress components on each plot for each of the notched specimens. Specimen B is observed to provide the best correlation with the FEA results for all three components of stress and demonstrated the ability to sustain good correlation for higher load levels. Most predictions for Specimen B are within 10\% difference of the FEA predictions and only a few low-level load predictions ended up with 10-20\% difference. Hoop stress predictions for Specimen A continued to diverge significantly from experimental results when predicted by the multiaxial Neuber's rule, and axial and shear predictions would correlate less with the FEA results with increasing load for all notch correction methods. The hoop stresses predicted for Specimen $\mathrm{C}$ did not appear to be affected much by the increased ductility in the axial-torsion tests; however, the axial stresses continued to be overpredicted by the notch correction methods.

Overall, predictions are within $20 \%$ difference as compared to the FEA results indicating reasonable performance by the implementation of the incremental ESED, Neuber, and Unified approaches. The increase in correlation with FEA results corresponds to the increased localization of the stress at the stress concentration. Since these notch correction methods rely on the assumption of a localized plastic zone to use the conservation of virtual strain energy, the predictions will deviate further from the real results as the plastic zone grows larger. The localization of the plastic zone breaks down for lower Kt where a larger section is subjected to yielding and for more ductile materials such as SAE 1045. Figure 4-13 compares the ratio of the plastic zone size to the overall radius of the notched section of the three specimens at the three load levels. Specimen A, with the lower $\mathrm{K}_{\mathrm{t}}$, has the largest plastic zone size by orders of magnitude compared to the other two specimens. With the same material, Specimen B has a much smaller plastic zone size for all three load 
levels due to the roughly doubled stress concentration factor. Specimen $\mathrm{C}$ has a plastic zone size of about an order of magnitude more than Specimen B for all load levels due to the increased ductility. Based on the observations discussed previously, it can be concluded that these incremental multiaxial notch correction methods perform better with increasing stress concentration and decreasing ductility.

Table 4-4: Elastic notch stresses (MPa) used as input into stress-strain model for notch verification tests.

\begin{tabular}{|r|r|r|r|r|r|r|}
\hline \multirow{2}{*}{ Test } & \multicolumn{3}{|c|}{ Specimen A } & \multicolumn{3}{c|}{ Specimen B/C } \\
\cline { 2 - 7 } & \multicolumn{1}{|c|}{$\sigma_{x}$} & $\sigma_{y}$ & \multicolumn{1}{c|}{$\tau_{x y}$} & \multicolumn{1}{c|}{$\sigma_{x}$} & \multicolumn{1}{c|}{$\sigma_{y}$} & \multicolumn{1}{c|}{$\tau_{x y}$} \\
\hline $1 \mathrm{a}$ & 564.9 & 104.4 & 0 & 564.9 & 151.2 & 0 \\
\hline $1 \mathrm{~b}$ & 630.5 & 116.5 & 0 & 630.5 & 168.8 & 0 \\
\hline $1 \mathrm{c}$ & 759.1 & 140.2 & 0 & 759.1 & 203.2 & 0 \\
\hline $2 \mathrm{a}$ & 0 & 0 & -265.2 & 0 & 0 & -265.2 \\
\hline $2 \mathrm{~b}$ & 0 & 0 & -295.9 & 0 & 0 & -295.9 \\
\hline $2 \mathrm{c}$ & 0 & 0 & -356.3 & 0 & 0 & -356.3 \\
\hline $3 \mathrm{a}$ & 374.5 & 69.2 & -198.5 & 374.5 & 100.3 & -198.5 \\
\hline $3 \mathrm{~b}$ & 418.0 & 77.2 & -221.6 & 418.0 & 111.9 & -221.6 \\
\hline $3 \mathrm{c}$ & 503.2 & 93.0 & -266.7 & 503.2 & 134.7 & -266.7 \\
\hline
\end{tabular}




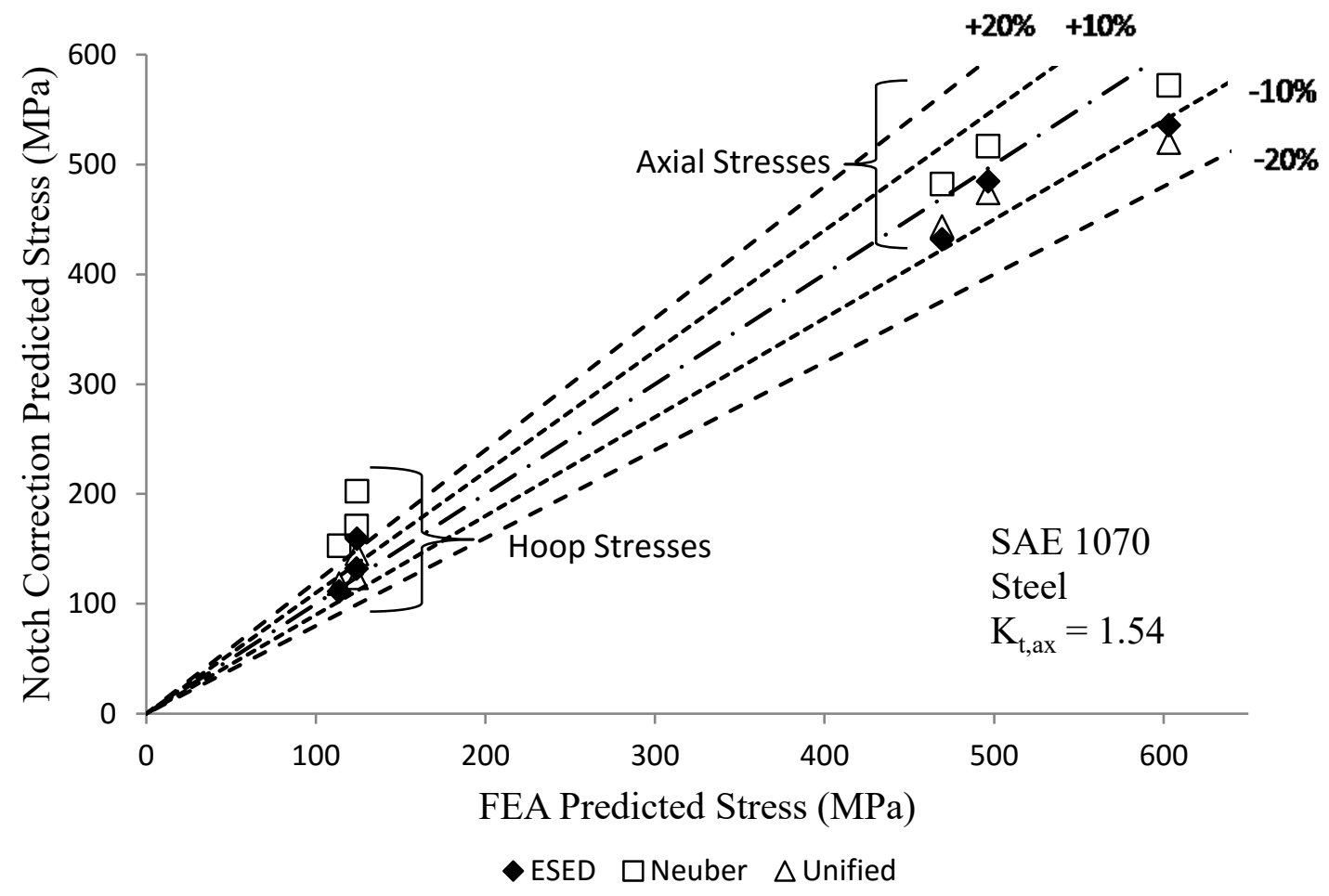

Figure 4-4: Comparison of notch correction and FEA stress predictions for Specimen A pure axial tests $(1 \mathrm{a}, 1 \mathrm{~b}, 1 \mathrm{c})$. 


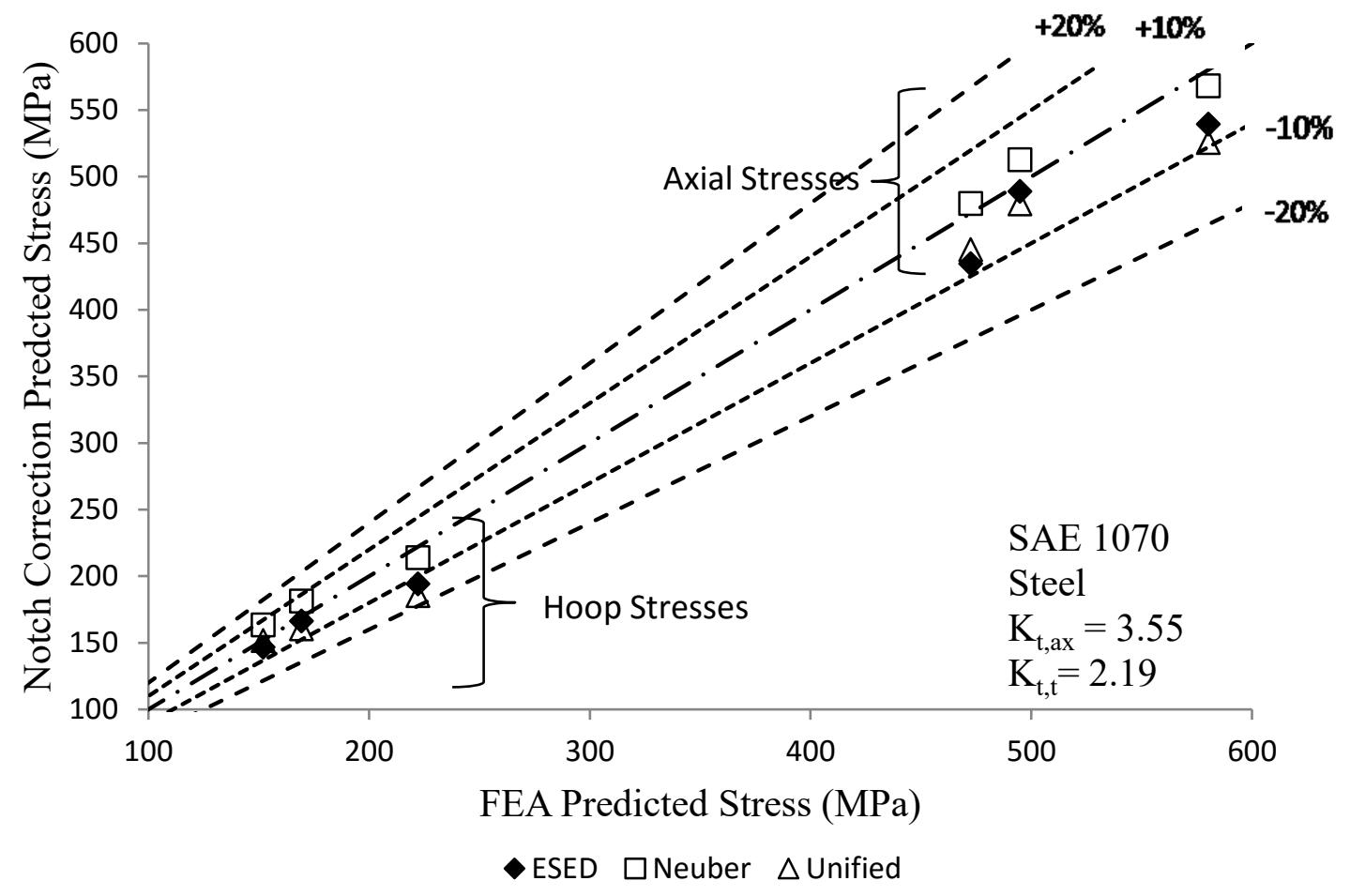

Figure 4-5: Comparison of notch correction and FEA stress predictions for Specimen B pure axial tests $(1 \mathrm{a}, 1 \mathrm{~b}, 1 \mathrm{c})$. 


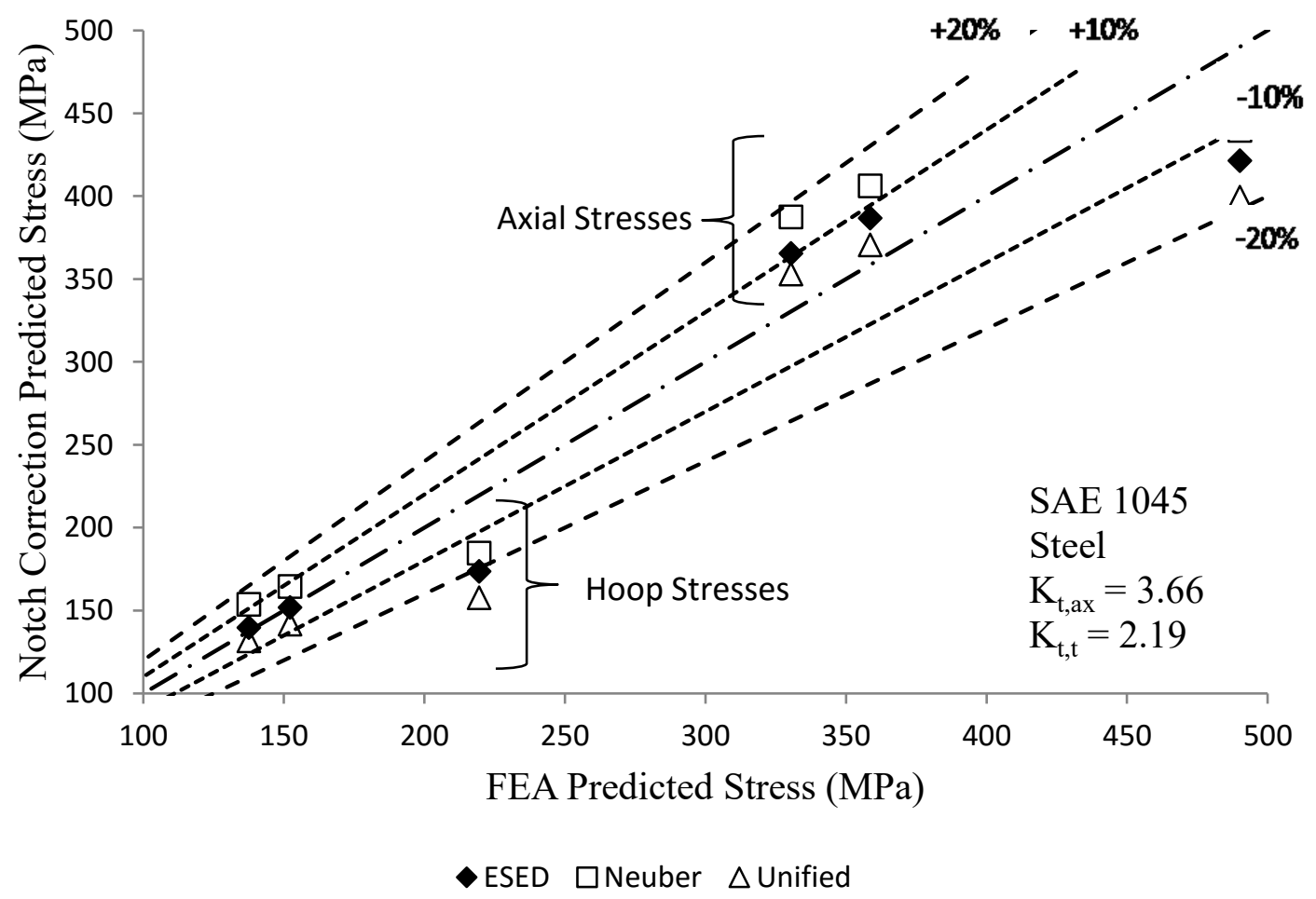

Figure 4-6: Comparison of notch correction and FEA stress predictions for Specimen C pure axial tests $(1 \mathrm{a}, 1 \mathrm{~b}, 1 \mathrm{c})$. 


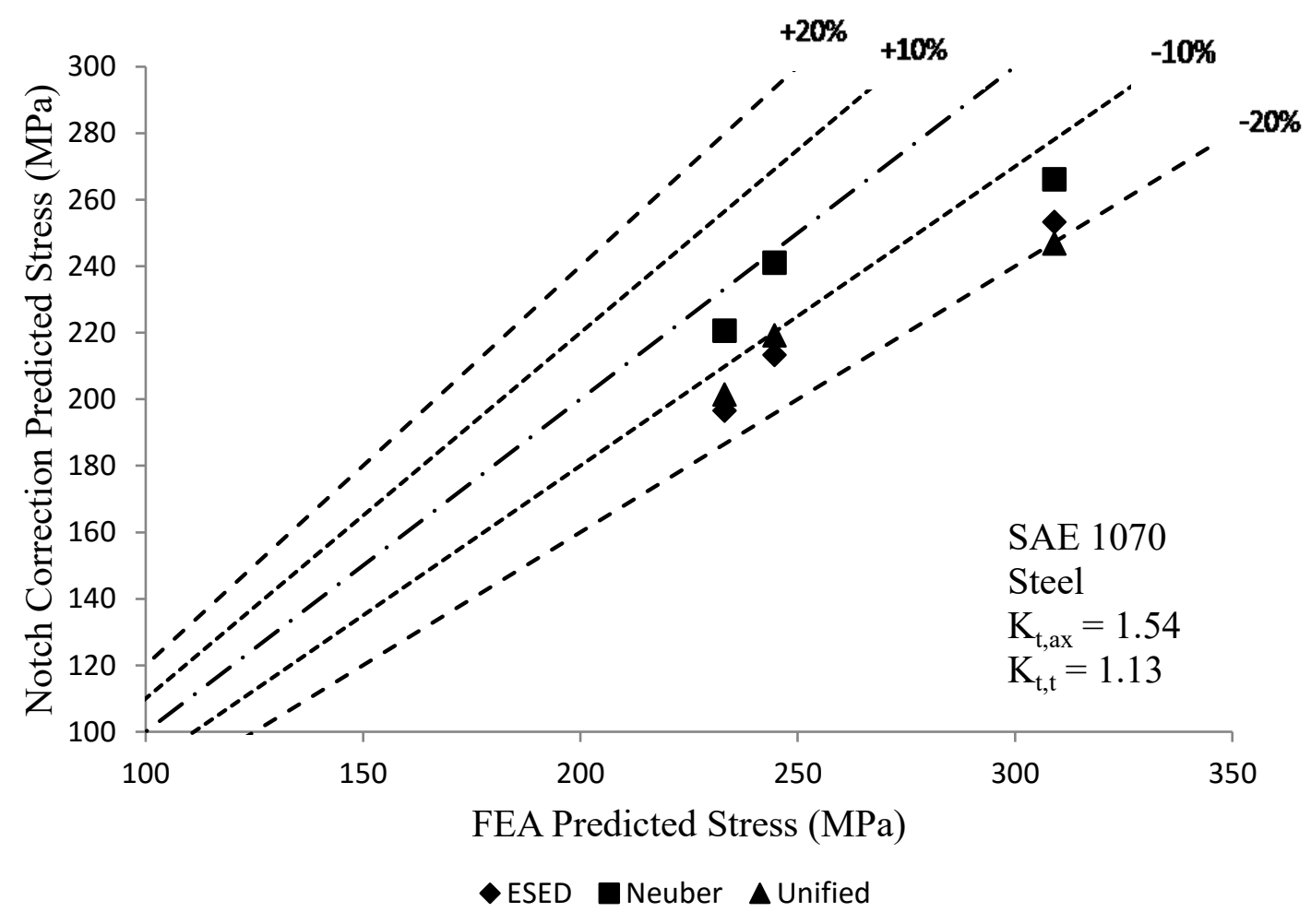

Figure 4-7: Comparison of notch correction and FEA stress predictions for Specimen A pure torsion tests $(2 \mathrm{a}, 2 \mathrm{~b}, 2 \mathrm{c})$. 


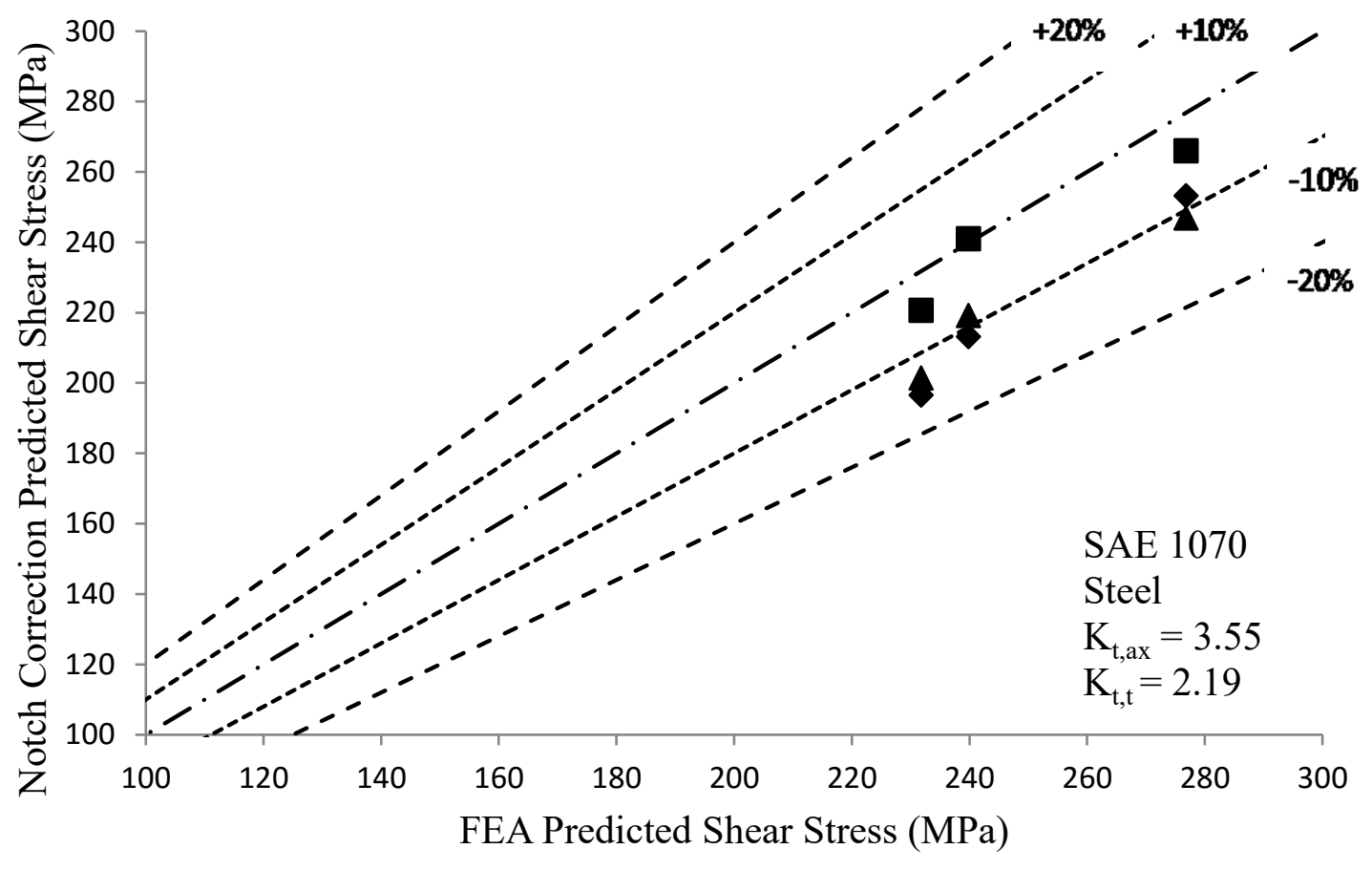

$\bullet$ ESED $\boldsymbol{\square}$ Neuber $\Delta$ Unified

Figure 4-8: Comparison of notch correction and FEA stress predictions for Specimen B pure torsion tests $(2 \mathrm{a}, 2 \mathrm{~b}, 2 \mathrm{c})$. 


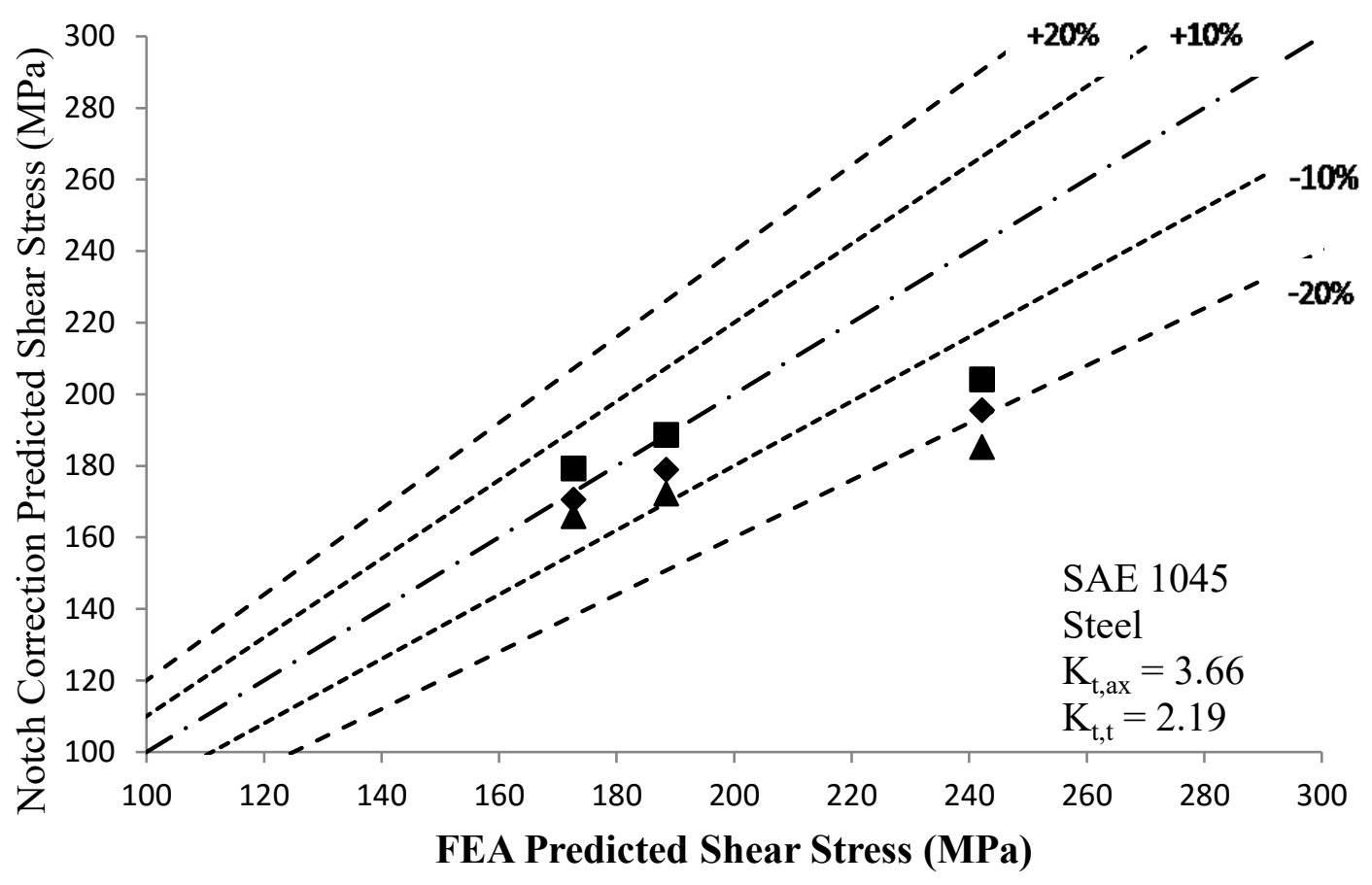

$\bullet$ ESED (Shear) $\quad$ Neuber (Shear) $\Delta$ Unified (Shear)

Figure 4-9: Comparison of notch correction and FEA stress predictions for Specimen C pure torsion tests $(2 \mathrm{a}, 2 \mathrm{~b}, 2 \mathrm{c})$. 


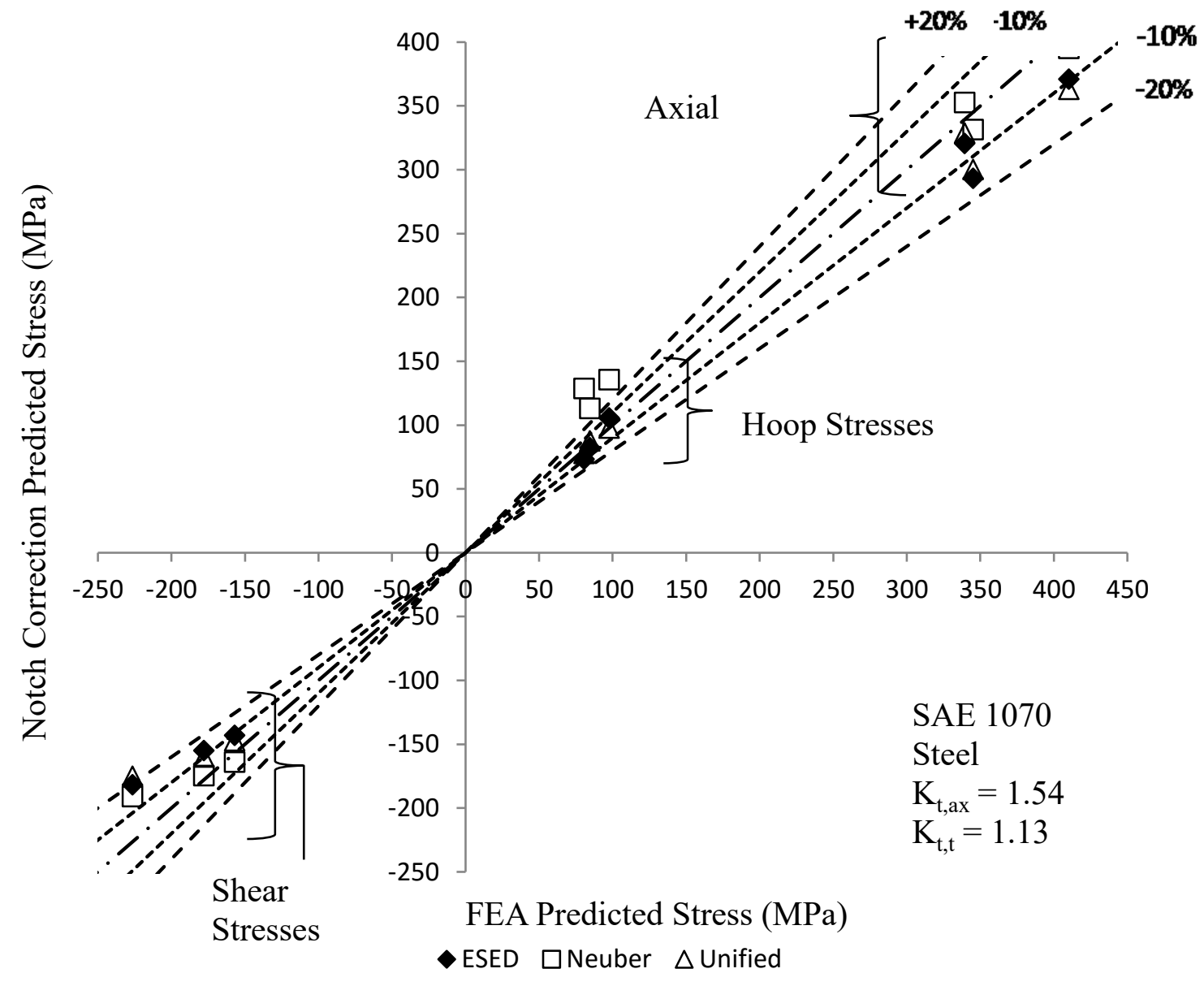

Figure 4-10: Comparison of notch correction and FEA stress predictions for Specimen A axial-torsion tests (3a, 3b, 3c). 


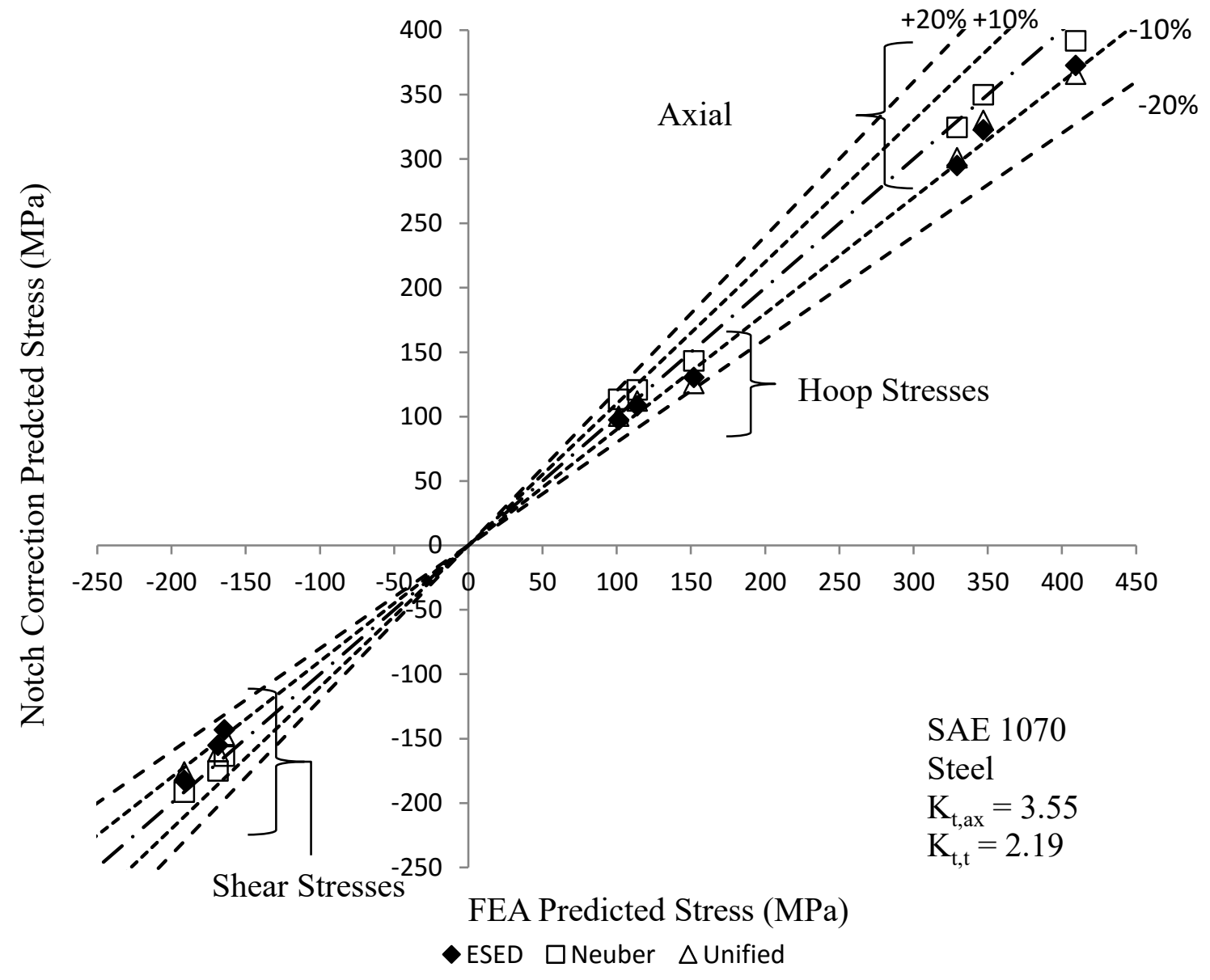

Figure 4-11: Comparison of notch correction and FEA stress predictions for Specimen B axial-torsion tests (3a, 3b, 3c). 


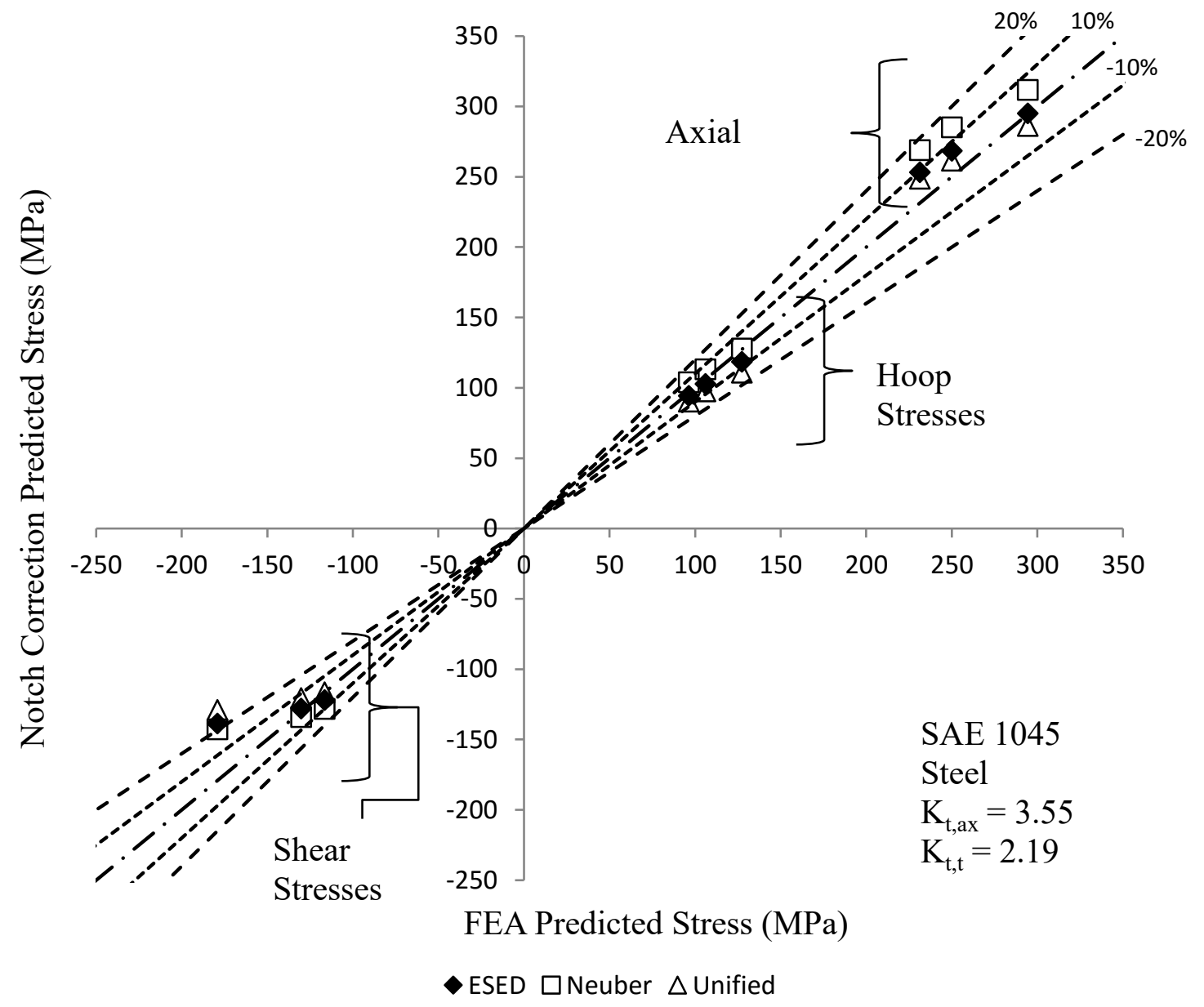

Figure 4-12: Comparison of notch correction and FEA stress predictions for Specimen C axial-torsion tests (3a, 3b, 3c). 


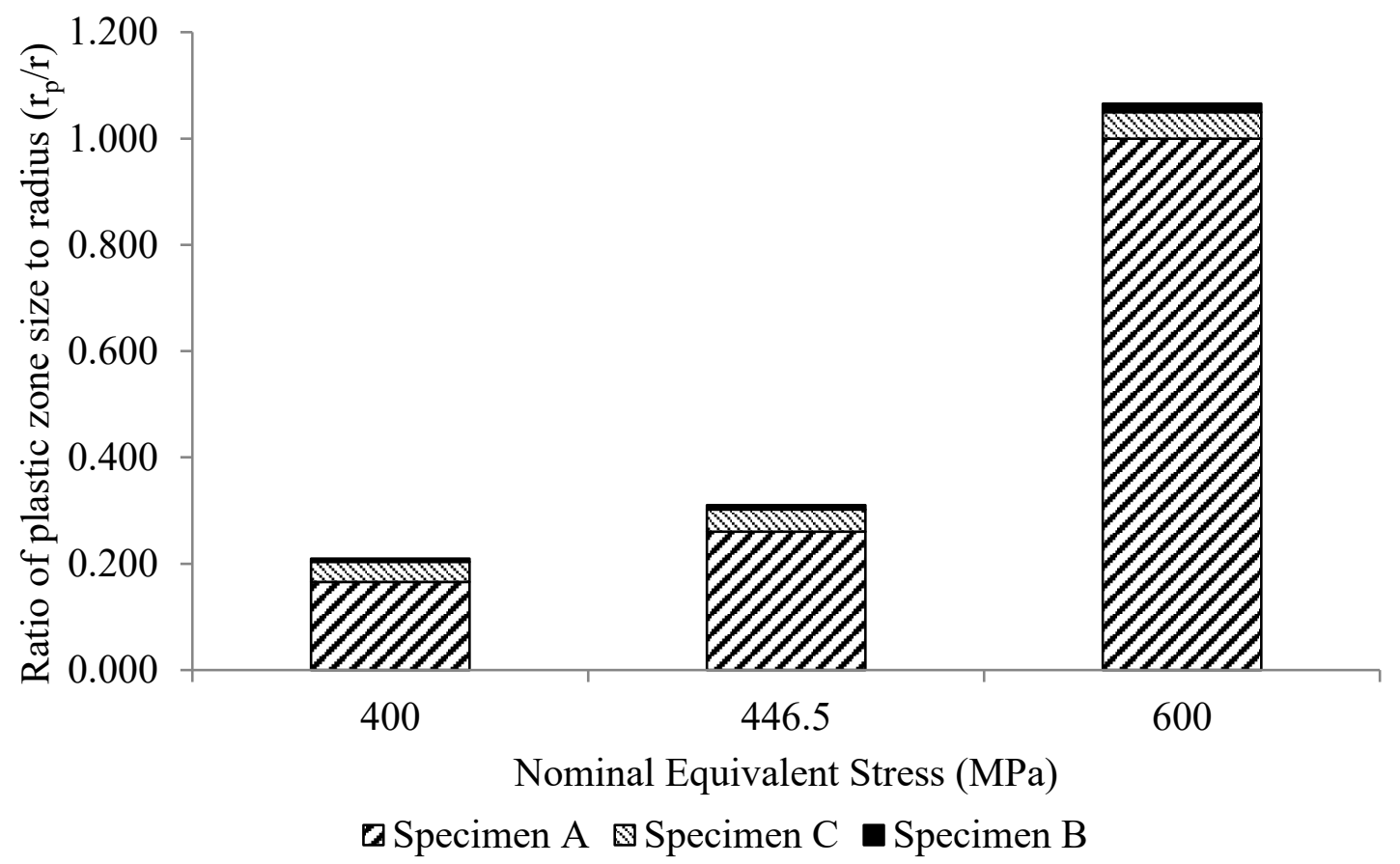

Figure 4-13: Ratio of plastic zone size to specimen groove radius for Test 1a (400 MPa), $1 \mathrm{~b}(446.5 \mathrm{MPa})$ and $1 \mathrm{c}(600 \mathrm{MPa})$ 


\subsection{Verification of Non-proportional Loading of Notched Specimens}

Two tests were performed to test the ability of the three implemented notch correction methods (ESED, Neuber, and Unified) and the stress-strain model in predicting nonproportional hardening; for these verification tests, experimental results from the literature were used as a benchmark. The first test is a basic box-patterned stress path shown in Figure 4-14 where the elastic notch normal stress and shear stress are reversed one at a time for a maximum nominal equivalent stress amplitude of $\frac{\Delta \sigma_{e q}}{2}=296 \mathrm{MPa}$. The second test is an unequal frequency stress path shown in Figure 4-15 where the tensile/compressive load is cycled three times per cycle of the shear load a maximum nominal equivalent stress amplitude of $\frac{\Delta \sigma_{e q}}{2}=566 \mathrm{MPa}$. The experimental strain data for both tests were obtained from Ince et al. (2014), and was originally reported by Barkey (1993). The experimental data is based on a SAE 1070 steel cylindrical specimen with geometry as shown in Figure 4-1 with $\mathrm{R}=25.4 \mathrm{~mm}$ and $\rho / \mathrm{t}=1$ and $\mathrm{R} / \mathrm{t}=2$. This results in stress concentration factors of $\mathrm{K}_{\mathrm{t}, \mathrm{ax}}=1.42$ and $\mathrm{K}_{\mathrm{t}, \mathrm{t}}=1.15$ (Ince, Glinka, \& Buczynski, 2014). Non-proportional loading verification tests with higher stress concentration factors could not be performed due to lack of experimental data from the literature.

For each test, the complete stress path was cycled 8 times to obtain a stable result. The material properties defined in Section 4.2 for SAE 1070 were used in addition to a manually inputted factor of non-proportionality of $\Phi=0.7$, following the value used by Ince et al. (2014). To achieve convergence in the final strain results, a maximum stress increment allowable of $20 \mathrm{MPa}$ was used for the stress-strain analysis. 


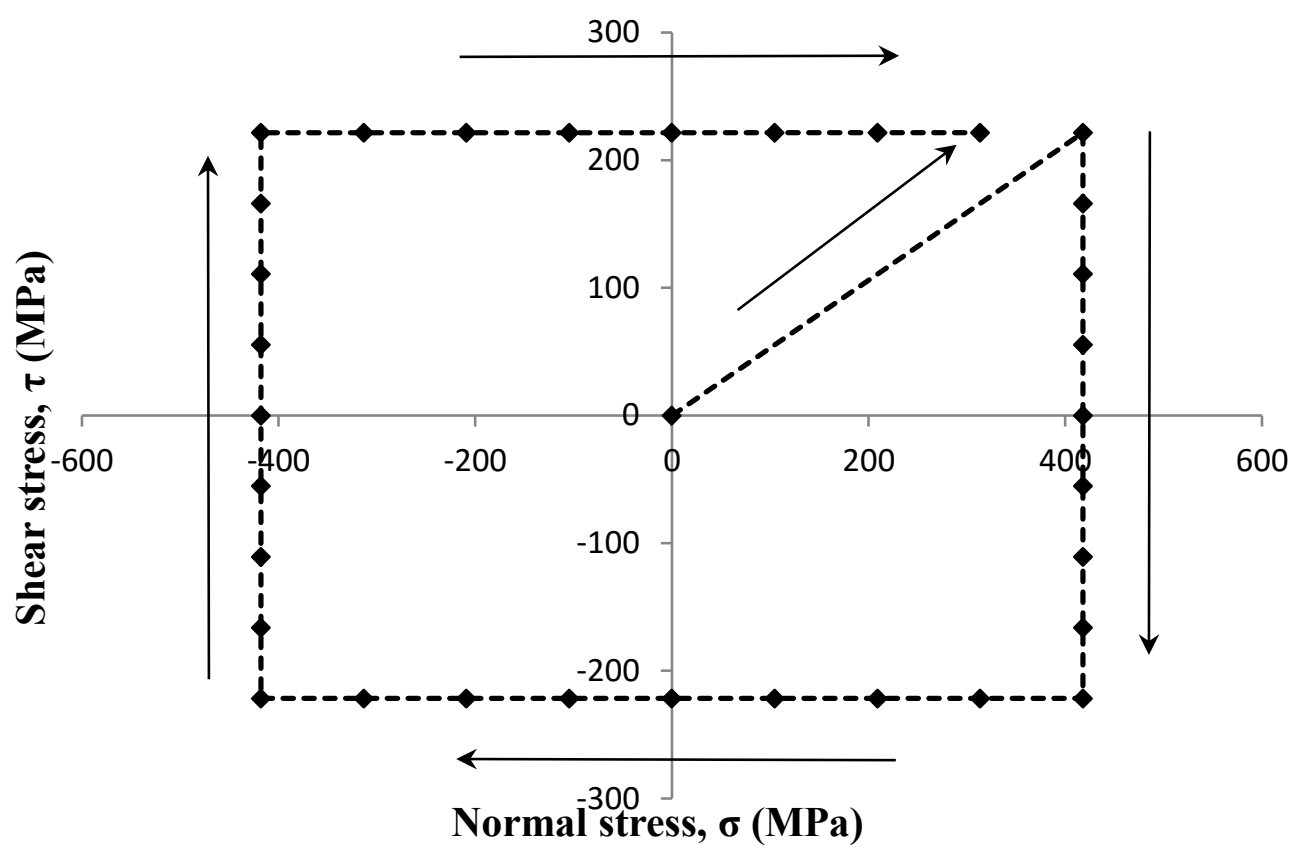

Figure 4-14: Box-patterned stress path used to test cyclic non-proportional loading.

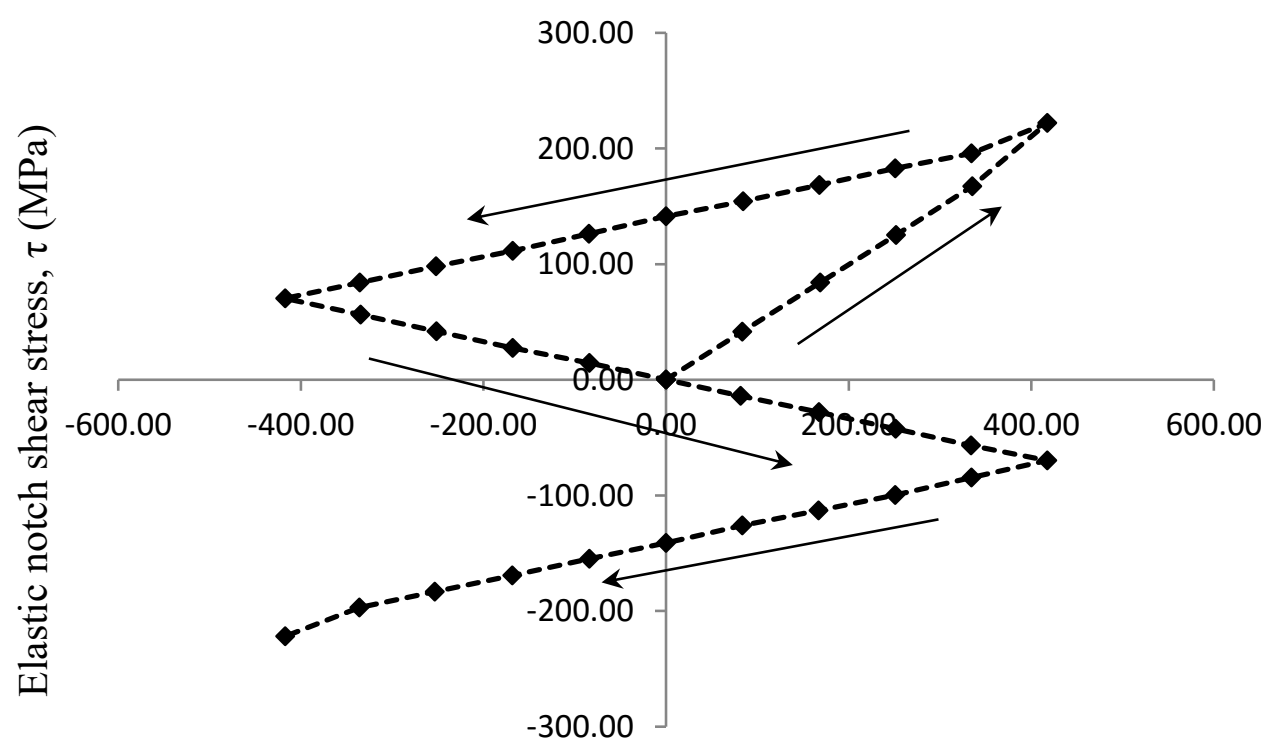

Elastic notch axial stress, $\sigma(\mathrm{MPa})$

Figure 4-15: Unequal frequency stress path used to test cyclic non-proportional loading. 


\subsection{Results and Discussion}

The results of the box-patterned stress path tests are presented in Figure 4-16 to Figure 4-18 for the three notch correction methods used. The strain predictions made by the ESED approach follow the stress path shape quite precisely, indicating an independence of axial and shear strain from one another. This is contrary to the experimental results in that in the events of plastic straining at the corners of the box pattern axial-shear strain coupling is expected. The axial-shear strain coupling is present in the Neuber and Unified analyses in Figure 4-17 and Figure 4-18, however the shear and axial strains appear to fall short in the positive direction.

Overall, there is a general under-prediction of the axial strain range of between 10 and $25 \%$, and an under-prediction of the shear strain range between 25 and $35 \%$. The best performing notch correction method was the multiaxial Neuber's rule which results in axial strain range under-predictions of $11 \%$ and $15 \%$ for the box-pattern and unequal frequency stress paths, respectively. The shear strain range predictions by the Neuber notch correction method is also slightly better than the other two methods with under-predictions of $15 \%$ and $28 \%$ for the box-pattern and unequal frequency stress paths, respectively. The under-prediction of the axial and shear strains are consistently worse for the unequal frequency stress path, which has an equivalent stress amplitude of roughly double that of the yield strength of SAE 1070. These under-predictions are to be expected based on the results of the proportional loading verification tests where for specimen A, which has comparable geometry to the specimens used by Ince et al. (2014) with respect to the notch, it was found that the shear stresses would see increasing under-predictions with increasing load.

Compared to results made by Ince et al. (2014) based on Garud's hardening model which reported under-predictions of 4-6\% for axial strain ranges and under-predictions of $11-15 \%$ for shear strain ranges this is a significant under-prediction, particularly in the shear strain 
range, that should be addressed in future iterations of stress-strain model. Furthermore, it was noticed that there was a slight offset in the normal strain in the experimental data that occurred prior to the initial loading sequence. It is not clear at the present time as to where this offset came from, though it is hypothesized that there could have been prior plastic strain accumulation in that one axial direction in earlier stages of the experiment that is not evident in the literature. 


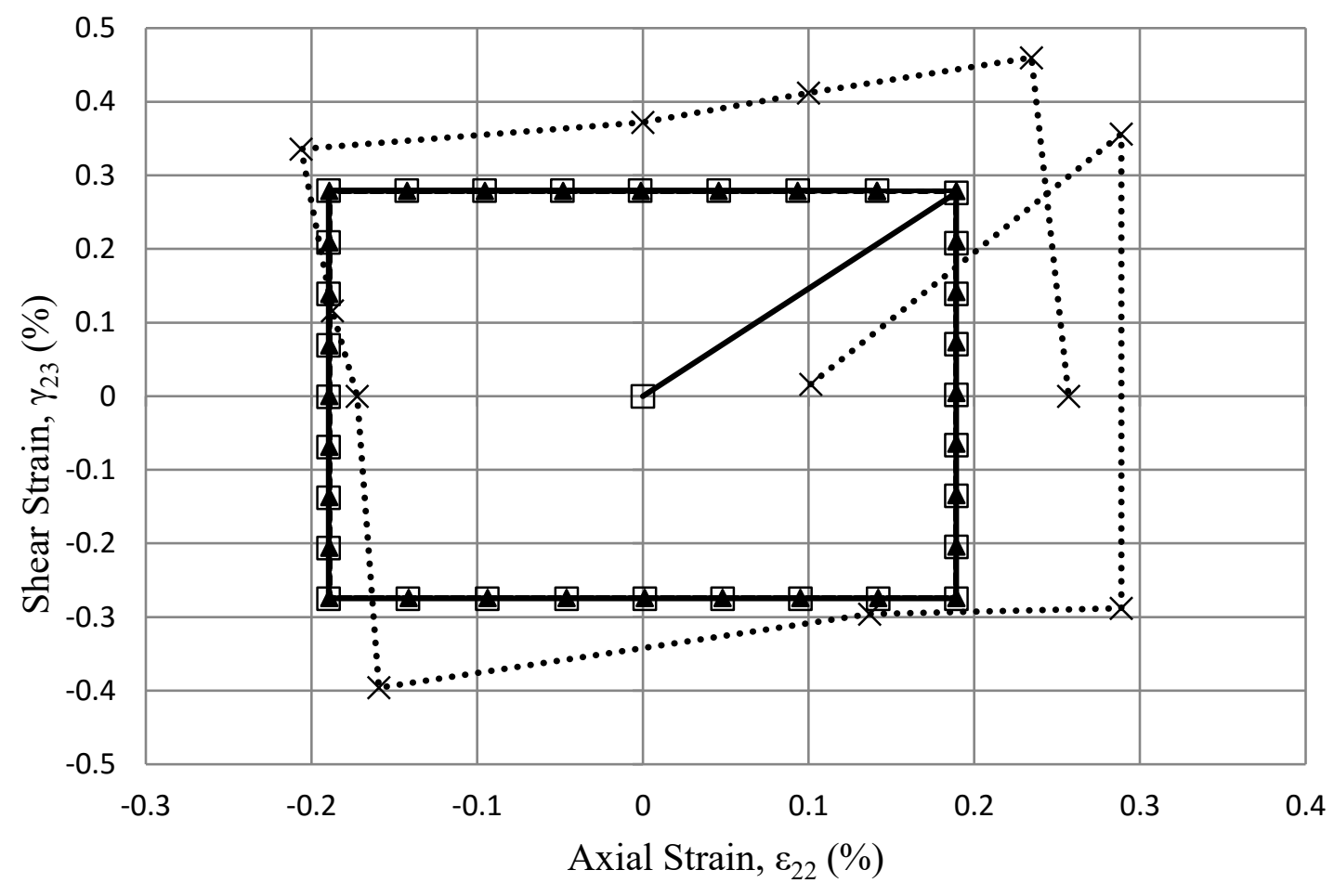

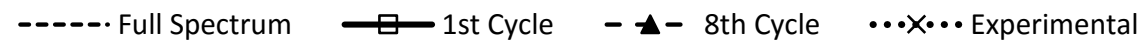

Figure 4-16: Strain predictions using incremental ESED notch correction for boxpatterned stress path compared to experimental data for SAE 1070 (Ince, Glinka, \& Buczynski, 2014). 


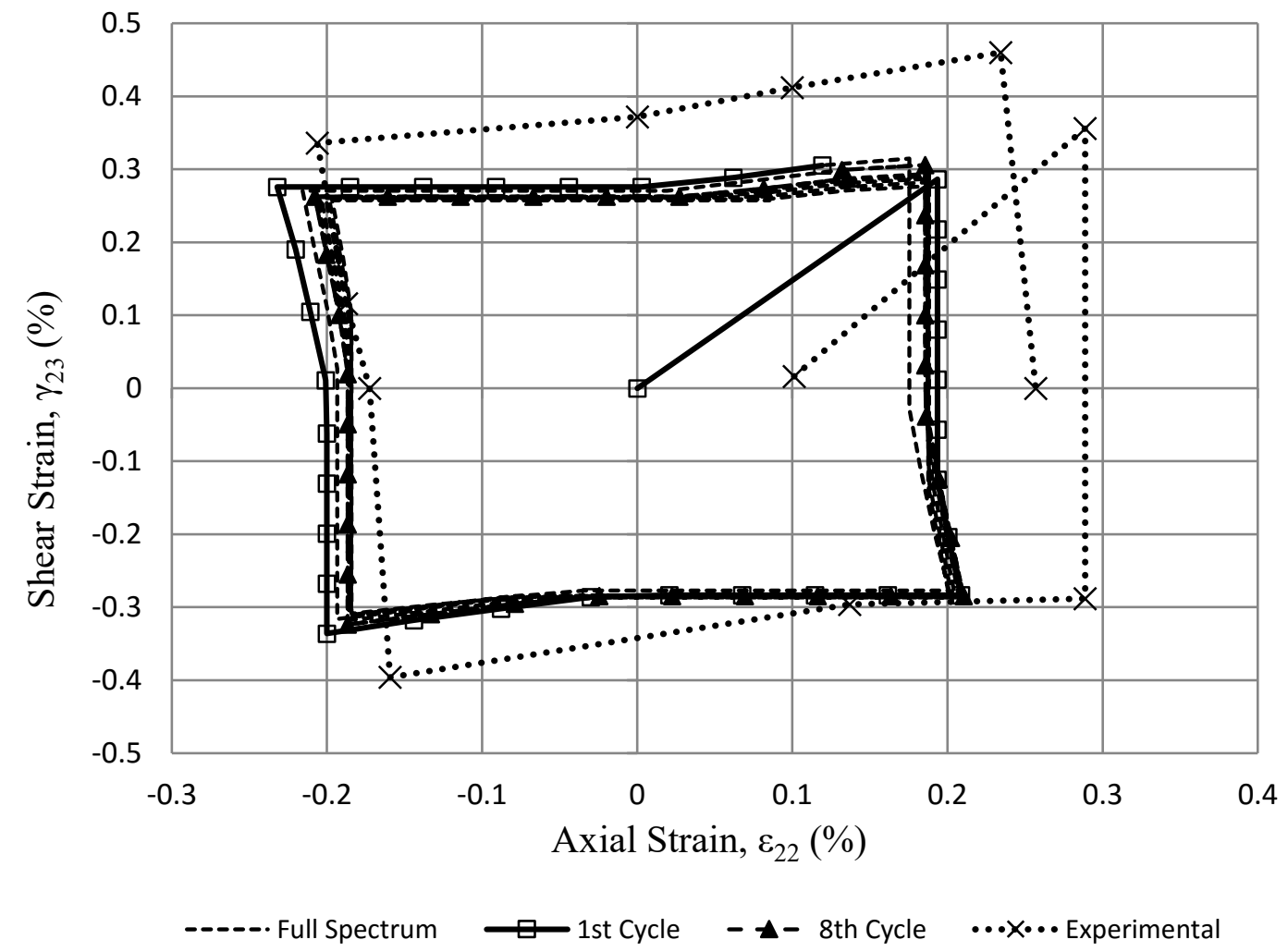

Figure 4-17: Strain predictions using the multiaxial Neuber's rule notch correction for box-patterned stress path compared to experimental data for SAE 1070 (Ince, Glinka, \& Buczynski, 2014). 


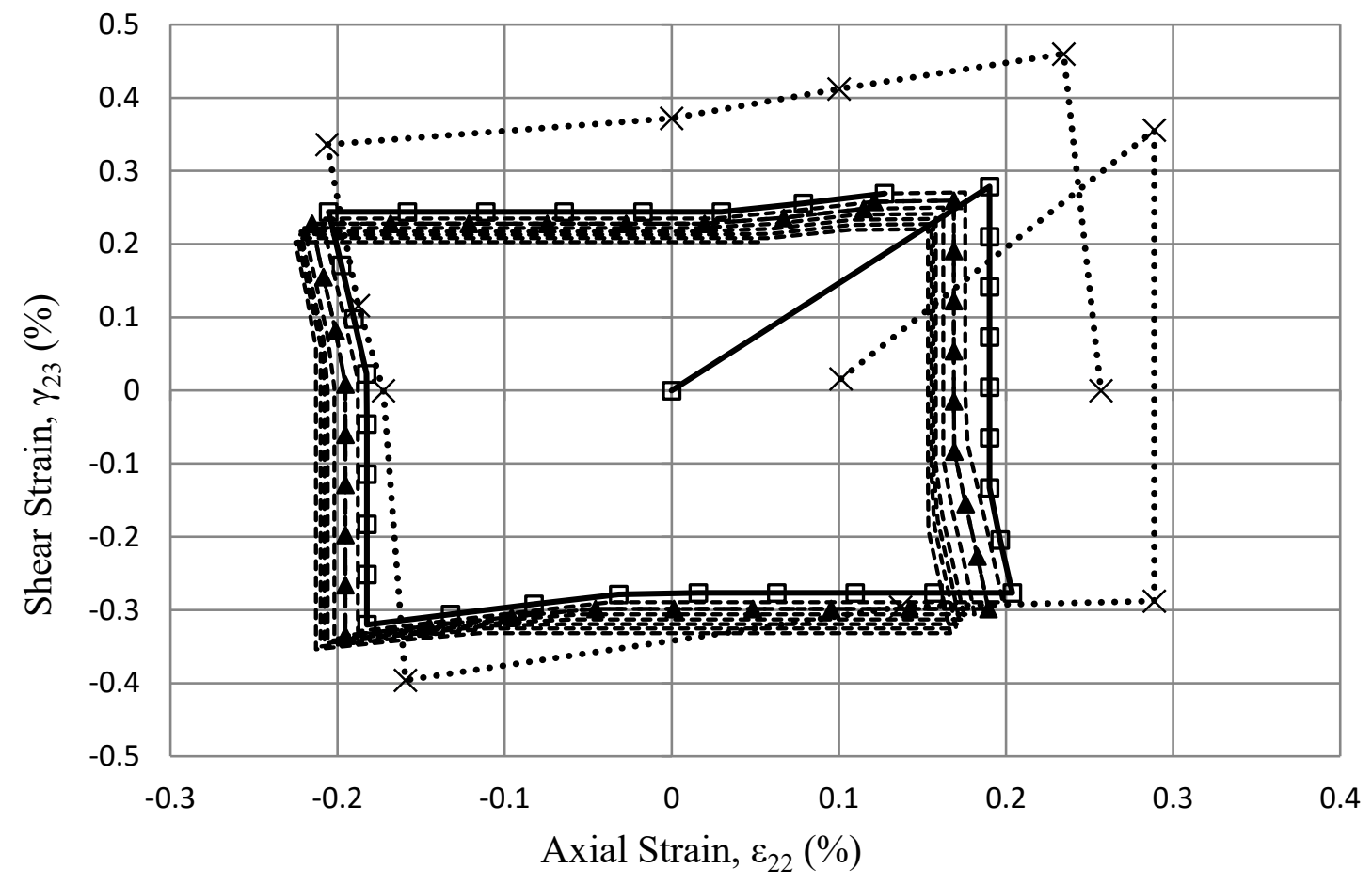

Full Spectrum $\quad \square$ 1st Cycle $\quad-\boldsymbol{\Delta}-$ 8th Cycle $\quad \cdots \times$.... Experimental

Figure 4-18: Strain predictions using Unified notch correction for box-patterned stress path compared to experimental data for SAE 1070 (Ince, Glinka, \& Buczynski, 2014). 


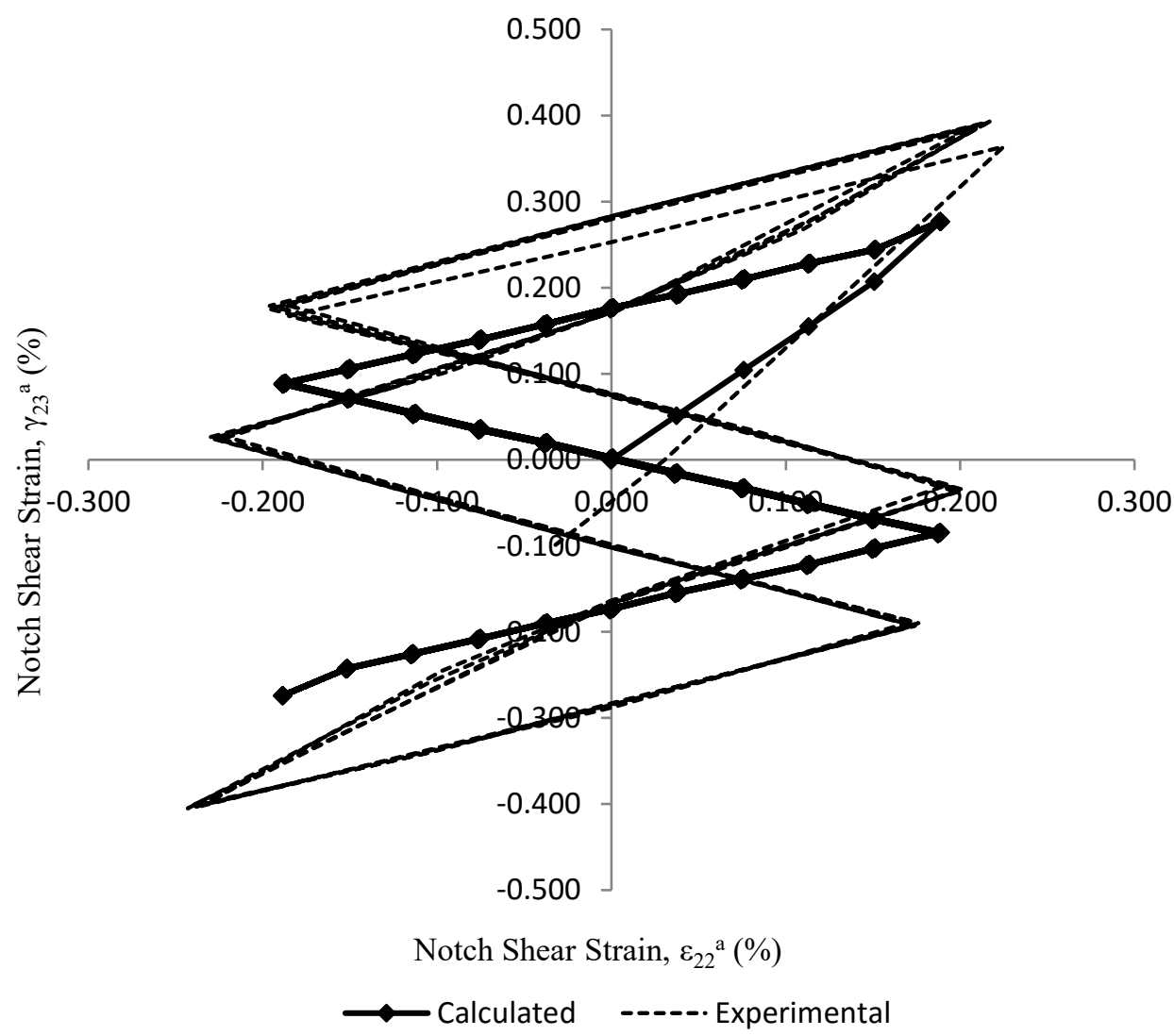

Figure 4-19: Strain predictions using ESED notch correction for unequal frequency stress path compared to experimental data for SAE 1070 (Ince, Glinka, \& Buczynski, 2014). 


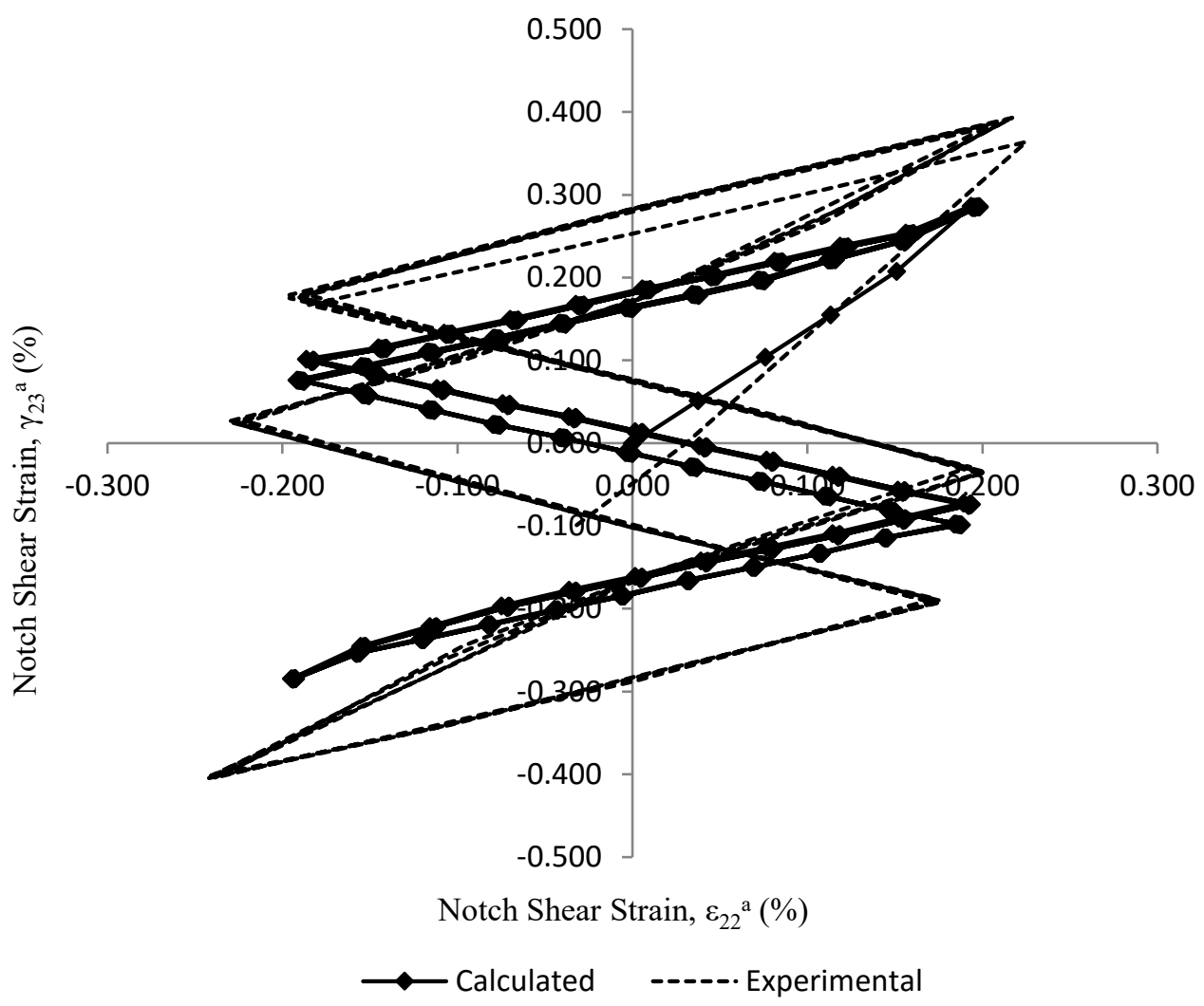

Figure 4-20: Strain predictions using Neuber notch correction for unequal frequency stress path compared to experimental data for SAE 1070 (Ince, Glinka, \& Buczynski, 2014). 


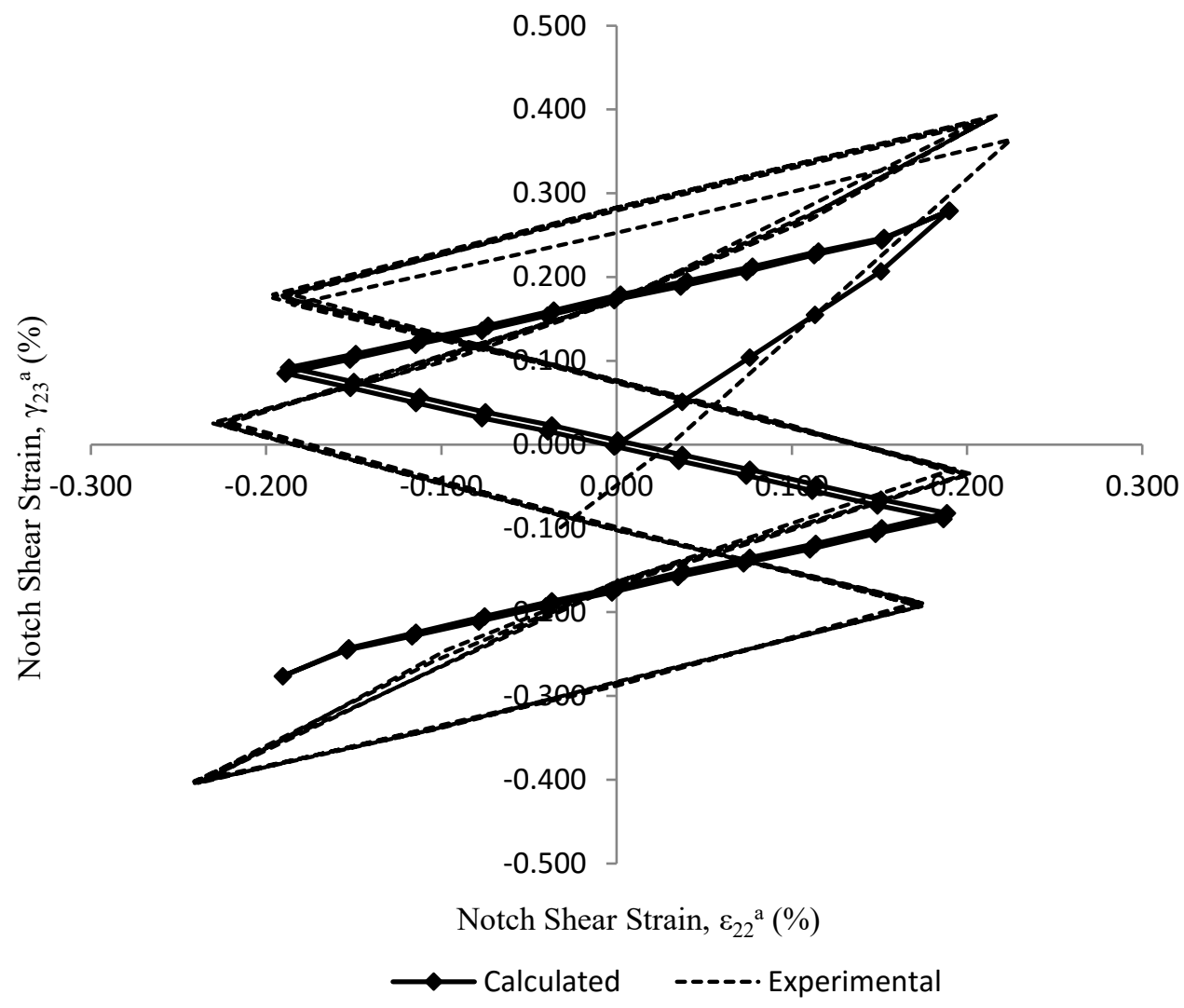

Figure 4-21: Strain predictions using Unified notch correction for unequal frequency stress path compared to experimental data for SAE 1070 (Ince, Glinka, \& Buczynski, 2014). 
Table 4-5: Strain range prediction comparisons for non-proportional loading verification tests.

\begin{tabular}{|c|c|c|c|c|}
\hline \multirow[b]{2}{*}{ Test } & \multirow{2}{*}{$\begin{array}{l}\text { Strain } \\
\text { Range }\end{array}$} & \multicolumn{3}{|c|}{$\begin{array}{c}\% \text { Difference with respect to } \\
\text { experimental data }\end{array}$} \\
\hline & & ESED & NEUBER & UNIFIED \\
\hline \multirow{2}{*}{$\begin{array}{r}\text { Box Pattern } \\
\left(\Delta \sigma_{e q} / 2=296 \mathrm{MPa}\right)\end{array}$} & $\Delta \varepsilon_{22}(\%)$ & -23.6 & -11.2 & -20.0 \\
\hline & $\Delta \gamma_{23}(\%)$ & -35.4 & -25.6 & -28.9 \\
\hline \multirow{2}{*}{$\begin{array}{l}\text { Unequal Frequency } \\
\left.\Delta \sigma_{e q} / 2=566 M P a\right)\end{array}$} & $\Delta \varepsilon_{22}(\%)$ & -17.1 & -14.7 & -16.7 \\
\hline & $\Delta \gamma_{23}(\%)$ & -30.6 & -28.2 & -30.1 \\
\hline
\end{tabular}




\subsection{Conclusions}

The incremental ESED, multiaxial Neuber's rule, and incremental Unified notch analysis methods were implemented in the stress-strain analysis program alongside the JiangSehitoglu kinematic hardening model discussed in Section 3.1. Verification tests were carried out to determine how well the implementation of the three different methods performed with proportional and non-proportional loading histories. Overall, the three methods performed similarly for both loading types.

For proportional loading histories, the predictions made by the three notch analysis methods were mostly found to be within $10-20 \%$ of finite element analysis predictions. The proportional loading histories were further used with three different notch specimens where the geometry and material were varied. It was found that the notch correction methods performed best where there is a high stress concentration and when the material is less ductile. For low stress concentration and high ductility, predictions made by the notch corrections made would tend to approach $20 \%$ or more error with respect to the finite element predictions due to the low constraint at the point of analysis.

For non-proportional loading histories, the notch analysis methods under-predicted experimental results found in literature by up to $30 \%$ in some cases. The multiaxial Neuber's rule produced the best results overall for both axial and shear strain range predictions, however it still under-predicted the experimental results significantly. The errors were largest in the unequal frequency tests, which had the largest amount of plastic deformation. Further noting that the specimens used in the literature were of low stress concentration factor, these observations reinforced the conclusion from the proportional loading verification tests that under-predictions of the axial and shear strain are to be expected where there is low geometric constraint. Caution should be applied in performing fatigue analyses using these methods in these situations as the predicted strains in regions of significant plasticity will most likely be non-conservative. In the future additional tests can be performed for specimens with higher stress concentration factors to provide a more complete understanding of the behaviour of the notch correction methods. 


\section{Chapter: Multiaxial Cycle Counting Procedures}

Cycle counting procedures are required as part of a fatigue analysis to simplify variable amplitude load histories into blocks of constant amplitude. Similar to the rainflow cycle counting procedure developed for uniaxial fatigue analysis, the Wang-Brown and Bannantine-Socie cycle counting procedures were developed to count multiaxial stress histories. The detailed steps for these two procedures were presented in Section 2.3.4. This chapter aims to provide insight into how the procedures were implemented and will compare the two procedures in terms of the stress ranges that they predict.

\subsection{Example of Wang-Brown Cycle Count}

The detailed steps for the Wang-Brown cycle counting procedure presented in Section 2.3.4 is illustrated in Figure 5-1. These steps are followed in order to establish the pairs of load cases that will form each reversal. This section presents a brief example of the execution of the Wang-Brown cycle count using sample axial and shear strain history from Wang \& Brown (1996) shown in Figure 5-2. The results of this example match the example presented in Wang \& Brown (1996) and were used as a reference to ensure that the implemented program would generate the expected results. The following discussion walks through the first two iterations of the cycle count.

Each load case shown in Figure 5-2 is a combined axial-torsion load. The axial and shear strains are then used to calculate the equivalent strain history shown in Figure 5-3. In this case, Load Case 1 (point $\mathrm{A}$ ) is the maximum turning point, so the first equivalent strain history can be calculated relative to this load case and is also shown in Figure 5-3. Note that the relative strains are calculated based on the components of strain and not on the values of equivalent strain. 


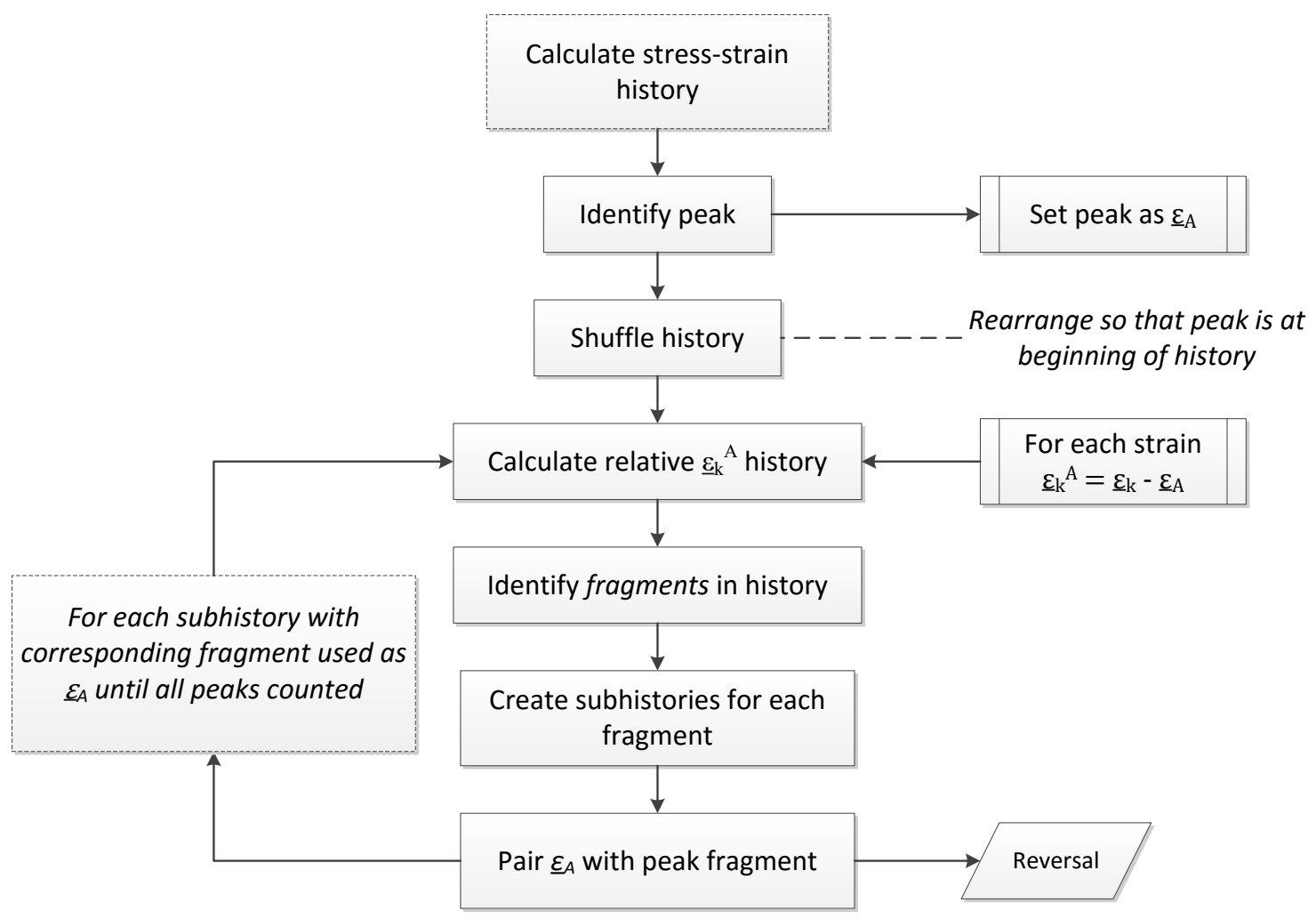

Figure 5-1: Illustration of algorithm used for the Wang-Brown cycle counting procedure.

With the equivalent relative strain history with respect to $\mathrm{A}$, two more turning points $\mathrm{B}$ and $\mathrm{D}$ (also referred to as fragments) are identified as shown in Figure 5-3. Load case D (load number 47) is the maximum turning point and is paired with the reference strain of the relative strain history, load case A (load number 1). This pair is identified as a reversal, i.e. half of a full cycle. The load cases from B to D and D to E, inclusively, form two sub-histories (or fragments) upon which two new relative equivalent strain histories are calculated with respect to the fragment points B and D as shown in Figure 5-4.

Since load case $\mathrm{H}$ in Figure 5-4 is the maximum turning point in its respective sub-history, a second reversal is formed by pairing load case 10 with load case 32. Two fragments are identified from this sub-history between load cases $\mathrm{G}$ and $\mathrm{H}$, and between load cases $\mathrm{H}$ and I. Two equivalent relative strain histories are further calculated relative to load cases $\mathrm{G}$ and $\mathrm{H}$ and are shown in Figure 5-5. From the D' sub-history, a reversal is simply identified between load case D' and load case J. 
From the $\mathrm{G}^{\prime}$ relative strain history, load case $\mathrm{M}$ is identified as a fragment point and a reversal is identified between load case G' and the maximum load case N. From the H' relative strain history, load case $\mathrm{O}$ is identified as a fragment and a reversal is identified between load case $\mathrm{H}^{\prime}$ and load case P. Continuing to iterate until all load cases are included within a reversal will result in the pairs summarized in Table 5-1.

Table 5-1:Load case pairs determined using the Wang-Brown cycle counting procedure on the sample axial-torsion strain data from Wang \& Brown (1996).

\begin{tabular}{|c|c|}
\hline Load Case 1 & Load Case 2 \\
\hline 1 & 47 \\
\hline 11 & 32 \\
\hline 47 & 57 \\
\hline 21 & 32 \\
\hline 32 & 47 \\
\hline 25 & 32 \\
\hline 39 & 47 \\
\hline
\end{tabular}




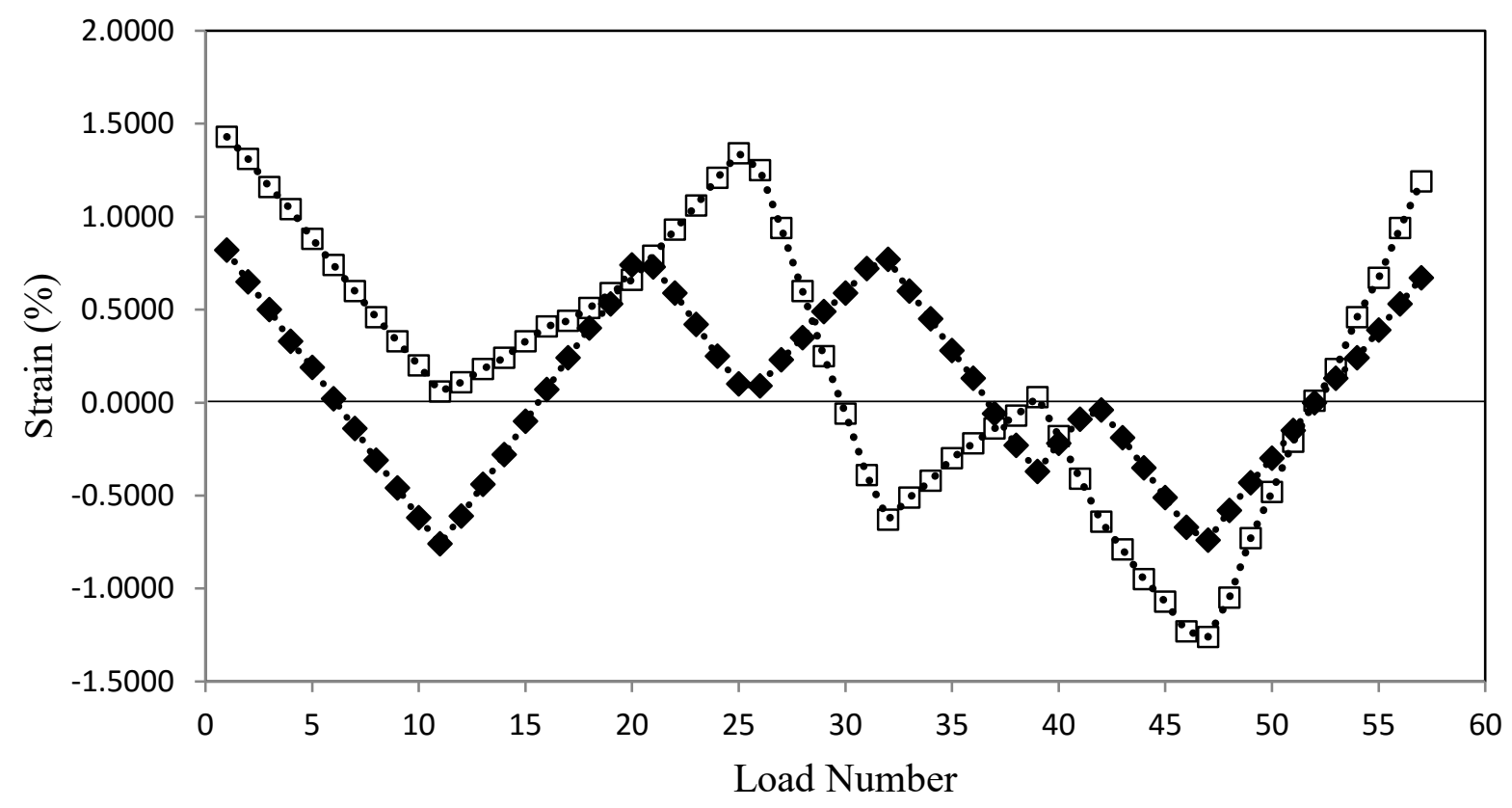

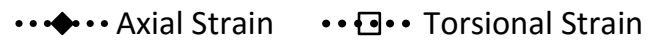

Figure 5-2: Sample strain data used to test multiaxial cycle counting procedures (Wang \& Brown, 1996). 


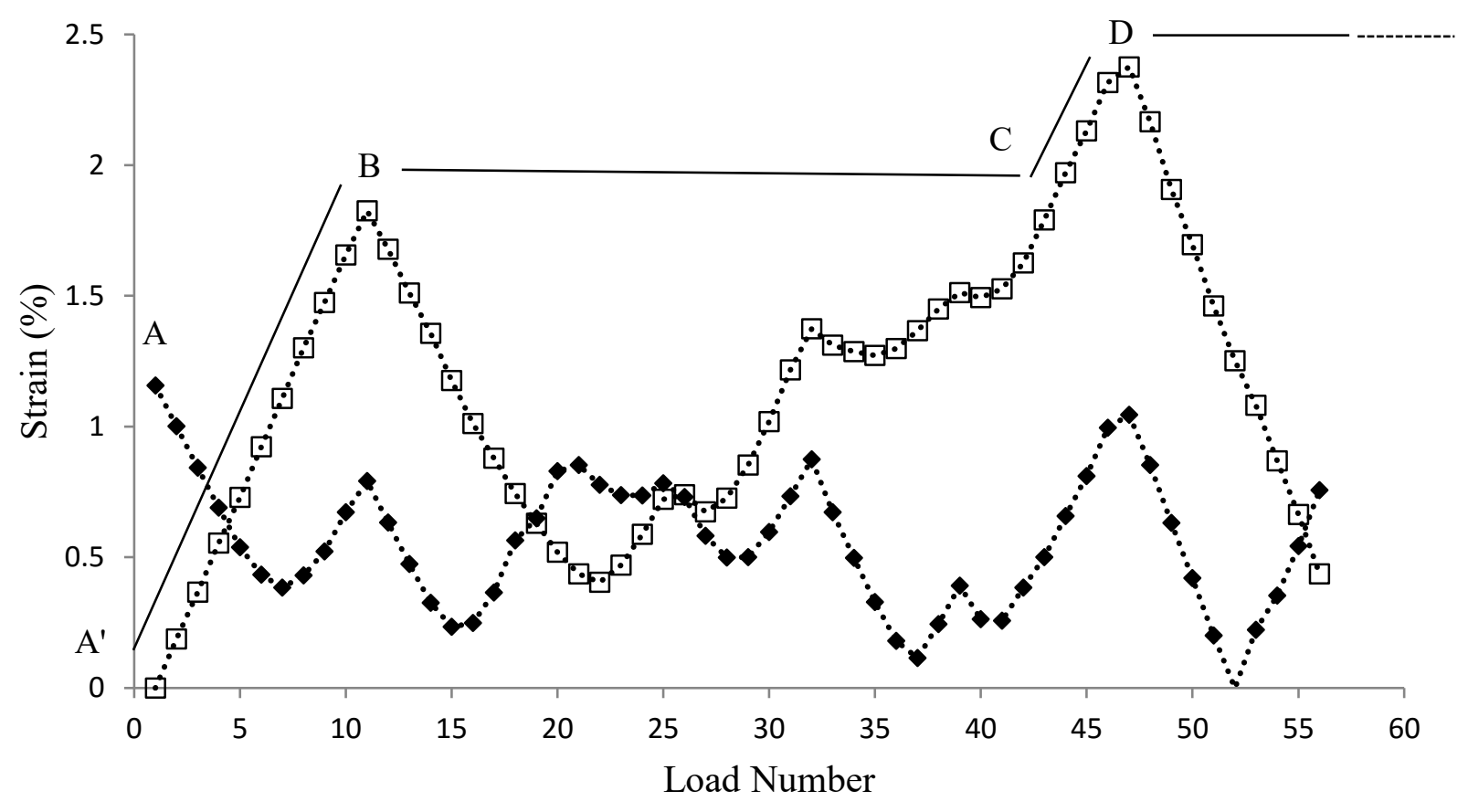

…. Eq. Strain $\quad \cdots \circledast \cdots$ Relative Eq. Strain A

Figure 5-3: Equivalent strain history and equivalent relative strain history with respect to point A calculated in the first iteration of cycle count. 


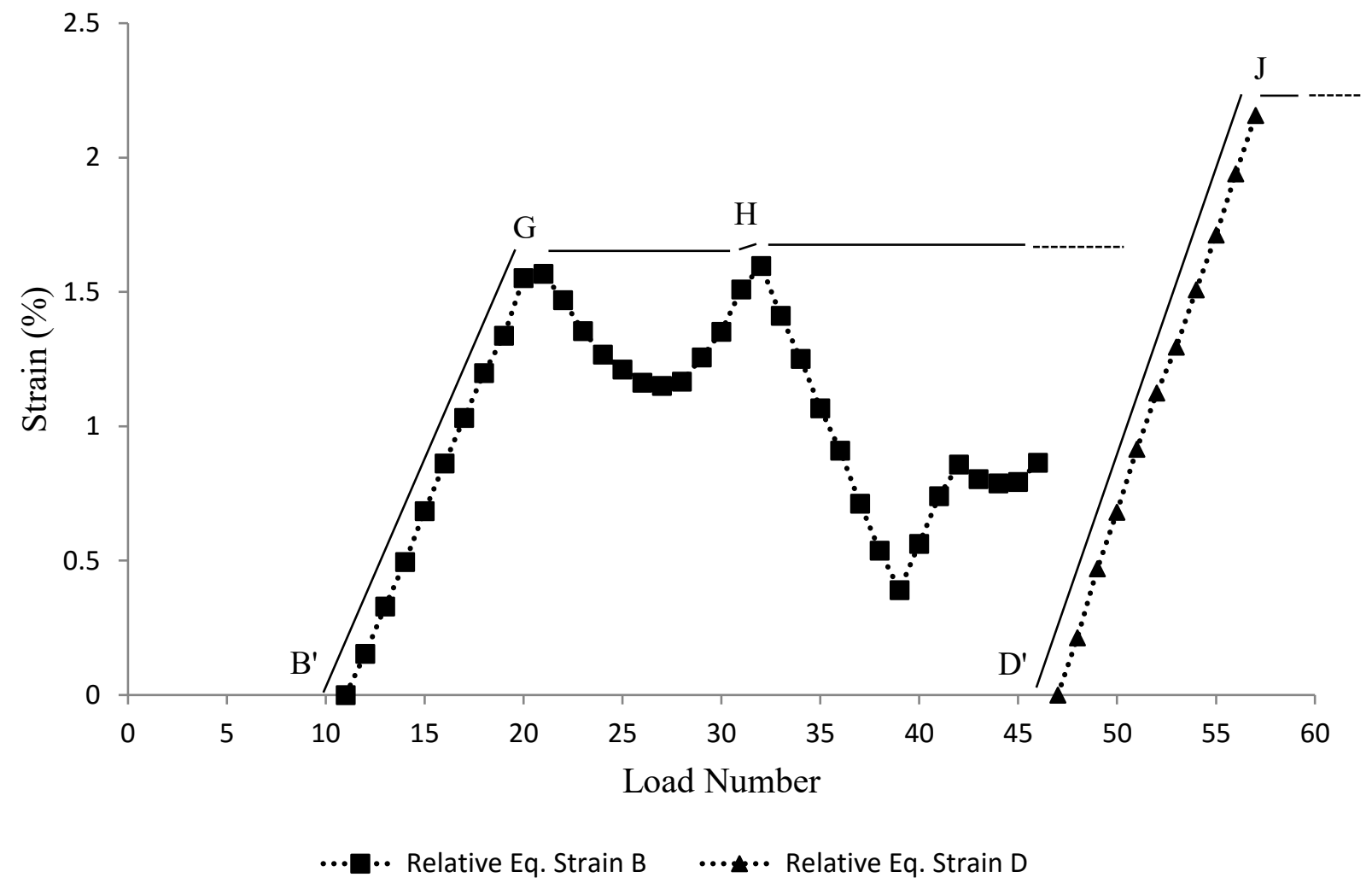

Figure 5-4: Equivalent relative strain histories with respect to point $\mathrm{B}$ and $\mathrm{D}$ calculated in the second iteration of cycle count. 


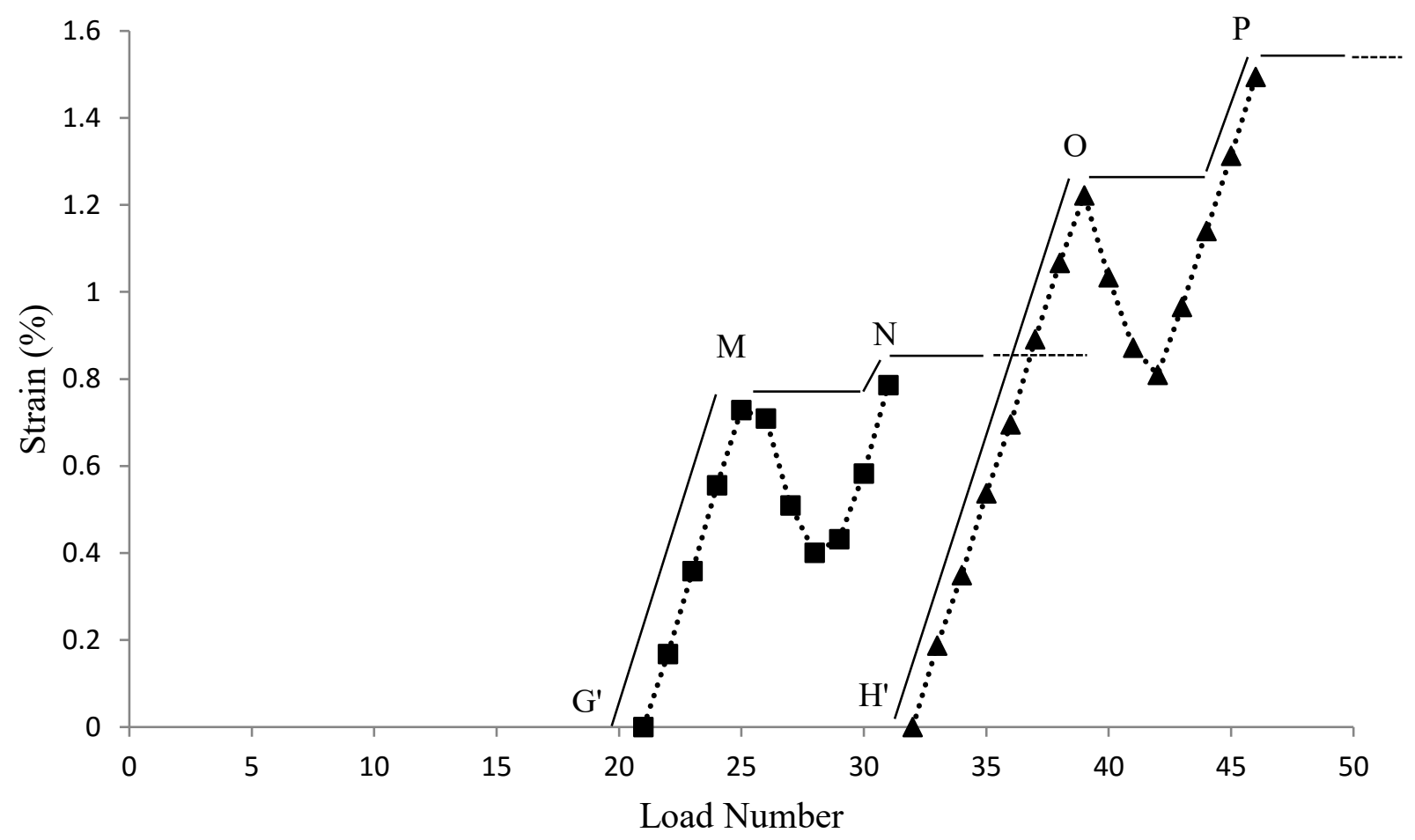

$\cdots \square \cdot \cdots$ Relative Eq. Strain G $\quad \cdots \wedge \cdots$ Relative Eq. Strain H

Figure 5-5: Equivalent relative strain histories with respect to point $\mathrm{G}$ and $\mathrm{H}$ calculated in the third iteration of cycle count. 


\subsection{Implementation of the Bannantine-Socie Cycle Counting Procedure}

The Bannantine-Socie cycle counting procedure uses the standard rainflow cycle counting algorithm defined in ASTM E1049-85 as described in Section 2.2.4. With the rainflow cycle count, the Bannantine-Socie procedure will identify both reversals and full cycles, depending on the relative magnitudes of the peaks and valleys.

A work flow diagram illustrating the procedure for running a Bannantine-Socie cycle count is shown in Figure 5-6. For each critical plane designated by the user, a rainflow cycle count is performed based on the reference strain component as if it were a uniaxial analysis. When performed with a fatigue analysis, the fatigue damage is calculated for each critical plane cycle count and the maximum value is used.

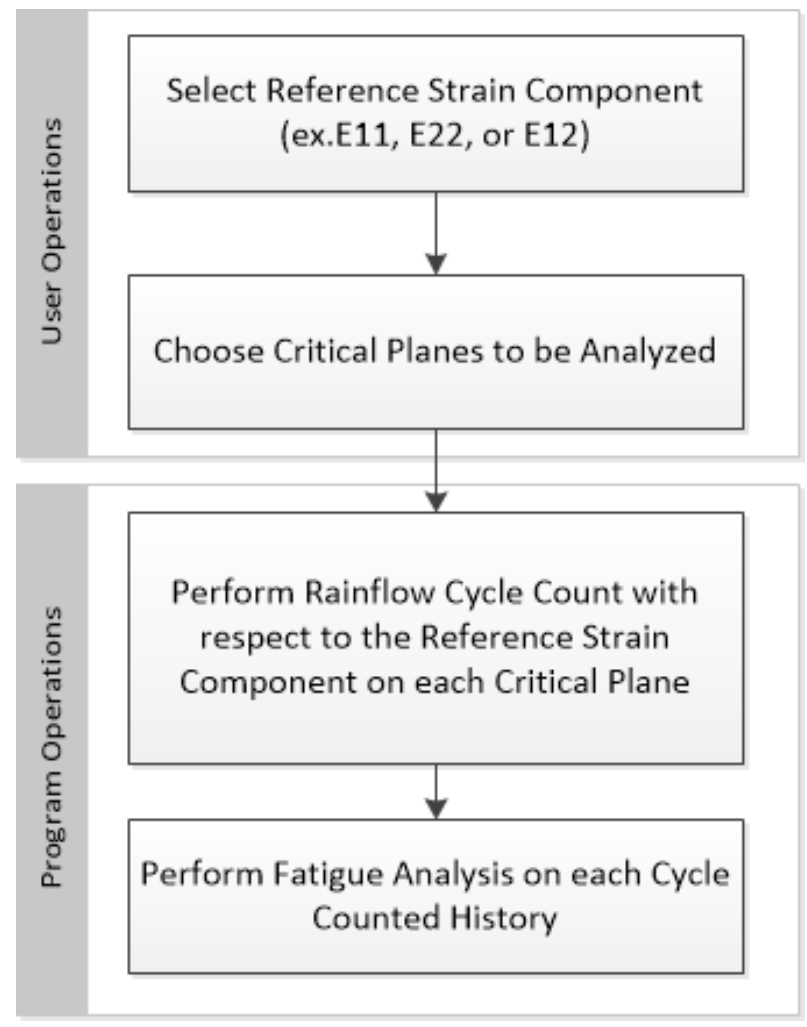

Figure 5-6: User controls for Bannantine-Socie cycle counting procedure. 


\subsection{Comparison of Wang-Brown and Bannantine-Socie Counting Procedures}

Using the sample strain history given in Figure 5-2, the cycle counted pairs formed by the WangBrown and Bannantine-Socie procedures were compared. The Bannantine -Socie procedures were run for $0^{\circ}, 45^{\circ}$, and $90^{\circ}$ critical angles with reference to the E22 strain. A histogram is presented in Figure 5-7 and Figure 5-8 to show the distribution of the axial and shear stress range, respectively, of the cycles counted using the Wang-Brown and Bannantine-Socie procedures. It is first noted that the Bannantine-Socie cycle counts all result in more reversals being counted than the Wang-Brown procedure. This difference occurs because the rainflow cycle count used by the Bannantine-Socie procedure includes a step that attempts to count full cycles (two reversals), whereas the Wang-Brown procedure only counts individual reversals.

Both cycle counting procedures are observed to predict the larger axial and shear stress ranges adequately. For the Bannantine-Socie procedures, the critical angles that result in the largest axial or shear stress range pair $\left(0^{\circ}\right.$ and $\left.90^{\circ}\right)$ also ends up being the cycle count that is most evenly distributed. This is in contrast to the cycle count performed on the $45^{\circ}$ critical plane, which results in cycles that are skewed towards the lower axial and shear stress ranges. The Wang-Brown cycle count appears to have an even stress range distribution while predicting the max stress range well.

\subsection{Modification of Wang-Brown Cycle Counting Procedure}

A significant difference between the Wang-Brown and Bannantine-Socie procedures is the identification of fully cycles within the rainflow algorithm of the Bannantine-Socie procedure. Since the rainflow algorithm has gained such significant acceptance from the engineering community for uniaxial fatigue analyses, it was deemed worthwhile to attempt to modify the Wang-Brown procedure to include the evaluation of full cycles. The following rules based on the rainflow counting procedure are proposed and adopted into a modified version of the Wang-Brown procedure:

1. If the sub-history being analyzed is the final sub-history in the relative strain history being analyzed, then the starting point (defined as point $\mathrm{S}$ ) is the first load case in that sub-history. 
2. If the starting point of a sub-history is $\mathrm{S}$, then the peak of this sub-history (once identified) will become the next starting point $\mathrm{S}$.

3. If a counted range does not contain the starting point $\mathrm{S}$, then this range will be considered a full cycle.

If a counted range contains the starting point $\mathrm{S}$, then this range will be a reversal, or half cycle. By using this modified version of the Wang-Brown procedure, identical results to the Bannantine-Socie procedure and standard rainflow count are produced for a uniaxial stress or strain history. While this does not represent an improved Wang-Brown procedure, it provides a third option for evaluation when considering the integration of the cycle counting procedures into the overall fatigue analysis process. These three cycle counting procedures (Wang-Brown, Bannantine-Socie, and modified Wang-Brown) will be compared further in Chapter 6 for their effect on the predicted fatigue life of the landing gear fuse pin. 


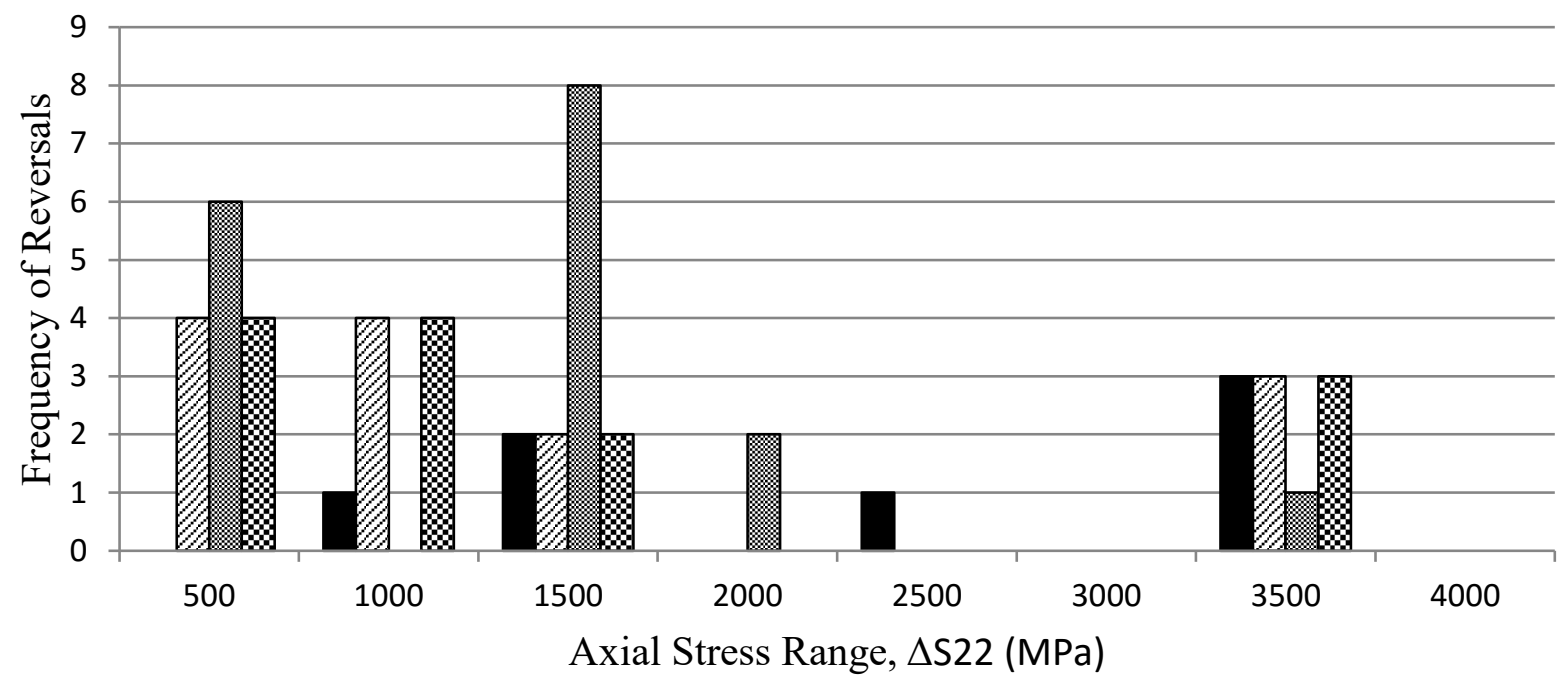

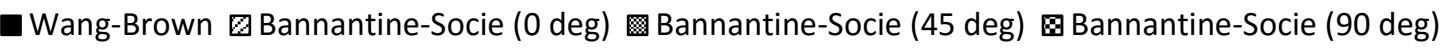

Figure 5-7: Axial stress range of cycles counted by Wang-Brown and Bannantine-Socie counting procedures. 


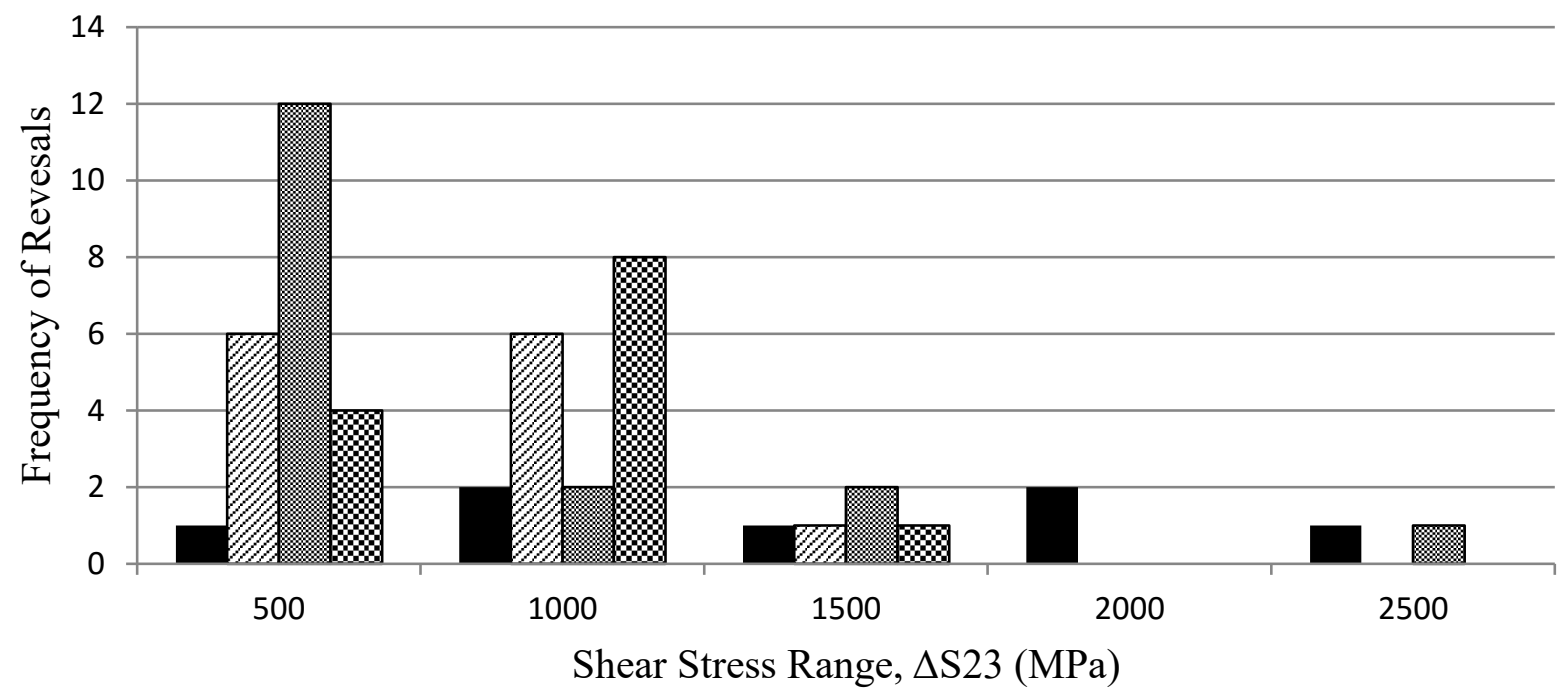

- Wang-Brown $₫$ Bannantine-Socie (0 deg) Bannantine-Socie (45 deg) $₫$ Bannantine-Socie (90 deg)

Figure 5-8: Shear stress range of cycles counted by Wang-Brown and Bannantine-Socie counting procedures 


\section{Chapter: Fatigue Analysis of Test Fuse Pins}

This chapter presents the preliminary and final results of the fatigue analysis of three fuse pin designs using the multiaxial fatigue methodologies presented in the previous chapters. Combinations of fatigue damage criterion, notch correction methods, and cycle counting procedures are tested in the analysis of each of the three pins to determine which best predicts the fatigue damage observed in test. The uniaxial fatigue analysis results are presented as well to establish a benchmark for improvements in the life prediction of the fuse pins.

\subsection{Test Fuse Pins}

UTC Aerospace Systems - Landing Gear Systems (UTAS-LS) has provided test data for three different fuse pins -each representing one of the three joints discussed in Section 2.4.1. The three fuse pins have all undergone complete assembly-level fatigue testing with one of the pins failing prematurely. All three of the test fuse pins are made out of a heat-treated low-alloy stainless steel. The details regarding the test fuse pins are summarized in Table 6-1. The detailed geometry of each test fuse pin was provided by UTAS-LS in the form of finite element assembly models in ABAQUS ${ }^{1}$.

Table 6-1: Summary fuse pins provided by UTAS-LS

\begin{tabular}{l|l|l|l} 
& Joint Type & Fatigue Failure in Test & Reference Figure \\
\hline Pin A & Clevis-Bushing & Yes & Figure 2-8 (p.43) \\
Pin B & Cantilever-Bushing & No & Figure 2-9( p.44) \\
Pin C & Cantilever-Spherical Bearing & No & Figure 2-10 (p.45)
\end{tabular}

${ }^{1}$ Due to the proprietary nature of the data provided by UTAS-LS, details such as material properties, fuse pin geometry, and loads cannot be provided in this thesis. 


\subsection{Stress Distribution in the Fuse Groove}

Static FEA using elastic and elastic-plastic material properties were performed by UTAS-LS for the three test fuse pins in ABAQUS and the results were provided for the fatigue analysis of the fuse groove of each pin. The FE models are all quarter-symmetric assembly models containing all relevant parts in the fuse pin joint similar to the illustrations in Figure 2-8, Figure 2-9, and Figure 2-10. As given in Table 6-1, Pin A is the clevis joint with bushings, Pin B is the cantilever joint with bushings, and Pin $\mathrm{C}$ is the cantilever joint with a spherical bearing and a bushing. Quadratic quadrilateral elements were used throughout each fuse pin with a refined mesh along the fuse groove. A maximum tension and a maximum compression analysis were performed for each fuse pin for a pure elastic analysis and an elastic-plastic analysis. The purpose of the pure elastic analysis is to determine the theoretical elastic notch stresses for each pin under tension and compression, while the elastic-plastic analysis is to be used for verification of the notch stresses and strains as predicted by the multiaxial stress-strain model for the maximum tension and compression load cases.

While the fuse pin joint is loaded in simple tension and compression, the type of joint assembly has a significant effect on the stress distribution within the fuse groove. As was illustrated in Figure 2-11, there are three active components in the fuse groove: the hoop stress, the longitudinal stress, and the shear stress. To illustrate the different stress states along the fuse groove for the three different joints, the three stress components at the fuse groove for a maximum compression load are plotted for each fuse pin in Figure 6-1, Figure 6-2, and Figure 6-3, respectively. The figure shows the stress distribution along the fuse groove at five different circumferential locations in the fuse groove. All of the stresses are normalized with respect to the maximum pure shear stress $\tau_{\max }$ that is calculated for each pin and the longitudinal positions have been normalized with respect to the width of the fuse groove for each pin. The normalization of these parameters removes the dependency of the stress distribution on the applied load and pin geometry.

Depending on the longitudinal location along the fuse groove, the stress state will vary due to the load path from the airframe and landing gear lugs into the fuse pins. In Figure 6-1, Figure 6-2, and Figure 6-3, the positive $\hat{z}$ is defined as the airframe side of the pin, while the negative $z$ is 
landing gear side of the pin. For positive $\hat{z}$, the first positive angular locations $\left(0^{\circ}, 45^{\circ}\right.$, and $90^{\circ}$ from the neutral axis) are located on the loaded side of the pin for a compressive load. The remaining two locations $\left(-45^{\circ}\right.$ and $-90^{\circ}$ from the neutral axis) are on the unloaded side of the pin; that is, these locations lie on the side of the pin with no direct compressive loads on the exterior of the pin from the airframe lug. For negative $\hat{z}$, the reverse is then true for each angular location.

Considering first the hoop stress distributions in Figure 6-1(a) to (c), it can be seen that the hoop stress overall becomes increasingly negative from Pin A to C. This correlates with a decreasing amount of constraint within the pin joint; Pin A is the most highly constrained due to the clevis configuration compared to the cantilever configuration of Pin $\mathrm{B}$ and $\mathrm{C}$, and the spherical bearing of the Pin $\mathrm{C}$ configuration further reduces the constraint of the joint. The opposite trend is seen in Figure 6-1(d) and (e), where Pin C has a more positive hoop stress and is tensile in some locations along the fuse groove. Since compressive stresses will tend to increase fatigue lives in general, this difference in the hoop stress distribution, which is predominantly compressive, can impact the fatigue life predictions significantly.

Figure 6-2 (a) for the longitudinal stress distributions at the neutral axis of the pin shows the impact of the spherical bearing in Pin C. The longitudinal stress distribution deviates significantly from the bushing-styled joints of Pin A and B, generally resulting in a longitudinal stress of the opposite sign. Away from the neutral axis, Figure 6-2 (b) and (c) show a similarity between the two cantilever joints of Pin B and C, with a slight deviation from the clevis joint of Pin A. Overall, these plots indicate clear longitudinal stress peaks away from the centre of the fuse groove which is significant since strain gauges during fatigue and static tests are typically placed at the centre of the fuse groove due to practical limitations.

The shear stress distributions at the neutral axis plotted in Figure 6-3 (a) shows increasing shear stress with decreasing constraint; i.e. Pin $\mathrm{C}$ has the maximum shear stress followed by Pin $\mathrm{B}$ and A, respectively. Furthermore, as observed in Figure 6-3 (b) to (e), the peak shear stress tends to shift towards the unloaded side of the fuse pin for the cantilever joints of Pin $\mathrm{B}$ and $\mathrm{C}$ while maintaining the trend that Pin $\mathrm{C}$ shows the highest normalized shear stress. For example, at the $45^{\circ}$ location in Figure 6-3 (b) the peak shear stresses for Pin B and C occur at a negative $\hat{z}$ position, 
where there is no direct load from the lug to the pin on that half of the pin. The reverse is then seen at the $-45^{\circ}$ location in Figure 6-3 (d) where the peak shear stress occurs at a positive ẑ position.

Understanding the stress distribution along the fuse groove for the three different pins is crucial to the developing a proper fatigue analysis procedure. From these distributions, it is observed that when doing a fatigue analysis positions representing the positive $\hat{z}$, the negative $\hat{z}$ and the centre of the fuse pin $(\hat{z}=0)$ need to be analyzed so as to account for both the longitudinal and shear stress peaks. The hoop stresses are also found to vary significantly with the three different pins and is expected to play an important role in determining the fatigue life of each pin. 

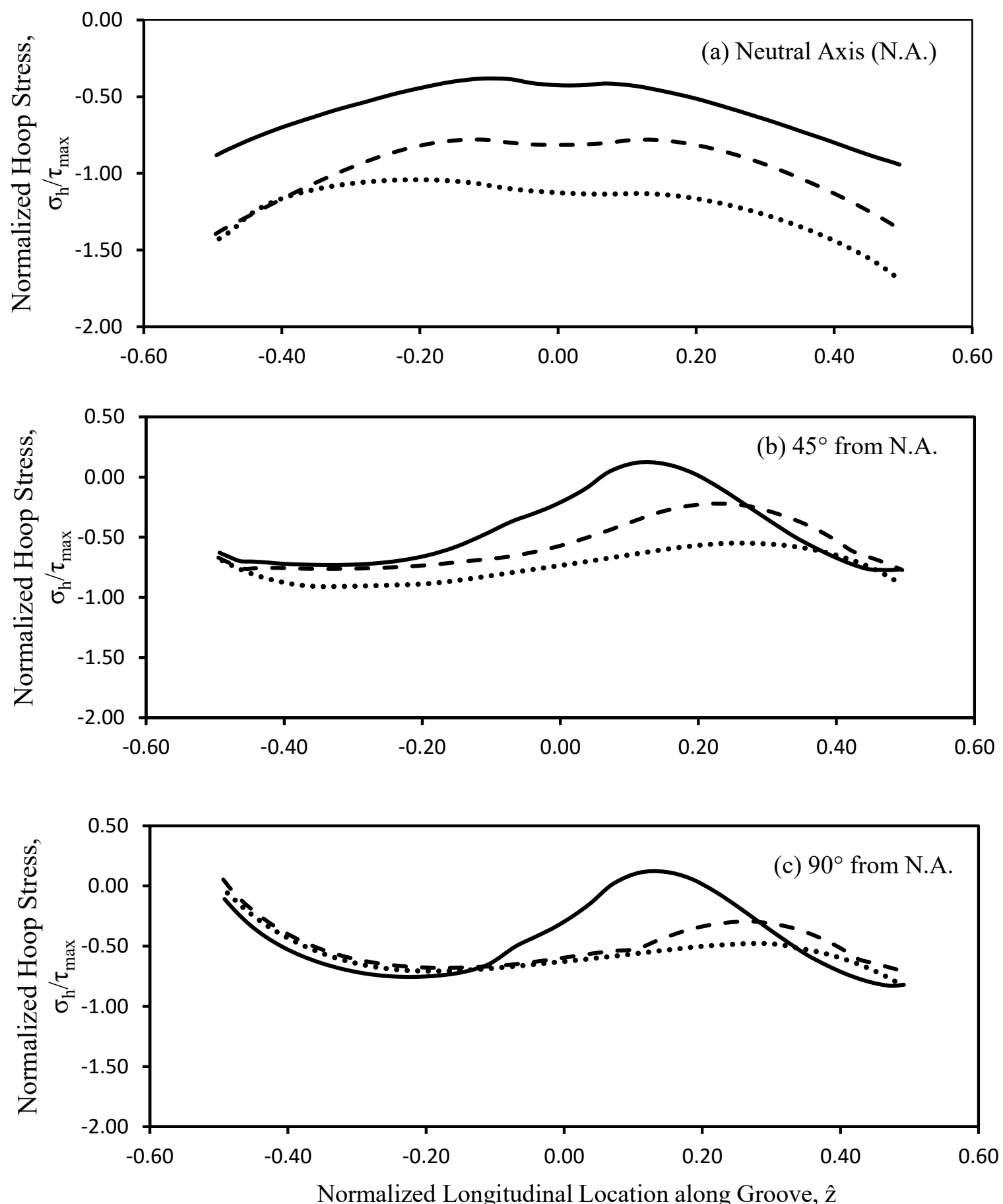

$\longrightarrow$ Pin A (Clevis+Bushing) - - Pin B (Cantilever+Bushing) -.... Pin C (Cantilever+Spherical)

Figure 6-1: Hoop stress distribution along fuse pin groove at (a) neutral axis; (b) $45^{\circ}$ from neutral axis; (c) $90^{\circ}$ from neutral axis; (d) $-45^{\circ}$ from neutral axis; and (e) $-90^{\circ}$ from neutral axis. Stresses determined from elastic-plastic FEA. 

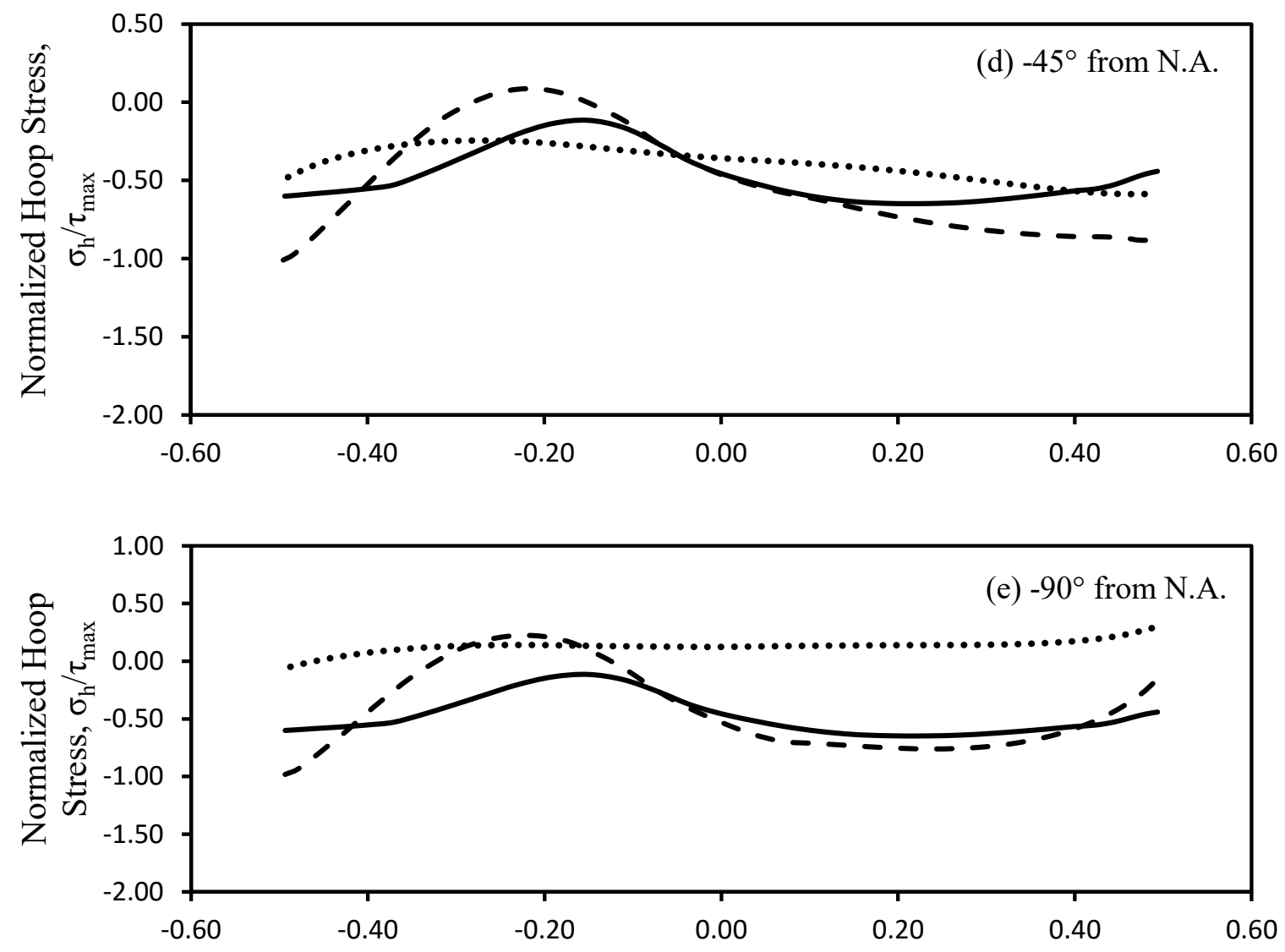

Figure 6-1 (continued): Hoop stress distribution along fuse pin groove at (a) neutral axis; (b) $45^{\circ}$ from neutral axis; (c) $90^{\circ}$ from neutral axis; (d) $-45^{\circ}$ from neutral axis; and (e) $-90^{\circ}$ from neutral axis. Stresses determined from elastic-plastic FEA. 

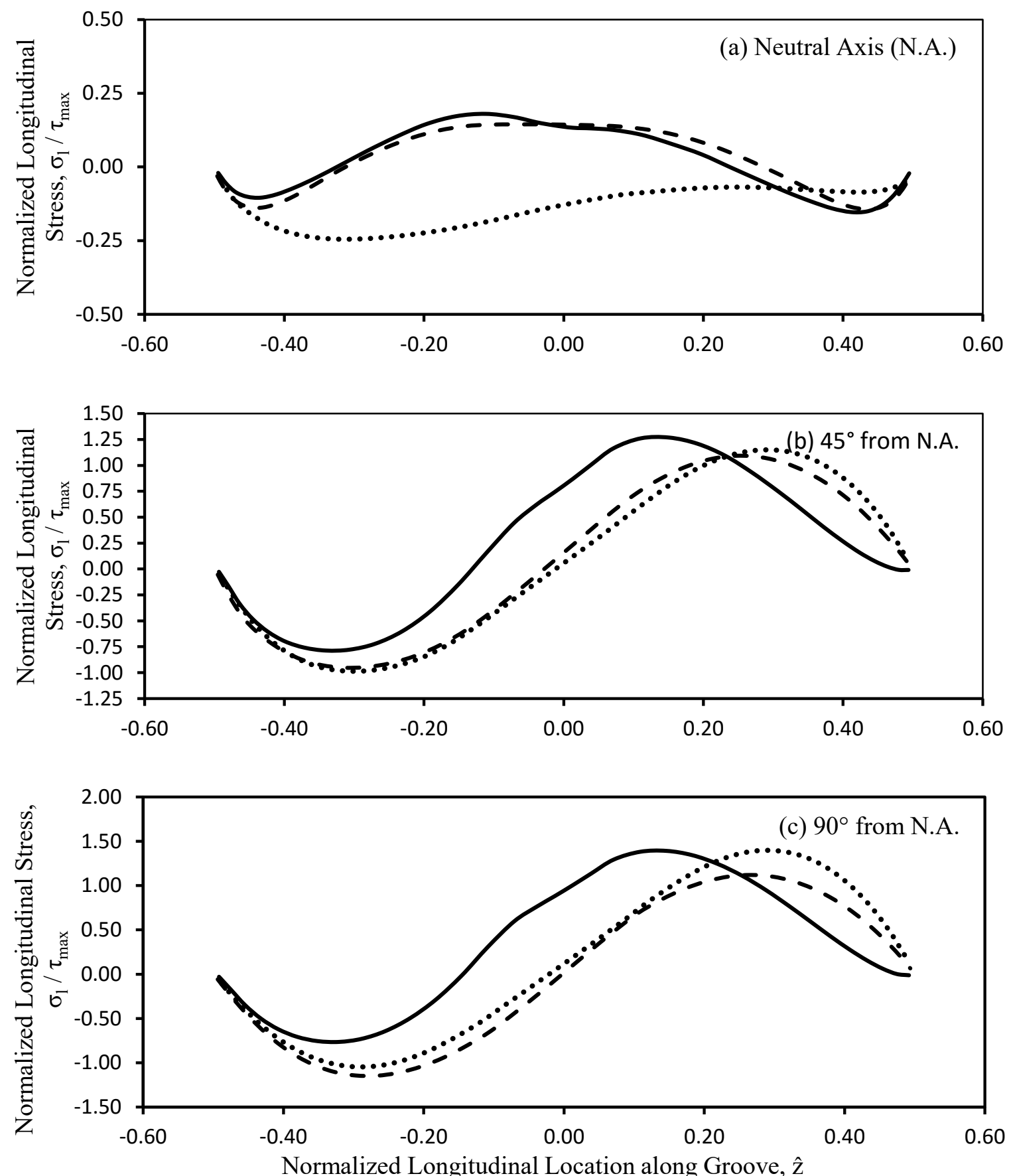

$\longrightarrow$ Pin A (Clevis+Bushing) - - Pin B (Cantilever+Bushing) ..... Pin C (Cantilever+Spherical)

Figure 6-2: Longitudinal stress distribution along fuse pin groove at (a) neutral axis; (b) $45^{\circ}$ from neutral axis; (c) $90^{\circ}$ from neutral axis; (d) $-45^{\circ}$ from neutral axis; and (e) $-90^{\circ}$ from neutral axis. Stresses determined from elastic-plastic FEA. 

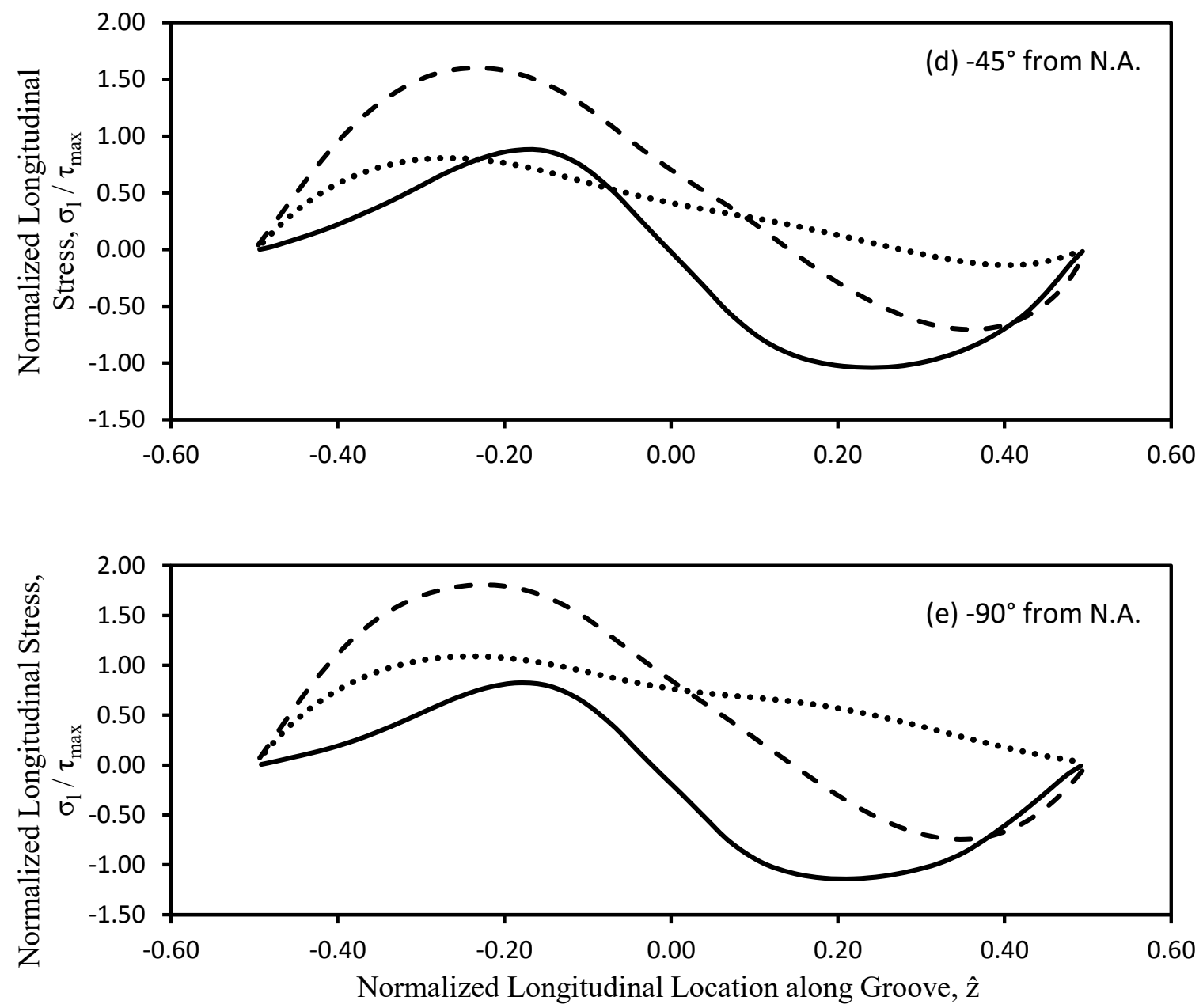

$\longrightarrow$ Pin A (Clevis+Bushing) - - Pin B (Cantilever+Bushing) ..... Pin C (Cantilever+Spherical)

Figure 6-2 (continued): Longitudinal stress distribution along fuse pin groove at (a) neutral axis; (b) $45^{\circ}$ from neutral axis; (c) $90^{\circ}$ from neutral axis; (d) $-45^{\circ}$ from neutral axis; and (e) $-90^{\circ}$ from neutral axis. Stresses determined from elastic-plastic FEA. 

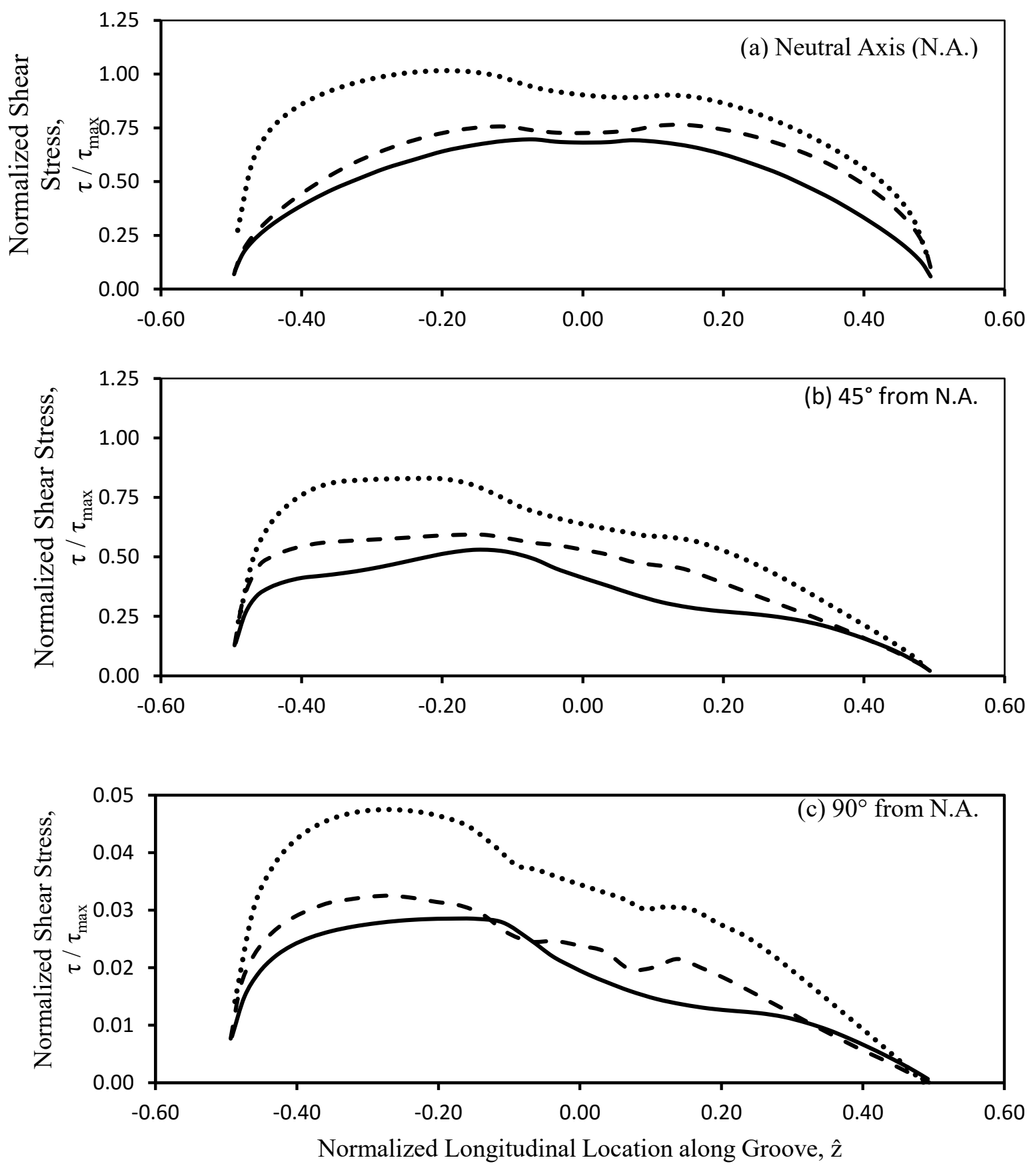

Pin A (Clevis+Bushing) - - • Pin B (Cantilever+Bushing) ...... Pin C (Cantilever+Spherical)

Figure 6-3: Shear stress distribution along fuse pin groove at (a) neutral axis; (b) $45^{\circ}$ from neutral axis; (c) $90^{\circ}$ from neutral axis; (d) $-45^{\circ}$ from neutral axis; and (e) $-90^{\circ}$ from neutral axis. Stresses determined from elastic-plastic FEA. 

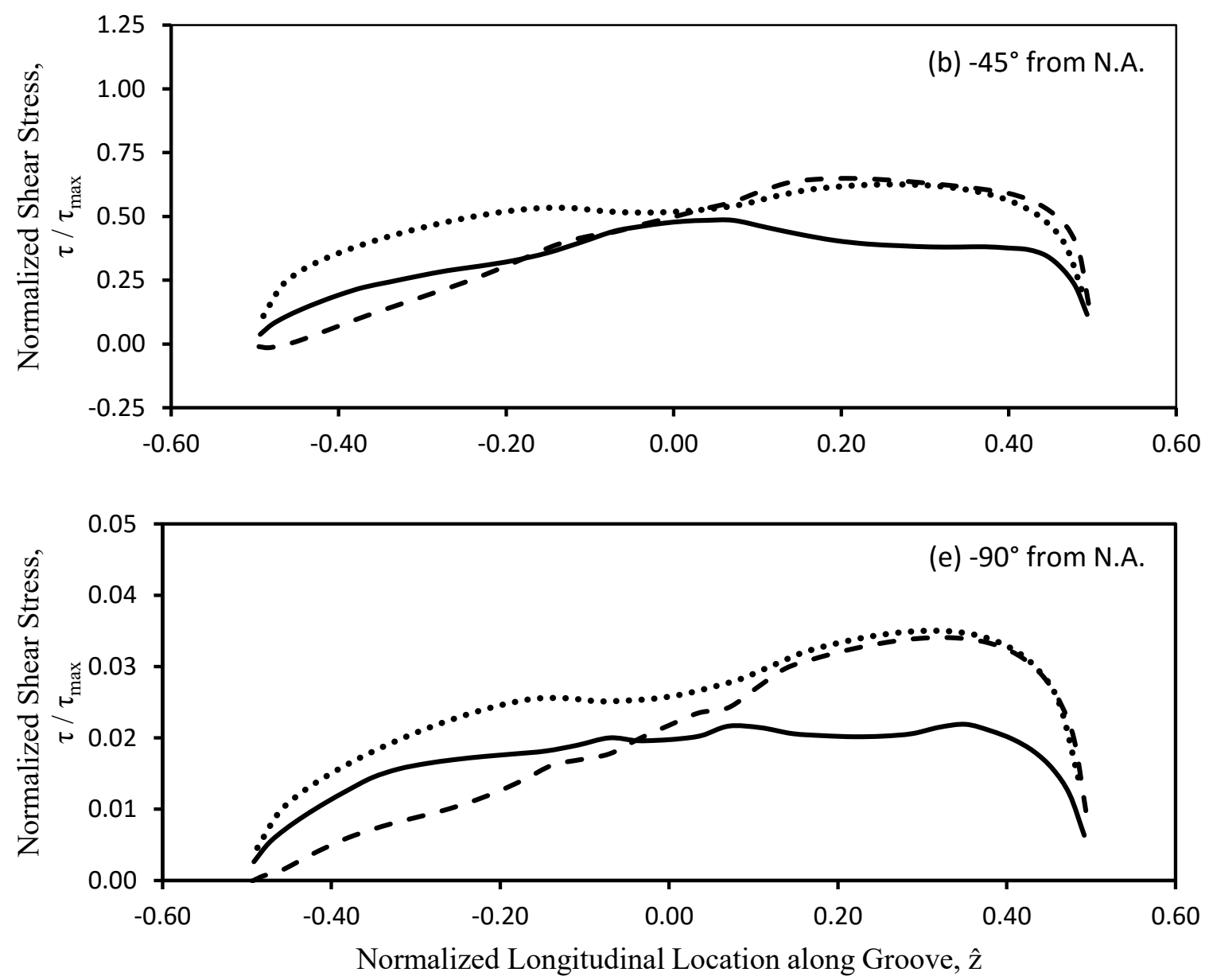

— Pin A (Clevis+Bushing) - — Pin B (Cantilever+Bushing) ..... Pin C (Cantilever+Spherical)

Figure 6-3 (continued): Shear stress distribution along fuse pin groove at (a) neutral axis; (b) $45^{\circ}$ from neutral axis; (c) $90^{\circ}$ from neutral axis; (d) $-45^{\circ}$ from neutral axis; and (e) $-90^{\circ}$ from neutral axis. Stresses determined from elastic-plastic FEA. 


\subsection{Methodology}

A fatigue analysis was performed for each of the three test fuse pins using different combinations of notch correction methods, cycle counting methods, and fatigue damage criteria introduced in the previous chapters. The different options for each of the elements of the fatigue analysis are summarized in Table 6-2.

Table 6-2: Summary of categories of the different elements of fatigue analysis.

\begin{tabular}{|l|l|l|l|}
\hline & $\begin{array}{l}\text { Notch } \\
\text { Correction }\end{array}$ & & \\
Fuse Pins & Methods & Cycle Counting Methods & Fatigue Damage Criteria \\
\hline Pin A & ESED & Wang-Brown & Kandil-Brown-Miller (KBM) \\
Pin B & Neuber & Wang-Brown Modified & Fatemi-Socie (FS) \\
Pin C & Unified & $\begin{array}{l}\text { Bannantine-Socie (E23) } \\
\text { Smith-Watson-Topper (SWT) }\end{array}$ & $\begin{array}{l}\text { Smantine-Socie (E22) } \\
\text { Biu Virtual Strain Energy (VSE) }\end{array}$ \\
\hline
\end{tabular}

The load history for each fuse pin was provided by UTAS-LS. Pin A was analyzed for 8444 reversals, Pin B was analyzed for 1122 reversals, and Pin C was analyzed for 2968 reversals. From the elastic FEA results provided by UTAS-LS, unit stresses (stress per unit lug load) for the hoop, longitudinal, and shear stress were determined for each of the fuse pins for each location along the circumference of the fuse pin; unit stresses and results determined through this procedure are referred to as FEA-based. The unit stresses are alternatively determined through the strain gauge readings determined from the actual tests for each fuse pin; results found using these unit stresses are referred to as test-based.

A total of five angular locations were analyzed along the circumference of the fuse groove: at the neutral axis $\left(0^{\circ}\right)$, at $\pm 45^{\circ}$ to the neutral axis, and at $\pm 90^{\circ}$ to the neutral axis. At each of the angular locations, excluding the neutral axis, a fatigue analysis was performed for a point before, after, and along the centerline of the fuse groove. The locations of maximum longitudinal stress in each of the pins are analyzed at each of the angular positions. For all of the 13 locations mentioned, FEA- 
based unit stresses ${ }^{2}$ were determined while test-based unit stresses were only determined for the neutral axis. Test-based unit stresses ${ }^{3}$ were not determined for other locations due to the inability of the strain gauges to detect the sharp changes in strain along the width of the fuse groove.

\subsection{Results of Multiaxial Fatigue Analysis}

A complete fatigue analysis was performed for each of the fuse pins at 13 different locations in the fuse groove. This section presents the strain results and fatigue damage results as predicted using the multiaxial fatigue program which incorporates the three different elements of the fatigue analysis process.

\subsubsection{Stress-Strain Predictions}

The first stage of fatigue analysis procedure is calculating the elastic-plastic stresses and strains using the stress-strain model which includes the Jiang-Sehitoglu kinematic hardening model defined in Section 2.3.2 and the notch correction methods from Section 2.3.3. During the analysis, it was found that the amount of non-proportional hardening was insignificant for the given load history and thus the non-proportional hardening factor $\Phi$ was artificially set to a value of zero for all results presented in this section.

Significant plasticity was found for two locations: at the neutral axis in Pin A and at the neutral axis in Pin B. The load at which yielding is expected to occur in the pin, referred to as the limit load, was determined from the elastic FEA for each pin. The maximum load in the load history used for Pin A is approximately $148 \%$ of the limit load, while the maximum load for Pin B is approximately $104 \%$ of the limit load and the Pin C loads caused no plasticity.

The strains predicted by the stress-strain model using FEA-based unit stresses are plotted against the experimental strain in Figure 6-4 through Figure 6-6, Figure 6-7 through Figure 6-9, and Figure 6-10 through Figure 6-12 for predictions made using the ESED, Neuber, and Unified notch correction methods, respectively, for Pin A. Figure 6-13 through Figure 6-15 show the predicted

\footnotetext{
${ }^{2}$ Due to the proprietary nature of the work, the FEA-based unit stresses cannot be provided in this thesis.

${ }^{3}$ Due to the proprietary nature of the work, the test-based unit stresses cannot be provided in this thesis.
} 
strains compared to the experimental strains for Pin B. All strain values are total strain measurements and predictions. In each of these figures, the strain for every thousand load cases is plotted to show the progression of the strain predictions and experimental values. No plots are provided for Pin $\mathrm{C}$ and other locations inside the fuse groove in Pin A and B due to there being little or no plastic deformation.

For all three correction methods, significant ratchetting is observed in Pin A in the experimental longitudinal strain that cannot be replicated by the current implementation of the Jiang-Sehitoglu stress-strain model. In Figure 6-4 for the ESED-based predictions, it is clear that the predicted strain tends towards a stabilized result while the experimental longitudinal strains continue to increase. It is noted here that the Jiang-Sehitoglu model was implemented so as to neglect ratchetting effects, as discussed in Chapter 3, and thus this type of correlation was somewhat expected for this particular case. In Figure 6-7 for the Neuber-based predictions it is observed that the predicted strains actually show a reversed accumulation of longitudinal strain where the predicted strain becomes increasingly compressive while the experimental longitudinal strain becomes increasingly tensile. A similar behaviour is observed for the Unified notch correctionbased predictions in Figure 6-10. This demonstrates a deficiency in the implementation of the stress-strain model where significant ratchetting effects are not being properly modeled.

Figure 6-5 shows reasonable prediction of the hoop strain using the ESED notch correction method for Pin A, where all predicted values are within roughly $20 \%$ of the experimental results. The analyses using the Neuber and Unified notch correction method shows less stable results in comparison. In Figure 6-8 and Figure 6-11, the hoop strain predicted by the stress-strain model appears to drift in the tensile direction while no such tendency is observed experimentally.

For all notch correction methods, the shear strain results show very good correlation with experimental results. As seen in Figure 6-6, Figure 6-9, and Figure 6-12, most shear strain predictions are within $10-20 \%$ of the experimental values. The success of these predictions can potentially outweigh the deficiencies found in the predictions of the longitudinal and hoop strains since the fuse pin stress history at the neutral axis is dominated by shear loading. In general, the 
shear strain range is more than double that of the longitudinal strain range in both the experimental and predicted results.

Having observed the behaviour of the implemented stress-strain model for Pin A, where the maximum load reaches $148 \%$ of the limit load, the behaviour of the stress-strain model can be further evaluated by considering Pin B, where the amount of the plastic deformation is significantly lower. Figure 6-13, Figure 6-14, Figure 6-15 show the strain predictions compared to the experimental results from the Pin B tests. All strain components are plotted on the same graph. No significant ratchetting effects are observed in the experimental data for Pin B and thus relatively stable strains are observed. The longitudinal and hoop strains are both under-predicted by up to $50 \%$. Significant over-prediction of the shear strain is observed for all three notch correction methods, reaching a maximum of roughly double the value of the experimental strains. Overall, the under- and over-prediction of the experimental strains remain consistent with respect to the load-level for all cases.

While the differences between the predicted data and experimental data are significant, much of these differences can be attributed to the FEA predictions of the unit stresses. Figure 6-16, Figure 6-17, and Figure 6-18 show the longitudinal, hoop, and shear stresses for Pin A as a function of the applied fuse pin load as calculated from the linear elastic FEA and from the experimental strain data. All stress values have been normalized by the maximum average shear stress of Pin A and the applied loads have been normalized by the maximum fuse pin load. The experimental longitudinal stresses for compressive loads deviate significantly from that which is predicted by the linear FEA. This may indicate either some systematic experimental error due to the application of the strain gauge at the bottom of the fuse grove or some assembly level non-linearity that the FEA does not reflect. The experimental hoop stresses follow the linear elastic FEA predictions much better; however there is still a slight deviation for both compressive and tensile loads. The shear stress, on the other hand, shows essentially exact correlation between linear elastic FEA and the experimental measurements. For Pin A, it is difficult to separate the errors due to the FEAbased unit stresses and the errors due to the stress-strain model because of the significant ratchetting that occurs. Pin B provides better insights into the difference between the FEA-based stresses and the test-based stresses. 


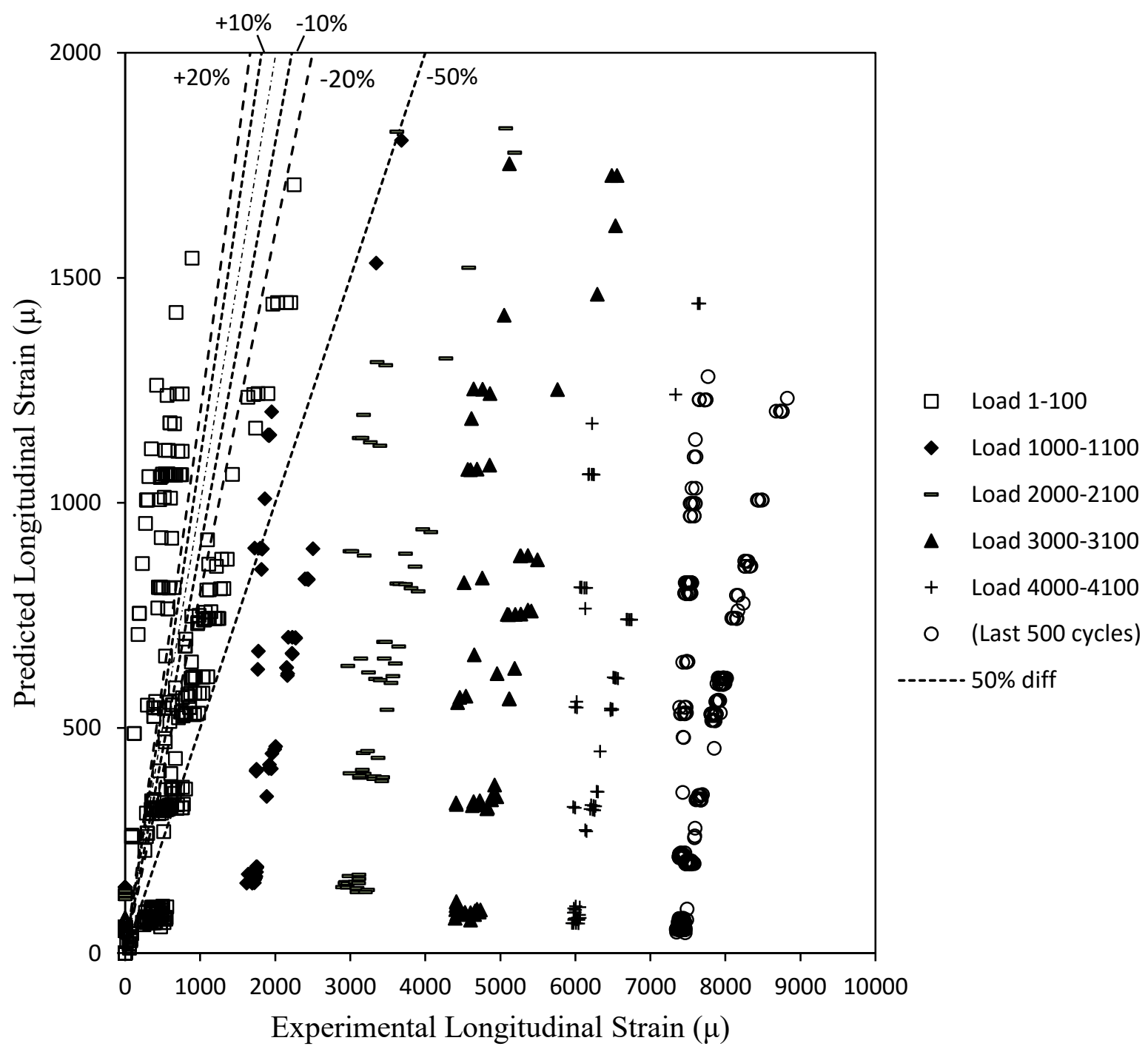

Figure 6-4: Longitudinal strain predicted by Jiang-Sehitoglu stress-strain model with suppressed non-proportional hardening and ESED notch correction compared to experimental longitudinal strain at the neutral axis and center of the fuse groove Pin A. 


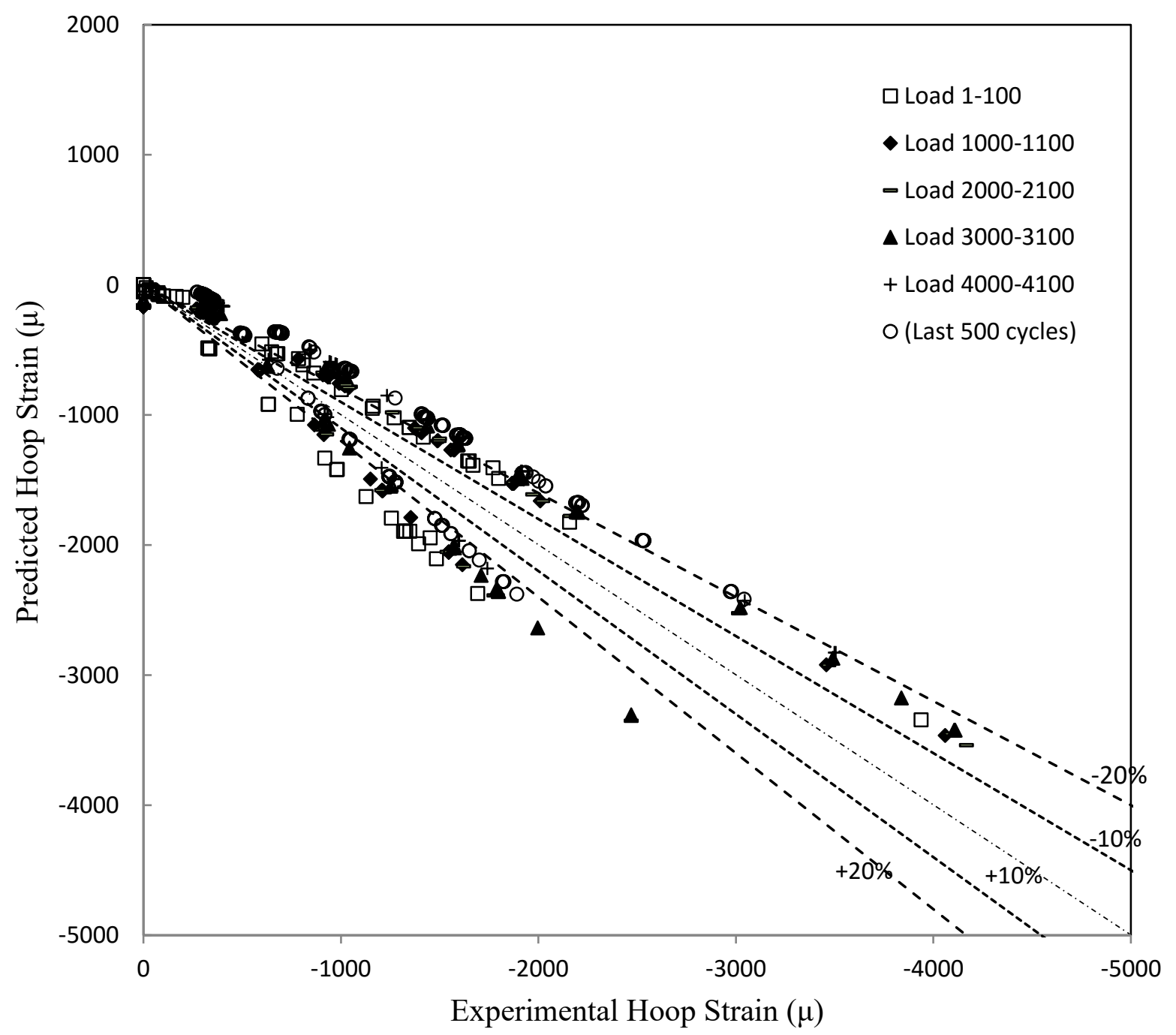

Figure 6-5: Hoop strain predicted by Jiang-Sehitoglu stress-strain model with suppressed nonproportional hardening and ESED notch correction compared to experimental hoop strain at the neutral axis and center of the fuse groove for Pin A. 


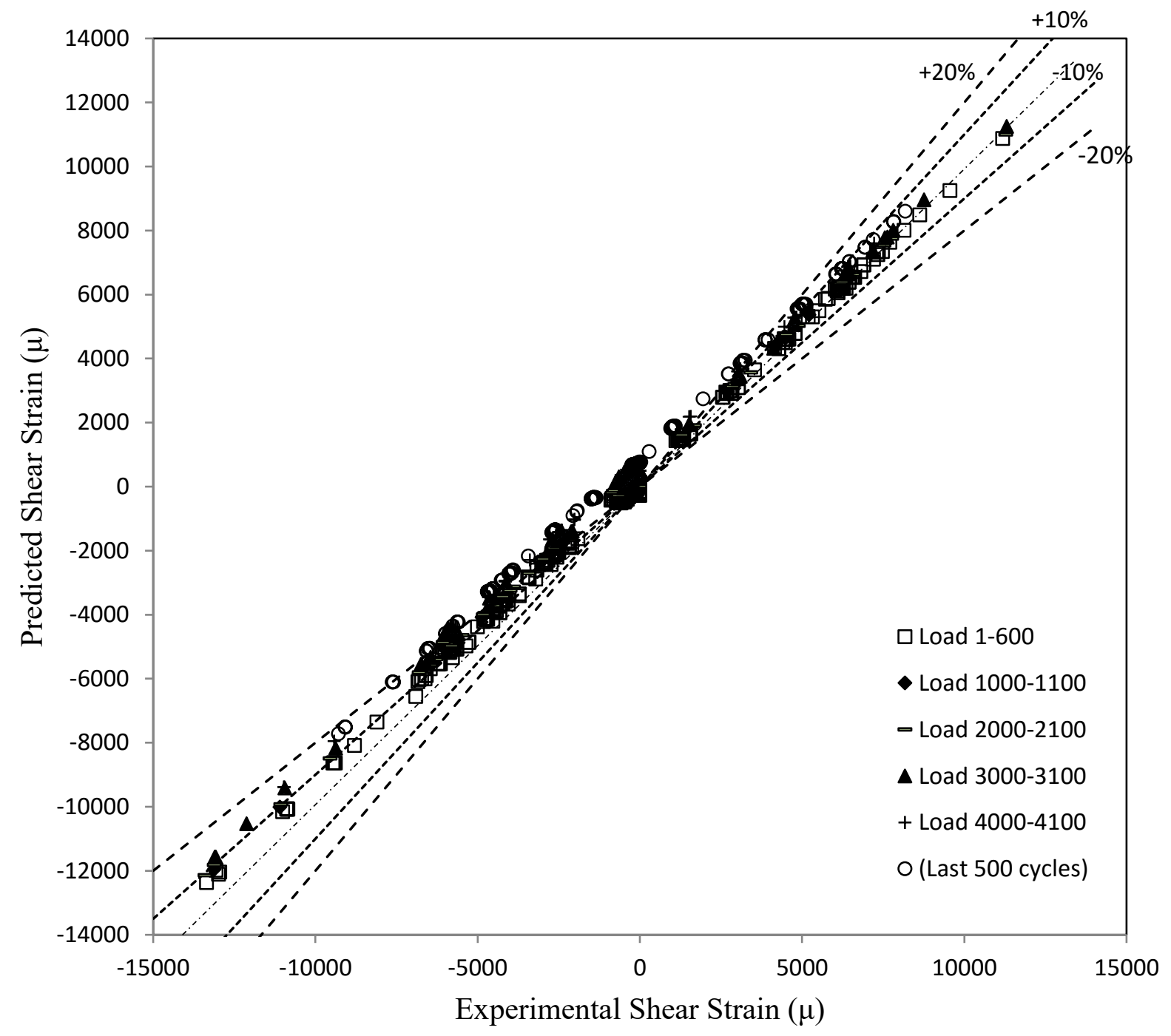

Figure 6-6: Shear strain predicted by Jiang-Sehitoglu stress-strain model with suppressed nonproportional hardening and ESED notch correction compared to experimental shear strain at the neutral axis and center of the fuse groove for Pin A. 


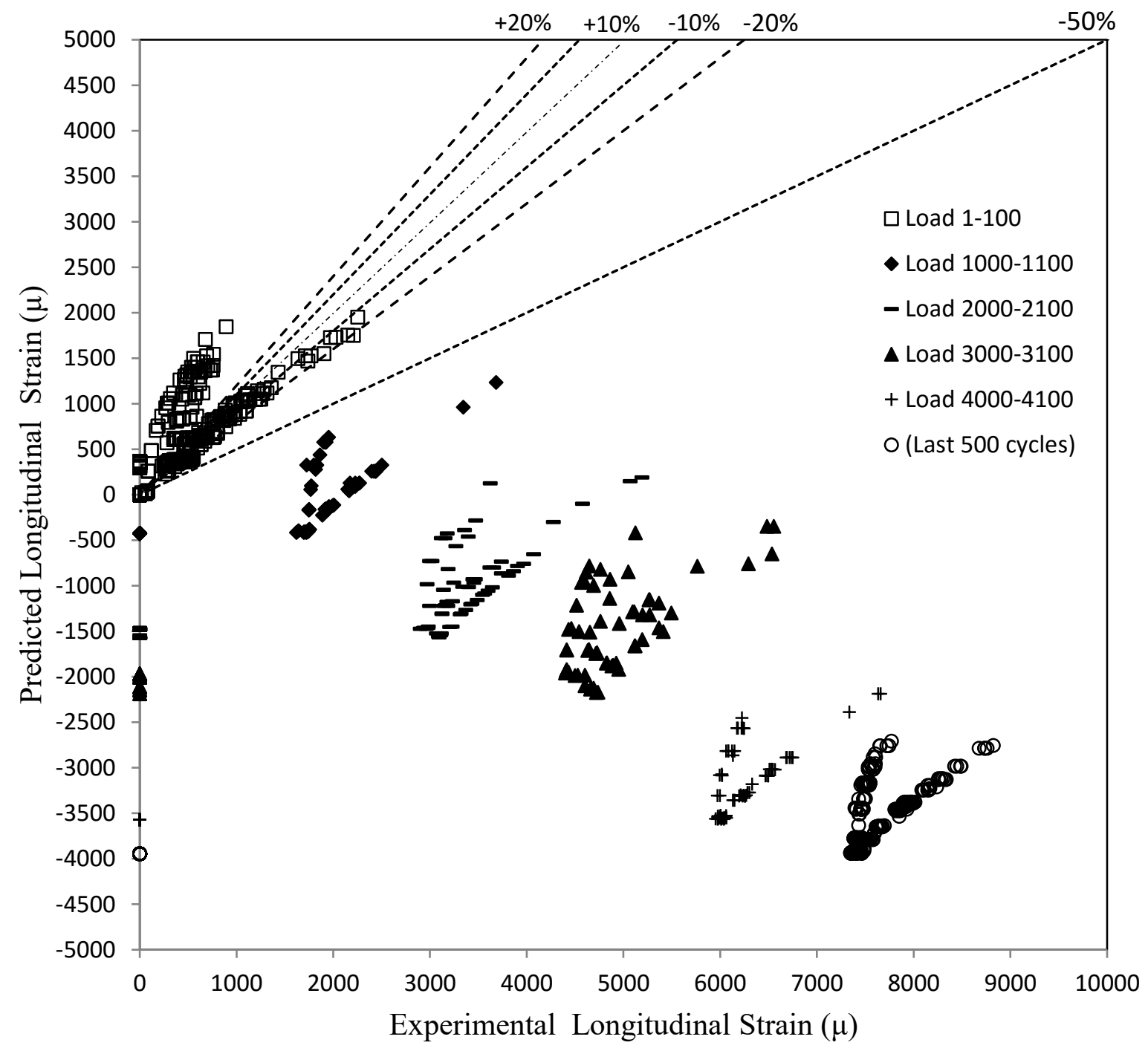

Figure 6-7: Longitudinal strain predicted by Jiang-Sehitoglu stress-strain model with suppressed non-proportional hardening and Neuber notch correction compared to experimental longitudinal strain at the neutral axis and center of the fuse groove for Pin A. 


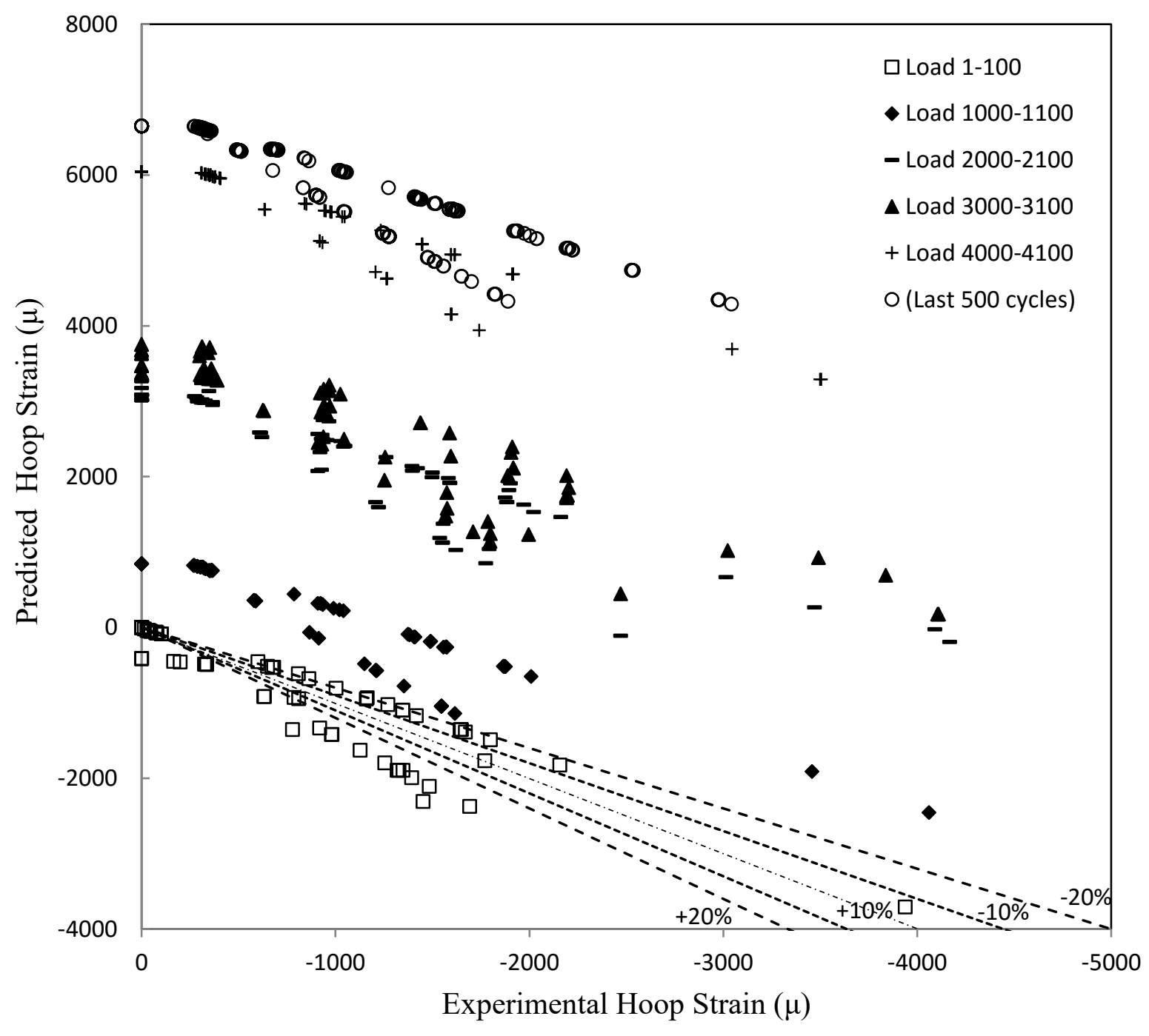

Figure 6-8: Hoop strain predicted by Jiang-Sehitoglu stress-strain model with suppressed nonproportional hardening and Neuber notch correction compared to experimental hoop strain at the neutral axis and center of the fuse groove for Pin A. 


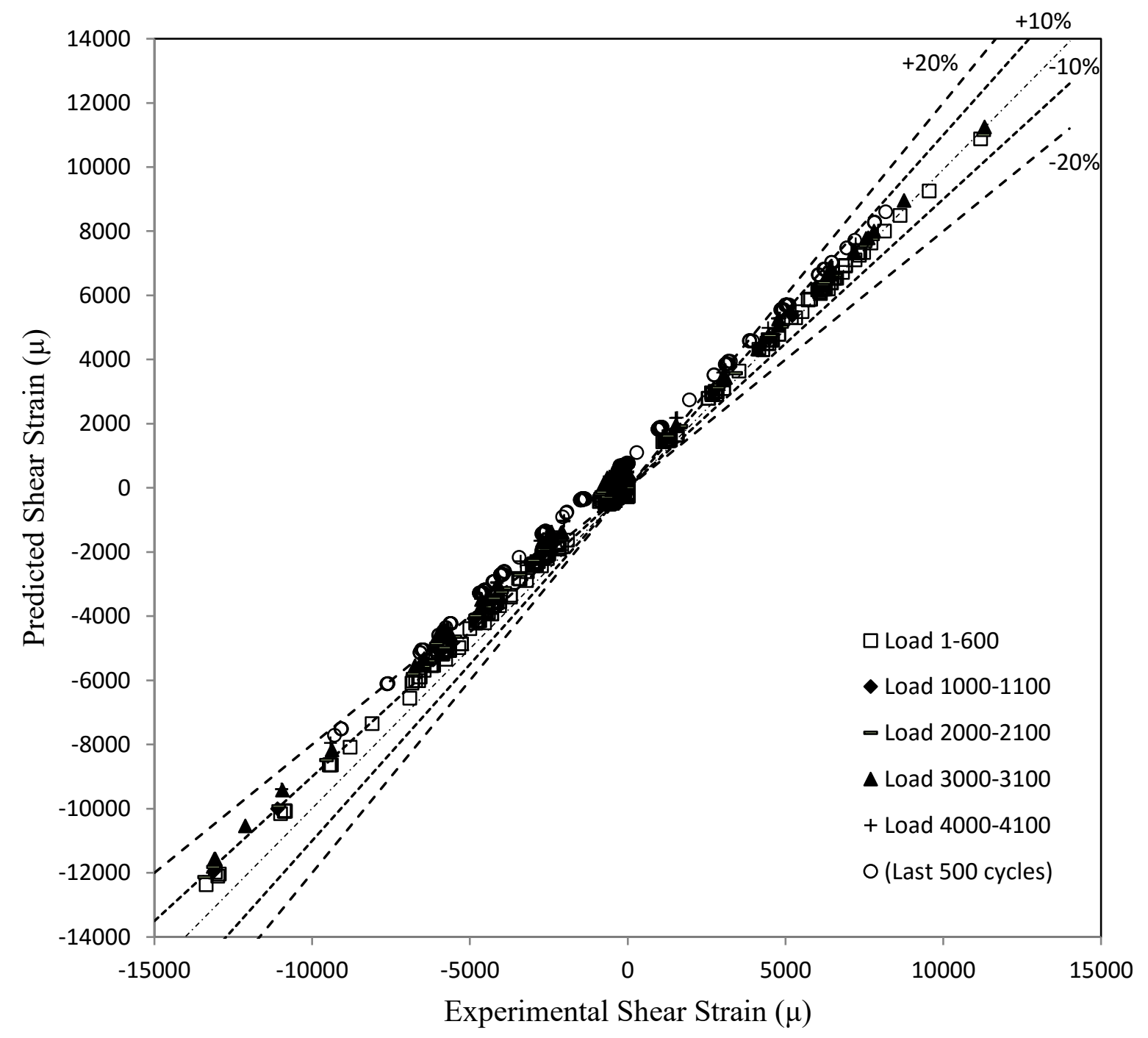

Figure 6-9: Shear strain predicted by Jiang-Sehitoglu stress-strain model with suppressed nonproportional hardening and Neuber notch correction compared to experimental shear strain at the neutral axis and center of the fuse groove for Pin A. 


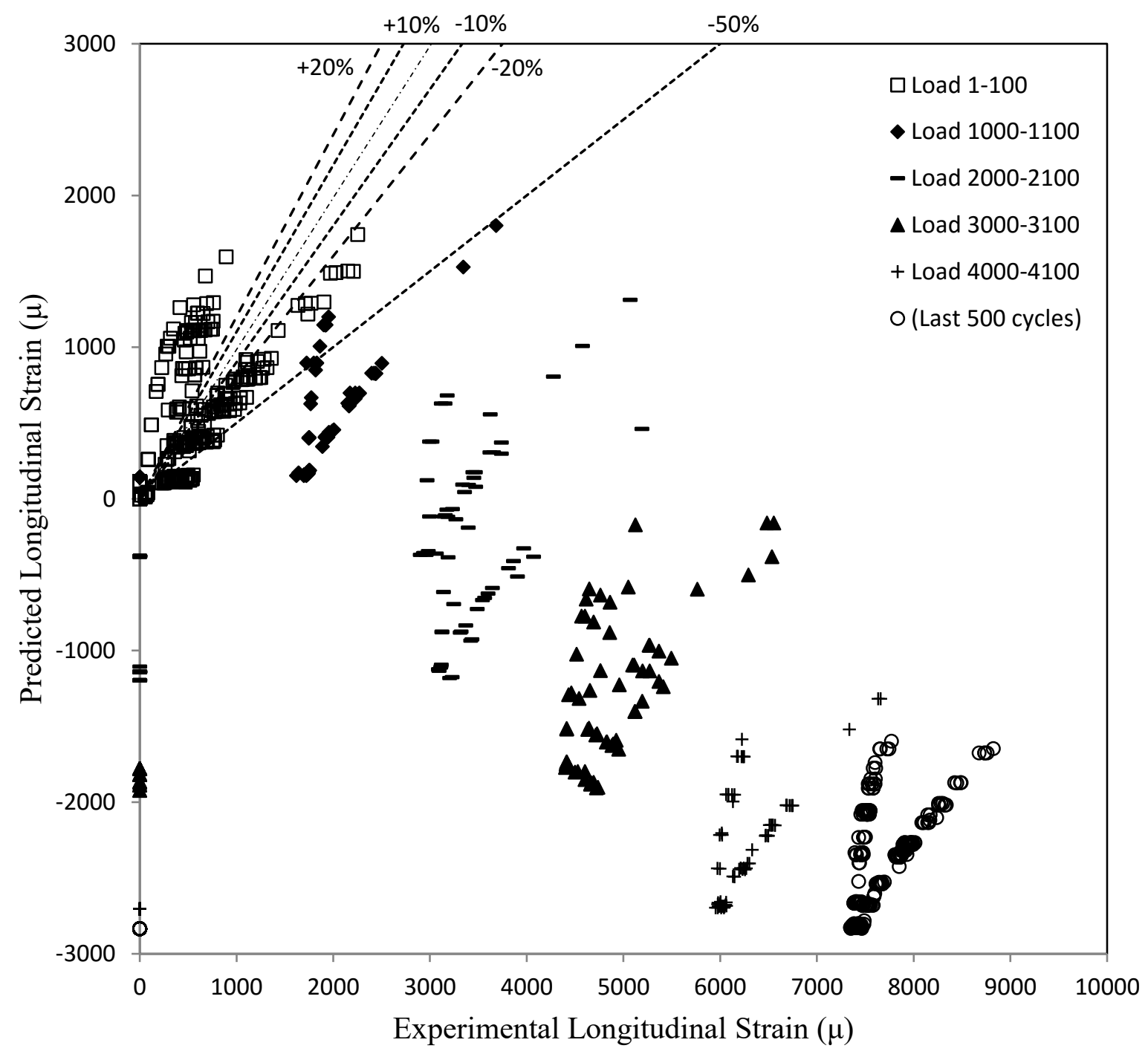

Figure 6-10: Longitudinal strain predicted by Jiang-Sehitoglu stress-strain model with suppressed non-proportional hardening and Unified notch correction compared to experimental longitudinal strain at the neutral axis and center of the fuse groove for Pin A. 


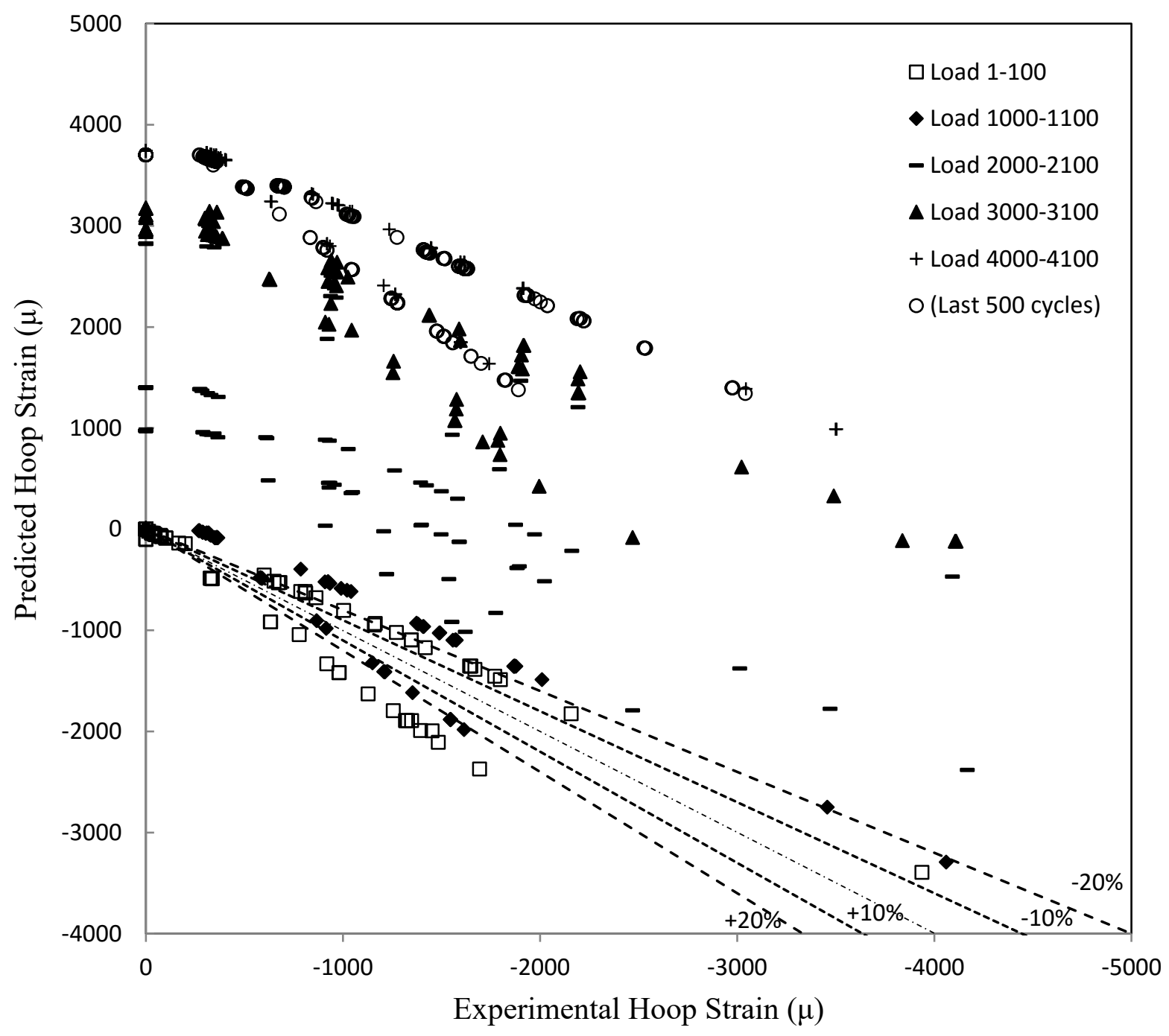

Figure 6-11: Hoop strain predicted by Jiang-Sehitoglu stress-strain model with suppressed nonproportional hardening and Unified notch correction compared to experimental hoop strain at the neutral axis and center of the fuse groove for Pin A. 


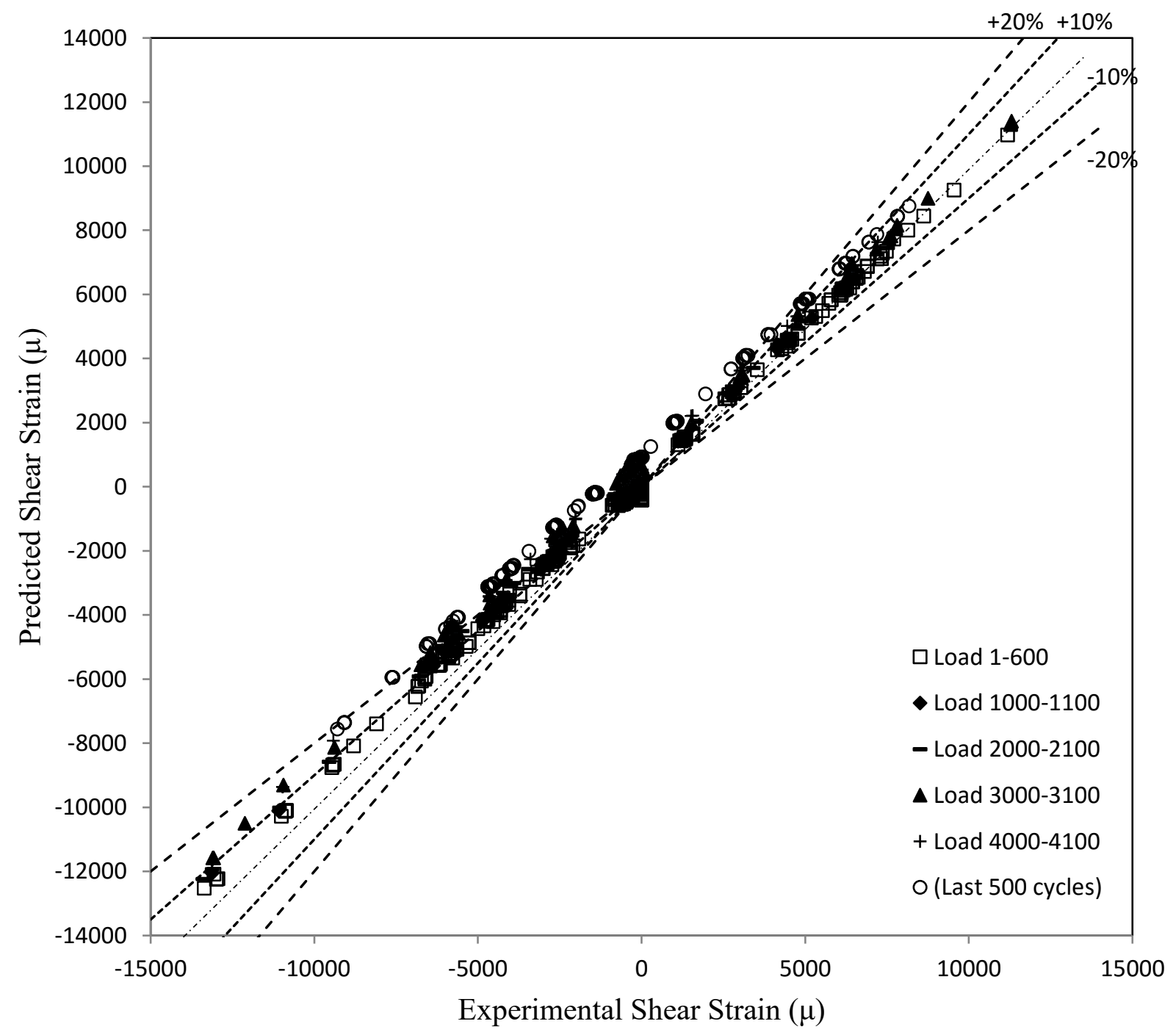

Figure 6-12: Shear strain predicted by Jiang-Sehitoglu stress-strain model with suppressed nonproportional hardening and Unified notch correction compared to experimental shear strain at the neutral axis and center of the fuse groove for Pin A. 


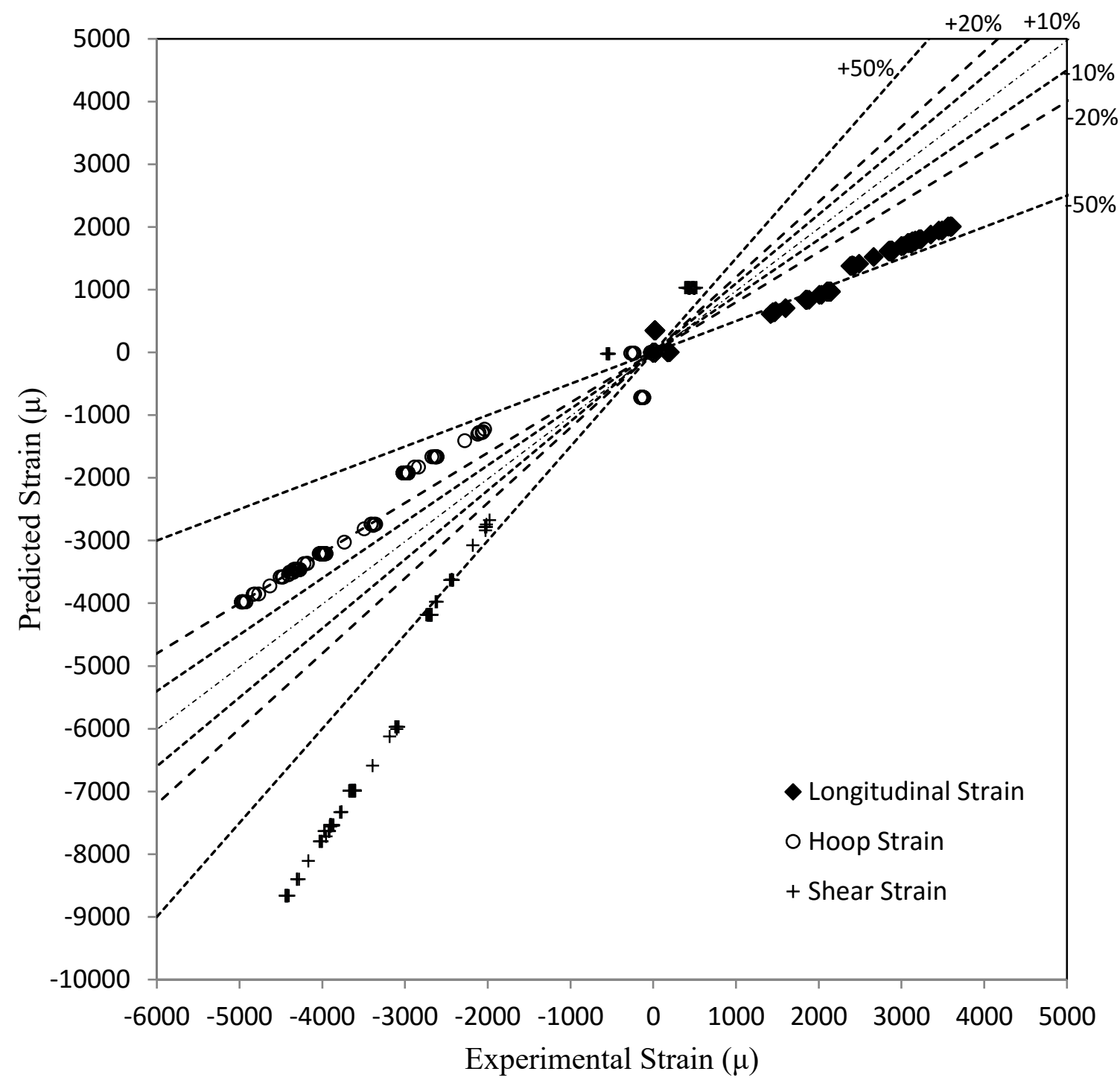

Figure 6-13: Longitudinal, hoop, and shear strain predicted by Jiang-Sehitoglu stress-strain model with suppressed non-proportional hardening and ESED notch correction compared to experimental strain at the neutral axis and center of the fuse groove for Pin B. Note that all data points from the strain history are included in this chart. 


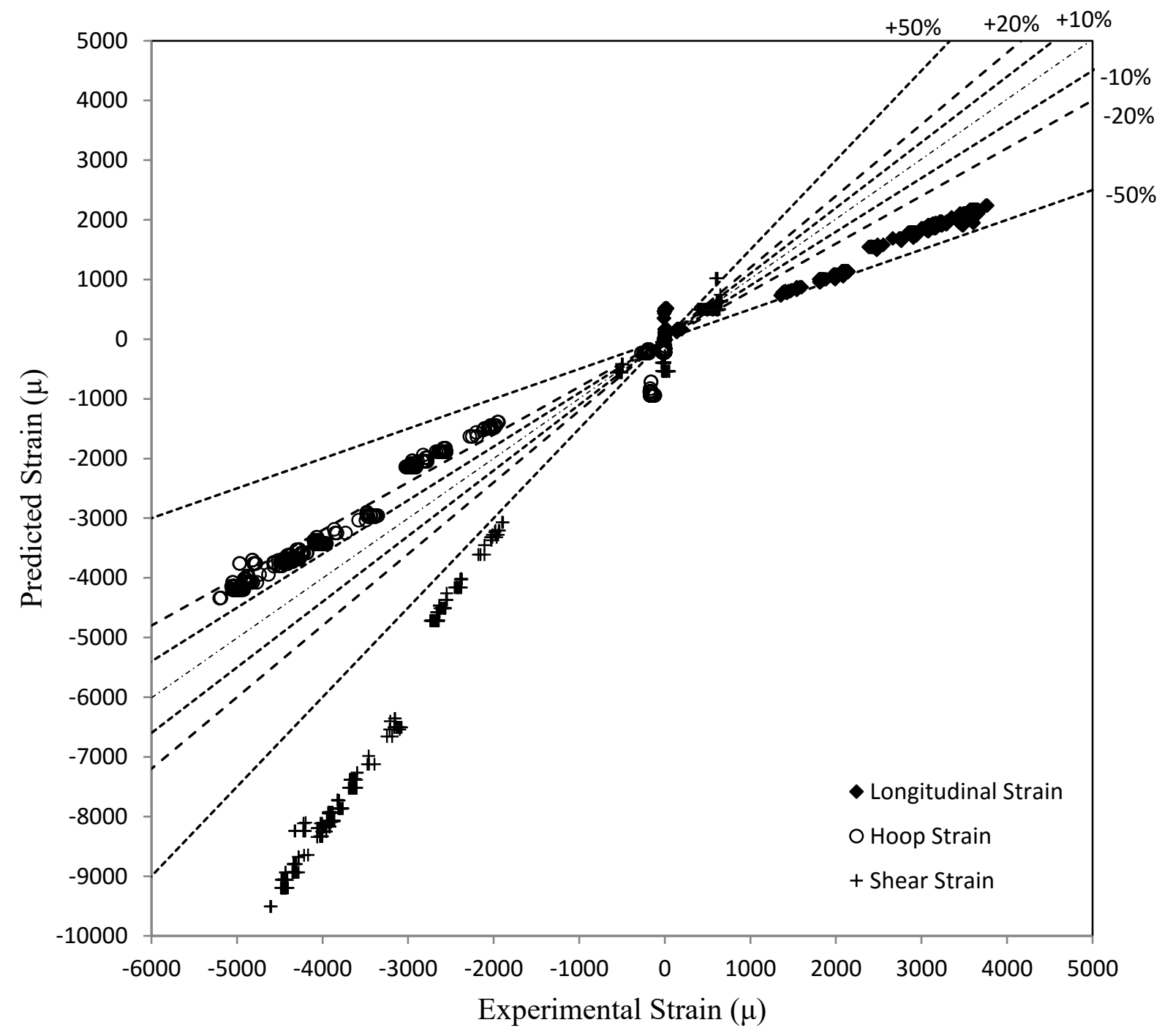

Figure 6-14: Longitudinal, hoop, and shear strain predicted by Jiang-Sehitoglu stress-strain model with suppressed non-proportional hardening and Neuber notch correction compared to experimental strain at the neutral axis and center of the fuse groove for Pin B. Note that all data points from the strain history are included in this chart. 


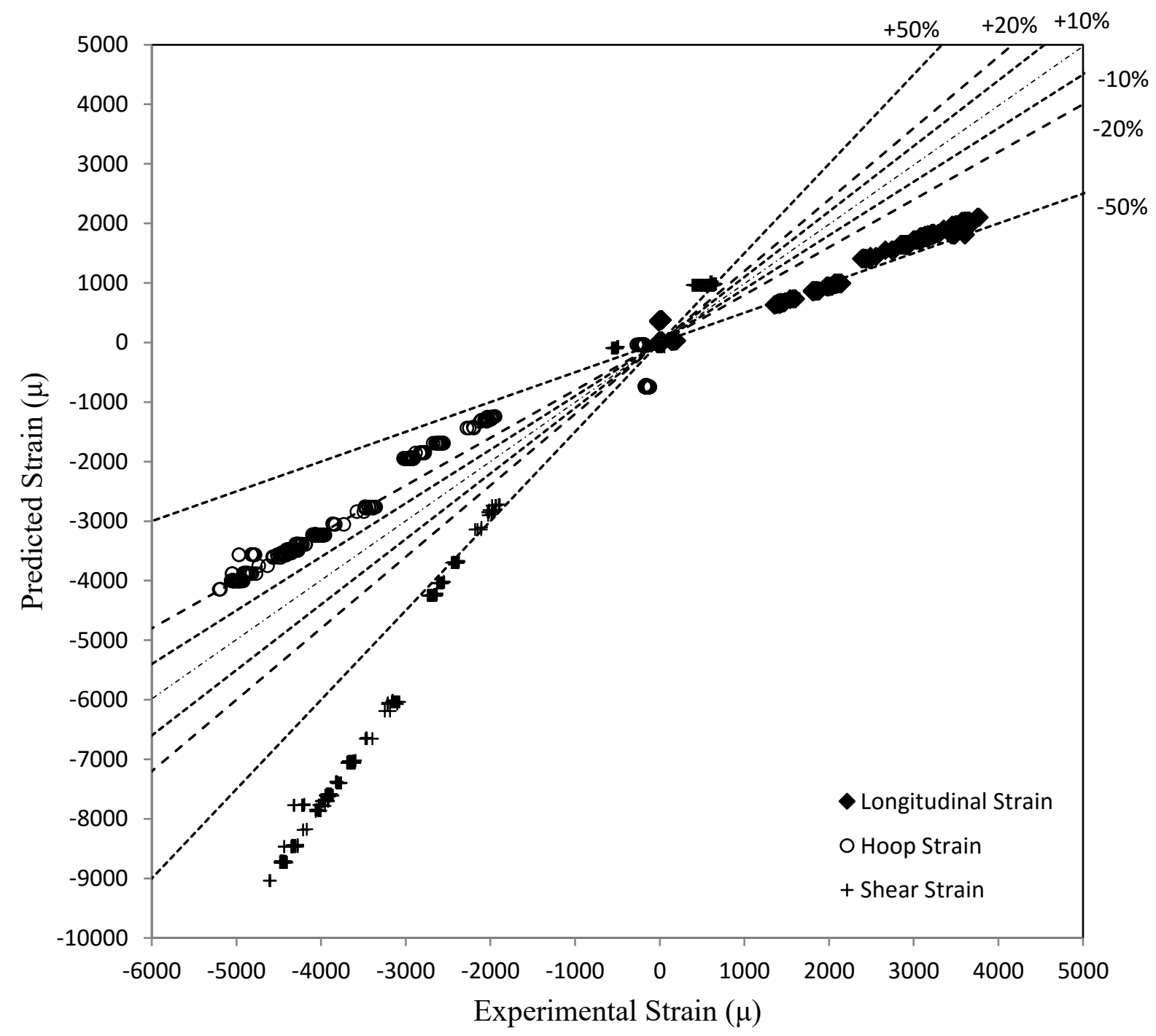

Figure 6-15: Longitudinal, hoop, and shear strain predicted by Jiang-Sehitoglu stress-strain model with suppressed non-proportional hardening and Unified notch correction compared to experimental strain at the neutral axis and center of the fuse groove for Pin B. Note that all data points from the strain history are included in this chart. 


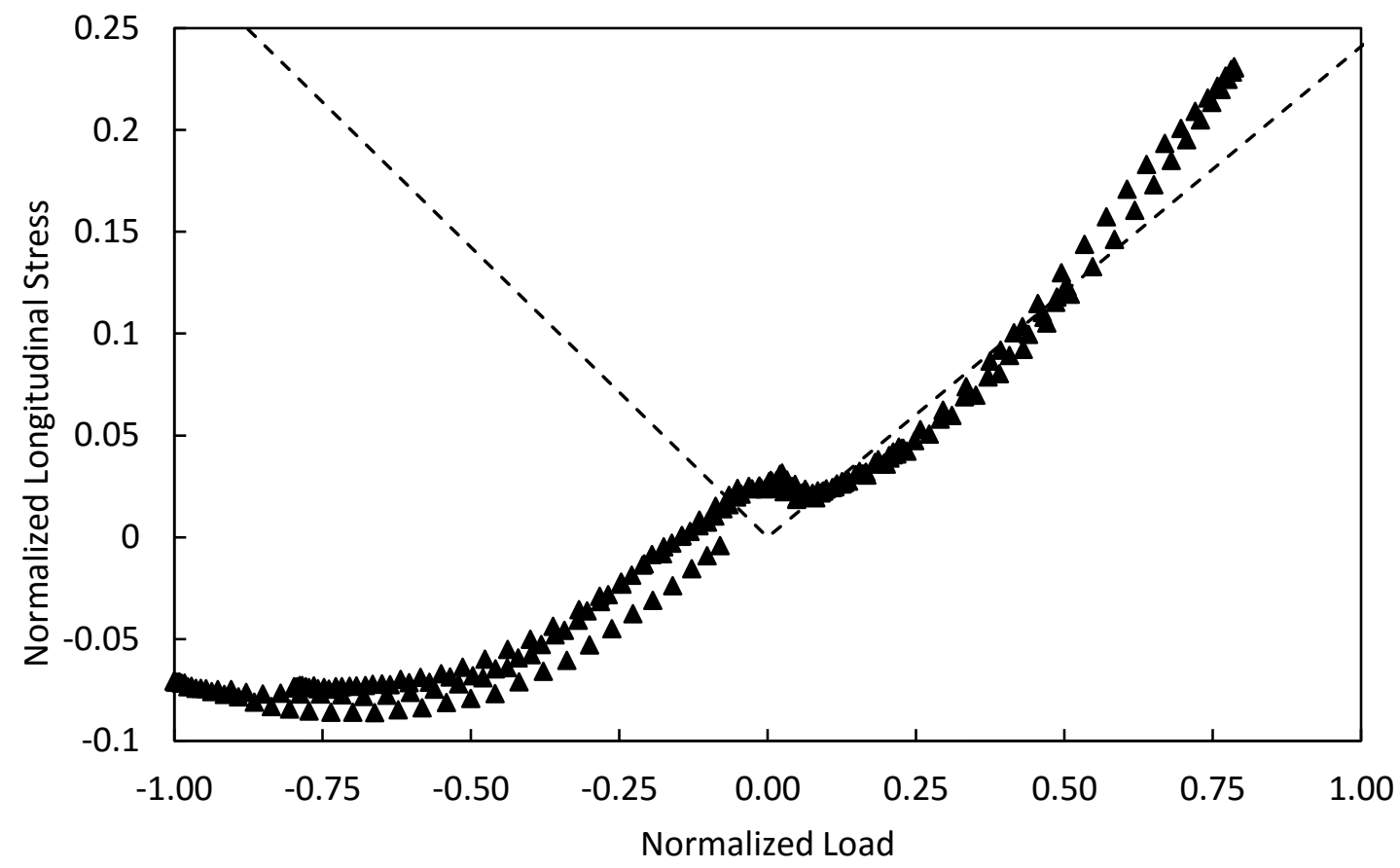

\ Experimental - - - Linear FE Interpolation

Figure 6-16: Longitudinal stress as predicted by linear elastic FEA and by experimental strain data for Pin A. Stress values have been normalized with respect to the maximum average shear stress in the fuse groove. 


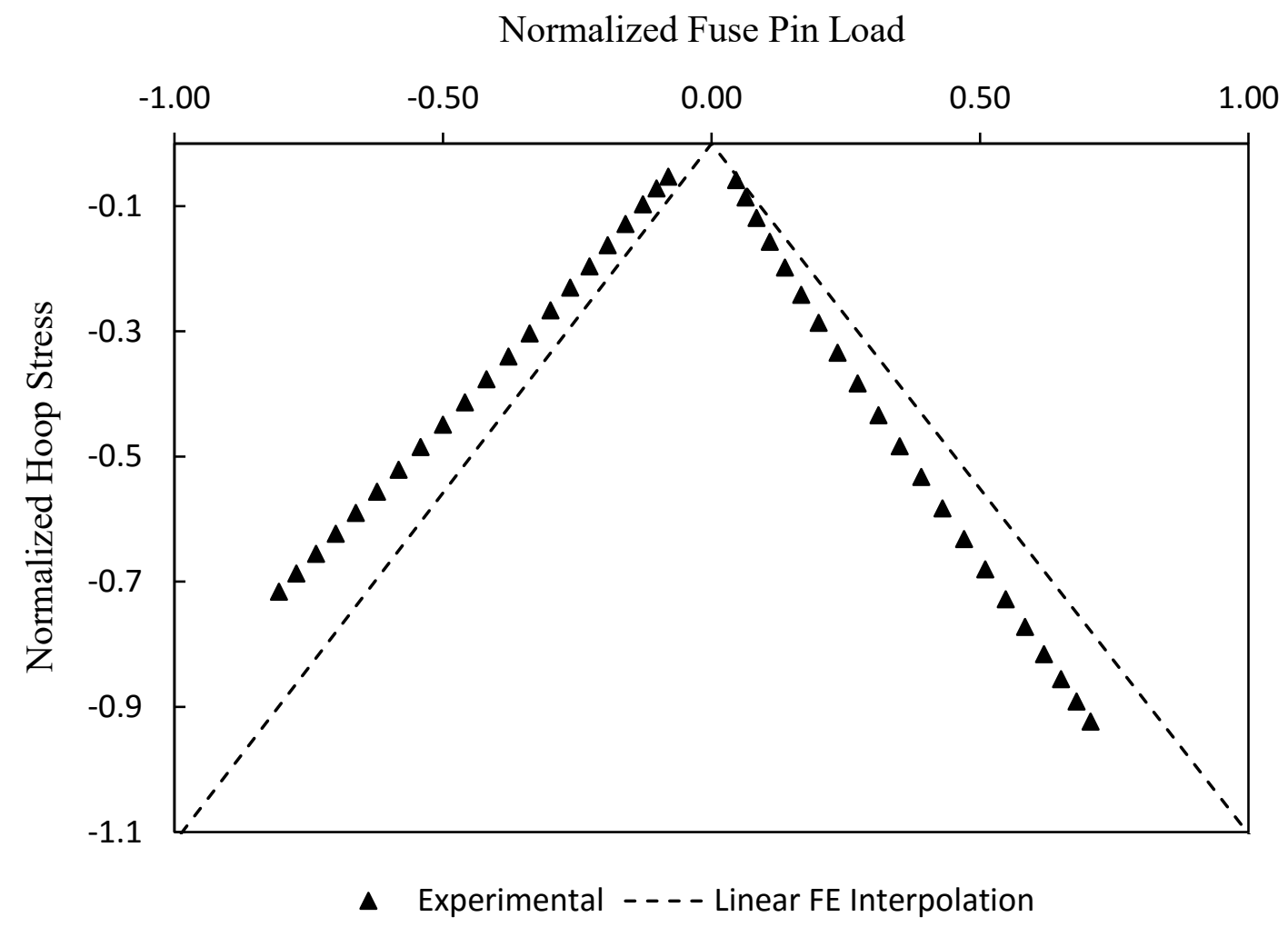

Figure 6-17: Hoop stress as predicted by linear elastic FEA and by experimental strain data for Pin A. Stress values have been normalized with respect to the maximum average shear stress in the fuse groove. 


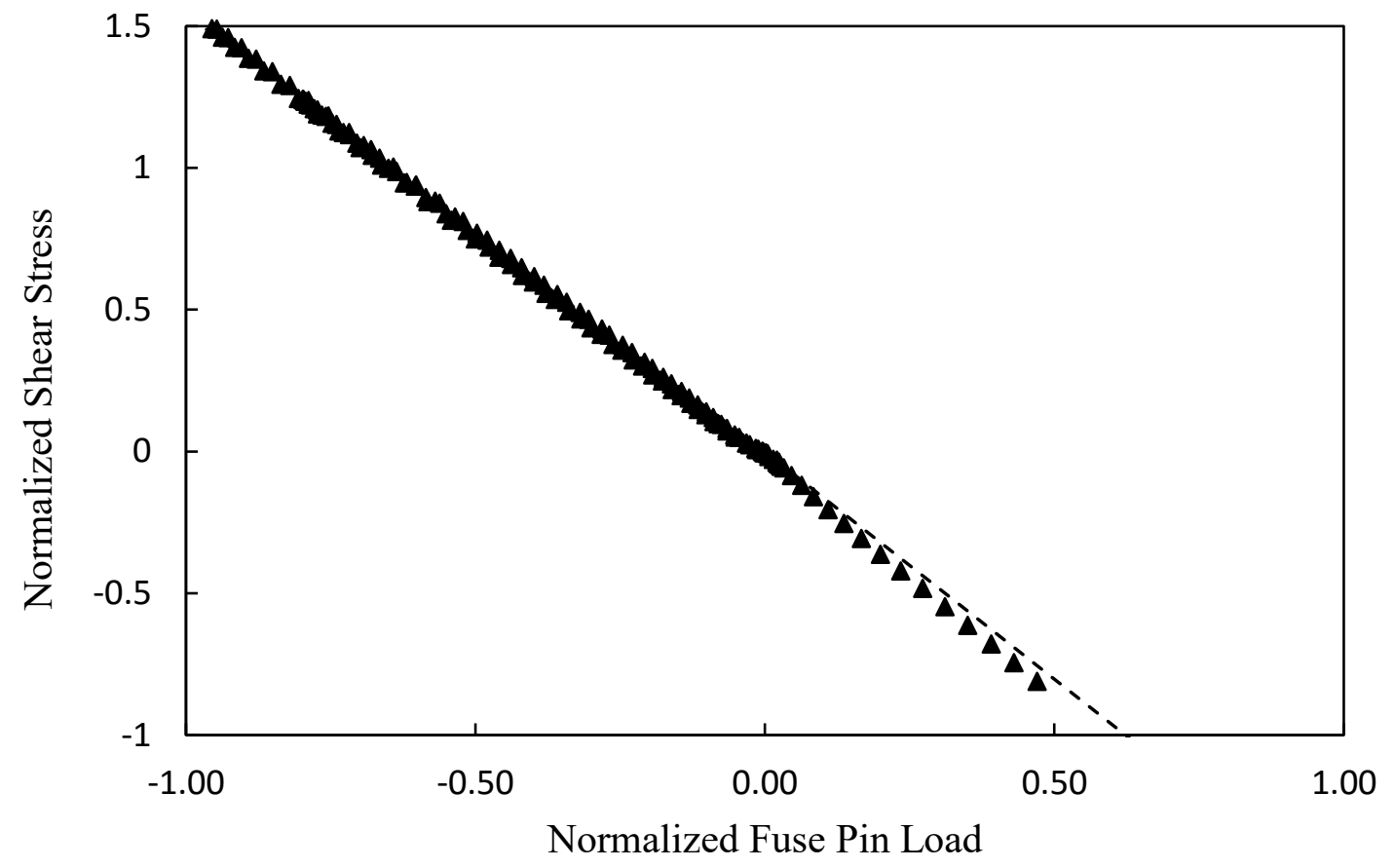

\ Experimental - - - Linear FE Interpolation

Figure 6-18: Shear stress as predicted by linear elastic FEA and by experimental strain data for Pin A. Stress values have been normalized with respect to the maximum average shear stress in the fuse groove. 


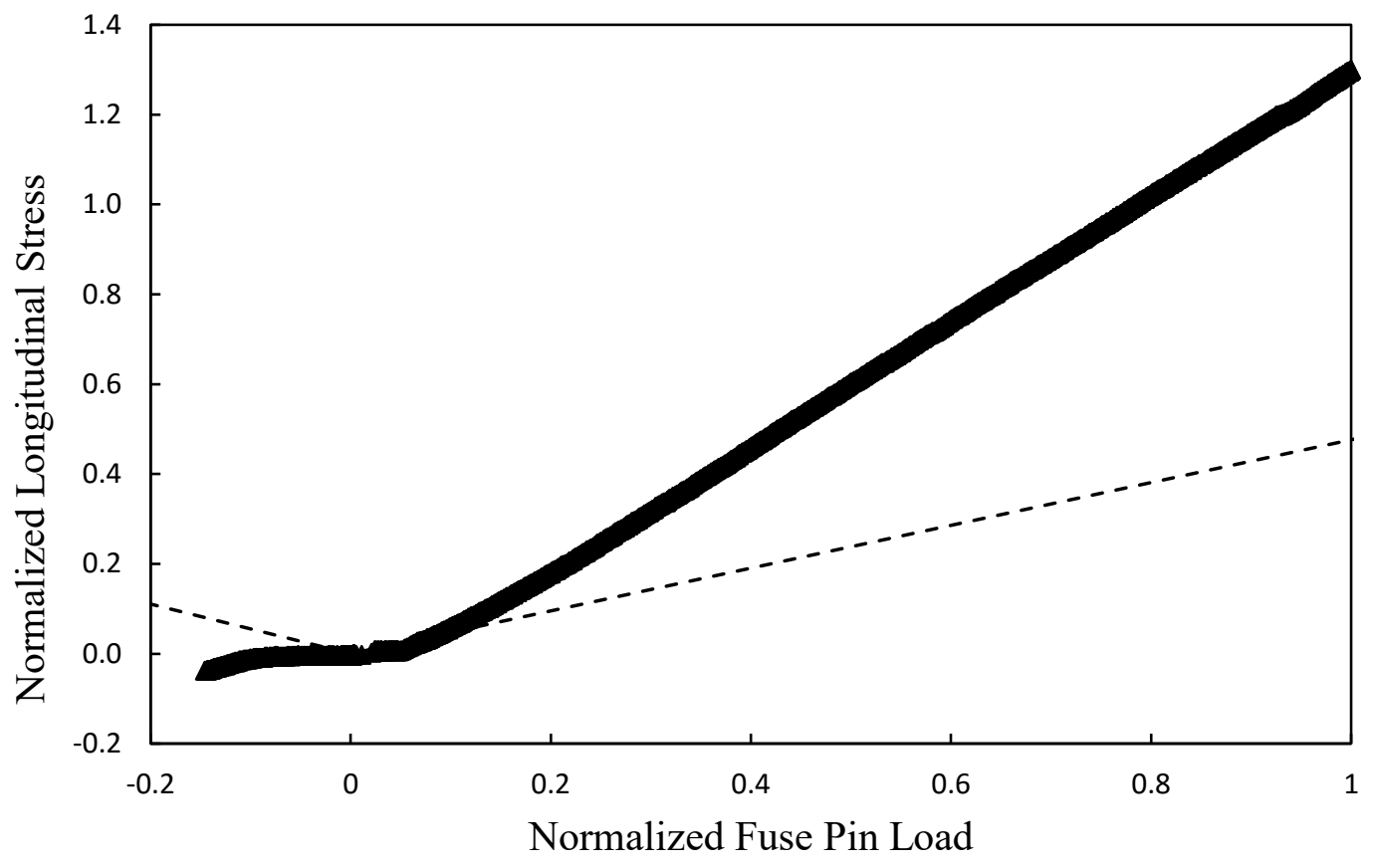

$\Delta$ Experimental - - - - Linear FE Interpolation

Figure 6-19: Longitudinal stress as predicted by linear elastic FEA and by experimental strain data for Pin B. Stress values have been normalized with respect to the maximum average shear stress in the fuse groove. 


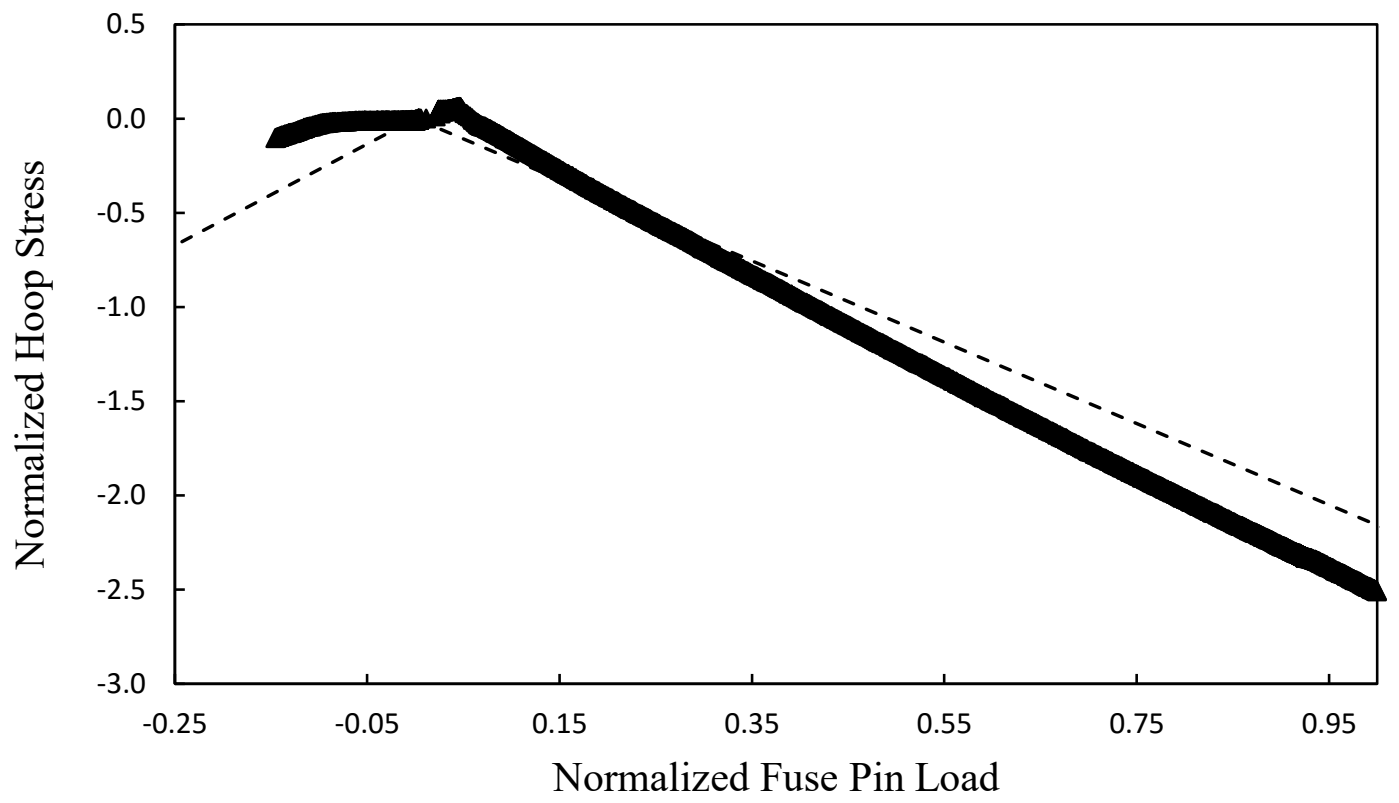

$\Delta$ Experimental - - - - Linear FE Interpolation

Figure 6-20: Hoop stress as predicted by linear elastic FEA and by experimental strain data for Pin B. Stress values have been normalized with respect to the maximum average shear stress in the fuse groove. 


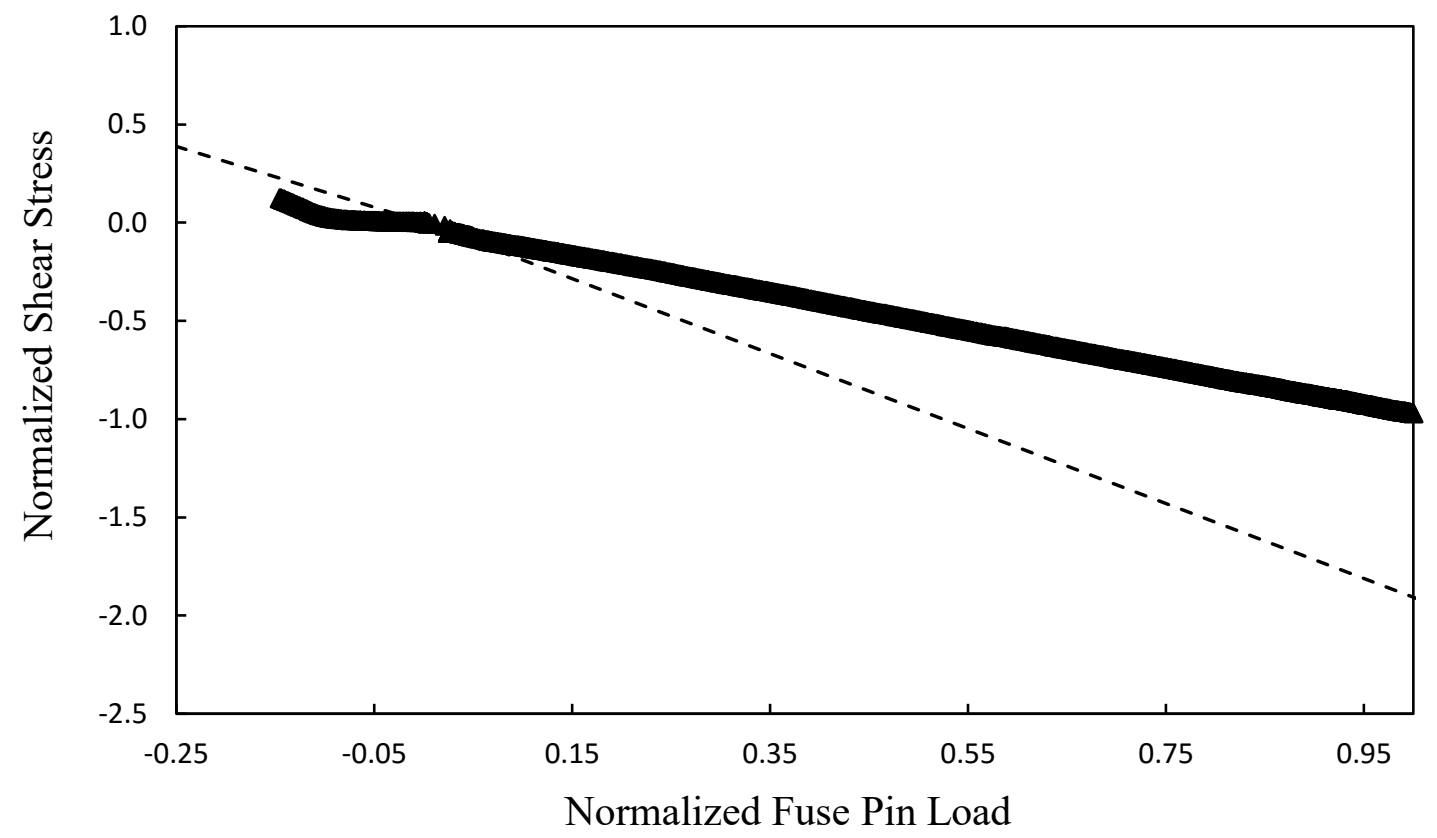

\ Shear Stress _ - - - Linear FE Interpolation

Figure 6-21: Shear stress as predicted by linear elastic FEA and by experimental strain data for Pin B. Stress values have been normalized with respect to the maximum average shear stress in the fuse groove. 


\subsubsection{Fatigue Damage Predictions}

\section{Results using UTAS-LS Uniaxial Fatigue Analysis Methodologies}

As a reference, fatigue damages predicted using UTAS-LS traditional methodologies are provided in Table 6-3 for the three pins. Three methodologies are compared: a pure shear method, an absolute max principal method, and a signed Von Mises method. All three methods use the uniaxial strain-life relation with the Smith-Watson-Topper mean stress correction presented as Eq.(2-18), the rainflow cycle counting procedure, and a modified Neuber's Rule which incorporates a residual stress calculation with each cycle. The pure shear method uses the simple average shear stress calculation at the centre of the fuse groove to determine the stresses for each cycle. While the pure shear method simply uses the fuse pin load and pin geometry to determine the stress, the absolute max principal and signed Von Mises methods use FEA results to calculate the stresses at every location in the fuse groove. The absolute max principal method uses the absolute maximum principal stress at a given location in the fuse groove to determine the peak stress at that location. The signed Von Mises method uses the von Mises as the peak stress at any given location in the fuse groove and adopts its sign from the direction of the shear stress. The absolute max principal and signed von Mises methods can then be used to calculate the fatigue damage at any location in the fuse groove. The maximum value is then used as the final result for the analysis ${ }^{4}$. All of the properties necessary for a fatigue analysis including the cyclic stressstrain curve and Coffin-Manson fatigue constants were provided by UTAS-LS ${ }^{5}$.

Due to the premature failure of Pin A in test, the fatigue damages associated with this pin for all three fatigue analysis methods have been scaled to represent the damage accumulated at the time of failure. Conversely, the fatigue damages presented for Pin B and C are the fatigue damages predicted at the end of the test. For all three pins, the pure shear method severely over-predicts the fatigue damage in each of the three pins. Pin A is expected to have a value of 1 to indicate failure, while Pin B and C should all have damages below 1 since failure did not occur during the test.

\footnotetext{
${ }^{4}$ Due to the proprietary nature of this data, additional information regarding the calculation of these fatigue damages cannot be provided.

${ }^{5}$ Due to the proprietary nature of this data, the exact values for the material and fatigue properties cannot be provided.
} 
The absolute max principal and signed von Mises methods significantly under-predict the fatigue damage expected for Pin A, while over-predicting the damage for Pin B.

Table 6-3: Fatigue damages calculated by UTAS-LS for the three test fuse pins using three different fatigue analysis methodologies.

\begin{tabular}{|r|r|r|r|}
\hline & \multicolumn{3}{|c|}{ Fatigue Analysis Method } \\
\cline { 2 - 4 } & & $\begin{array}{r}\text { Absolute Max } \\
\text { Principal }\end{array}$ & $\begin{array}{r}\text { Signed Von } \\
\text { Mises }\end{array}$ \\
\hline Pin A $^{1}$ & 75.1 & 0.189 & 0.373 \\
\hline Pin B & 7.66 & 4.32 & 4.57 \\
\hline Pin C & 3.96 & 0.01 & 0.09 \\
\hline
\end{tabular}

Note: ${ }^{1}$ All fatigue damages scaled to represent damage accumulated at point of failure.

While the pure shear analysis provides no additional information regarding a predicted location of crack initiation, the other two methods do provide the analyst with a point of maximum fatigue damage. Both the absolute max principal and signed von Mises methods predict maximum fatigue damage at roughly the $-45^{\circ}$ location in the fuse pin for Pin A, the $-80^{\circ}$ location in the fuse pin for Pin $\mathrm{B}$, and the $25^{\circ}$ location in the fuse pin for Pin C. None of these analyses are able to predict the major crack initiation point at the neutral axis, which was found in the testing of Pin $\mathrm{A}$. The fatigue damage at the neutral axis for both the absolute max principal and signed von Mises methods were essentially zero for all three pins.

\section{Results using Multiaxial Stress-Strain Model and Fatigue Analysis Methods}

Fatigue analyses were performed at 13 different locations in each of the three test fuse pins using the methods outlined in Chapters 3, 4, and 5. For each analysis the notch correction method, cycle counting method, and fatigue damage criteria was varied. The fatigue damages were calculated using the following formula: 


$$
D=S F \times N_{b} \times \sum_{i=1}^{m} \frac{n_{i}}{N_{f}}
$$

where SF is the scatter factor used to account for variation in the fatigue life data, and $N_{b}$ is the number of blocks. The number of blocks represents the number of times the test spectrum is to be repeated to cover the expected life of the part being tested. The $n_{i}$ assumed a value of 0.5 for each reversal as counted by each cycle counting method and assumed a value of 1 for each full cycle counted. With this damage formulation, a damage value of 1 will indicate predicted failure in the test.

The results from the fatigue damage analyses are presented in Figure 6-22 through Figure 6-24 for Pin A, in Figure 6-25 through Figure 6-27 for Pin B, and in Figure 6-28 for Pin C. Only analyses that resulted in fatigue damages above a value of 0.1 were presented. The location of each analysis is indicated by the angular position $\left(0^{\circ}, \pm 45^{\circ}, \pm 90^{\circ}\right)$ and the percentage offset from the centre of the fuse groove. In each plot, the fatigue damage results have been grouped based on the cycle counting procedures. Note that the Bannantine-Socie method was analyzed using the shear strain component (E23) as a reference as this was considered to be the dominant stress and strain component in the fuse groove.

As expected, the fatigue analyses for Pin A resulted in the highest fatigue damages, with failure being predicted at the neutral axis by the Kandil-Brown-Miller (KBM) and the Liu Virtual Strain Energy (VSE) fatigue damage criteria with a number of combinations of cycle counting and notch correction methods. This correlates well with the test results which indicated very clear crack initiation at the neutral axis of Pin A. At the $45^{\circ}$ location, the analysis for Pin A at $19 \%$ offset to the centre of the fuse grove also predicts appreciable damage, but only the KBM criteria used with the Bannantine-Socie (BS) cycle counting procedure predicts failure. At this point in time, it is not clear whether any secondary cracks initiated at any location other than the neutral axis.

The analyses for Pin B where the KBM and Smith-Watson-Topper (SWT) fatigue damage criteria are used predict failure at the $-45^{\circ}$ and $-90^{\circ}$ locations in the fuse pin. This seems to follow the trend seen in the UTAS-LS uniaxial analyses and disagrees strongly with the fatigue test results, 
which resulted in no fatigue failure. The analyses for Pin C collectively predict no fatigue failure in the pin throughout the test load history, which is supported the experimental results. The effects of the fatigue damage criteria, notch correction method, and cycle counting procedures are discussed further in Section 6.5. 


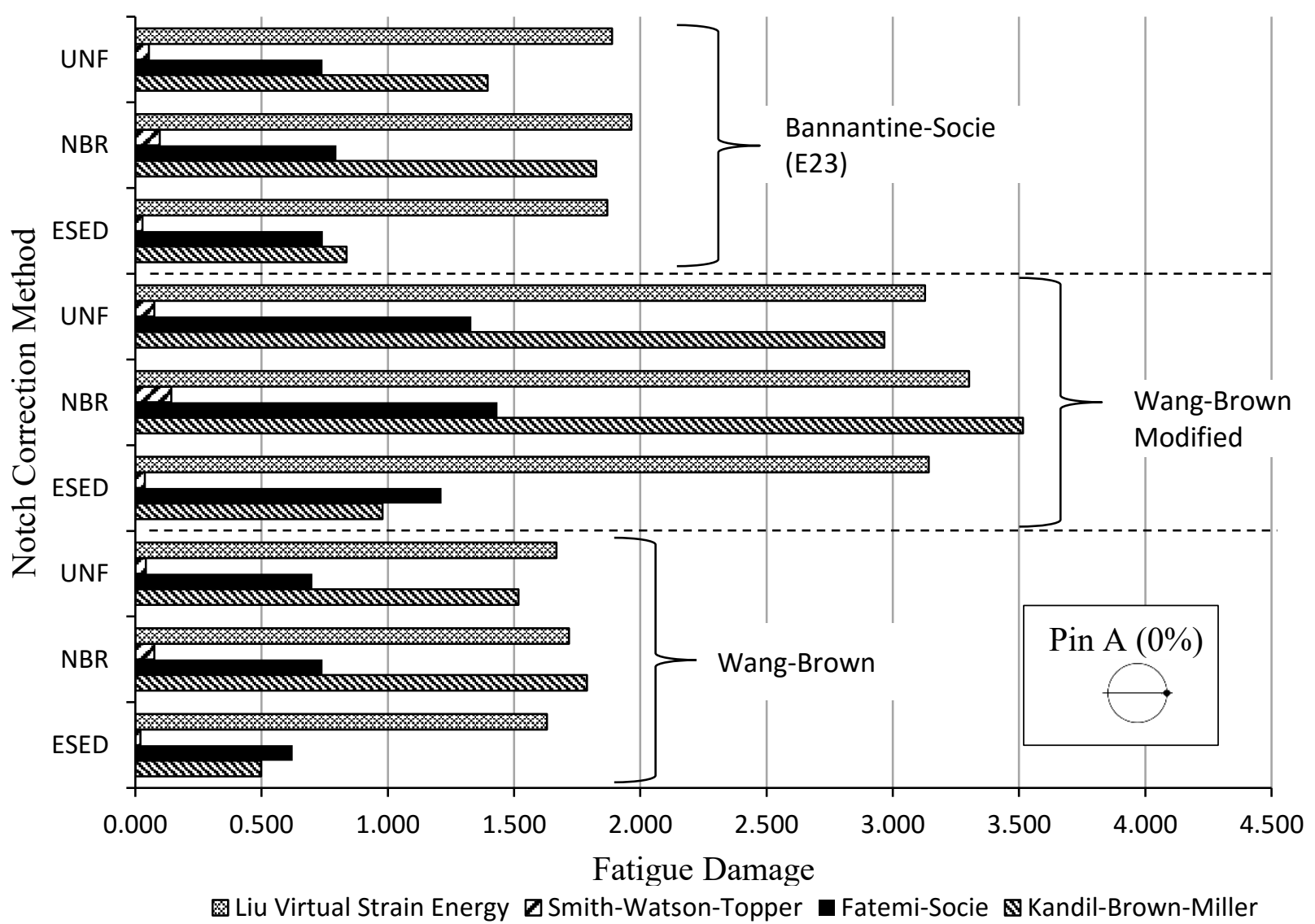

Figure 6-22: Fatigue damage predictions using FEA-based unit stresses for Pin A at the neutral axis and at the centre of the fuse groove. 


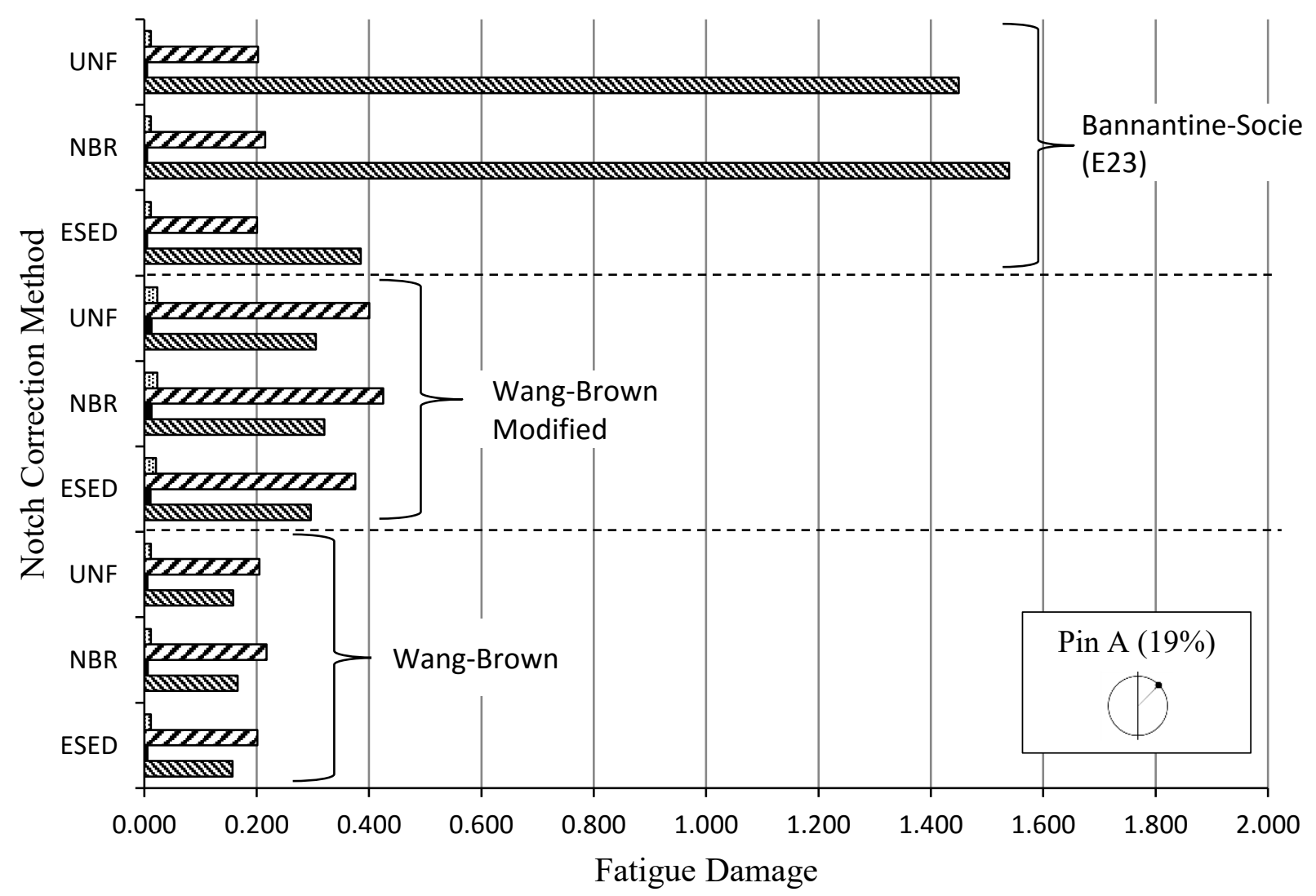

固 Liu Virtual Strain Energy $\square$ Smith-Watson-Topper $\square$ Fatemi-Socie $\mathbf{\$}$ Kandil-Brown-Miller

Figure 6-23: Fatigue damage predictions using FEA-based unit stresses for Pin $\mathrm{A}$ at $45^{\circ}$ to the neutral axis and at a 19\% offset from the centre of the fuse groove. 


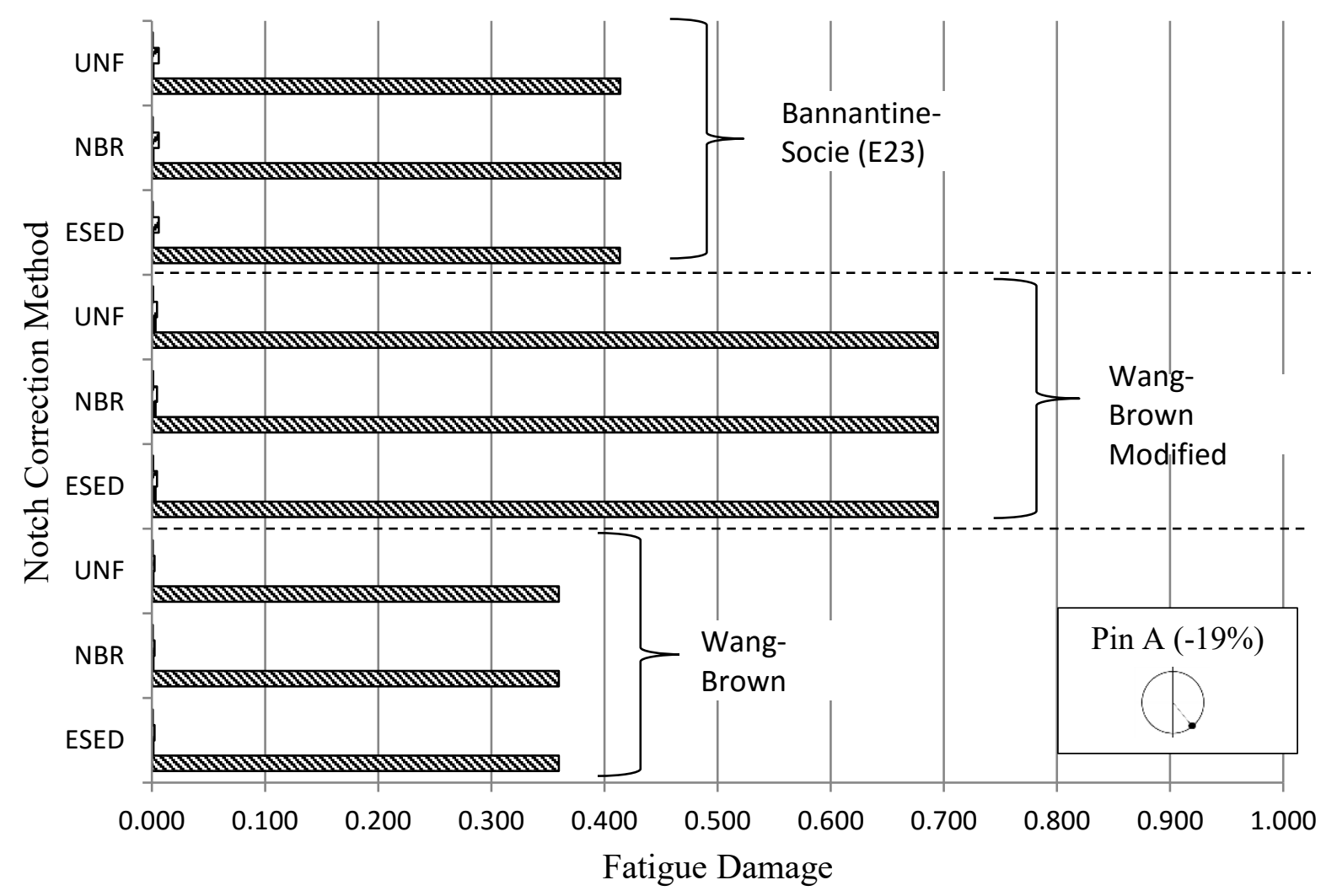

国 Liu Virtual Strain Energy $\square$ Smith-Watson-Topper Fatemi-Socie Kandil-Brown-Miller

Figure 6-24: Fatigue damage predictions using FEA-based unit stresses for Pin A at $-45^{\circ}$ to the neutral axis and at a $-19 \%$ offset from the centre of the fuse groove. 


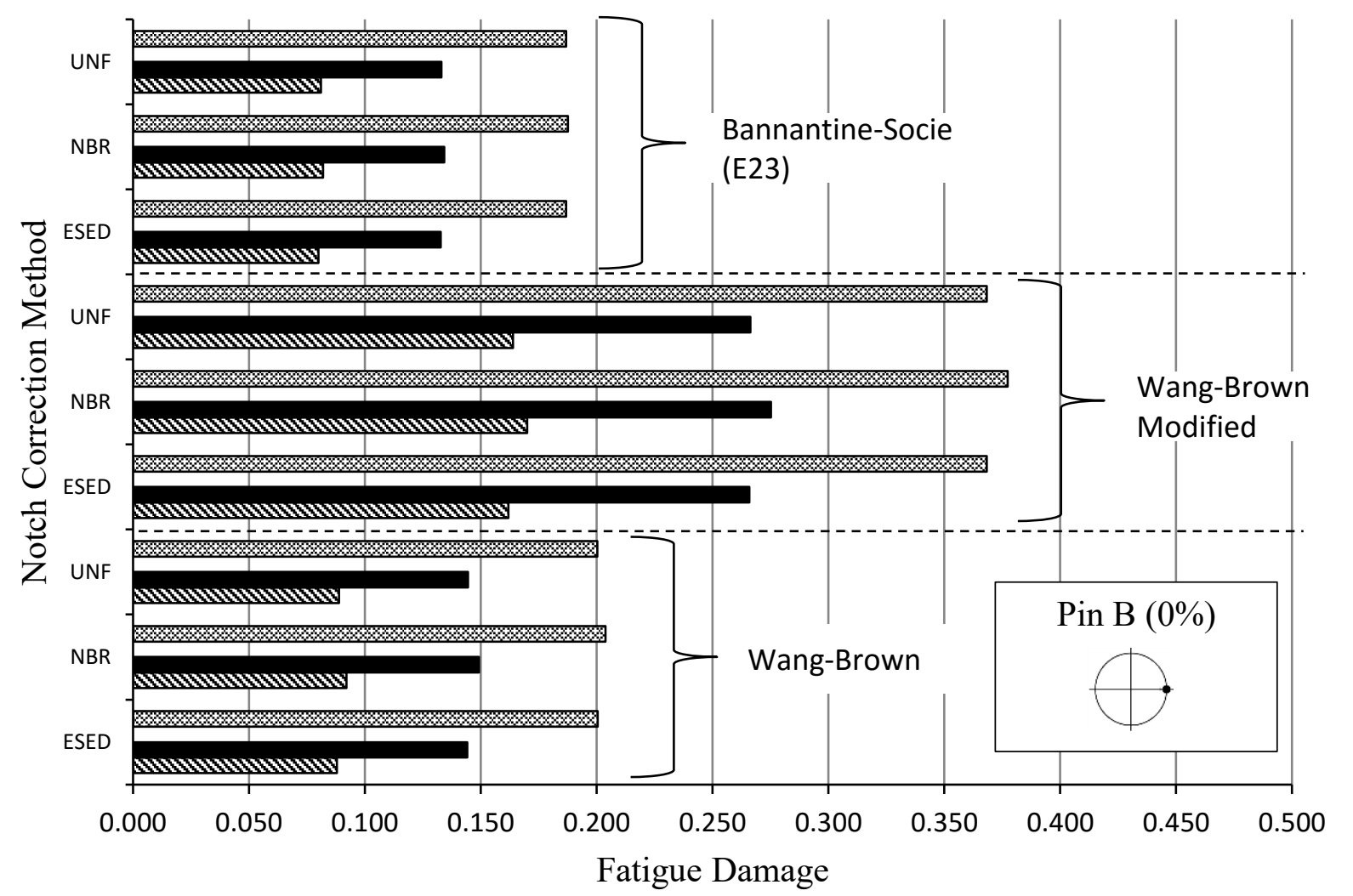

圈 Liu Virtual Strain Energy $\square$ Smith-Watson-Topper $\square$ Fatemi-Socie $\mathbf{s}$ Kandil-Brown-Miller

Figure 6-25: Fatigue damage predictions using FEA-based unit stresses for Pin B at the neutral axis and at the centre of the fuse groove. 


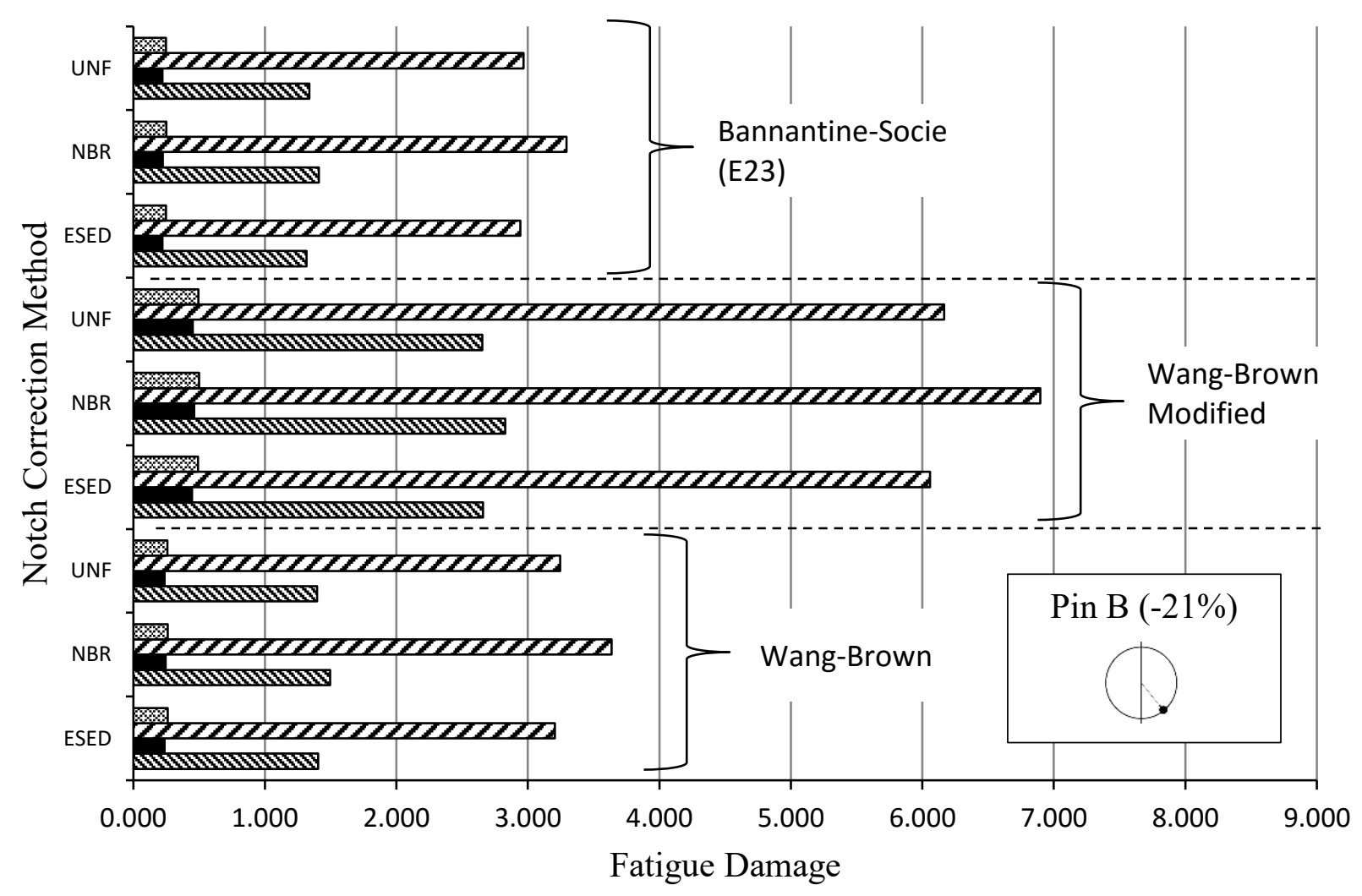

圈 Liu Virtual Strain Energy $\quad \square$ Smith-Watson-Topper $\quad$ Fatemi-Socie $\quad$ Kandil-Brown-Miller

Figure 6-26: Fatigue damage predictions using FEA-based unit stresses for Pin $\mathrm{B}$ at $-45^{\circ}$ to the neutral axis and at a $-21 \%$ offset to the centre of the fuse groove. 


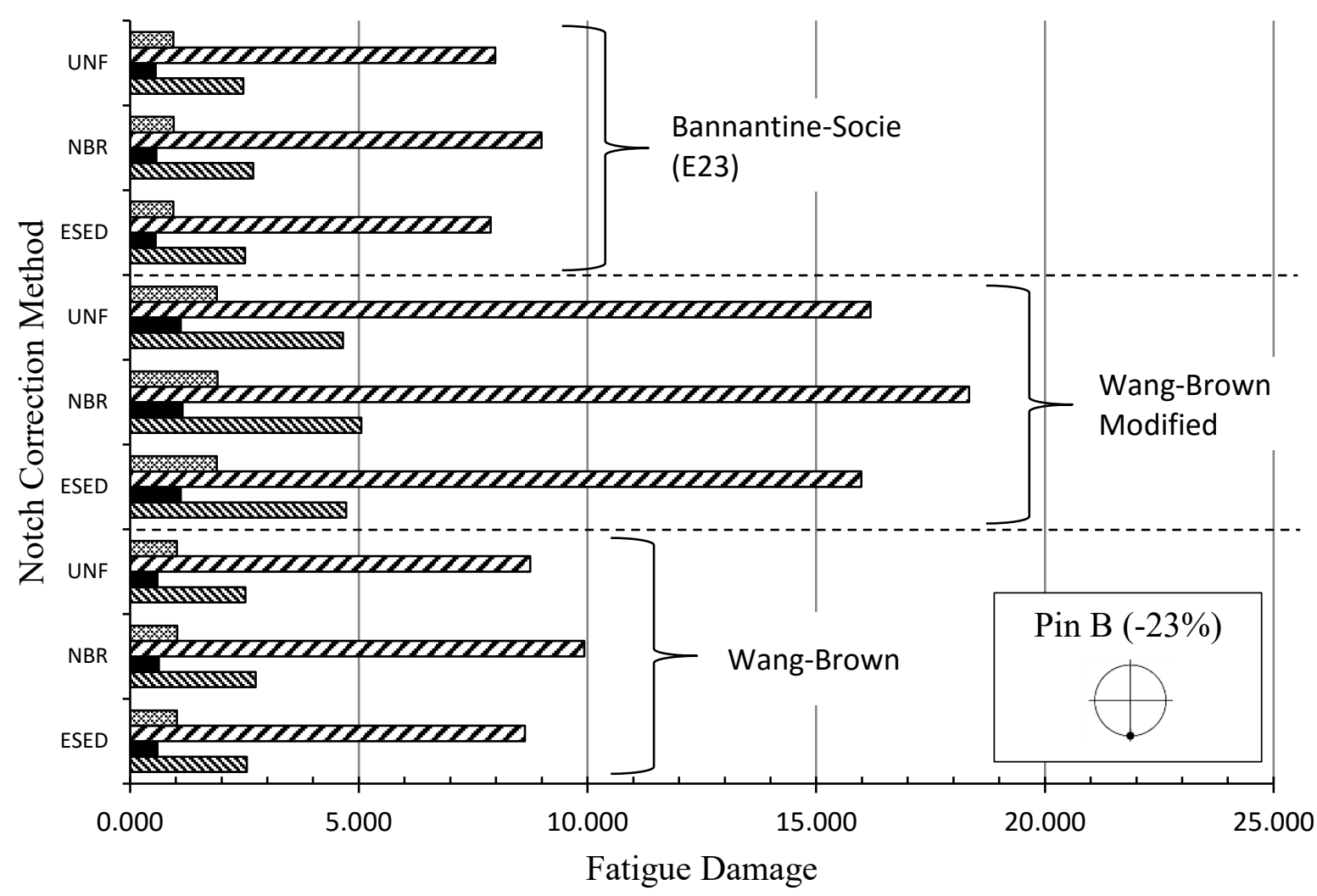

圆 Liu Virtual Strain Energy $\boldsymbol{Z}$ Smith-Watson-Topper $\mathbf{D a t e m i - S o c i e} \mathbf{\$}$ Kandil-Brown-Miller

Figure 6-27: Fatigue damage predictions using FEA-based unit stresses for Pin B at $-90^{\circ}$ to the neutral axis and at a $-23 \%$ offset to the centre of the fuse groove. 


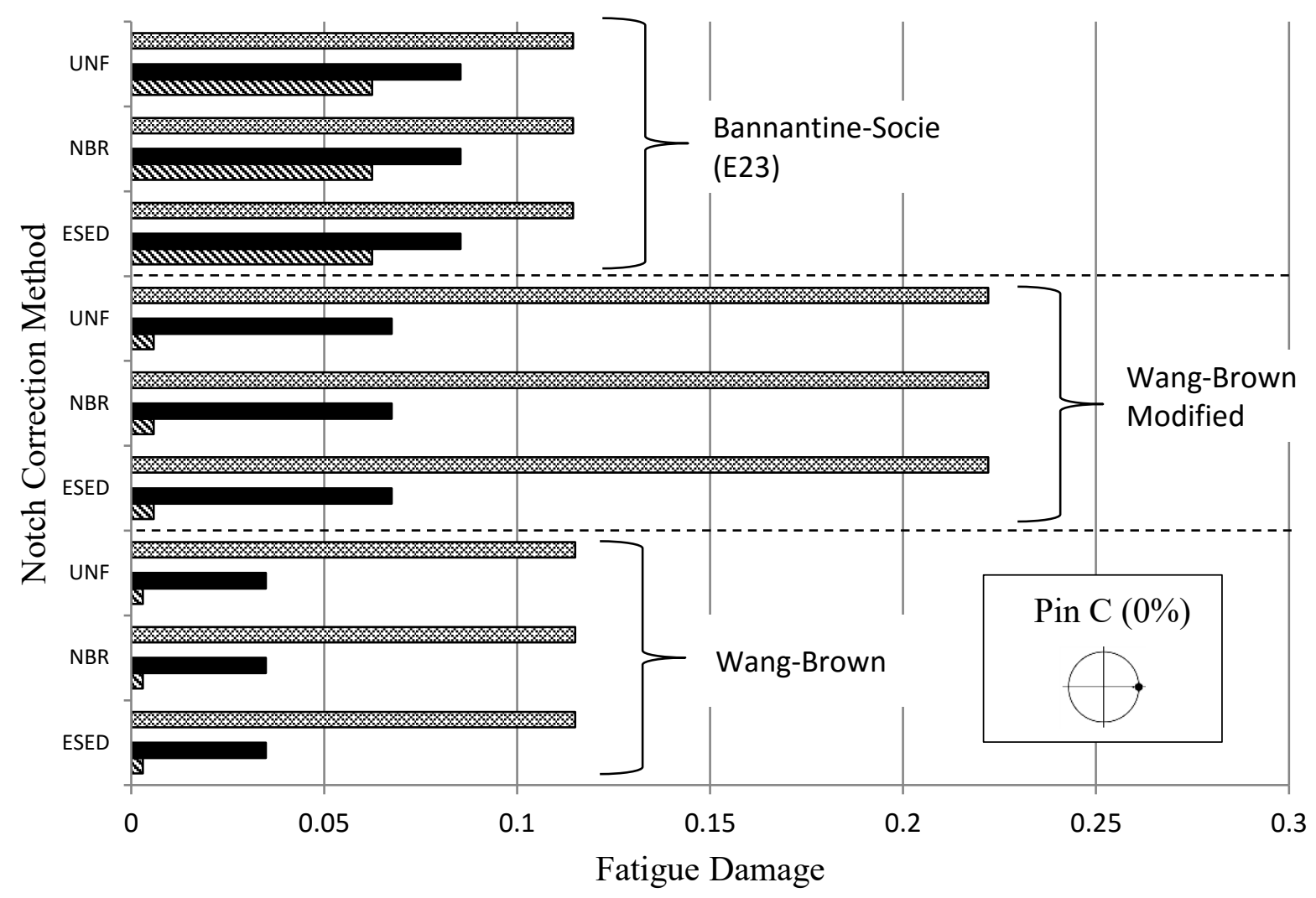

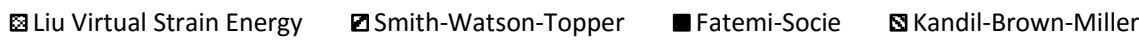

Figure 6-28: Fatigue damage predictions using FEA-based unit stresses for Pin $\mathrm{C}$ at the neutral axis and at centre of the fuse groove. 


\subsection{Discussion of Fatigue Life Predictions}

Having presented the fatigue damages using various combinations of fatigue damage criteria, notch correction methods, and cycle counting procedures in the previous section, the effects of each of these elements of the analysis procedure on the predicted damage is discussed in this section. In evaluating the performance of each of the elements of the fatigue analysis, the test results for Pin A at the neutral axis is used as the main test point for comparison since it is the only pin to have failed in test. Successful fatigue analyses are then those that are able to predict a fatigue failure with a fatigue damage of approximately 1 for Pin A. Fatigue analyses under-predicting damage in Pin A or predicting damages greater than a value of 1 for Pins $\mathrm{B}$ and $\mathrm{C}$ are then considered to be unsuccessful.

\subsubsection{Performance of Fatigue Criteria}

Due to the nature of each of the four fatigue damage criteria that were tested, there is a strong difference in the performance of each of the criteria. The KBM and FS model are both criteria that are based on the assumption of cracks initiating on maximum shear planes. These two models differ in that the KBM uses the normal strain while the FS criteria uses the maximum normal stress on the plane of maximum shear to capture the crack opening component of a given cycle. The SWT represents the class of fatigue damage criteria that assume that cracks will initiate on plane of maximum normal strain, and the Liu VSE criteria represents the class of energy-based fatigue damage criteria.

\section{Kandil-Brown-Miller (KBM) Fatigue Damage Criterion}

The KBM fatigue damage criterion overall predicts among the highest fatigue damage under all combinations of cycle counting procedures and notch correction methods compared to the other damage criteria. The KBM criterion still slightly under-predicts the fatigue damage in Pin A at the neutral axis, although this can be attributed to the under-predicted longitudinal strain as observed in Figure 6-7. This under-prediction is further supported by the under-predicted stress range based on the linear elastic FEA results seen in Figure 6-16. 
It is worth mentioning that the KBM criterion is the only criterion that results in appreciable damage at the $-45^{\circ}$ location in Pin A. While it is difficult to pinpoint the reason for this major difference between the KBM and the other three criteria, it may be argued that the KBM critiera is more sensitive to significant contributions of the normal strain component, even though it is based on the assumption of a max shear-initiated failure. This is supported by the dominant longitudinal stress range at the $-45^{\circ}$ location in Pin A that is comparable in magnitude to the shear stress range, while the max shear stress can reach up to $75 \%$ of the maximum shear stress at the neutral axis. It is difficult to say whether the KBM criterion produces the better estimate of damage at this location compared to the other criteria, since it is not certain whether or not cracks initiated at this location.

\section{Fatemi-Socie (FS) Fatigue Damage Criterion}

Overall the FS criterion performs similarly to the KBM criterion, except in the analysis of Pin B at the $-90^{\circ}$ location where the FS criterion is the only damage criterion that did not predict failure. The FS criterion is then the only criterion among the four fatigue damage criteria that did not result in any clearly overly-conservative damage predictions. Similar to the KBM criterion, the FS criterion generally under-predicts the fatigue damage of Pin A at the neutral axis.

\section{Smith-Watson-Topper (SWT) Fatigue Damage Criterion}

Analyses using the SWT fatigue damage criterion severely under-predicts the damage in Pin A at the neutral axis. This makes sense since the neutral axis is mostly loaded in shear and the SWT criterion is a normal strain-failure criterion. At other angular locations in Pin A the SWT criterion results in damage predictions that are comparable to that of analyses using the KBM and FS criteria. For Pin B the SWT criterion results in severe over-predictions of the fatigue damage at the $-45^{\circ}$ and the $-90^{\circ}$ locations, seen in Figure 6-26 and Figure 6-27. This would then further suggest that the low alloy steel used for these pins would tend to fail more in a shear mode than in an axial mode. Further material testing would need to be performed to verify the failure mode of the particular material used for these pins. 


\section{Liu Virtual Strain Energy (VSE) Fatigue Damage Criterion}

For Pin A at the neutral axis, the Liu VSE fatigue damage criterion results in reasonably conservative fatigue damages when used with the Bannantine-Socie and Wang-Brown cycle counting procedures. For Pin B and $\mathrm{C}$, this criterion produces generally good fatigue damage predictions, though it is clearly conservative in its prediction of the damages at the $-90^{\circ}$ location in Pin B as seen in Figure 6-27. Furthermore, for Pin B and C at the neutral axis the analyses using the Liu VSE criterion results in the highest fatigue damages among the other criteria, while still having damage values below a value of 1 . Based on the available test points, it can then be concluded that the Liu VSE fatigue damage criterion will generally provide conservative fatigue damage estimates in both shear stress- and axial stress-dominated stress states. This is can be very useful since the use of this criterion does not require the analyst to make a decision regarding the dominant failure mode when performing the fatigue analysis.

\subsubsection{Effect of Notch Correction Method}

The differences between the notch correction methods are best highlighted in Figure 6-22 for Pin A at the neutral axis, since this is the point where the most plasticity is observed. The analyses where the KBM fatigue damage criterion is used seem to be affected significantly by the choice of notch correction method, while analyses using the other damage criteria are affected rather minimally. In general, there is a small difference between the fatigue damages from analyses where the notch stresses and strains are calculated using the different notch correction methods. At the other locations, where plastic deformation is small, the difference between the three different notch correction methods is hardly noticeable. However large the difference in final fatigue damages, the ESED method consistently result in damage predictions on the lower end of the three notch correction methods, with Neuber producing the highest damages.

As discussed in Chapter 4, the ESED method is mathematically simpler and less prone to convergence issues. While this method is not capable of capturing the more complex plastic notch behaviours such as the coupling of axial and shear straining, the ESED method can provide reasonably reliable notch stresses and strains for the purpose of multiaxial fatigue analyses. The 
Neuber and Unified notch correction methods become more powerful when capturing the detailed strain components and complex cyclic behaviours such as ratchetting becomes more significant. Further testing may be required to determine the sensitivity of each of these methods to different degrees of stress concentration and material.

\subsubsection{Effect of Cycle Counting Procedure}

In general, there seems to be little difference in the final fatigue damages predicted by the WangBrown and Bannantine-Socie cycle counting procedures. The only instance where a significant difference between the results of the Wang-Brown and the Bannantine-Socie cycle counting procedures is observed is in Figure 6-23, at the $45^{\circ}$ location in Pin A, where the damages calculated with the KBM fatigue damage criteria differs by nearly an order of magnitude. This difference is isolated to analyses using the KBM criterion and thus suggests that it may be due to the combination of the Bannantine-Socie procedure with the KBM criterion rather than the cycle counting procedure on its own.

The Wang-Brown cycle counting procedure seems to operate quite predictably overall. As expected, the Wang-Brown Modified procedure essentially produces fatigue damages that are roughly double that of the Wang-Brown procedure due to the modified rules that allow the new procedure to evaluate both full cycles and reversals. The result of this modification appears to cause the Wang-Brown Modified procedure to severely over-predict the fatigue damage in all of the cases analyzed. It can then be concluded that the simple procedure of only identifying reversals, as per the original Wang-Brown procedure, is sufficient for producing reasonable fatigue damage predictions.

Based on the predicted fatigue damages, it is still not possible to say whether the Bannantine-Socie or the Wang-Brown cycle counting procedure performs better than the other since both procedures produce quite comparable results. This being said, due to the lack of user intervention required the Wang-Brown procedure can be considered to be a more practical choice when compared to the more involved Bannantine-Socie procedure, which requires the user to select a reference strain of significance and to select a number of critical planes upon which to perform a rainflow cycle count. 


\subsection{Conclusions}

The results of the fatigue analysis of the three test fuse pins provided by UTAS-LS were presented and significant improvements to the uniaxial fatigue analysis were demonstrated with the use of multiaxial fatigue analysis methodologies. Strains predicted using the Jiang-Sehitoglu kinematic hardening model were compared to the experimental strains measured at the neutral axis of each pin. Preliminary tests showed little difference between the results which included nonproportional hardening and the results that neglected non-proportional hardening. This was considered to be largely due to the small amount of plasticity encountered in the load histories being investigated. Results including non-proportional hardening were then neglected for the remainder of the fatigue analyses.

Large errors were observed in the hoop and longitudinal stress and strain predictions, particularly for Pin A. This was found to be due to the large amount of ratchetting that was not accounted for in the implementation of the multiaxial stress-strain model. The effect of these errors is reflected in the final fatigue damage predictions; however the strong correlation of the predicted shear strains to the experimental shear strains appears to be enough to provide reasonable damage predictions.

The test results of fuse pin A were used to determine how well each combination of fatigue damage criterion, notch correction method, and cycle counting procedure could predict a known fatigue failure. The test results of fuse pins $\mathrm{B}$ and $\mathrm{C}$ were used to highlight overly conservative combinations of fatigue damage criterion, notch correction method, and cycle counting procedure. The SWT damage criterion was found to severely under-predict the damage of pin A that the neutral axis under all combinations of notch correction method and cycle counting procedure and was overly conservative for the Pin B and C analyses. The KBM, FS, and Liu VSE fatigue damage criteria provided generally good estimations of the damage in Pin A, with Liu VSE being the most conservative. The FS criterion was found to be better than the KBM criterion in that it was less likely to be result in overly conservative results where damage is not expected. The selection of cycle counting procedures and notch correction method was found to not play a significant role in the final fatigue damages compared to the choice of fatigue damage criterion and stress-strain 
model. Out of the four elements of the fatigue analysis process, it is concluded that improvements in the multiaxial stress-strain model will have the most impact on the final fatigue life predictions. 


\section{Chapter 7 : Conclusions and Recommendations}

The objective of this research was to investigate the use of different multiaxial fatigue analysis methods to improve the fatigue life prediction of aircraft landing gear fuse pins. Experimental results from the literature was used to verify the design and implementation of a complete fatigue analysis program that includes the multiaxial stress-strain model, notch correction calculations, cycle counting procedures, and fatigue damage calculations proposed in literature. Test data provided by UTAS-LS was used to then compare the applicability of each combination of fatigue analysis procedures in predicting the fatigue life of three fuse pin designs. This chapter summarizes the key conclusions and recommendations.

\subsection{Conclusions}

\section{Verification of the fatigue analysis program}

For smooth specimens, nearly perfect correlation was found for monotonic and cyclic load histories following proportional and non-proportional stress paths. This demonstrated the basic implementation of the Jiang-Sehitoglu kinematic hardening model and the use of Tanaka nonproportional hardening coefficient.

The program was further tested for notched specimens where the notch stresses and strains were calculated using three different notch correction methods: the incremental ESED method, the multiaxial Neuber method, and the Unified method. Specimens with varying stress concentration factors and varying ductility were used to determine the sensitivity of the notch correction methods to the geometry of the notch and the material properties. It was found that the three methods performed similarly for proportional loading histories, and all three performed best for high stress concentration factor geometries and low ductility materials. The notch correction methods were also tested for non-proportional loading histories and it was found that predictions would tend to under-predict shear strains by up to $30 \%$ and axial strains by up to $50 \%$. Contrary to observations in the literature, the current implementation of the notch correction methods seems unable to 
capture the axial-shear coupling in the non-proportional loading histories, which is the main reason for the under-predictions of the strains.

The Wang-Brown and Bannantine-Socie multiaxial cycle counting procedures were briefly tested to ensure that cycle counts operated as expected. A modified Wang-Brown procedure was proposed that includes a step for distinguishing between full cycles and half-cycles in a manner similar to the uniaxial rainflow cycle counting procedure. This was included as an attempt to have a modified version of Wang-Brown that produced the same results as the rainflow cycle counting procedure for uniaxial cases. Examples from the ASTM E1049-85 standard for cycle counting and the literature were used as benchmark tests.

\section{Analysis of the Test Fuse Pins}

Multiaxial fatigue analyses were performed for three different fuse pins and the results were compared to the results from tests performed by UTAS-LS. The notch correction method, cycle counting procedure, and fatigue damage criterion was varied for each fatigue analysis. At the load level that was analyzed, which was selected to match the test load history, no significant nonproportional hardening effect was observed.

The effect of four different fatigue damage criterion on the final fatigue damage prediction was investigated. The KBM and FS damage criteria, both assuming shear-dominated crack initiation, performed reasonably well. The KBM was more conservative than the FS criterion and was found to be over-conservative in predicting the fatigue failure where it was not found to occur in test. The SWT damage criterion, which assumes axial-dominated crack initiation, performed poorly in most scenarios. The SWT criterion under-predicted expected fatigue failure and over-predicted damage in areas where no failure was expected. The Liu VSE damage criterion, the energy-based criterion, was a good generalized method that performed equally well for axial- and sheardominant stress states. The Liu VSE damage criterion was found to be generally more conservative than the KBM and FS damage criteria. Based on the results of this investigation, the Liu VSE damage criterion is considered to be a good choice when the analyst is uncertain of the 
nature of crack initiation for the material under consideration. When cracks are expected to initiate due to shear stresses or shear strains, the FS damage criterion is found to be a suitable choice.

Other than the damage criterion selection, the notch correction method and cycle counting procedure selection was found to have less effect on the final fatigue damage prediction. The Wang-Brown Modified procedure, which was proposed in this thesis, was found to be overconservative in all scenarios. It is thus concluded that the original Wang-Brown procedure, where only reversals are counted, and the Bannantine-Socie procedure, which requires a rainflow cycle count to be performed on selected critical planes for a chosen reference strain component, are both suitable choices. Overall, it was found that the performance of the stress-strain model and the selection of the fatigue damage criterion had the most effect on the final fatigue life prediction of the fuse pins analyzed in this thesis.

\subsection{Recommendations}

A major component of the fatigue analysis procedure that can be improved upon is the stress-strain model. Relative success in using other hardening models such as the Mroz kinematic hardening model and the Garud hardening model has been reported in the literature. These models could be implemented and compared directly to the Jiang-Sehitoglu model in future iterations of this work.

While the Jiang-Sehitoglu model also has been reported to work very well, many of the calculations in the model have been simplified for the scope of this work. Additional work to include the more advanced components of the Jiang-Sehitoglu model, such as ratchetting and material memory, can be investigated in the future. This would require additional material tests that would allow for the comprehensive calculation of the material constants in the Jiang-Sehtiglu model as originally proposed in the literature instead of the simplified approximations used in this thesis.

The notch correction methods could also be investigated further to determine sensitivities to shearand axial-dominated stress and strain histories. This can potentially help to provide users and researchers with a better understanding of the capabilities and limitations of the ESED, Neuber, 
and Unified methods. The cycle counting procedures can also be tested for more complex stress and strain histories; more non-proportional histories may show a more significant difference between the Wang-Brown procedure, the Bannantine-Socie procedure, and the proposed modified Wang-Brown procedure. Additional fatigue damage criteria beyond the four that were tested in this thesis can also be investigated in the future.

Finally, additional testing of fuse pins to the point of failure would provide more test points for the verification and correlation of the proposed multiaxial fatigue analysis methods. At this point in time, only one of the three fuse pins have been tested to failure and thus all observations and conclusions rely on this single test point. Furthermore, in future tests strain gauges should also be placed at positions that are offset to the centre of the fuse groove to capture the larger longitudinal strain cycles that are not present at the centre of the fuse groove.

\subsection{Contributions}

The results of the research presented demonstrates the potential for significant improvement of fatigue life predictions of landing gear fuse pins by implementing multiaxial fatigue methods. With future successful use of the methods presented in this thesis, practicing engineers performing fatigue analyses will be able to certify more efficient designs for service. For customers, such as the aircraft manufacturers and airlines, significant savings will be seen in the long-term maintenance costs of the landing gear assembly since the fuse pins will no longer be life limited due to limitations in the fatigue analysis of the pins.

\subsection{Scope and Limitations}

The results of this research should be treated as preliminary at this stage. Successful implementation of the multiaxial fatigue methods has been demonstrated for the three fuse pins provided by UTAS. It is important to note that the results of this research is based on a single test point since only one of the three fuse pins failed in test; therefore, to make any further use of the conclusions of this work additional testing where the fuse pins are tested to failure is required. 


\section{Bibliography}

Anderson, T. L. (2005). Fracture Mechanics: Fundamentals and Applications (3rd ed.). CRC Press.

ASTM International. (2011). Standard Practices for Cycle Counting in Fatigue Analysis. West Conshohocken, PA.

Bannantine, J. A., \& Socie, D. F. (1989). A Variable Amplitude Multiaxial Fatigue Life Prediction Method. Third International Conference on Biaxial/Multiaxial Fatigue. Stuttgart.

Bannantine, J. A., Comer, J. J., \& Handrock, J. L. (1990). Fundamentals of Metal Fatigue Analysis. Englewood Cliffs: Prentice Hall.

Barkey, M. E. (1993). Calculation of notch strains under multiaxial nominal loading. PhD thesis, University of Illinois, Urbana-Champaign.

Callister, W. D., \& Rethwish, D. G. (2010). Materials Science and Engineering: An Introduction (8th ed.). U.S.A.: John Wiley \& Sons, Inc.

Dowling, N. E. (1972, March). Fatigue Failure Predictinos for Complicated Stress-Strain Histories. Journal of Materials, 7(1), 71-87.

Dowling, N. E. (1993). Mechanical Behavior of Materials. Englewood Cliffs, New Jersey: Prentice-Hall, Inc.

Fatemi, A., \& Shamsaei, N. (2011). Multiaxial fatigue: An overview and some approximation models for life estimation. International Journal of Fatigue, 33, 948-958. 
Fatemi, A., \& Yang, L. (1998). Cumulative fatigue damage and life prediction theories: a survey of the state of the art for homogeneous materials. International Journal of Fatigue, 20(1), 9-34.

Firat, M. (2011). A notch strain calculation of a notched specimen under axial-torsion loadings. Materials and Design, 32, 3876-3882.

Frederick, C. O., \& Armstrong, P. (1966). A mathematical representation of the multiaxial Bauschinger effect. Materials at High Temperatures, 24(1), 11-26.

Fuchs, H. O., \& Stephens, R. I. (1980). Metal Fatigue in Engineering. John Wiley \& Sons , Inc.

Garud, Y. (1981). A new approach to the evaluation of fatigue under multiaxial loadings. Journal of Engineering Materials and Technology, 118-123.

Gates, N., \& Fatemi, A. (2014). Notched fatigue behavior and stress analysis under multiaxial states of stress. International Journal of Fatigue, 67, 2-14.

Ince, A., Glinka, G., \& Buczynski, A. (2014). Computational modeling of multiaxial elasto-plastic stress-strain response for notched components under nonproportional loading. International Journal of Fatigue, 62, 42-52.

Jiang, Y. Y., \& Kurath, P. (1996). Characteristics of the Armstrong-Frederick type plasticity models. International Journal of Plasticity, 12(3), 387-415.

Jiang, Y., \& Sehitoglu, H. (1996a). Modeling of Cycic Ratchetting Plasticity, Part I: Development of Constitutive Relations. Journal of Applied Mechanics, 63(3), 720725.

Jiang, Y., \& Sehitoglu, H. (1996b). Modeling of Cyclic Ratchetting Plasticity, Part II: Comparison of Model Simulations with Experiments. Journal of Applied Mechanics, 63(3), 726-733. 
Kanazawa, K., Miller, K., \& Brown, M. (1979). Cyclic Deformation of 1\% Cr-Mo-V Under Out-of-Phase Loads. Fatigue of Engineering Materials and Structures, 217228.

Kandil, F. A., Brown, M. W., \& Miller, K. J. (1981). Biaxial low-cycle fatigue failure of 316 stainless steel at elevated temperatures. International Conference on Mechanical Behaviour and Nuclear Applications of Stainless Steel at Elevated Temperatures.

Kim, K. S., \& Park, J. C. (1999). Shear strain based multiaxial fatigue parameters applied to variable amplitude loading. International Journal of Fatigue, 21, 475-483.

Kottgen, V. B., Barkey, M. E., \& Socie, D. F. (1995). Pseudo Stress and Pseudo Strain Based Approaches to Multiaxial Notch Analysis. Fatigue Fract. Engng Mater. Struct., 18(9), 981-1006.

Li, B., Reis, L., \& de Freitas, M. (2009). Comparative study of multiaxial fatigue damage models for ductile structural steels and brittle materials. Internationaly Journal of Fatigue, 1895-1906.

Liu, K. C. (1993). A Method Based on Virtual Strain-Energy Parameters for Multaxial Fatigue Life Prediction. Advances in Multiaxial Fatigue, STP 1191, pp. 67-84.

Marquis, G. B., \& Socie, D. F. (2000). Multiaxial Fatigue. Warrendale, U.S.A.: Society of Automotive Engineers, Inc.

Meggiolaro, M. A., \& Pinho de Castro, J. T. (2014). Prediction of non-proportionality factors of multiaxial histories using the Moment Of Inertia method. International Journal of Fatigue, 61, 151-159.

Mroz, Z. (1967). On the description of anisotropic workhardening. Journal of the Mechanics of Physics of Solids, 163-175. 
Narayan, K., Behdinan, K., \& Vanderpol, P. (2006). An equivalent uniaxial fatigue stress model for analyzing landing gear fuse pins. Strength of Materials, 38(3).

Shamsaei, N., \& Fatemi, A. (2009). Effect of hardness on multiaxial fatigue behaviour and some simple approximations for steels. Fatigue \& Fracture of Engineering Materials \& Structures, 32(8), 631-646.

Shamsaei, N., \& Fatemi, A. (2010). Effect of microstructure and hardness on nonproportional cyclic hardening coefficient and predictions. Materials Science and Engineering, 527(12), 3015-3024.

Shamsaei, N., Fatemi, A., \& Socie, D. F. (2010). Multiaxial cyclic deformation and nonproportional hardening employing discriminating load paths. International Journal of Plasticity, 1680-1701.

Shamsaei, N., Fatemi, A., \& Socie, D. F. (2011). Multiaxial fatigue evaluation using discriminating strain paths. International Journal of Fatigue, 33, 567-609.

Singh, M. N., Glinka, G., \& Dubey, R. N. (1996). Elastic-plastic stress-strain calculation in notched bodies subjected to non-proportional loading. International Journal of Fracture, 76, 39-60.

Smith, R. N., Watson, P., \& Topper, T. H. (1970). A Stress-Strain Parameter for the Fatigue of Metals. Journal of Materials, 5(4), 767-778.

Socie, D. F. (n.d.). Retrieved from eFatigue: Multiaxial Fatigue: https://www.efatigue.com/multiaxial/background/strainlife.html\#Plasticity

Socie, D. F., \& Marquis, G. B. (2000). Multiaxial Fatigue. Warrendale, PA: Society of Automotive Engineers, Inc.

Suresh, S. (1998). Fatigue of Materials. New York, U.S.A.: Cambridge University Press. 
Tanaka, E. (1994). A nonproportionality parameter and a cyclic viscoplastic constitutive model taking into account amplitude dependences and memory effects of isotropic hardening. European Journal of Mechanics, 13(2), 155-173.

Tipton, S. M., \& Bannantine, J. A. (1993). Inelastic Stress-Strain Predictions for Multiaxial Fatigue Damage Evaluation. Advances in Multiaxial Fatigue, ASTM STP 1191, pp. 273-297.

Wang, C. H., \& Brown, M. W. (1996, July). Life Prediction Techniques for Variable Amplitude Multiaxial Fatigue - Part 1: Theories. Journal of Engineering Materials and Technology, 118(2), 367-370.

Wang, Y.-Y., \& Yao, W.-X. (2004). Evaluation and comparison of several multiaxial fatigue criteria. International Journal of Fatigue, 26, 17-25.

Wu, Z.-R., Hu, X.-T., \& Song, Y.-D. (2014). Multiaxial fatigue life prediction for titanium alloy TC4 under proportional and nonproportional loading. International Journal of Fatigue, 59, 170-75.

Ye, D., Hertel, O., \& Vormwald, M. (2008). A unified expression of elastic-plastic notch stress-strain calculation in bodies subjected to multiaxial cyclic loading. International Journal of Solids and Structures, 45, 6177-6189.

Yokobori, Y., Yamanouchi, H., \& Yamamoto, S. (1965). Low Cycle Fatigue of ThinWalled Hollow Cylinder Specimens of Mild Steel in Uniaxial and Torsional Tests at Constant Amplitude. International Journal of Fracture, 1(1), 3-13.

Zhang, J., \& Jiang, Y. (2008). Constitutive modeling of cyclic plasticity deformation of a pure polycrystalline copper. International Journal of Plasticity, 24, 1890-1915. 


\section{Appendices}

\section{Appendix A Fatigue Damage Results Calculated using Multiaxial Fatigue Approach}

This appendix presents all of the fatigue damage results calculated using the multiaxial fatigue methodologies presented in the main body of this thesis. The results of the analysis of the three test fuse pins are presented for 13 locations in the fuse groove of each of these pins. 


\section{A.1 Pin A FEA-Based Fatigue Damage Results}

Table A.1-1: Fatigue damage results for Pin A at the neutral axis and centre of the fuse groove $(\hat{\mathrm{z}}=0)$.

\begin{tabular}{|c|c|c|c|}
\hline Notch Method & Cycle Count & Fatigue Criterion & Damage \\
\hline ESED & Wang-Brown & KBM & 0.4983 \\
\hline NBR & Wang-Brown & KBM & 1.7885 \\
\hline UNF & Wang-Brown & KBM & 1.5178 \\
\hline ESED & Wang-Brown Modified & KBM & 0.9791 \\
\hline NBR & Wang-Brown Modified & KBM & 3.5155 \\
\hline UNF & Wang-Brown Modified & KBM & 2.9661 \\
\hline ESED & Bannantine-Socie & KBM & 0.8363 \\
\hline NBR & Bannantine-Socie & KBM & 1.8247 \\
\hline UNF & Bannantine-Socie & KBM & 1.3947 \\
\hline ESED & Wang-Brown & FS & 0.6236 \\
\hline NBR & Wang-Brown & FS & 0.7419 \\
\hline UNF & Wang-Brown & FS & 0.7008 \\
\hline ESED & Wang-Brown Modified & FS & 1.2121 \\
\hline NBR & Wang-Brown Modified & FS & 1.4344 \\
\hline UNF & Wang-Brown Modified & FS & 1.3305 \\
\hline ESED & Bannantine-Socie & FS & 0.7425 \\
\hline NBR & Bannantine-Socie & FS & 0.7967 \\
\hline UNF & Bannantine-Socie & FS & 0.7406 \\
\hline ESED & Wang-Brown & SWT & 0.0198 \\
\hline NBR & Wang-Brown & SWT & 0.075 \\
\hline UNF & Wang-Brown & SWT & 0.0422 \\
\hline ESED & Wang-Brown Modified & SWT & 0.0372 \\
\hline NBR & Wang-Brown Modified & SWT & 0.142 \\
\hline UNF & Wang-Brown Modified & SWT & 0.0757 \\
\hline ESED & Bannantine-Socie & SWT & 0.0282 \\
\hline NBR & Bannantine-Socie & SWT & 0.0962 \\
\hline UNF & Bannantine-Socie & SWT & 0.0536 \\
\hline ESED & Wang-Brown & Liu VSE & 1.6308 \\
\hline NBR & Wang-Brown & Liu VSE & 1.7174 \\
\hline UNF & Wang-Brown & Liu VSE & 1.6674 \\
\hline ESED & Wang-Brown Modified & Liu VSE & 3.1425 \\
\hline NBR & Wang-Brown Modified & Liu VSE & 3.302 \\
\hline UNF & Wang-Brown Modified & Liu VSE & 3.1275 \\
\hline ESED & Bannantine-Socie & Liu VSE & 1.8698 \\
\hline NBR & Bannantine-Socie & Liu VSE & 1.9651 \\
\hline UNF & Bannantine-Socie & Liu VSE & 1.888 \\
\hline
\end{tabular}


Table A.1-2: Fatigue damage results for Pin A at $45^{\circ}$ to the neutral axis and centre of the fuse groove $(\hat{z}=0)$.

\begin{tabular}{|c|c|c|c|}
\hline Notch Method & Cycle Count & Fatigue Criterion & Damage \\
\hline ESED & Wang-Brown & KBM & 0.000 \\
\hline NBR & Wang-Brown & KBM & 0.000 \\
\hline UNF & Wang-Brown & KBM & 0.000 \\
\hline ESED & Wang-Brown Modified & KBM & 0.001 \\
\hline NBR & Wang-Brown Modified & KBM & 0.001 \\
\hline UNF & Wang-Brown Modified & KBM & 0.001 \\
\hline ESED & Bannantine-Socie & KBM & 0.059 \\
\hline NBR & Bannantine-Socie & KBM & 0.059 \\
\hline UNF & Bannantine-Socie & KBM & 0.059 \\
\hline ESED & Wang-Brown & $\mathrm{FS}$ & 0.003 \\
\hline NBR & Wang-Brown & $\mathrm{FS}$ & 0.003 \\
\hline UNF & Wang-Brown & FS & 0.003 \\
\hline ESED & Wang-Brown Modified & FS & 0.006 \\
\hline NBR & Wang-Brown Modified & FS & 0.006 \\
\hline UNF & Wang-Brown Modified & FS & 0.006 \\
\hline ESED & Bannantine-Socie & FS & 0.009 \\
\hline NBR & Bannantine-Socie & FS & 0.009 \\
\hline UNF & Bannantine-Socie & FS & 0.009 \\
\hline ESED & Wang-Brown & SWT & 0.006 \\
\hline NBR & Wang-Brown & SWT & 0.006 \\
\hline UNF & Wang-Brown & SWT & 0.006 \\
\hline ESED & Wang-Brown Modified & SWT & 0.010 \\
\hline NBR & Wang-Brown Modified & SWT & 0.010 \\
\hline UNF & Wang-Brown Modified & SWT & 0.010 \\
\hline ESED & Bannantine-Socie & SWT & 0.005 \\
\hline NBR & Bannantine-Socie & SWT & 0.005 \\
\hline UNF & Bannantine-Socie & SWT & 0.005 \\
\hline ESED & Wang-Brown & Liu VSE & 0.008 \\
\hline NBR & Wang-Brown & Liu VSE & 0.008 \\
\hline UNF & Wang-Brown & Liu VSE & 0.008 \\
\hline ESED & Wang-Brown Modified & Liu VSE & 0.015 \\
\hline NBR & Wang-Brown Modified & Liu VSE & 0.015 \\
\hline UNF & Wang-Brown Modified & Liu VSE & 0.015 \\
\hline ESED & Bannantine-Socie & Liu VSE & 0.009 \\
\hline NBR & Bannantine-Socie & Liu VSE & 0.009 \\
\hline UNF & Bannantine-Socie & Liu VSE & 0.009 \\
\hline
\end{tabular}


Table A.1-3: Fatigue damage results for Pin A at $45^{\circ}$ to the neutral axis and offset $19 \%$ from the centre of the fuse groove $(\hat{z}=0.19)$.

\begin{tabular}{|c|c|c|c|}
\hline Notch Method & Cycle Count & Fatigue Criterion & Damage \\
\hline ESED & Wang-Brown & KBM & 0.157 \\
\hline NBR & Wang-Brown & KBM & 0.166 \\
\hline UNF & Wang-Brown & KBM & 0.158 \\
\hline ESED & Wang-Brown Modified & KBM & 0.296 \\
\hline NBR & Wang-Brown Modified & KBM & 0.320 \\
\hline UNF & Wang-Brown Modified & KBM & 0.305 \\
\hline ESED & Bannantine-Socie & KBM & 0.385 \\
\hline NBR & Bannantine-Socie & KBM & 1.539 \\
\hline UNF & Bannantine-Socie & KBM & 1.450 \\
\hline ESED & Wang-Brown & FS & 0.007 \\
\hline NBR & Wang-Brown & FS & 0.008 \\
\hline UNF & Wang-Brown & FS & 0.008 \\
\hline ESED & Wang-Brown Modified & FS & 0.013 \\
\hline NBR & Wang-Brown Modified & FS & 0.015 \\
\hline UNF & Wang-Brown Modified & FS & 0.015 \\
\hline ESED & Bannantine-Socie & FS & 0.007 \\
\hline NBR & Bannantine-Socie & FS & 0.007 \\
\hline UNF & Bannantine-Socie & FS & 0.007 \\
\hline ESED & Wang-Brown & SWT & 0.201 \\
\hline NBR & Wang-Brown & SWT & 0.218 \\
\hline UNF & Wang-Brown & SWT & 0.205 \\
\hline ESED & Wang-Brown Modified & SWT & 0.376 \\
\hline NBR & Wang-Brown Modified & SWT & 0.425 \\
\hline UNF & Wang-Brown Modified & SWT & 0.400 \\
\hline ESED & Bannantine-Socie & SWT & 0.200 \\
\hline NBR & Bannantine-Socie & SWT & 0.215 \\
\hline UNF & Bannantine-Socie & SWT & 0.203 \\
\hline ESED & Wang-Brown & Liu VSE & 0.012 \\
\hline NBR & Wang-Brown & Liu VSE & 0.012 \\
\hline UNF & Wang-Brown & Liu VSE & 0.012 \\
\hline ESED & Wang-Brown Modified & Liu VSE & 0.021 \\
\hline NBR & Wang-Brown Modified & Liu VSE & 0.023 \\
\hline UNF & Wang-Brown Modified & Liu VSE & 0.023 \\
\hline ESED & Bannantine-Socie & Liu VSE & 0.012 \\
\hline NBR & Bannantine-Socie & Liu VSE & 0.012 \\
\hline UNF & Bannantine-Socie & Liu VSE & 0.012 \\
\hline
\end{tabular}


Table A.1-4: Fatigue damage results for Pin A at $45^{\circ}$ to the neutral axis and offset $-19 \%$ from the centre of the fuse groove $(\hat{z}=-0.19)$.

\begin{tabular}{|c|c|c|c|}
\hline Notch Method & Cycle Count & Fatigue Criterion & Damage \\
\hline ESED & Wang-Brown & KBM & 0.002 \\
\hline NBR & Wang-Brown & KBM & 0.002 \\
\hline UNF & Wang-Brown & KBM & 0.002 \\
\hline ESED & Wang-Brown Modified & KBM & 0.004 \\
\hline NBR & Wang-Brown Modified & KBM & 0.004 \\
\hline UNF & Wang-Brown Modified & KBM & 0.004 \\
\hline ESED & Bannantine-Socie & KBM & 0.004 \\
\hline NBR & Bannantine-Socie & KBM & 0.004 \\
\hline UNF & Bannantine-Socie & KBM & 0.004 \\
\hline ESED & Wang-Brown & $\mathrm{FS}$ & 0.002 \\
\hline NBR & Wang-Brown & $\mathrm{FS}$ & 0.002 \\
\hline UNF & Wang-Brown & FS & 0.002 \\
\hline ESED & Wang-Brown Modified & FS & 0.003 \\
\hline NBR & Wang-Brown Modified & FS & 0.003 \\
\hline UNF & Wang-Brown Modified & FS & 0.003 \\
\hline ESED & Bannantine-Socie & FS & 0.002 \\
\hline NBR & Bannantine-Socie & FS & 0.002 \\
\hline UNF & Bannantine-Socie & FS & 0.002 \\
\hline ESED & Wang-Brown & SWT & 0.000 \\
\hline NBR & Wang-Brown & SWT & 0.000 \\
\hline UNF & Wang-Brown & SWT & 0.000 \\
\hline ESED & Wang-Brown Modified & SWT & 0.000 \\
\hline NBR & Wang-Brown Modified & SWT & 0.000 \\
\hline UNF & Wang-Brown Modified & SWT & 0.000 \\
\hline ESED & Bannantine-Socie & SWT & 0.000 \\
\hline NBR & Bannantine-Socie & SWT & 0.000 \\
\hline UNF & Bannantine-Socie & SWT & 0.000 \\
\hline ESED & Wang-Brown & Liu VSE & 0.002 \\
\hline NBR & Wang-Brown & Liu VSE & 0.002 \\
\hline UNF & Wang-Brown & Liu VSE & 0.002 \\
\hline ESED & Wang-Brown Modified & Liu VSE & 0.004 \\
\hline NBR & Wang-Brown Modified & Liu VSE & 0.004 \\
\hline UNF & Wang-Brown Modified & Liu VSE & 0.004 \\
\hline ESED & Bannantine-Socie & Liu VSE & 0.002 \\
\hline NBR & Bannantine-Socie & Liu VSE & 0.002 \\
\hline UNF & Bannantine-Socie & Liu VSE & 0.002 \\
\hline
\end{tabular}


Table A.1-5: Fatigue damage results for Pin A at $90^{\circ}$ to the neutral axis and at the centre of the fuse groove $(\hat{z}=0)$.

\begin{tabular}{|c|c|c|c|}
\hline Notch Method & Cycle Count & Fatigue Criterion & Damage \\
\hline ESED & Wang-Brown & KBM & 0.001 \\
\hline NBR & Wang-Brown & KBM & 0.001 \\
\hline UNF & Wang-Brown & KBM & 0.001 \\
\hline ESED & Wang-Brown Modified & KBM & 0.001 \\
\hline NBR & Wang-Brown Modified & KBM & 0.001 \\
\hline UNF & Wang-Brown Modified & KBM & 0.001 \\
\hline ESED & Bannantine-Socie & KBM & 0.001 \\
\hline NBR & Bannantine-Socie & KBM & 0.001 \\
\hline UNF & Bannantine-Socie & KBM & 0.001 \\
\hline ESED & Wang-Brown & FS & 0.000 \\
\hline NBR & Wang-Brown & FS & 0.000 \\
\hline UNF & Wang-Brown & FS & 0.000 \\
\hline ESED & Wang-Brown Modified & FS & 0.000 \\
\hline NBR & Wang-Brown Modified & FS & 0.000 \\
\hline UNF & Wang-Brown Modified & FS & 0.000 \\
\hline ESED & Bannantine-Socie & FS & 0.000 \\
\hline NBR & Bannantine-Socie & FS & 0.000 \\
\hline UNF & Bannantine-Socie & FS & 0.000 \\
\hline ESED & Wang-Brown & SWT & 0.002 \\
\hline NBR & Wang-Brown & SWT & 0.002 \\
\hline UNF & Wang-Brown & SWT & 0.002 \\
\hline ESED & Wang-Brown Modified & SWT & 0.003 \\
\hline NBR & Wang-Brown Modified & SWT & 0.003 \\
\hline UNF & Wang-Brown Modified & SWT & 0.003 \\
\hline ESED & Bannantine-Socie & SWT & 0.002 \\
\hline NBR & Bannantine-Socie & SWT & 0.002 \\
\hline UNF & Bannantine-Socie & SWT & 0.002 \\
\hline ESED & Wang-Brown & Liu VSE & 0.000 \\
\hline NBR & Wang-Brown & Liu VSE & 0.000 \\
\hline UNF & Wang-Brown & Liu VSE & 0.000 \\
\hline ESED & Wang-Brown Modified & Liu VSE & 0.000 \\
\hline NBR & Wang-Brown Modified & Liu VSE & 0.000 \\
\hline UNF & Wang-Brown Modified & Liu VSE & 0.000 \\
\hline ESED & Bannantine-Socie & Liu VSE & 0.000 \\
\hline NBR & Bannantine-Socie & Liu VSE & 0.000 \\
\hline UNF & Bannantine-Socie & Liu VSE & 0.000 \\
\hline
\end{tabular}


Table A.1-6: Fatigue damage results for Pin A at $90^{\circ}$ to the neutral axis and offset $21 \%$ from the centre of the fuse groove $(\hat{z}=0.21)$.

\begin{tabular}{|c|c|c|c|}
\hline Notch Method & Cycle Count & Fatigue Criterion & Damage \\
\hline ESED & Wang-Brown & KBM & 0.079 \\
\hline NBR & Wang-Brown & KBM & 0.084 \\
\hline UNF & Wang-Brown & KBM & 0.078 \\
\hline ESED & Wang-Brown Modified & KBM & 0.151 \\
\hline NBR & Wang-Brown Modified & KBM & 0.160 \\
\hline UNF & Wang-Brown Modified & KBM & 0.150 \\
\hline ESED & Bannantine-Socie & KBM & 0.056 \\
\hline NBR & Bannantine-Socie & KBM & 0.060 \\
\hline UNF & Bannantine-Socie & KBM & 0.056 \\
\hline ESED & Wang-Brown & FS & 0.010 \\
\hline NBR & Wang-Brown & FS & 0.011 \\
\hline UNF & Wang-Brown & FS & 0.010 \\
\hline ESED & Wang-Brown Modified & FS & 0.020 \\
\hline NBR & Wang-Brown Modified & FS & 0.020 \\
\hline UNF & Wang-Brown Modified & FS & 0.020 \\
\hline ESED & Bannantine-Socie & FS & 0.011 \\
\hline NBR & Bannantine-Socie & FS & 0.011 \\
\hline UNF & Bannantine-Socie & FS & 0.011 \\
\hline ESED & Wang-Brown & SWT & 0.126 \\
\hline NBR & Wang-Brown & SWT & 0.142 \\
\hline UNF & Wang-Brown & SWT & 0.126 \\
\hline ESED & Wang-Brown Modified & SWT & 0.238 \\
\hline NBR & Wang-Brown Modified & SWT & 0.272 \\
\hline UNF & Wang-Brown Modified & SWT & 0.243 \\
\hline ESED & Bannantine-Socie & SWT & 0.133 \\
\hline NBR & Bannantine-Socie & SWT & 0.150 \\
\hline UNF & Bannantine-Socie & SWT & 0.134 \\
\hline ESED & Wang-Brown & Liu VSE & 0.011 \\
\hline NBR & Wang-Brown & Liu VSE & 0.011 \\
\hline UNF & Wang-Brown & Liu VSE & 0.011 \\
\hline ESED & Wang-Brown Modified & Liu VSE & 0.021 \\
\hline NBR & Wang-Brown Modified & Liu VSE & 0.021 \\
\hline UNF & Wang-Brown Modified & Liu VSE & 0.021 \\
\hline ESED & Bannantine-Socie & Liu VSE & 0.013 \\
\hline NBR & Bannantine-Socie & Liu VSE & 0.013 \\
\hline UNF & Bannantine-Socie & Liu VSE & 0.013 \\
\hline
\end{tabular}


Table A.1-7: Fatigue damage results for Pin A at $90^{\circ}$ to the neutral axis and offset $-21 \%$ from the centre of the fuse groove $(\hat{z}=-0.21)$.

\begin{tabular}{|c|c|c|c|}
\hline Notch Method & Cycle Count & Fatigue Criterion & Damage \\
\hline ESED & Wang-Brown & KBM & 0.000 \\
\hline NBR & Wang-Brown & KBM & 0.000 \\
\hline UNF & Wang-Brown & KBM & 0.000 \\
\hline ESED & Wang-Brown Modified & KBM & 0.001 \\
\hline NBR & Wang-Brown Modified & KBM & 0.001 \\
\hline UNF & Wang-Brown Modified & KBM & 0.001 \\
\hline ESED & Bannantine-Socie & KBM & 0.000 \\
\hline NBR & Bannantine-Socie & KBM & 0.000 \\
\hline UNF & Bannantine-Socie & KBM & 0.000 \\
\hline ESED & Wang-Brown & $\mathrm{FS}$ & 0.002 \\
\hline NBR & Wang-Brown & $\mathrm{FS}$ & 0.002 \\
\hline UNF & Wang-Brown & FS & 0.002 \\
\hline ESED & Wang-Brown Modified & FS & 0.003 \\
\hline NBR & Wang-Brown Modified & FS & 0.003 \\
\hline UNF & Wang-Brown Modified & FS & 0.003 \\
\hline ESED & Bannantine-Socie & FS & 0.002 \\
\hline NBR & Bannantine-Socie & FS & 0.002 \\
\hline UNF & Bannantine-Socie & FS & 0.002 \\
\hline ESED & Wang-Brown & SWT & 0.000 \\
\hline NBR & Wang-Brown & SWT & 0.000 \\
\hline UNF & Wang-Brown & SWT & 0.000 \\
\hline ESED & Wang-Brown Modified & SWT & 0.000 \\
\hline NBR & Wang-Brown Modified & SWT & 0.000 \\
\hline UNF & Wang-Brown Modified & SWT & 0.000 \\
\hline ESED & Bannantine-Socie & SWT & 0.000 \\
\hline NBR & Bannantine-Socie & SWT & 0.000 \\
\hline UNF & Bannantine-Socie & SWT & 0.000 \\
\hline ESED & Wang-Brown & Liu VSE & 0.003 \\
\hline NBR & Wang-Brown & Liu VSE & 0.003 \\
\hline UNF & Wang-Brown & Liu VSE & 0.003 \\
\hline ESED & Wang-Brown Modified & Liu VSE & 0.006 \\
\hline NBR & Wang-Brown Modified & Liu VSE & 0.006 \\
\hline UNF & Wang-Brown Modified & Liu VSE & 0.006 \\
\hline ESED & Bannantine-Socie & Liu VSE & 0.004 \\
\hline NBR & Bannantine-Socie & Liu VSE & 0.004 \\
\hline UNF & Bannantine-Socie & Liu VSE & 0.004 \\
\hline
\end{tabular}


Table A.1-8: Fatigue damage results for Pin A at $-45^{\circ}$ to the neutral axis and at the centre of the fuse groove $(\hat{z}=0)$.

\begin{tabular}{|c|c|c|c|}
\hline Notch Method & Cycle Count & Fatigue Criterion & Damage \\
\hline ESED & Wang-Brown & KBM & 0.061 \\
\hline NBR & Wang-Brown & KBM & 0.061 \\
\hline UNF & Wang-Brown & KBM & 0.061 \\
\hline ESED & Wang-Brown Modified & KBM & 0.116 \\
\hline NBR & Wang-Brown Modified & KBM & 0.116 \\
\hline UNF & Wang-Brown Modified & KBM & 0.116 \\
\hline ESED & Bannantine-Socie & KBM & 0.071 \\
\hline NBR & Bannantine-Socie & KBM & 0.071 \\
\hline UNF & Bannantine-Socie & KBM & 0.071 \\
\hline ESED & Wang-Brown & FS & 0.009 \\
\hline NBR & Wang-Brown & FS & 0.009 \\
\hline UNF & Wang-Brown & FS & 0.009 \\
\hline ESED & Wang-Brown Modified & FS & 0.017 \\
\hline NBR & Wang-Brown Modified & FS & 0.017 \\
\hline UNF & Wang-Brown Modified & FS & 0.017 \\
\hline ESED & Bannantine-Socie & FS & 0.010 \\
\hline NBR & Bannantine-Socie & FS & 0.010 \\
\hline UNF & Bannantine-Socie & FS & 0.010 \\
\hline ESED & Wang-Brown & SWT & 0.010 \\
\hline NBR & Wang-Brown & SWT & 0.010 \\
\hline UNF & Wang-Brown & SWT & 0.010 \\
\hline ESED & Wang-Brown Modified & SWT & 0.018 \\
\hline NBR & Wang-Brown Modified & SWT & 0.018 \\
\hline UNF & Wang-Brown Modified & SWT & 0.018 \\
\hline ESED & Bannantine-Socie & SWT & 0.010 \\
\hline NBR & Bannantine-Socie & SWT & 0.010 \\
\hline UNF & Bannantine-Socie & SWT & 0.010 \\
\hline ESED & Wang-Brown & Liu VSE & 0.008 \\
\hline NBR & Wang-Brown & Liu VSE & 0.008 \\
\hline UNF & Wang-Brown & Liu VSE & 0.008 \\
\hline ESED & Wang-Brown Modified & Liu VSE & 0.016 \\
\hline NBR & Wang-Brown Modified & Liu VSE & 0.016 \\
\hline UNF & Wang-Brown Modified & Liu VSE & 0.016 \\
\hline ESED & Bannantine-Socie & Liu VSE & 0.010 \\
\hline NBR & Bannantine-Socie & Liu VSE & 0.010 \\
\hline UNF & Bannantine-Socie & Liu VSE & 0.010 \\
\hline
\end{tabular}


Table A.1-9: Fatigue damage results for Pin A at $-45^{\circ}$ to the neutral axis and offset $19 \%$ from the centre of the fuse groove $(\hat{z}=0.19)$.

\begin{tabular}{|c|c|c|c|}
\hline Notch Method & Cycle Count & Fatigue Criterion & Damage \\
\hline ESED & Wang-Brown & KBM & 0.003 \\
\hline NBR & Wang-Brown & KBM & 0.003 \\
\hline UNF & Wang-Brown & KBM & 0.003 \\
\hline ESED & Wang-Brown Modified & KBM & 0.006 \\
\hline NBR & Wang-Brown Modified & KBM & 0.005 \\
\hline UNF & Wang-Brown Modified & KBM & 0.006 \\
\hline ESED & Bannantine-Socie & KBM & 0.004 \\
\hline NBR & Bannantine-Socie & KBM & 0.004 \\
\hline UNF & Bannantine-Socie & KBM & 0.004 \\
\hline ESED & Wang-Brown & FS & 0.015 \\
\hline NBR & Wang-Brown & FS & 0.015 \\
\hline UNF & Wang-Brown & FS & 0.015 \\
\hline ESED & Wang-Brown Modified & FS & 0.029 \\
\hline NBR & Wang-Brown Modified & FS & 0.029 \\
\hline UNF & Wang-Brown Modified & FS & 0.029 \\
\hline ESED & Bannantine-Socie & FS & 0.019 \\
\hline NBR & Bannantine-Socie & FS & 0.019 \\
\hline UNF & Bannantine-Socie & FS & 0.019 \\
\hline ESED & Wang-Brown & SWT & 0.000 \\
\hline NBR & Wang-Brown & SWT & 0.000 \\
\hline UNF & Wang-Brown & SWT & 0.000 \\
\hline ESED & Wang-Brown Modified & SWT & 0.000 \\
\hline NBR & Wang-Brown Modified & SWT & 0.000 \\
\hline UNF & Wang-Brown Modified & SWT & 0.000 \\
\hline ESED & Bannantine-Socie & SWT & 0.000 \\
\hline NBR & Bannantine-Socie & SWT & 0.000 \\
\hline UNF & Bannantine-Socie & SWT & 0.000 \\
\hline ESED & Wang-Brown & Liu VSE & 0.034 \\
\hline NBR & Wang-Brown & Liu VSE & 0.034 \\
\hline UNF & Wang-Brown & Liu VSE & 0.034 \\
\hline ESED & Wang-Brown Modified & Liu VSE & 0.066 \\
\hline NBR & Wang-Brown Modified & Liu VSE & 0.067 \\
\hline UNF & Wang-Brown Modified & Liu VSE & 0.066 \\
\hline ESED & Bannantine-Socie & Liu VSE & 0.044 \\
\hline NBR & Bannantine-Socie & Liu VSE & 0.045 \\
\hline UNF & Bannantine-Socie & Liu VSE & 0.044 \\
\hline
\end{tabular}


Table A.1-10: Fatigue damage results for Pin A at $-45^{\circ}$ to the neutral axis and offset $-19 \%$ from the centre of the fuse groove $(\hat{z}=-0.19)$.

\begin{tabular}{|c|c|c|c|}
\hline Notch Method & Cycle Count & Fatigue Criterion & Damage \\
\hline ESED & Wang-Brown & KBM & 0.360 \\
\hline NBR & Wang-Brown & KBM & 0.360 \\
\hline UNF & Wang-Brown & KBM & 0.360 \\
\hline ESED & Wang-Brown Modified & KBM & 0.695 \\
\hline NBR & Wang-Brown Modified & KBM & 0.695 \\
\hline UNF & Wang-Brown Modified & KBM & 0.695 \\
\hline ESED & Bannantine-Socie & KBM & 0.414 \\
\hline NBR & Bannantine-Socie & KBM & 0.414 \\
\hline UNF & Bannantine-Socie & KBM & 0.414 \\
\hline ESED & Wang-Brown & $\mathrm{FS}$ & 0.002 \\
\hline NBR & Wang-Brown & $\mathrm{FS}$ & 0.002 \\
\hline UNF & Wang-Brown & FS & 0.002 \\
\hline ESED & Wang-Brown Modified & FS & 0.004 \\
\hline NBR & Wang-Brown Modified & FS & 0.004 \\
\hline UNF & Wang-Brown Modified & FS & 0.004 \\
\hline ESED & Bannantine-Socie & FS & 0.003 \\
\hline NBR & Bannantine-Socie & FS & 0.003 \\
\hline UNF & Bannantine-Socie & FS & 0.003 \\
\hline ESED & Wang-Brown & SWT & 0.002 \\
\hline NBR & Wang-Brown & SWT & 0.002 \\
\hline UNF & Wang-Brown & SWT & 0.002 \\
\hline ESED & Wang-Brown Modified & SWT & 0.004 \\
\hline NBR & Wang-Brown Modified & SWT & 0.004 \\
\hline UNF & Wang-Brown Modified & SWT & 0.004 \\
\hline ESED & Bannantine-Socie & SWT & 0.006 \\
\hline NBR & Bannantine-Socie & SWT & 0.006 \\
\hline UNF & Bannantine-Socie & SWT & 0.006 \\
\hline ESED & Wang-Brown & Liu VSE & 0.001 \\
\hline NBR & Wang-Brown & Liu VSE & 0.001 \\
\hline UNF & Wang-Brown & Liu VSE & 0.001 \\
\hline ESED & Wang-Brown Modified & Liu VSE & 0.001 \\
\hline NBR & Wang-Brown Modified & Liu VSE & 0.001 \\
\hline UNF & Wang-Brown Modified & Liu VSE & 0.001 \\
\hline ESED & Bannantine-Socie & Liu VSE & 0.001 \\
\hline NBR & Bannantine-Socie & Liu VSE & 0.001 \\
\hline UNF & Bannantine-Socie & Liu VSE & 0.001 \\
\hline
\end{tabular}


Table A.1-11: Fatigue damage results for Pin A at $-90^{\circ}$ to the neutral axis and the centre of the fuse groove $(\hat{z}=0)$.

\begin{tabular}{|c|c|c|c|}
\hline Notch Method & Cycle Count & Fatigue Criterion & Damage \\
\hline ESED & Wang-Brown & KBM & 0.001 \\
\hline NBR & Wang-Brown & KBM & 0.001 \\
\hline UNF & Wang-Brown & KBM & 0.001 \\
\hline ESED & Wang-Brown Modified & KBM & 0.002 \\
\hline NBR & Wang-Brown Modified & KBM & 0.002 \\
\hline UNF & Wang-Brown Modified & KBM & 0.002 \\
\hline ESED & Bannantine-Socie & KBM & 0.002 \\
\hline NBR & Bannantine-Socie & KBM & 0.002 \\
\hline UNF & Bannantine-Socie & KBM & 0.002 \\
\hline ESED & Wang-Brown & $\mathrm{FS}$ & 0.000 \\
\hline NBR & Wang-Brown & $\mathrm{FS}$ & 0.000 \\
\hline UNF & Wang-Brown & FS & 0.000 \\
\hline ESED & Wang-Brown Modified & FS & 0.001 \\
\hline NBR & Wang-Brown Modified & FS & 0.001 \\
\hline UNF & Wang-Brown Modified & FS & 0.001 \\
\hline ESED & Bannantine-Socie & FS & 0.000 \\
\hline NBR & Bannantine-Socie & FS & 0.000 \\
\hline UNF & Bannantine-Socie & FS & 0.000 \\
\hline ESED & Wang-Brown & SWT & 0.003 \\
\hline NBR & Wang-Brown & SWT & 0.003 \\
\hline UNF & Wang-Brown & SWT & 0.003 \\
\hline ESED & Wang-Brown Modified & SWT & 0.006 \\
\hline NBR & Wang-Brown Modified & SWT & 0.006 \\
\hline UNF & Wang-Brown Modified & SWT & 0.006 \\
\hline ESED & Bannantine-Socie & SWT & 0.003 \\
\hline NBR & Bannantine-Socie & SWT & 0.003 \\
\hline UNF & Bannantine-Socie & SWT & 0.003 \\
\hline ESED & Wang-Brown & Liu VSE & 0.000 \\
\hline NBR & Wang-Brown & Liu VSE & 0.000 \\
\hline UNF & Wang-Brown & Liu VSE & 0.000 \\
\hline ESED & Wang-Brown Modified & Liu VSE & 0.001 \\
\hline NBR & Wang-Brown Modified & Liu VSE & 0.001 \\
\hline UNF & Wang-Brown Modified & Liu VSE & 0.001 \\
\hline ESED & Bannantine-Socie & Liu VSE & 0.000 \\
\hline NBR & Bannantine-Socie & Liu VSE & 0.000 \\
\hline UNF & Bannantine-Socie & Liu VSE & 0.000 \\
\hline
\end{tabular}


Table A.1-12: Fatigue damage results for Pin A at $-90^{\circ}$ to the neutral axis and offset $21 \%$ from the centre of the fuse groove $(\hat{z}=21 \%)$.

\begin{tabular}{|c|c|c|c|}
\hline Notch Method & Cycle Count & Fatigue Criterion & Damage \\
\hline ESED & Wang-Brown & KBM & 0.001 \\
\hline NBR & Wang-Brown & KBM & 0.001 \\
\hline UNF & Wang-Brown & KBM & 0.001 \\
\hline ESED & Wang-Brown Modified & KBM & 0.002 \\
\hline NBR & Wang-Brown Modified & KBM & 0.002 \\
\hline UNF & Wang-Brown Modified & KBM & 0.002 \\
\hline ESED & Bannantine-Socie & KBM & 0.002 \\
\hline NBR & Bannantine-Socie & KBM & 0.002 \\
\hline UNF & Bannantine-Socie & KBM & 0.002 \\
\hline ESED & Wang-Brown & FS & 0.000 \\
\hline NBR & Wang-Brown & FS & 0.000 \\
\hline UNF & Wang-Brown & FS & 0.000 \\
\hline ESED & Wang-Brown Modified & FS & 0.001 \\
\hline NBR & Wang-Brown Modified & FS & 0.001 \\
\hline UNF & Wang-Brown Modified & FS & 0.001 \\
\hline ESED & Bannantine-Socie & FS & 0.000 \\
\hline NBR & Bannantine-Socie & FS & 0.000 \\
\hline UNF & Bannantine-Socie & FS & 0.000 \\
\hline ESED & Wang-Brown & SWT & 0.003 \\
\hline NBR & Wang-Brown & SWT & 0.003 \\
\hline UNF & Wang-Brown & SWT & 0.003 \\
\hline ESED & Wang-Brown Modified & SWT & 0.006 \\
\hline NBR & Wang-Brown Modified & SWT & 0.006 \\
\hline UNF & Wang-Brown Modified & SWT & 0.006 \\
\hline ESED & Bannantine-Socie & SWT & 0.003 \\
\hline NBR & Bannantine-Socie & SWT & 0.003 \\
\hline UNF & Bannantine-Socie & SWT & 0.003 \\
\hline ESED & Wang-Brown & Liu VSE & 0.000 \\
\hline NBR & Wang-Brown & Liu VSE & 0.000 \\
\hline UNF & Wang-Brown & Liu VSE & 0.000 \\
\hline ESED & Wang-Brown Modified & Liu VSE & 0.001 \\
\hline NBR & Wang-Brown Modified & Liu VSE & 0.001 \\
\hline UNF & Wang-Brown Modified & Liu VSE & 0.001 \\
\hline ESED & Bannantine-Socie & Liu VSE & 0.000 \\
\hline NBR & Bannantine-Socie & Liu VSE & 0.000 \\
\hline UNF & Bannantine-Socie & Liu VSE & 0.000 \\
\hline
\end{tabular}


Table A.1-13: Fatigue damage results for Pin A at $-90^{\circ}$ to the neutral axis and offset $-21 \%$ from the centre of the fuse groove $(\hat{z}=-21 \%)$.

\begin{tabular}{|c|c|c|c|}
\hline Notch Method & Cycle Count & Fatigue Criterion & Damage \\
\hline ESED & Wang-Brown & KBM & 0.002 \\
\hline NBR & Wang-Brown & KBM & 0.002 \\
\hline UNF & Wang-Brown & KBM & 0.002 \\
\hline ESED & Wang-Brown Modified & KBM & 0.005 \\
\hline NBR & Wang-Brown Modified & KBM & 0.004 \\
\hline UNF & Wang-Brown Modified & KBM & 0.005 \\
\hline ESED & Bannantine-Socie & KBM & 0.003 \\
\hline NBR & Bannantine-Socie & KBM & 0.003 \\
\hline UNF & Bannantine-Socie & KBM & 0.003 \\
\hline ESED & Wang-Brown & FS & 0.013 \\
\hline NBR & Wang-Brown & FS & 0.013 \\
\hline UNF & Wang-Brown & FS & 0.013 \\
\hline ESED & Wang-Brown Modified & FS & 0.025 \\
\hline NBR & Wang-Brown Modified & FS & 0.025 \\
\hline UNF & Wang-Brown Modified & FS & 0.025 \\
\hline ESED & Bannantine-Socie & FS & 0.016 \\
\hline NBR & Bannantine-Socie & FS & 0.016 \\
\hline UNF & Bannantine-Socie & FS & 0.016 \\
\hline ESED & Wang-Brown & SWT & 0.000 \\
\hline NBR & Wang-Brown & SWT & 0.000 \\
\hline UNF & Wang-Brown & SWT & 0.000 \\
\hline ESED & Wang-Brown Modified & SWT & 0.000 \\
\hline NBR & Wang-Brown Modified & SWT & 0.000 \\
\hline UNF & Wang-Brown Modified & SWT & 0.000 \\
\hline ESED & Bannantine-Socie & SWT & 0.000 \\
\hline NBR & Bannantine-Socie & SWT & 0.000 \\
\hline UNF & Bannantine-Socie & SWT & 0.000 \\
\hline ESED & Wang-Brown & Liu VSE & 0.031 \\
\hline NBR & Wang-Brown & Liu VSE & 0.031 \\
\hline UNF & Wang-Brown & Liu VSE & 0.031 \\
\hline ESED & Wang-Brown Modified & Liu VSE & 0.061 \\
\hline NBR & Wang-Brown Modified & Liu VSE & 0.061 \\
\hline UNF & Wang-Brown Modified & Liu VSE & 0.061 \\
\hline ESED & Bannantine-Socie & Liu VSE & 0.039 \\
\hline NBR & Bannantine-Socie & Liu VSE & 0.040 \\
\hline UNF & Bannantine-Socie & Liu VSE & 0.040 \\
\hline
\end{tabular}




\section{A.2 Pin A Test-Based Fatigue Damage Results}

Table A.2-1: Fatigue damage results for Pin A at the neutral axis and centre of the fuse groove $(\hat{z}=0)$. Input stresses calculated using strain test data.

\begin{tabular}{|c|c|c|c|}
\hline Notch Method & Cycle Count & Fatigue Criterion & Damage \\
\hline ESED & Wang-Brown & KBM & 1.020 \\
\hline NBR & Wang-Brown & KBM & 0.791 \\
\hline UNF & Wang-Brown & KBM & 0.521 \\
\hline ESED & Wang-Brown Modified & KBM & 1.982 \\
\hline NBR & Wang-Brown Modified & KBM & 1.558 \\
\hline UNF & Wang-Brown Modified & KBM & 0.998 \\
\hline ESED & Bannantine-Socie & KBM & 1.113 \\
\hline NBR & Bannantine-Socie & KBM & 1.307 \\
\hline UNF & Bannantine-Socie & KBM & 1.002 \\
\hline ESED & Wang-Brown & FS & 0.807 \\
\hline NBR & Wang-Brown & FS & 0.781 \\
\hline UNF & Wang-Brown & FS & 0.680 \\
\hline ESED & Wang-Brown Modified & FS & 1.567 \\
\hline NBR & Wang-Brown Modified & FS & 1.540 \\
\hline UNF & Wang-Brown Modified & FS & 1.309 \\
\hline ESED & Bannantine-Socie & FS & 0.882 \\
\hline NBR & Bannantine-Socie & FS & 0.885 \\
\hline UNF & Bannantine-Socie & FS & 0.824 \\
\hline ESED & Wang-Brown & SWT & 0.050 \\
\hline NBR & Wang-Brown & SWT & 0.084 \\
\hline UNF & Wang-Brown & SWT & 0.035 \\
\hline ESED & Wang-Brown Modified & SWT & 0.092 \\
\hline NBR & Wang-Brown Modified & SWT & 0.162 \\
\hline UNF & Wang-Brown Modified & SWT & 0.067 \\
\hline ESED & Bannantine-Socie & SWT & 0.063 \\
\hline NBR & Bannantine-Socie & SWT & 0.111 \\
\hline UNF & Bannantine-Socie & SWT & 0.050 \\
\hline ESED & Wang-Brown & Liu VSE & 1.917 \\
\hline NBR & Wang-Brown & Liu VSE & 2.020 \\
\hline UNF & Wang-Brown & Liu VSE & 1.941 \\
\hline ESED & Wang-Brown Modified & Liu VSE & 3.703 \\
\hline NBR & Wang-Brown Modified & Liu VSE & 3.978 \\
\hline UNF & Wang-Brown Modified & Liu VSE & 3.733 \\
\hline ESED & Bannantine-Socie & Liu VSE & 2.207 \\
\hline NBR & Bannantine-Socie & Liu VSE & 2.334 \\
\hline UNF & Bannantine-Socie & Liu VSE & 2.231 \\
\hline
\end{tabular}


Table A.2-2: Fatigue damage results for Pin A at $45^{\circ}$ to the neutral axis and centre of the fuse groove $(\hat{z}=0)$.

\begin{tabular}{|c|c|c|c|}
\hline Notch Method & Cycle Count & Fatigue Criterion & Damage \\
\hline ESED & Wang-Brown & KBM & 0.011 \\
\hline NBR & Wang-Brown & KBM & 0.011 \\
\hline UNF & Wang-Brown & KBM & 0.011 \\
\hline ESED & Wang-Brown Modified & KBM & 0.020 \\
\hline NBR & Wang-Brown Modified & KBM & 0.020 \\
\hline UNF & Wang-Brown Modified & KBM & 0.020 \\
\hline ESED & Bannantine-Socie & KBM & 0.013 \\
\hline NBR & Bannantine-Socie & KBM & 0.013 \\
\hline UNF & Bannantine-Socie & KBM & 0.013 \\
\hline ESED & Wang-Brown & $\mathrm{FS}$ & 0.003 \\
\hline NBR & Wang-Brown & $\mathrm{FS}$ & 0.003 \\
\hline UNF & Wang-Brown & FS & 0.003 \\
\hline ESED & Wang-Brown Modified & FS & 0.006 \\
\hline NBR & Wang-Brown Modified & FS & 0.006 \\
\hline UNF & Wang-Brown Modified & FS & 0.006 \\
\hline ESED & Bannantine-Socie & FS & 0.004 \\
\hline NBR & Bannantine-Socie & FS & 0.004 \\
\hline UNF & Bannantine-Socie & FS & 0.004 \\
\hline ESED & Wang-Brown & SWT & 0.002 \\
\hline NBR & Wang-Brown & SWT & 0.002 \\
\hline UNF & Wang-Brown & SWT & 0.002 \\
\hline ESED & Wang-Brown Modified & SWT & 0.003 \\
\hline NBR & Wang-Brown Modified & SWT & 0.003 \\
\hline UNF & Wang-Brown Modified & SWT & 0.003 \\
\hline ESED & Bannantine-Socie & SWT & 0.002 \\
\hline NBR & Bannantine-Socie & SWT & 0.002 \\
\hline UNF & Bannantine-Socie & SWT & 0.002 \\
\hline ESED & Wang-Brown & Liu VSE & 0.003 \\
\hline NBR & Wang-Brown & Liu VSE & 0.003 \\
\hline UNF & Wang-Brown & Liu VSE & 0.003 \\
\hline ESED & Wang-Brown Modified & Liu VSE & 0.006 \\
\hline NBR & Wang-Brown Modified & Liu VSE & 0.006 \\
\hline UNF & Wang-Brown Modified & Liu VSE & 0.006 \\
\hline ESED & Bannantine-Socie & Liu VSE & 0.004 \\
\hline NBR & Bannantine-Socie & Liu VSE & 0.004 \\
\hline UNF & Bannantine-Socie & Liu VSE & 0.004 \\
\hline
\end{tabular}


Table A.2-3: Fatigue damage results for Pin A at $90^{\circ}$ to the neutral axis and at the centre of the fuse groove $(\hat{z}=0)$.

\begin{tabular}{|c|c|c|c|}
\hline Notch Method & Cycle Count & Fatigue Criterion & Damage \\
\hline ESED & Wang-Brown & KBM & 0.001 \\
\hline NBR & Wang-Brown & KBM & 0.001 \\
\hline UNF & Wang-Brown & KBM & 0.001 \\
\hline ESED & Wang-Brown Modified & KBM & 0.001 \\
\hline NBR & Wang-Brown Modified & KBM & 0.001 \\
\hline UNF & Wang-Brown Modified & KBM & 0.001 \\
\hline ESED & Bannantine-Socie & KBM & 0.001 \\
\hline NBR & Bannantine-Socie & KBM & 0.001 \\
\hline UNF & Bannantine-Socie & KBM & 0.001 \\
\hline ESED & Wang-Brown & FS & 0.000 \\
\hline NBR & Wang-Brown & FS & 0.000 \\
\hline UNF & Wang-Brown & FS & 0.000 \\
\hline ESED & Wang-Brown Modified & FS & 0.000 \\
\hline NBR & Wang-Brown Modified & FS & 0.000 \\
\hline UNF & Wang-Brown Modified & FS & 0.000 \\
\hline ESED & Bannantine-Socie & FS & 0.000 \\
\hline NBR & Bannantine-Socie & FS & 0.000 \\
\hline UNF & Bannantine-Socie & FS & 0.000 \\
\hline ESED & Wang-Brown & SWT & 0.002 \\
\hline NBR & Wang-Brown & SWT & 0.002 \\
\hline UNF & Wang-Brown & SWT & 0.002 \\
\hline ESED & Wang-Brown Modified & SWT & 0.003 \\
\hline NBR & Wang-Brown Modified & SWT & 0.003 \\
\hline UNF & Wang-Brown Modified & SWT & 0.003 \\
\hline ESED & Bannantine-Socie & SWT & 0.002 \\
\hline NBR & Bannantine-Socie & SWT & 0.002 \\
\hline UNF & Bannantine-Socie & SWT & 0.002 \\
\hline ESED & Wang-Brown & Liu VSE & 0.000 \\
\hline NBR & Wang-Brown & Liu VSE & 0.000 \\
\hline UNF & Wang-Brown & Liu VSE & 0.000 \\
\hline ESED & Wang-Brown Modified & Liu VSE & 0.000 \\
\hline NBR & Wang-Brown Modified & Liu VSE & 0.000 \\
\hline UNF & Wang-Brown Modified & Liu VSE & 0.000 \\
\hline ESED & Bannantine-Socie & Liu VSE & 0.000 \\
\hline NBR & Bannantine-Socie & Liu VSE & 0.000 \\
\hline UNF & Bannantine-Socie & Liu VSE & 0.000 \\
\hline
\end{tabular}




\section{A.3 Pin B FEA-Based Fatigue Damage Results}

Table A.3-1: Fatigue damage results for Pin B at the neutral axis and centre of the fuse groove ( $\hat{z}$ $=0)$. Input stresses determined from elastic FEA data.

\begin{tabular}{|c|c|c|c|}
\hline Notch Method & Cycle Count & Fatigue Criterion & Damage \\
\hline ESED & Wang-Brown & KBM & 0.088 \\
\hline NBR & Wang-Brown & KBM & 0.092 \\
\hline UNF & Wang-Brown & KBM & 0.089 \\
\hline ESED & Wang-Brown Modified & KBM & 0.162 \\
\hline NBR & Wang-Brown Modified & KBM & 0.170 \\
\hline UNF & Wang-Brown Modified & KBM & 0.164 \\
\hline ESED & Bannantine-Socie & KBM & 0.080 \\
\hline NBR & Bannantine-Socie & KBM & 0.082 \\
\hline UNF & Bannantine-Socie & KBM & 0.081 \\
\hline ESED & Wang-Brown & FS & 0.144 \\
\hline NBR & Wang-Brown & FS & 0.149 \\
\hline UNF & Wang-Brown & FS & 0.145 \\
\hline ESED & Wang-Brown Modified & FS & 0.266 \\
\hline NBR & Wang-Brown Modified & FS & 0.275 \\
\hline UNF & Wang-Brown Modified & FS & 0.266 \\
\hline ESED & Bannantine-Socie & FS & 0.133 \\
\hline NBR & Bannantine-Socie & FS & 0.134 \\
\hline UNF & Bannantine-Socie & FS & 0.133 \\
\hline ESED & Wang-Brown & SWT & 0.000 \\
\hline NBR & Wang-Brown & SWT & 0.000 \\
\hline UNF & Wang-Brown & SWT & 0.000 \\
\hline ESED & Wang-Brown Modified & SWT & 0.000 \\
\hline NBR & Wang-Brown Modified & SWT & 0.000 \\
\hline UNF & Wang-Brown Modified & SWT & 0.000 \\
\hline ESED & Bannantine-Socie & SWT & 0.000 \\
\hline NBR & Bannantine-Socie & SWT & 0.000 \\
\hline UNF & Bannantine-Socie & SWT & 0.000 \\
\hline ESED & Wang-Brown & Liu VSE & 0.200 \\
\hline NBR & Wang-Brown & Liu VSE & 0.204 \\
\hline UNF & Wang-Brown & Liu VSE & 0.200 \\
\hline ESED & Wang-Brown Modified & Liu VSE & 0.368 \\
\hline NBR & Wang-Brown Modified & Liu VSE & 0.377 \\
\hline UNF & Wang-Brown Modified & Liu VSE & 0.368 \\
\hline ESED & Bannantine-Socie & Liu VSE & 0.187 \\
\hline NBR & Bannantine-Socie & Liu VSE & 0.188 \\
\hline
\end{tabular}




\begin{tabular}{|l|l|l|r|}
\hline Notch Method & Cycle Count & Fatigue Criterion & Damage \\
\hline UNF & Bannantine-Socie & Liu VSE & 0.187 \\
\hline
\end{tabular}

Table A.3-2: Fatigue damage results for Pin B at $45^{\circ}$ to the neutral axis and at the centre of the fuse groove $(\hat{z}=0)$. Input stresses determined from elastic FEA data.

\begin{tabular}{|l|l|l|r|}
\hline Notch Method & Cycle Count & Fatigue Criterion & Damage \\
\hline ESED & Wang-Brown & KBM & 0.000 \\
\hline NBR & Wang-Brown & KBM & 0.000 \\
\hline UNF & Wang-Brown & KBM & 0.000 \\
\hline ESED & Wang-Brown Modified & KBM & 0.000 \\
\hline NBR & Wang-Brown Modified & KBM & 0.000 \\
\hline UNF & Wang-Brown Modified & KBM & 0.000 \\
\hline ESED & Bannantine-Socie & KBM & 0.000 \\
\hline NBR & Bannantine-Socie & KBM & 0.000 \\
\hline UNF & Bannantine-Socie & KBM & 0.000 \\
\hline ESED & Wang-Brown & FS & 0.000 \\
\hline NBR & Wang-Brown & FS & 0.000 \\
\hline UNF & Wang-Brown & FS & 0.000 \\
\hline ESED & Wang-Brown Modified & FS & 0.000 \\
\hline NBR & Wang-Brown Modified & FS & 0.000 \\
\hline UNF & Wang-Brown Modified & FS & 0.000 \\
\hline ESED & Bannantine-Socie & FS & 0.000 \\
\hline NBR & Bannantine-Socie & FS & 0.000 \\
\hline UNF & Bannantine-Socie & FS & 0.000 \\
\hline ESED & Wang-Brown & SWT & 0.000 \\
\hline NBR & Wang-Brown & SWT & 0.000 \\
\hline UNF & Wang-Brown & SWT & 0.000 \\
\hline ESED & Wang-Brown Modified & SWT & 0.000 \\
\hline NBR & Wang-Brown Modified & SWT & 0.000 \\
\hline UNF & Wang-Brown Modified & SWT & 0.000 \\
\hline ESED & Bannantine-Socie & SWT & 0.000 \\
\hline NBR & Bannantine-Socie & SWT & 0.000 \\
\hline UNF & Bannantine-Socie & SWT & 0.000 \\
\hline ESED & Wang-Brown & Liu VSE & 0.000 \\
\hline NBR & Wang-Brown & Liu VSE & 0.000 \\
\hline UNF & Wang-Brown & Liu VSE & 0.000 \\
\hline ESED & Wang-Brown Modified & Liu VSE & 0.000 \\
\hline NBR & Wang-Brown Modified & Liu VSE & 0.000 \\
\hline UNF & Wang-Brown Modified & Liu VSE & 0.000 \\
\hline ESED & Bannantine-Socie & Liu VSE & 0.000 \\
\hline & & & \\
\hline
\end{tabular}




\begin{tabular}{|l|l|l|r|}
\hline Notch Method & Cycle Count & Fatigue Criterion & Damage \\
\hline NBR & Bannantine-Socie & Liu VSE & 0.000 \\
\hline UNF & Bannantine-Socie & Liu VSE & 0.000 \\
\hline
\end{tabular}

Table A.3-3: Fatigue damage results for Pin B at $45^{\circ}$ to the neutral axis and offset $35 \%$ from the centre of the fuse groove $(\hat{\mathrm{z}}=0.35)$. Input stresses determined from elastic FEA data.

\begin{tabular}{|l|l|l|r|}
\hline Notch Method & Cycle Count & Fatigue Criterion & Damage \\
\hline ESED & Wang-Brown & KBM & 0.005 \\
\hline NBR & Wang-Brown & KBM & 0.005 \\
\hline UNF & Wang-Brown & KBM & 0.005 \\
\hline ESED & Wang-Brown Modified & KBM & 0.010 \\
\hline NBR & Wang-Brown Modified & KBM & 0.010 \\
\hline UNF & Wang-Brown Modified & KBM & 0.010 \\
\hline ESED & Bannantine-Socie & KBM & 0.005 \\
\hline NBR & Bannantine-Socie & KBM & 0.005 \\
\hline UNF & Bannantine-Socie & KBM & 0.005 \\
\hline ESED & Wang-Brown & FS & 0.001 \\
\hline NBR & Wang-Brown & FS & 0.001 \\
\hline UNF & Wang-Brown & FS & 0.001 \\
\hline ESED & Wang-Brown Modified & FS & 0.003 \\
\hline NBR & Wang-Brown Modified & FS & 0.003 \\
\hline UNF & Wang-Brown Modified & FS & 0.003 \\
\hline ESED & Bannantine-Socie & FS & 0.001 \\
\hline NBR & Bannantine-Socie & FS & 0.001 \\
\hline UNF & Bannantine-Socie & FS & 0.001 \\
\hline ESED & Wang-Brown & SWT & 0.006 \\
\hline NBR & Wang-Brown & SWT & 0.006 \\
\hline UNF & Wang-Brown & SWT & 0.006 \\
\hline ESED & Wang-Brown Modified & SWT & 0.011 \\
\hline NBR & Wang-Brown Modified & SWT & 0.011 \\
\hline UNF & Wang-Brown Modified & SWT & 0.011 \\
\hline ESED & Bannantine-Socie & SWT & 0.006 \\
\hline NBR & Bannantine-Socie & SWT & 0.006 \\
\hline UNF & Wang-Brown & SWT & 0.001 \\
\hline ESED & Wang-Brown & Liu VSE & 0.001 \\
\hline NBR & Liu VSE & 0.001 \\
\hline UNF & Liu VSE & 0.002 \\
\hline ESED & & \\
\hline & & Sang-Brown Modified & SWE \\
\hline
\end{tabular}




\begin{tabular}{|l|l|l|r|}
\hline Notch Method & Cycle Count & Fatigue Criterion & Damage \\
\hline NBR & Wang-Brown Modified & Liu VSE & 0.002 \\
\hline UNF & Wang-Brown Modified & Liu VSE & 0.002 \\
\hline ESED & Bannantine-Socie & Liu VSE & 0.001 \\
\hline NBR & Bannantine-Socie & Liu VSE & 0.001 \\
\hline UNF & Bannantine-Socie & Liu VSE & 0.001 \\
\hline
\end{tabular}

Table A.3-4: Fatigue damage results for Pin B at $45^{\circ}$ to the neutral axis and offset $21 \%$ from the centre of the fuse groove $(\hat{\mathrm{z}}=-0.21)$. Input stresses determined from elastic FEA data.

\begin{tabular}{|c|c|c|c|}
\hline Notch Method & Cycle Count & Fatigue Criterion & Damage \\
\hline ESED & Wang-Brown & KBM & 0.000 \\
\hline NBR & Wang-Brown & KBM & 0.000 \\
\hline UNF & Wang-Brown & KBM & 0.000 \\
\hline ESED & Wang-Brown Modified & KBM & 0.000 \\
\hline NBR & Wang-Brown Modified & KBM & 0.000 \\
\hline UNF & Wang-Brown Modified & KBM & 0.000 \\
\hline ESED & Bannantine-Socie & KBM & 0.000 \\
\hline NBR & Bannantine-Socie & KBM & 0.000 \\
\hline UNF & Bannantine-Socie & KBM & 0.000 \\
\hline ESED & Wang-Brown & $\mathrm{FS}$ & 0.000 \\
\hline NBR & Wang-Brown & FS & 0.000 \\
\hline UNF & Wang-Brown & FS & 0.000 \\
\hline ESED & Wang-Brown Modified & FS & 0.000 \\
\hline NBR & Wang-Brown Modified & $\mathrm{FS}$ & 0.000 \\
\hline UNF & Wang-Brown Modified & FS & 0.000 \\
\hline ESED & Bannantine-Socie & FS & 0.000 \\
\hline NBR & Bannantine-Socie & FS & 0.000 \\
\hline UNF & Bannantine-Socie & FS & 0.000 \\
\hline ESED & Wang-Brown & SWT & 0.000 \\
\hline NBR & Wang-Brown & SWT & 0.000 \\
\hline UNF & Wang-Brown & SWT & 0.000 \\
\hline ESED & Wang-Brown Modified & SWT & 0.000 \\
\hline NBR & Wang-Brown Modified & SWT & 0.000 \\
\hline UNF & Wang-Brown Modified & SWT & 0.000 \\
\hline ESED & Bannantine-Socie & SWT & 0.000 \\
\hline NBR & Bannantine-Socie & SWT & 0.000 \\
\hline UNF & Bannantine-Socie & SWT & 0.000 \\
\hline ESED & Wang-Brown & Liu VSE & 0.000 \\
\hline
\end{tabular}




\begin{tabular}{|l|l|l|r|}
\hline Notch Method & Cycle Count & Fatigue Criterion & Damage \\
\hline NBR & Wang-Brown & Liu VSE & 0.000 \\
\hline UNF & Wang-Brown & Liu VSE & 0.000 \\
\hline ESED & Wang-Brown Modified & Liu VSE & 0.000 \\
\hline NBR & Wang-Brown Modified & Liu VSE & 0.000 \\
\hline UNF & Wang-Brown Modified & Liu VSE & 0.000 \\
\hline ESED & Bannantine-Socie & Liu VSE & 0.000 \\
\hline NBR & Bannantine-Socie & Liu VSE & 0.000 \\
\hline UNF & Bannantine-Socie & Liu VSE & 0.000 \\
\hline
\end{tabular}


Table A.3-5: Fatigue damage results for Pin B at $90^{\circ}$ to the neutral axis and at the centre of the fuse groove $(\hat{z}=0)$. Input stresses determined from elastic FEA data.

\begin{tabular}{|c|c|c|c|}
\hline Notch Method & Cycle Count & Fatigue Criterion & Damage \\
\hline ESED & Wang-Brown & KBM & 0.000 \\
\hline NBR & Wang-Brown & KBM & 0.000 \\
\hline UNF & Wang-Brown & KBM & 0.000 \\
\hline ESED & Wang-Brown Modified & KBM & 0.000 \\
\hline NBR & Wang-Brown Modified & KBM & 0.000 \\
\hline UNF & Wang-Brown Modified & KBM & 0.000 \\
\hline ESED & Bannantine-Socie & KBM & 0.000 \\
\hline NBR & Bannantine-Socie & KBM & 0.000 \\
\hline UNF & Bannantine-Socie & KBM & 0.000 \\
\hline ESED & Wang-Brown & FS & 0.000 \\
\hline NBR & Wang-Brown & FS & 0.000 \\
\hline UNF & Wang-Brown & FS & 0.000 \\
\hline ESED & Wang-Brown Modified & FS & 0.000 \\
\hline NBR & Wang-Brown Modified & FS & 0.000 \\
\hline UNF & Wang-Brown Modified & FS & 0.000 \\
\hline ESED & Bannantine-Socie & FS & 0.000 \\
\hline NBR & Bannantine-Socie & FS & 0.000 \\
\hline UNF & Bannantine-Socie & FS & 0.000 \\
\hline ESED & Wang-Brown & SWT & 0.000 \\
\hline NBR & Wang-Brown & SWT & 0.000 \\
\hline UNF & Wang-Brown & SWT & 0.000 \\
\hline ESED & Wang-Brown Modified & SWT & 0.000 \\
\hline NBR & Wang-Brown Modified & SWT & 0.000 \\
\hline UNF & Wang-Brown Modified & SWT & 0.000 \\
\hline ESED & Bannantine-Socie & SWT & 0.000 \\
\hline NBR & Bannantine-Socie & SWT & 0.000 \\
\hline UNF & Bannantine-Socie & SWT & 0.000 \\
\hline ESED & Wang-Brown & Liu VSE & 0.000 \\
\hline NBR & Wang-Brown & Liu VSE & 0.000 \\
\hline UNF & Wang-Brown & Liu VSE & 0.000 \\
\hline ESED & Wang-Brown Modified & Liu VSE & 0.000 \\
\hline NBR & Wang-Brown Modified & Liu VSE & 0.000 \\
\hline UNF & Wang-Brown Modified & Liu VSE & 0.000 \\
\hline ESED & Bannantine-Socie & Liu VSE & 0.000 \\
\hline NBR & Bannantine-Socie & Liu VSE & 0.000 \\
\hline UNF & Bannantine-Socie & Liu VSE & 0.000 \\
\hline
\end{tabular}


Table A.3-6: Fatigue damage results for Pin B at $90^{\circ}$ to the neutral axis and offset $26 \%$ from the centre of the fuse groove $(\hat{z}=0.26)$. Input stresses determined from elastic FEA data.

\begin{tabular}{|c|c|c|c|}
\hline Notch Method & Cycle Count & Fatigue Criterion & Damage \\
\hline ESED & Wang-Brown & KBM & 0.004 \\
\hline NBR & Wang-Brown & KBM & 0.004 \\
\hline UNF & Wang-Brown & KBM & 0.004 \\
\hline ESED & Wang-Brown Modified & KBM & 0.008 \\
\hline NBR & Wang-Brown Modified & KBM & 0.008 \\
\hline UNF & Wang-Brown Modified & KBM & 0.008 \\
\hline ESED & Bannantine-Socie & KBM & 0.005 \\
\hline NBR & Bannantine-Socie & KBM & 0.005 \\
\hline UNF & Bannantine-Socie & $\mathrm{KBM}$ & 0.005 \\
\hline ESED & Wang-Brown & FS & 0.001 \\
\hline NBR & Wang-Brown & FS & 0.001 \\
\hline UNF & Wang-Brown & FS & 0.001 \\
\hline ESED & Wang-Brown Modified & FS & 0.002 \\
\hline NBR & Wang-Brown Modified & FS & 0.002 \\
\hline UNF & Wang-Brown Modified & FS & 0.002 \\
\hline ESED & Bannantine-Socie & FS & 0.001 \\
\hline NBR & Bannantine-Socie & FS & 0.001 \\
\hline UNF & Bannantine-Socie & FS & 0.001 \\
\hline ESED & Wang-Brown & SWT & 0.015 \\
\hline NBR & Wang-Brown & SWT & 0.015 \\
\hline UNF & Wang-Brown & SWT & 0.015 \\
\hline ESED & Wang-Brown Modified & SWT & 0.028 \\
\hline NBR & Wang-Brown Modified & SWT & 0.028 \\
\hline UNF & Wang-Brown Modified & SWT & 0.028 \\
\hline ESED & Bannantine-Socie & SWT & 0.014 \\
\hline NBR & Bannantine-Socie & SWT & 0.014 \\
\hline UNF & Bannantine-Socie & SWT & 0.014 \\
\hline ESED & Wang-Brown & Liu VSE & 0.001 \\
\hline NBR & Wang-Brown & Liu VSE & 0.001 \\
\hline UNF & Wang-Brown & Liu VSE & 0.001 \\
\hline ESED & Wang-Brown Modified & Liu VSE & 0.002 \\
\hline NBR & Wang-Brown Modified & Liu VSE & 0.002 \\
\hline UNF & Wang-Brown Modified & Liu VSE & 0.002 \\
\hline ESED & Bannantine-Socie & Liu VSE & 0.001 \\
\hline NBR & Bannantine-Socie & Liu VSE & 0.001 \\
\hline UNF & Bannantine-Socie & Liu VSE & 0.001 \\
\hline
\end{tabular}


Table A.3-7: Fatigue damage results for Pin B at $90^{\circ}$ to the neutral axis and offset $-23 \%$ from the centre of the fuse groove $(\hat{\mathrm{z}}=-0.23)$. Input stresses determined from elastic FEA data.

\begin{tabular}{|c|c|c|c|}
\hline Notch Method & Cycle Count & Fatigue Criterion & Damage \\
\hline ESED & Wang-Brown & KBM & 0.000 \\
\hline NBR & Wang-Brown & KBM & 0.000 \\
\hline UNF & Wang-Brown & KBM & 0.000 \\
\hline ESED & Wang-Brown Modified & KBM & 0.000 \\
\hline NBR & Wang-Brown Modified & KBM & 0.000 \\
\hline UNF & Wang-Brown Modified & KBM & 0.000 \\
\hline ESED & Bannantine-Socie & KBM & 0.000 \\
\hline NBR & Bannantine-Socie & KBM & 0.000 \\
\hline UNF & Bannantine-Socie & KBM & 0.000 \\
\hline ESED & Wang-Brown & FS & 0.000 \\
\hline NBR & Wang-Brown & FS & 0.000 \\
\hline UNF & Wang-Brown & FS & 0.000 \\
\hline ESED & Wang-Brown Modified & FS & 0.000 \\
\hline NBR & Wang-Brown Modified & FS & 0.000 \\
\hline UNF & Wang-Brown Modified & FS & 0.000 \\
\hline ESED & Bannantine-Socie & FS & 0.000 \\
\hline NBR & Bannantine-Socie & FS & 0.000 \\
\hline UNF & Bannantine-Socie & FS & 0.000 \\
\hline ESED & Wang-Brown & SWT & 0.000 \\
\hline NBR & Wang-Brown & SWT & 0.000 \\
\hline UNF & Wang-Brown & SWT & 0.000 \\
\hline ESED & Wang-Brown Modified & SWT & 0.000 \\
\hline NBR & Wang-Brown Modified & SWT & 0.000 \\
\hline UNF & Wang-Brown Modified & SWT & 0.000 \\
\hline ESED & Bannantine-Socie & SWT & 0.000 \\
\hline NBR & Bannantine-Socie & SWT & 0.000 \\
\hline UNF & Bannantine-Socie & SWT & 0.000 \\
\hline ESED & Wang-Brown & Liu VSE & 0.000 \\
\hline NBR & Wang-Brown & Liu VSE & 0.000 \\
\hline UNF & Wang-Brown & Liu VSE & 0.000 \\
\hline ESED & Wang-Brown Modified & Liu VSE & 0.000 \\
\hline NBR & Wang-Brown Modified & Liu VSE & 0.000 \\
\hline UNF & Wang-Brown Modified & Liu VSE & 0.000 \\
\hline ESED & Bannantine-Socie & Liu VSE & 0.000 \\
\hline NBR & Bannantine-Socie & Liu VSE & 0.000 \\
\hline UNF & Bannantine-Socie & Liu VSE & 0.000 \\
\hline
\end{tabular}


Table A.3-8: Fatigue damage results for Pin B at $-45^{\circ}$ to the neutral axis and at the centre of the fuse groove $(\hat{z}=0)$. Input stresses determined from elastic FEA data.

\begin{tabular}{|c|c|c|c|}
\hline Notch Method & Cycle Count & Fatigue Criterion & Damage \\
\hline ESED & Wang-Brown & KBM & 0.003 \\
\hline NBR & Wang-Brown & KBM & 0.003 \\
\hline UNF & Wang-Brown & KBM & 0.003 \\
\hline ESED & Wang-Brown Modified & KBM & 0.006 \\
\hline NBR & Wang-Brown Modified & KBM & 0.006 \\
\hline UNF & Wang-Brown Modified & KBM & 0.006 \\
\hline ESED & Bannantine-Socie & KBM & 0.003 \\
\hline NBR & Bannantine-Socie & KBM & 0.003 \\
\hline UNF & Bannantine-Socie & KBM & 0.003 \\
\hline ESED & Wang-Brown & FS & 0.002 \\
\hline NBR & Wang-Brown & FS & 0.002 \\
\hline UNF & Wang-Brown & FS & 0.002 \\
\hline ESED & Wang-Brown Modified & FS & 0.004 \\
\hline NBR & Wang-Brown Modified & FS & 0.004 \\
\hline UNF & Wang-Brown Modified & FS & 0.004 \\
\hline ESED & Bannantine-Socie & FS & 0.002 \\
\hline NBR & Bannantine-Socie & FS & 0.002 \\
\hline UNF & Bannantine-Socie & FS & 0.002 \\
\hline ESED & Wang-Brown & SWT & 0.003 \\
\hline NBR & Wang-Brown & SWT & 0.003 \\
\hline UNF & Wang-Brown & SWT & 0.003 \\
\hline ESED & Wang-Brown Modified & SWT & 0.005 \\
\hline NBR & Wang-Brown Modified & SWT & 0.005 \\
\hline UNF & Wang-Brown Modified & SWT & 0.005 \\
\hline ESED & Bannantine-Socie & SWT & 0.003 \\
\hline NBR & Bannantine-Socie & SWT & 0.003 \\
\hline UNF & Bannantine-Socie & SWT & 0.003 \\
\hline ESED & Wang-Brown & Liu VSE & 0.002 \\
\hline NBR & Wang-Brown & Liu VSE & 0.002 \\
\hline UNF & Wang-Brown & Liu VSE & 0.002 \\
\hline ESED & Wang-Brown Modified & Liu VSE & 0.004 \\
\hline NBR & Wang-Brown Modified & Liu VSE & 0.004 \\
\hline UNF & Wang-Brown Modified & Liu VSE & 0.004 \\
\hline ESED & Bannantine-Socie & Liu VSE & 0.002 \\
\hline NBR & Bannantine-Socie & Liu VSE & 0.002 \\
\hline UNF & Bannantine-Socie & Liu VSE & 0.002 \\
\hline
\end{tabular}


Table A.3-9: Fatigue damage results for Pin B at $-45^{\circ}$ to the neutral axis and offset $35 \%$ from the centre of the fuse groove $(\hat{z}=0.35)$. Input stresses determined from elastic FEA data.

\begin{tabular}{|c|c|c|c|}
\hline Notch Method & Cycle Count & Fatigue Criterion & Damage \\
\hline ESED & Wang-Brown & KBM & 0.000 \\
\hline NBR & Wang-Brown & KBM & 0.000 \\
\hline UNF & Wang-Brown & KBM & 0.000 \\
\hline ESED & Wang-Brown Modified & KBM & 0.000 \\
\hline NBR & Wang-Brown Modified & KBM & 0.000 \\
\hline UNF & Wang-Brown Modified & KBM & 0.000 \\
\hline ESED & Bannantine-Socie & KBM & 0.000 \\
\hline NBR & Bannantine-Socie & KBM & 0.000 \\
\hline UNF & Bannantine-Socie & KBM & 0.000 \\
\hline ESED & Wang-Brown & FS & 0.000 \\
\hline NBR & Wang-Brown & FS & 0.000 \\
\hline UNF & Wang-Brown & FS & 0.000 \\
\hline ESED & Wang-Brown Modified & FS & 0.000 \\
\hline NBR & Wang-Brown Modified & FS & 0.000 \\
\hline UNF & Wang-Brown Modified & FS & 0.000 \\
\hline ESED & Bannantine-Socie & FS & 0.000 \\
\hline NBR & Bannantine-Socie & FS & 0.000 \\
\hline UNF & Bannantine-Socie & FS & 0.000 \\
\hline ESED & Wang-Brown & SWT & 0.000 \\
\hline NBR & Wang-Brown & SWT & 0.000 \\
\hline UNF & Wang-Brown & SWT & 0.000 \\
\hline ESED & Wang-Brown Modified & SWT & 0.000 \\
\hline NBR & Wang-Brown Modified & SWT & 0.000 \\
\hline UNF & Wang-Brown Modified & SWT & 0.000 \\
\hline ESED & Bannantine-Socie & SWT & 0.000 \\
\hline NBR & Bannantine-Socie & SWT & 0.000 \\
\hline UNF & Bannantine-Socie & SWT & 0.000 \\
\hline ESED & Wang-Brown & Liu VSE & 0.002 \\
\hline NBR & Wang-Brown & Liu VSE & 0.002 \\
\hline UNF & Wang-Brown & Liu VSE & 0.002 \\
\hline ESED & Wang-Brown Modified & Liu VSE & 0.003 \\
\hline NBR & Wang-Brown Modified & Liu VSE & 0.003 \\
\hline UNF & Wang-Brown Modified & Liu VSE & 0.003 \\
\hline ESED & Bannantine-Socie & Liu VSE & 0.002 \\
\hline NBR & Bannantine-Socie & Liu VSE & 0.002 \\
\hline UNF & Bannantine-Socie & Liu VSE & 0.002 \\
\hline
\end{tabular}


Table A.3-10: Fatigue damage results for Pin B at $-45^{\circ}$ to the neutral axis and offset $21 \%$ from the centre of the fuse groove $(\hat{z}=-0.21)$. Input stresses determined from elastic FEA data.

\begin{tabular}{|c|c|c|c|}
\hline Notch Method & Cycle Count & Fatigue Criterion & Damage \\
\hline ESED & Wang-Brown & KBM & 1.405 \\
\hline NBR & Wang-Brown & KBM & 1.496 \\
\hline UNF & Wang-Brown & KBM & 1.398 \\
\hline ESED & Wang-Brown Modified & KBM & 2.658 \\
\hline NBR & Wang-Brown Modified & KBM & 2.828 \\
\hline UNF & Wang-Brown Modified & KBM & 2.652 \\
\hline ESED & Bannantine-Socie & KBM & 1.316 \\
\hline NBR & Bannantine-Socie & KBM & 1.410 \\
\hline UNF & Bannantine-Socie & KBM & 1.338 \\
\hline ESED & Wang-Brown & FS & 0.236 \\
\hline NBR & Wang-Brown & FS & 0.245 \\
\hline UNF & Wang-Brown & FS & 0.237 \\
\hline ESED & Wang-Brown Modified & FS & 0.447 \\
\hline NBR & Wang-Brown Modified & FS & 0.462 \\
\hline UNF & Wang-Brown Modified & FS & 0.450 \\
\hline ESED & Bannantine-Socie & FS & 0.221 \\
\hline NBR & Bannantine-Socie & FS & 0.225 \\
\hline UNF & Bannantine-Socie & FS & 0.220 \\
\hline ESED & Wang-Brown & SWT & 3.205 \\
\hline NBR & Wang-Brown & SWT & 3.636 \\
\hline UNF & Wang-Brown & SWT & 3.246 \\
\hline ESED & Wang-Brown Modified & SWT & 6.058 \\
\hline NBR & Wang-Brown Modified & SWT & 6.896 \\
\hline UNF & Wang-Brown Modified & SWT & 6.166 \\
\hline ESED & Bannantine-Socie & SWT & 2.942 \\
\hline NBR & Bannantine-Socie & SWT & 3.295 \\
\hline UNF & Bannantine-Socie & SWT & 2.967 \\
\hline ESED & Wang-Brown & Liu VSE & 0.002 \\
\hline NBR & Wang-Brown & Liu VSE & 0.002 \\
\hline UNF & Wang-Brown & Liu VSE & 0.002 \\
\hline ESED & Wang-Brown Modified & Liu VSE & 0.003 \\
\hline NBR & Wang-Brown Modified & Liu VSE & 0.003 \\
\hline UNF & Wang-Brown Modified & Liu VSE & 0.003 \\
\hline ESED & Bannantine-Socie & Liu VSE & 0.002 \\
\hline NBR & Bannantine-Socie & Liu VSE & 0.002 \\
\hline UNF & Bannantine-Socie & Liu VSE & 0.002 \\
\hline
\end{tabular}


Table A.3-11: Fatigue damage results for Pin B at $-90^{\circ}$ to the neutral axis and at the centre of the fuse groove $(\hat{z}=0)$. Input stresses determined from elastic FEA data.

\begin{tabular}{|c|c|c|c|}
\hline Notch Method & Cycle Count & Fatigue Criterion & Damage \\
\hline ESED & Wang-Brown & KBM & 0.001 \\
\hline NBR & Wang-Brown & KBM & 0.001 \\
\hline UNF & Wang-Brown & KBM & 0.001 \\
\hline ESED & Wang-Brown Modified & KBM & 0.002 \\
\hline NBR & Wang-Brown Modified & KBM & 0.002 \\
\hline UNF & Wang-Brown Modified & KBM & 0.002 \\
\hline ESED & Bannantine-Socie & KBM & 0.001 \\
\hline NBR & Bannantine-Socie & KBM & 0.001 \\
\hline UNF & Bannantine-Socie & KBM & 0.001 \\
\hline ESED & Wang-Brown & FS & 0.001 \\
\hline NBR & Wang-Brown & FS & 0.001 \\
\hline UNF & Wang-Brown & FS & 0.001 \\
\hline ESED & Wang-Brown Modified & FS & 0.001 \\
\hline NBR & Wang-Brown Modified & FS & 0.001 \\
\hline UNF & Wang-Brown Modified & FS & 0.001 \\
\hline ESED & Bannantine-Socie & FS & 0.001 \\
\hline NBR & Bannantine-Socie & FS & 0.001 \\
\hline UNF & Bannantine-Socie & FS & 0.001 \\
\hline ESED & Wang-Brown & SWT & 0.002 \\
\hline NBR & Wang-Brown & SWT & 0.002 \\
\hline UNF & Wang-Brown & SWT & 0.002 \\
\hline ESED & Wang-Brown Modified & SWT & 0.004 \\
\hline NBR & Wang-Brown Modified & SWT & 0.004 \\
\hline UNF & Wang-Brown Modified & SWT & 0.004 \\
\hline ESED & Bannantine-Socie & SWT & 0.002 \\
\hline NBR & Bannantine-Socie & SWT & 0.002 \\
\hline UNF & Bannantine-Socie & SWT & 0.002 \\
\hline ESED & Wang-Brown & Liu VSE & 0.000 \\
\hline NBR & Wang-Brown & Liu VSE & 0.000 \\
\hline UNF & Wang-Brown & Liu VSE & 0.000 \\
\hline ESED & Wang-Brown Modified & Liu VSE & 0.001 \\
\hline NBR & Wang-Brown Modified & Liu VSE & 0.001 \\
\hline UNF & Wang-Brown Modified & Liu VSE & 0.001 \\
\hline ESED & Bannantine-Socie & Liu VSE & 0.000 \\
\hline NBR & Bannantine-Socie & Liu VSE & 0.000 \\
\hline UNF & Bannantine-Socie & Liu VSE & 0.000 \\
\hline
\end{tabular}


Table A.3-12: Fatigue damage results for Pin B at $-90^{\circ}$ to the neutral axis and offset $26 \%$ from the centre of the fuse groove $(\hat{z}=0.26)$. Input stresses determined from elastic FEA data.

\begin{tabular}{|c|c|c|c|}
\hline Notch Method & Cycle Count & Fatigue Criterion & Damage \\
\hline ESED & Wang-Brown & KBM & 0.000 \\
\hline NBR & Wang-Brown & KBM & 0.000 \\
\hline UNF & Wang-Brown & KBM & 0.000 \\
\hline ESED & Wang-Brown Modified & KBM & 0.000 \\
\hline NBR & Wang-Brown Modified & KBM & 0.000 \\
\hline UNF & Wang-Brown Modified & KBM & 0.000 \\
\hline ESED & Bannantine-Socie & KBM & 0.000 \\
\hline NBR & Bannantine-Socie & KBM & 0.000 \\
\hline UNF & Bannantine-Socie & KBM & 0.000 \\
\hline ESED & Wang-Brown & FS & 0.000 \\
\hline NBR & Wang-Brown & FS & 0.000 \\
\hline UNF & Wang-Brown & FS & 0.000 \\
\hline ESED & Wang-Brown Modified & FS & 0.000 \\
\hline NBR & Wang-Brown Modified & FS & 0.000 \\
\hline UNF & Wang-Brown Modified & FS & 0.000 \\
\hline ESED & Bannantine-Socie & FS & 0.000 \\
\hline NBR & Bannantine-Socie & FS & 0.000 \\
\hline UNF & Bannantine-Socie & FS & 0.000 \\
\hline ESED & Wang-Brown & SWT & 0.000 \\
\hline NBR & Wang-Brown & SWT & 0.000 \\
\hline UNF & Wang-Brown & SWT & 0.000 \\
\hline ESED & Wang-Brown Modified & SWT & 0.000 \\
\hline NBR & Wang-Brown Modified & SWT & 0.000 \\
\hline UNF & Wang-Brown Modified & SWT & 0.000 \\
\hline ESED & Bannantine-Socie & SWT & 0.000 \\
\hline NBR & Bannantine-Socie & SWT & 0.000 \\
\hline UNF & Bannantine-Socie & SWT & 0.000 \\
\hline ESED & Wang-Brown & Liu VSE & 0.000 \\
\hline NBR & Wang-Brown & Liu VSE & 0.000 \\
\hline UNF & Wang-Brown & Liu VSE & 0.000 \\
\hline ESED & Wang-Brown Modified & Liu VSE & 0.000 \\
\hline NBR & Wang-Brown Modified & Liu VSE & 0.000 \\
\hline UNF & Wang-Brown Modified & Liu VSE & 0.000 \\
\hline ESED & Bannantine-Socie & Liu VSE & 0.000 \\
\hline NBR & Bannantine-Socie & Liu VSE & 0.000 \\
\hline UNF & Bannantine-Socie & Liu VSE & 0.000 \\
\hline
\end{tabular}


Table A.3-13: Fatigue damage results for Pin B at $-90^{\circ}$ to the neutral axis and offset $-23 \%$ from the centre of the fuse groove $(\hat{z}=-0.23)$. Input stresses determined from elastic FEA data.

\begin{tabular}{|c|c|c|c|}
\hline Notch Method & Cycle Count & Fatigue Criterion & Damage \\
\hline ESED & Wang-Brown & KBM & 2.550 \\
\hline NBR & Wang-Brown & KBM & 2.749 \\
\hline UNF & Wang-Brown & KBM & 2.519 \\
\hline ESED & Wang-Brown Modified & KBM & 4.720 \\
\hline NBR & Wang-Brown Modified & KBM & 5.051 \\
\hline UNF & Wang-Brown Modified & KBM & 4.656 \\
\hline ESED & Bannantine-Socie & KBM & 2.509 \\
\hline NBR & Bannantine-Socie & KBM & 2.687 \\
\hline UNF & Bannantine-Socie & KBM & 2.472 \\
\hline ESED & Wang-Brown & FS & 0.597 \\
\hline NBR & Wang-Brown & FS & 0.624 \\
\hline UNF & Wang-Brown & $\mathrm{FS}$ & 0.599 \\
\hline ESED & Wang-Brown Modified & FS & 1.107 \\
\hline NBR & Wang-Brown Modified & $\mathrm{FS}$ & 1.144 \\
\hline UNF & Wang-Brown Modified & FS & 1.109 \\
\hline ESED & Bannantine-Socie & FS & 0.559 \\
\hline NBR & Bannantine-Socie & $\mathrm{FS}$ & 0.573 \\
\hline UNF & Bannantine-Socie & FS & 0.559 \\
\hline ESED & Wang-Brown & SWT & 8.633 \\
\hline NBR & Wang-Brown & SWT & 9.929 \\
\hline UNF & Wang-Brown & SWT & 8.745 \\
\hline ESED & Wang-Brown Modified & SWT & 15.984 \\
\hline NBR & Wang-Brown Modified & SWT & 18.344 \\
\hline UNF & Wang-Brown Modified & SWT & 16.189 \\
\hline ESED & Bannantine-Socie & SWT & 7.881 \\
\hline NBR & Bannantine-Socie & SWT & 8.995 \\
\hline UNF & Bannantine-Socie & SWT & 7.980 \\
\hline ESED & Wang-Brown & Liu VSE & 1.019 \\
\hline NBR & Wang-Brown & Liu VSE & 1.032 \\
\hline UNF & Wang-Brown & Liu VSE & 1.020 \\
\hline ESED & Wang-Brown Modified & Liu VSE & 1.895 \\
\hline NBR & Wang-Brown Modified & Liu VSE & 1.912 \\
\hline UNF & Wang-Brown Modified & Liu VSE & 1.897 \\
\hline ESED & Bannantine-Socie & Liu VSE & 0.946 \\
\hline NBR & Bannantine-Socie & Liu VSE & 0.952 \\
\hline UNF & Bannantine-Socie & Liu VSE & 0.947 \\
\hline
\end{tabular}




\section{A.4 Pin C FEA-Based Fatigue Damage Results}

Table A.4-1: Fatigue damage results for Pin $\mathrm{C}$ at the neutral axis and centre of the fuse groove $(\hat{\mathrm{z}}$ $=0)$. Input stresses determined from elastic FEA data.

\begin{tabular}{|c|c|c|c|}
\hline Notch Method & Cycle Count & Fatigue Criterion & Damage \\
\hline ESED & Wang-Brown & KBM & 0.003 \\
\hline NBR & Wang-Brown & KBM & 0.003 \\
\hline UNF & Wang-Brown & KBM & 0.003 \\
\hline ESED & Wang-Brown Modified & KBM & 0.0058 \\
\hline NBR & Wang-Brown Modified & KBM & 0.0058 \\
\hline UNF & Wang-Brown Modified & KBM & 0.0058 \\
\hline ESED & Bannantine-Socie & KBM & 0.0624 \\
\hline NBR & Bannantine-Socie & KBM & 0.0624 \\
\hline UNF & Bannantine-Socie & KBM & 0.0624 \\
\hline ESED & Wang-Brown & FS & 0.0349 \\
\hline NBR & Wang-Brown & FS & 0.0349 \\
\hline UNF & Wang-Brown & FS & 0.0349 \\
\hline ESED & Wang-Brown Modified & FS & 0.0675 \\
\hline NBR & Wang-Brown Modified & FS & 0.0675 \\
\hline UNF & Wang-Brown Modified & FS & 0.0675 \\
\hline ESED & Bannantine-Socie & FS & 0.0853 \\
\hline NBR & Bannantine-Socie & FS & 0.0853 \\
\hline UNF & Bannantine-Socie & FS & 0.0853 \\
\hline ESED & Wang-Brown & SWT & 0 \\
\hline NBR & Wang-Brown & SWT & 0 \\
\hline UNF & Wang-Brown & SWT & 0 \\
\hline ESED & Wang-Brown Modified & SWT & 0 \\
\hline NBR & Wang-Brown Modified & SWT & 0 \\
\hline UNF & Wang-Brown Modified & SWT & 0 \\
\hline ESED & Bannantine-Socie & SWT & 0 \\
\hline NBR & Bannantine-Socie & SWT & 0 \\
\hline UNF & Bannantine-Socie & SWT & 0 \\
\hline ESED & Wang-Brown & Liu VSE & 0.115 \\
\hline NBR & Wang-Brown & Liu VSE & 0.115 \\
\hline UNF & Wang-Brown & Liu VSE & 0.115 \\
\hline ESED & Wang-Brown Modified & Liu VSE & 0.2221 \\
\hline NBR & Wang-Brown Modified & Liu VSE & 0.2221 \\
\hline UNF & Wang-Brown Modified & Liu VSE & 0.2221 \\
\hline ESED & Bannantine-Socie & Liu VSE & 0.1145 \\
\hline NBR & Bannantine-Socie & Liu VSE & 0.1145 \\
\hline UNF & Bannantine-Socie & Liu VSE & 0.1145 \\
\hline
\end{tabular}


Table A.4-2: Fatigue damage results for Pin $\mathrm{C}$ at $45^{\circ}$ to the neutral axis and at the centre of the fuse groove $(\hat{z}=0)$. Input stresses determined from elastic FEA data.

\begin{tabular}{|c|c|c|c|}
\hline Notch Method & Cycle Count & Fatigue Criterion & Damage \\
\hline ESED & Wang-Brown & KBM & 0 \\
\hline NBR & Wang-Brown & KBM & 0 \\
\hline UNF & Wang-Brown & KBM & 0 \\
\hline ESED & Wang-Brown Modified & KBM & 0 \\
\hline NBR & Wang-Brown Modified & KBM & 0 \\
\hline UNF & Wang-Brown Modified & KBM & 0 \\
\hline ESED & Bannantine-Socie & KBM & 0.0001 \\
\hline NBR & Bannantine-Socie & KBM & 0.0001 \\
\hline UNF & Bannantine-Socie & KBM & 0.0001 \\
\hline ESED & Wang-Brown & FS & 0 \\
\hline NBR & Wang-Brown & FS & 0 \\
\hline UNF & Wang-Brown & FS & 0 \\
\hline ESED & Wang-Brown Modified & FS & 0 \\
\hline NBR & Wang-Brown Modified & FS & 0 \\
\hline UNF & Wang-Brown Modified & FS & 0 \\
\hline ESED & Bannantine-Socie & FS & 0.0001 \\
\hline NBR & Bannantine-Socie & FS & 0.0001 \\
\hline UNF & Bannantine-Socie & FS & 0.0001 \\
\hline ESED & Wang-Brown & SWT & 0 \\
\hline NBR & Wang-Brown & SWT & 0 \\
\hline UNF & Wang-Brown & SWT & 0 \\
\hline ESED & Wang-Brown Modified & SWT & 0 \\
\hline NBR & Wang-Brown Modified & SWT & 0 \\
\hline UNF & Wang-Brown Modified & SWT & 0 \\
\hline ESED & Bannantine-Socie & SWT & 0 \\
\hline NBR & Bannantine-Socie & SWT & 0 \\
\hline UNF & Bannantine-Socie & SWT & 0 \\
\hline ESED & Wang-Brown & Liu VSE & 0 \\
\hline NBR & Wang-Brown & Liu VSE & 0 \\
\hline UNF & Wang-Brown & Liu VSE & 0 \\
\hline ESED & Wang-Brown Modified & Liu VSE & 0.0001 \\
\hline NBR & Wang-Brown Modified & Liu VSE & 0.0001 \\
\hline UNF & Wang-Brown Modified & Liu VSE & 0.0001 \\
\hline ESED & Bannantine-Socie & Liu VSE & 0 \\
\hline NBR & Bannantine-Socie & Liu VSE & 0 \\
\hline UNF & Bannantine-Socie & Liu VSE & 0 \\
\hline
\end{tabular}


Table A.4-3: Fatigue damage results for Pin $\mathrm{C}$ at $45^{\circ}$ to the neutral axis and offset $28 \%$ from the centre of the fuse groove $(\hat{z}=0.28)$. Input stresses determined from elastic FEA data.

\begin{tabular}{|c|c|c|c|}
\hline Notch Method & Cycle Count & Fatigue Criterion & Damage \\
\hline ESED & Wang-Brown & KBM & 0 \\
\hline NBR & Wang-Brown & KBM & 0 \\
\hline UNF & Wang-Brown & KBM & 0 \\
\hline ESED & Wang-Brown Modified & KBM & 0.0001 \\
\hline NBR & Wang-Brown Modified & KBM & 0.0001 \\
\hline UNF & Wang-Brown Modified & KBM & 0.0001 \\
\hline ESED & Bannantine-Socie & KBM & 0.0033 \\
\hline NBR & Bannantine-Socie & KBM & 0.0035 \\
\hline UNF & Bannantine-Socie & KBM & 0.0035 \\
\hline ESED & Wang-Brown & FS & 0.0001 \\
\hline NBR & Wang-Brown & FS & 0.0001 \\
\hline UNF & Wang-Brown & FS & 0.0001 \\
\hline ESED & Wang-Brown Modified & FS & 0.0002 \\
\hline NBR & Wang-Brown Modified & FS & 0.0002 \\
\hline UNF & Wang-Brown Modified & FS & 0.0002 \\
\hline ESED & Bannantine-Socie & FS & 0.0003 \\
\hline NBR & Bannantine-Socie & FS & 0.0003 \\
\hline UNF & Bannantine-Socie & FS & 0.0003 \\
\hline ESED & Wang-Brown & SWT & 0.0003 \\
\hline NBR & Wang-Brown & SWT & 0.0003 \\
\hline UNF & Wang-Brown & SWT & 0.0003 \\
\hline ESED & Wang-Brown Modified & SWT & 0.0005 \\
\hline NBR & Wang-Brown Modified & SWT & 0.0005 \\
\hline UNF & Wang-Brown Modified & SWT & 0.0005 \\
\hline ESED & Bannantine-Socie & SWT & 0.0003 \\
\hline NBR & Bannantine-Socie & SWT & 0.0003 \\
\hline UNF & Bannantine-Socie & SWT & 0.0003 \\
\hline ESED & Wang-Brown & Liu VSE & 0.0001 \\
\hline NBR & Wang-Brown & Liu VSE & 0.0001 \\
\hline UNF & Wang-Brown & Liu VSE & 0.0001 \\
\hline ESED & Wang-Brown Modified & Liu VSE & 0.0003 \\
\hline NBR & Wang-Brown Modified & Liu VSE & 0.0003 \\
\hline UNF & Wang-Brown Modified & Liu VSE & 0.0003 \\
\hline ESED & Bannantine-Socie & Liu VSE & 0.0001 \\
\hline NBR & Bannantine-Socie & Liu VSE & 0.0001 \\
\hline UNF & Bannantine-Socie & Liu VSE & 0.0001 \\
\hline
\end{tabular}


Table A.4-4: Fatigue damage results for Pin $\mathrm{C}$ at $45^{\circ}$ to the neutral axis and offset $28 \%$ from the centre of the fuse groove $(\hat{z}=-0.28)$. Input stresses determined from elastic FEA data.

\begin{tabular}{|c|c|c|c|}
\hline Notch Method & Cycle Count & Fatigue Criterion & Damage \\
\hline ESED & Wang-Brown & KBM & 0.0009 \\
\hline NBR & Wang-Brown & KBM & 0.0009 \\
\hline UNF & Wang-Brown & KBM & 0.0009 \\
\hline ESED & Wang-Brown Modified & KBM & 0.0017 \\
\hline NBR & Wang-Brown Modified & KBM & 0.0017 \\
\hline UNF & Wang-Brown Modified & KBM & 0.0017 \\
\hline ESED & Bannantine-Socie & KBM & 0.0009 \\
\hline NBR & Bannantine-Socie & KBM & 0.0009 \\
\hline UNF & Bannantine-Socie & KBM & 0.0009 \\
\hline ESED & Wang-Brown & FS & 0.001 \\
\hline NBR & Wang-Brown & FS & 0.001 \\
\hline UNF & Wang-Brown & FS & 0.001 \\
\hline ESED & Wang-Brown Modified & FS & 0.0019 \\
\hline NBR & Wang-Brown Modified & FS & 0.0019 \\
\hline UNF & Wang-Brown Modified & FS & 0.0019 \\
\hline ESED & Bannantine-Socie & FS & 0.0011 \\
\hline NBR & Bannantine-Socie & FS & 0.0011 \\
\hline UNF & Bannantine-Socie & FS & 0.0011 \\
\hline ESED & Wang-Brown & SWT & 0.0002 \\
\hline NBR & Wang-Brown & SWT & 0.0002 \\
\hline UNF & Wang-Brown & SWT & 0.0002 \\
\hline ESED & Wang-Brown Modified & SWT & 0.0004 \\
\hline NBR & Wang-Brown Modified & SWT & 0.0004 \\
\hline UNF & Wang-Brown Modified & SWT & 0.0004 \\
\hline ESED & Bannantine-Socie & SWT & 0.0002 \\
\hline NBR & Bannantine-Socie & SWT & 0.0002 \\
\hline UNF & Bannantine-Socie & SWT & 0.0002 \\
\hline ESED & Wang-Brown & Liu VSE & 0.0033 \\
\hline NBR & Wang-Brown & Liu VSE & 0.0033 \\
\hline UNF & Wang-Brown & Liu VSE & 0.0033 \\
\hline ESED & Wang-Brown Modified & Liu VSE & 0.0061 \\
\hline NBR & Wang-Brown Modified & Liu VSE & 0.0061 \\
\hline UNF & Wang-Brown Modified & Liu VSE & 0.0061 \\
\hline ESED & Bannantine-Socie & Liu VSE & 0.0033 \\
\hline NBR & Bannantine-Socie & Liu VSE & 0.0033 \\
\hline UNF & Bannantine-Socie & Liu VSE & 0.0033 \\
\hline
\end{tabular}


Table A.4-5: Fatigue damage results for Pin $\mathrm{C}$ at $90^{\circ}$ to the neutral axis and at the centre of the fuse groove $(\hat{z}=0)$. Input stresses determined from elastic FEA data.

\begin{tabular}{|c|c|c|c|}
\hline Notch Method & Cycle Count & Fatigue Criterion & Damage \\
\hline ESED & Wang-Brown & KBM & 0 \\
\hline NBR & Wang-Brown & KBM & 0 \\
\hline UNF & Wang-Brown & KBM & 0 \\
\hline ESED & Wang-Brown Modified & KBM & 0 \\
\hline NBR & Wang-Brown Modified & KBM & 0 \\
\hline UNF & Wang-Brown Modified & KBM & 0 \\
\hline ESED & Bannantine-Socie & KBM & 0 \\
\hline NBR & Bannantine-Socie & KBM & 0 \\
\hline UNF & Bannantine-Socie & KBM & 0 \\
\hline ESED & Wang-Brown & FS & 0 \\
\hline NBR & Wang-Brown & FS & 0 \\
\hline UNF & Wang-Brown & FS & 0 \\
\hline ESED & Wang-Brown Modified & FS & 0 \\
\hline NBR & Wang-Brown Modified & $\mathrm{FS}$ & 0 \\
\hline UNF & Wang-Brown Modified & FS & 0 \\
\hline ESED & Bannantine-Socie & FS & 0 \\
\hline NBR & Bannantine-Socie & FS & 0 \\
\hline UNF & Bannantine-Socie & FS & 0 \\
\hline ESED & Wang-Brown & SWT & 0 \\
\hline NBR & Wang-Brown & SWT & 0 \\
\hline UNF & Wang-Brown & SWT & 0 \\
\hline ESED & Wang-Brown Modified & SWT & 0 \\
\hline NBR & Wang-Brown Modified & SWT & 0 \\
\hline UNF & Wang-Brown Modified & SWT & 0 \\
\hline ESED & Bannantine-Socie & SWT & 0 \\
\hline NBR & Bannantine-Socie & SWT & 0 \\
\hline UNF & Bannantine-Socie & SWT & 0 \\
\hline ESED & Wang-Brown & Liu VSE & 0 \\
\hline NBR & Wang-Brown & Liu VSE & 0 \\
\hline UNF & Wang-Brown & Liu VSE & 0 \\
\hline ESED & Wang-Brown Modified & Liu VSE & 0 \\
\hline NBR & Wang-Brown Modified & Liu VSE & 0 \\
\hline UNF & Wang-Brown Modified & Liu VSE & 0 \\
\hline ESED & Bannantine-Socie & Liu VSE & 0 \\
\hline NBR & Bannantine-Socie & Liu VSE & 0 \\
\hline UNF & Bannantine-Socie & Liu VSE & 0 \\
\hline
\end{tabular}


Table A.4-6: Fatigue damage results for Pin $\mathrm{C}$ at $90^{\circ}$ to the neutral axis and offset $30 \%$ from the centre of the fuse groove $(\hat{z}=0.30)$. Input stresses determined from elastic FEA data.

\begin{tabular}{|c|c|c|c|}
\hline Notch Method & Cycle Count & Fatigue Criterion & Damage \\
\hline ESED & Wang-Brown & KBM & 0.0001 \\
\hline NBR & Wang-Brown & KBM & 0.0001 \\
\hline UNF & Wang-Brown & KBM & 0.0001 \\
\hline ESED & Wang-Brown Modified & KBM & 0.0002 \\
\hline NBR & Wang-Brown Modified & KBM & 0.0002 \\
\hline UNF & Wang-Brown Modified & KBM & 0.0002 \\
\hline ESED & Bannantine-Socie & KBM & 0.0001 \\
\hline NBR & Bannantine-Socie & KBM & 0.0001 \\
\hline UNF & Bannantine-Socie & KBM & 0.0001 \\
\hline ESED & Wang-Brown & FS & 0 \\
\hline NBR & Wang-Brown & FS & $\mathrm{C}$ \\
\hline UNF & Wang-Brown & FS & 0 \\
\hline ESED & Wang-Brown Modified & FS & 0.0001 \\
\hline NBR & Wang-Brown Modified & FS & 0.0001 \\
\hline UNF & Wang-Brown Modified & FS & 0.0001 \\
\hline ESED & Bannantine-Socie & FS & 0 \\
\hline NBR & Bannantine-Socie & FS & $\mathrm{C}$ \\
\hline UNF & Bannantine-Socie & FS & $\mathrm{C}$ \\
\hline ESED & Wang-Brown & SWT & 0.0004 \\
\hline NBR & Wang-Brown & SWT & 0.0004 \\
\hline UNF & Wang-Brown & SWT & 0.0004 \\
\hline ESED & Wang-Brown Modified & SWT & 0.0007 \\
\hline NBR & Wang-Brown Modified & SWT & 0.0007 \\
\hline UNF & Wang-Brown Modified & SWT & 0.0007 \\
\hline ESED & Bannantine-Socie & SWT & 0.0004 \\
\hline NBR & Bannantine-Socie & SWT & 0.0004 \\
\hline UNF & Bannantine-Socie & SWT & 0.0004 \\
\hline ESED & Wang-Brown & Liu VSE & $\mathrm{C}$ \\
\hline NBR & Wang-Brown & Liu VSE & 0 \\
\hline UNF & Wang-Brown & Liu VSE & 0 \\
\hline ESED & Wang-Brown Modified & Liu VSE & 0 \\
\hline NBR & Wang-Brown Modified & Liu VSE & 0 \\
\hline UNF & Wang-Brown Modified & Liu VSE & 0 \\
\hline ESED & Bannantine-Socie & Liu VSE & 0 \\
\hline NBR & Bannantine-Socie & Liu VSE & 0 \\
\hline UNF & Bannantine-Socie & Liu VSE & $\mathrm{C}$ \\
\hline
\end{tabular}


Table A.4-7: Fatigue damage results for Pin $\mathrm{C}$ at $90^{\circ}$ to the neutral axis and offset $-30 \%$ from the centre of the fuse groove $(\hat{\mathrm{z}}=-0.30)$. Input stresses determined from elastic FEA data.

\begin{tabular}{|c|c|c|c|}
\hline Notch Method & Cycle Count & Fatigue Criterion & Damage \\
\hline ESED & Wang-Brown & KBM & 0.000 \\
\hline NBR & Wang-Brown & KBM & 0.000 \\
\hline UNF & Wang-Brown & KBM & 0.000 \\
\hline ESED & Wang-Brown Modified & KBM & 0.000 \\
\hline NBR & Wang-Brown Modified & KBM & 0.000 \\
\hline UNF & Wang-Brown Modified & KBM & 0.000 \\
\hline ESED & Bannantine-Socie & KBM & 0.000 \\
\hline NBR & Bannantine-Socie & KBM & 0.000 \\
\hline UNF & Bannantine-Socie & KBM & 0.000 \\
\hline ESED & Wang-Brown & FS & 0.000 \\
\hline NBR & Wang-Brown & FS & 0.000 \\
\hline UNF & Wang-Brown & FS & 0.000 \\
\hline ESED & Wang-Brown Modified & FS & 0.000 \\
\hline NBR & Wang-Brown Modified & FS & 0.000 \\
\hline UNF & Wang-Brown Modified & FS & 0.000 \\
\hline ESED & Bannantine-Socie & FS & 0.000 \\
\hline NBR & Bannantine-Socie & FS & 0.000 \\
\hline UNF & Bannantine-Socie & FS & 0.000 \\
\hline ESED & Wang-Brown & SWT & 0.000 \\
\hline NBR & Wang-Brown & SWT & 0.000 \\
\hline UNF & Wang-Brown & SWT & 0.000 \\
\hline ESED & Wang-Brown Modified & SWT & 0.000 \\
\hline NBR & Wang-Brown Modified & SWT & 0.000 \\
\hline UNF & Wang-Brown Modified & SWT & 0.000 \\
\hline ESED & Bannantine-Socie & SWT & 0.000 \\
\hline NBR & Bannantine-Socie & SWT & 0.000 \\
\hline UNF & Bannantine-Socie & SWT & 0.000 \\
\hline ESED & Wang-Brown & Liu VSE & 0 \\
\hline NBR & Wang-Brown & Liu VSE & 0 \\
\hline UNF & Wang-Brown & Liu VSE & 0 \\
\hline ESED & Wang-Brown Modified & Liu VSE & 0.0001 \\
\hline NBR & Wang-Brown Modified & Liu VSE & 0.0001 \\
\hline UNF & Wang-Brown Modified & Liu VSE & 0.0001 \\
\hline ESED & Bannantine-Socie & Liu VSE & 0 \\
\hline NBR & Bannantine-Socie & Liu VSE & 0 \\
\hline UNF & Bannantine-Socie & Liu VSE & 0 \\
\hline
\end{tabular}


Table A.4-8: Fatigue damage results for Pin $\mathrm{C}$ at $-45^{\circ}$ to the neutral axis and the centre of the fuse groove $(\hat{z}=0)$. Input stresses determined from elastic FEA data.

\begin{tabular}{|c|c|c|c|}
\hline Notch Method & Cycle Count & Fatigue Criterion & Damage \\
\hline ESED & Wang-Brown & KBM & 0.0001 \\
\hline NBR & Wang-Brown & KBM & 0.0001 \\
\hline UNF & Wang-Brown & KBM & 0.0001 \\
\hline ESED & Wang-Brown Modified & KBM & 0.0001 \\
\hline NBR & Wang-Brown Modified & KBM & 0.0001 \\
\hline UNF & Wang-Brown Modified & KBM & 0.0001 \\
\hline ESED & Bannantine-Socie & KBM & 0.0002 \\
\hline NBR & Bannantine-Socie & KBM & 0.0002 \\
\hline UNF & Bannantine-Socie & KBM & 0.0002 \\
\hline ESED & Wang-Brown & FS & 0 \\
\hline NBR & Wang-Brown & FS & 0 \\
\hline UNF & Wang-Brown & FS & 0 \\
\hline ESED & Wang-Brown Modified & FS & 0.0001 \\
\hline NBR & Wang-Brown Modified & FS & 0.0001 \\
\hline UNF & Wang-Brown Modified & FS & 0.0001 \\
\hline ESED & Bannantine-Socie & FS & 0.0001 \\
\hline NBR & Bannantine-Socie & FS & 0.0001 \\
\hline UNF & Bannantine-Socie & FS & 0.0001 \\
\hline ESED & Wang-Brown & SWT & 0 \\
\hline NBR & Wang-Brown & SWT & 0 \\
\hline UNF & Wang-Brown & SWT & 0 \\
\hline ESED & Wang-Brown Modified & SWT & 0 \\
\hline NBR & Wang-Brown Modified & SWT & 0 \\
\hline UNF & Wang-Brown Modified & SWT & 0 \\
\hline ESED & Bannantine-Socie & SWT & 0 \\
\hline NBR & Bannantine-Socie & SWT & 0 \\
\hline UNF & Bannantine-Socie & SWT & 0 \\
\hline ESED & Wang-Brown & Liu VSE & 0 \\
\hline NBR & Wang-Brown & Liu VSE & 0 \\
\hline UNF & Wang-Brown & Liu VSE & 0 \\
\hline ESED & Wang-Brown Modified & Liu VSE & 0.0001 \\
\hline NBR & Wang-Brown Modified & Liu VSE & 0.0001 \\
\hline UNF & Wang-Brown Modified & Liu VSE & 0.0001 \\
\hline ESED & Bannantine-Socie & Liu VSE & 0 \\
\hline NBR & Bannantine-Socie & Liu VSE & 0 \\
\hline UNF & Bannantine-Socie & Liu VSE & 0 \\
\hline
\end{tabular}


Table A.4-9: Fatigue damage results for Pin $\mathrm{C}$ at $-45^{\circ}$ to the neutral axis and offset $28 \%$ from the centre of the fuse groove $(\hat{z}=0.28)$. Input stresses determined from elastic FEA data.

\begin{tabular}{|c|c|c|c|}
\hline Notch Method & Cycle Count & Fatigue Criterion & Damage \\
\hline ESED & Wang-Brown & KBM & 0.003 \\
\hline NBR & Wang-Brown & KBM & 0.003 \\
\hline UNF & Wang-Brown & KBM & 0.003 \\
\hline ESED & Wang-Brown Modified & KBM & 0.005 \\
\hline NBR & Wang-Brown Modified & KBM & 0.005 \\
\hline UNF & Wang-Brown Modified & KBM & 0.005 \\
\hline ESED & Bannantine-Socie & KBM & 0.003 \\
\hline NBR & Bannantine-Socie & KBM & 0.003 \\
\hline UNF & Bannantine-Socie & KBM & 0.003 \\
\hline ESED & Wang-Brown & FS & 0.000 \\
\hline NBR & Wang-Brown & FS & 0.000 \\
\hline UNF & Wang-Brown & FS & 0.000 \\
\hline ESED & Wang-Brown Modified & FS & 0.000 \\
\hline NBR & Wang-Brown Modified & FS & 0.000 \\
\hline UNF & Wang-Brown Modified & FS & 0.000 \\
\hline ESED & Bannantine-Socie & FS & 0.000 \\
\hline NBR & Bannantine-Socie & FS & 0.000 \\
\hline UNF & Bannantine-Socie & FS & 0.000 \\
\hline ESED & Wang-Brown & SWT & 0.000 \\
\hline NBR & Wang-Brown & SWT & 0.000 \\
\hline UNF & Wang-Brown & SWT & 0.000 \\
\hline ESED & Wang-Brown Modified & SWT & 0.000 \\
\hline NBR & Wang-Brown Modified & SWT & 0.000 \\
\hline UNF & Wang-Brown Modified & SWT & 0.000 \\
\hline ESED & Bannantine-Socie & SWT & 0.000 \\
\hline NBR & Bannantine-Socie & SWT & 0.000 \\
\hline UNF & Bannantine-Socie & SWT & 0.000 \\
\hline ESED & Wang-Brown & Liu VSE & 0.000 \\
\hline NBR & Wang-Brown & Liu VSE & 0.000 \\
\hline UNF & Wang-Brown & \begin{tabular}{|l} 
Liu VSE \\
\end{tabular} & 0.000 \\
\hline ESED & Wang-Brown Modified & Liu VSE & 0.000 \\
\hline NBR & Wang-Brown Modified & Liu VSE & 0.000 \\
\hline UNF & Wang-Brown Modified & Liu VSE & 0.000 \\
\hline ESED & Bannantine-Socie & Liu VSE & 0.000 \\
\hline NBR & Bannantine-Socie & Liu VSE & 0.000 \\
\hline UNF & Bannantine-Socie & Liu VSE & 0.000 \\
\hline
\end{tabular}


Table A.4-10: Fatigue damage results for Pin $\mathrm{C}$ at $-45^{\circ}$ to the neutral axis and offset $-28 \%$ from the centre of the fuse groove $(\hat{\mathrm{z}}=-0.28)$. Input stresses determined from elastic FEA data.

\begin{tabular}{|c|c|c|c|}
\hline Notch Method & Cycle Count & Fatigue Criterion & Damage \\
\hline ESED & Wang-Brown & KBM & 0.0001 \\
\hline NBR & Wang-Brown & KBM & 0.0001 \\
\hline UNF & Wang-Brown & KBM & 0.0001 \\
\hline ESED & Wang-Brown Modified & KBM & 0.0002 \\
\hline NBR & Wang-Brown Modified & KBM & 0.0002 \\
\hline UNF & Wang-Brown Modified & KBM & 0.0002 \\
\hline ESED & Bannantine-Socie & KBM & 0.0016 \\
\hline NBR & Bannantine-Socie & KBM & 0.0016 \\
\hline UNF & Bannantine-Socie & KBM & 0.0016 \\
\hline ESED & Wang-Brown & FS & 0.0007 \\
\hline NBR & Wang-Brown & FS & 0.0007 \\
\hline UNF & Wang-Brown & FS & 0.0007 \\
\hline ESED & Wang-Brown Modified & FS & 0.0013 \\
\hline NBR & Wang-Brown Modified & $\mathrm{FS}$ & 0.0013 \\
\hline UNF & Wang-Brown Modified & FS & 0.0013 \\
\hline ESED & Bannantine-Socie & FS & 0.0014 \\
\hline NBR & Bannantine-Socie & FS & 0.0014 \\
\hline UNF & Bannantine-Socie & FS & 0.0014 \\
\hline ESED & Wang-Brown & SWT & 0.0005 \\
\hline NBR & Wang-Brown & SWT & 0.0005 \\
\hline UNF & Wang-Brown & SWT & 0.0005 \\
\hline ESED & Wang-Brown Modified & SWT & 0.0009 \\
\hline NBR & Wang-Brown Modified & SWT & 0.0009 \\
\hline UNF & Wang-Brown Modified & SWT & 0.0009 \\
\hline ESED & Bannantine-Socie & SWT & 0.0005 \\
\hline NBR & Bannantine-Socie & SWT & 0.0005 \\
\hline UNF & Bannantine-Socie & SWT & 0.0005 \\
\hline ESED & Wang-Brown & Liu VSE & 0.0028 \\
\hline NBR & Wang-Brown & Liu VSE & 0.0028 \\
\hline UNF & Wang-Brown & Liu VSE & 0.0028 \\
\hline ESED & Wang-Brown Modified & Liu VSE & 0.0054 \\
\hline NBR & Wang-Brown Modified & Liu VSE & 0.0054 \\
\hline UNF & Wang-Brown Modified & Liu VSE & 0.0054 \\
\hline ESED & Bannantine-Socie & Liu VSE & 0.0029 \\
\hline NBR & Bannantine-Socie & Liu VSE & 0.0029 \\
\hline UNF & Bannantine-Socie & Liu VSE & 0.0029 \\
\hline
\end{tabular}


Table A.4-11: Fatigue damage results for Pin $\mathrm{C}$ at $-90^{\circ}$ to the neutral axis and at the centre of the fuse groove $(\hat{z}=0)$. Input stresses determined from elastic FEA data.

\begin{tabular}{|c|c|c|c|}
\hline Notch Method & Cycle Count & Fatigue Criterion & Damage \\
\hline ESED & Wang-Brown & KBM & 0.000 \\
\hline NBR & Wang-Brown & KBM & 0.000 \\
\hline UNF & Wang-Brown & KBM & 0.000 \\
\hline ESED & Wang-Brown Modified & KBM & 0.000 \\
\hline NBR & Wang-Brown Modified & KBM & 0.000 \\
\hline UNF & Wang-Brown Modified & KBM & 0.000 \\
\hline ESED & Bannantine-Socie & KBM & 0.000 \\
\hline NBR & Bannantine-Socie & KBM & 0.000 \\
\hline UNF & Bannantine-Socie & KBM & 0.000 \\
\hline ESED & Wang-Brown & FS & 0.000 \\
\hline NBR & Wang-Brown & FS & 0.000 \\
\hline UNF & Wang-Brown & FS & 0.000 \\
\hline ESED & Wang-Brown Modified & FS & 0.000 \\
\hline NBR & Wang-Brown Modified & FS & 0.000 \\
\hline UNF & Wang-Brown Modified & FS & 0.000 \\
\hline ESED & Bannantine-Socie & FS & 0.000 \\
\hline NBR & Bannantine-Socie & FS & 0.000 \\
\hline UNF & Bannantine-Socie & FS & 0.000 \\
\hline ESED & Wang-Brown & SWT & 0.000 \\
\hline NBR & Wang-Brown & SWT & 0.000 \\
\hline UNF & Wang-Brown & SWT & 0.000 \\
\hline ESED & Wang-Brown Modified & SWT & 0.000 \\
\hline NBR & Wang-Brown Modified & SWT & 0.000 \\
\hline UNF & Wang-Brown Modified & SWT & 0.000 \\
\hline ESED & Bannantine-Socie & SWT & 0.000 \\
\hline NBR & Bannantine-Socie & SWT & 0.000 \\
\hline UNF & Bannantine-Socie & SWT & 0.000 \\
\hline ESED & Wang-Brown & Liu VSE & 0.000 \\
\hline NBR & Wang-Brown & Liu VSE & 0.000 \\
\hline UNF & Wang-Brown & Liu VSE & 0.000 \\
\hline ESED & Wang-Brown Modified & Liu VSE & 0.000 \\
\hline NBR & Wang-Brown Modified & Liu VSE & 0.000 \\
\hline UNF & Wang-Brown Modified & Liu VSE & 0.000 \\
\hline ESED & Bannantine-Socie & Liu VSE & 0.000 \\
\hline NBR & Bannantine-Socie & Liu VSE & 0.000 \\
\hline UNF & Bannantine-Socie & Liu VSE & 0.000 \\
\hline
\end{tabular}


Table A.4-12: Fatigue damage results for Pin $\mathrm{C}$ at $-90^{\circ}$ to the neutral axis and offset $30 \%$ from the centre of the fuse groove $(\hat{z}=0.30)$. Input stresses determined from elastic FEA data.

\begin{tabular}{|c|c|c|c|}
\hline Notch Method & Cycle Count & Fatigue Criterion & Damage \\
\hline ESED & Wang-Brown & KBM & 0.000 \\
\hline NBR & Wang-Brown & KBM & 0.000 \\
\hline UNF & Wang-Brown & KBM & 0.000 \\
\hline ESED & Wang-Brown Modified & KBM & 0.000 \\
\hline NBR & Wang-Brown Modified & KBM & 0.000 \\
\hline UNF & Wang-Brown Modified & KBM & 0.000 \\
\hline ESED & Bannantine-Socie & KBM & 0.000 \\
\hline NBR & Bannantine-Socie & KBM & 0.000 \\
\hline UNF & Bannantine-Socie & KBM & 0.000 \\
\hline ESED & Wang-Brown & FS & 0.000 \\
\hline NBR & Wang-Brown & FS & 0.000 \\
\hline UNF & Wang-Brown & FS & 0.000 \\
\hline ESED & Wang-Brown Modified & FS & 0.000 \\
\hline NBR & Wang-Brown Modified & FS & 0.000 \\
\hline UNF & Wang-Brown Modified & FS & 0.000 \\
\hline ESED & Bannantine-Socie & FS & 0.000 \\
\hline NBR & Bannantine-Socie & FS & 0.000 \\
\hline UNF & Bannantine-Socie & FS & 0.000 \\
\hline ESED & Wang-Brown & SWT & 0.000 \\
\hline NBR & Wang-Brown & SWT & 0.000 \\
\hline UNF & Wang-Brown & SWT & 0.000 \\
\hline ESED & Wang-Brown Modified & SWT & 0.000 \\
\hline NBR & Wang-Brown Modified & SWT & 0.000 \\
\hline UNF & Wang-Brown Modified & SWT & 0.000 \\
\hline ESED & Bannantine-Socie & SWT & 0.000 \\
\hline NBR & Bannantine-Socie & SWT & 0.000 \\
\hline UNF & Bannantine-Socie & SWT & 0.000 \\
\hline ESED & Wang-Brown & Liu VSE & 0.001 \\
\hline NBR & Wang-Brown & Liu VSE & 0.001 \\
\hline UNF & Wang-Brown & Liu VSE & 0.001 \\
\hline ESED & Wang-Brown Modified & Liu VSE & 0.000 \\
\hline NBR & Wang-Brown Modified & Liu VSE & 0.000 \\
\hline UNF & Wang-Brown Modified & Liu VSE & 0.000 \\
\hline ESED & Bannantine-Socie & Liu VSE & 0.000 \\
\hline NBR & Bannantine-Socie & Liu VSE & 0.000 \\
\hline UNF & Bannantine-Socie & Liu VSE & 0.000 \\
\hline
\end{tabular}


Table A.4-13: Fatigue damage results for Pin $\mathrm{C}$ at $-90^{\circ}$ to the neutral axis and offset $-30 \%$ from the centre of the fuse groove $(\hat{\mathrm{z}}=-0.30)$. Input stresses determined from elastic FEA data.

\begin{tabular}{|c|c|c|c|}
\hline Notch Method & Cycle Count & Fatigue Criterion & Damage \\
\hline ESED & Wang-Brown & KBM & 0.000 \\
\hline NBR & Wang-Brown & KBM & 0.000 \\
\hline UNF & Wang-Brown & KBM & 0.000 \\
\hline ESED & Wang-Brown Modified & KBM & 0.000 \\
\hline NBR & Wang-Brown Modified & KBM & 0.000 \\
\hline UNF & Wang-Brown Modified & KBM & 0.000 \\
\hline ESED & Bannantine-Socie & KBM & 0.000 \\
\hline NBR & Bannantine-Socie & KBM & 0.000 \\
\hline UNF & Bannantine-Socie & KBM & 0.000 \\
\hline ESED & Wang-Brown & FS & 0.000 \\
\hline NBR & Wang-Brown & FS & 0.000 \\
\hline UNF & Wang-Brown & FS & 0.000 \\
\hline ESED & Wang-Brown Modified & FS & 0.000 \\
\hline NBR & Wang-Brown Modified & $\mathrm{FS}$ & 0.000 \\
\hline UNF & Wang-Brown Modified & FS & 0.000 \\
\hline ESED & Bannantine-Socie & FS & 0.000 \\
\hline NBR & Bannantine-Socie & FS & 0.000 \\
\hline UNF & Bannantine-Socie & FS & 0.000 \\
\hline ESED & Wang-Brown & SWT & 0.000 \\
\hline NBR & Wang-Brown & SWT & 0.000 \\
\hline UNF & Wang-Brown & SWT & 0.000 \\
\hline ESED & Wang-Brown Modified & SWT & 0.000 \\
\hline NBR & Wang-Brown Modified & SWT & 0.000 \\
\hline UNF & Wang-Brown Modified & SWT & 0.000 \\
\hline ESED & Bannantine-Socie & SWT & 0.000 \\
\hline NBR & Bannantine-Socie & SWT & 0.000 \\
\hline UNF & Bannantine-Socie & SWT & 0.000 \\
\hline ESED & Wang-Brown & Liu VSE & 0.000 \\
\hline NBR & Wang-Brown & Liu VSE & 0.000 \\
\hline UNF & Wang-Brown & Liu VSE & 0.000 \\
\hline ESED & Wang-Brown Modified & Liu VSE & 0.000 \\
\hline NBR & Wang-Brown Modified & Liu VSE & 0.000 \\
\hline UNF & Wang-Brown Modified & Liu VSE & 0.000 \\
\hline ESED & Bannantine-Socie & Liu VSE & 0.000 \\
\hline NBR & Bannantine-Socie & Liu VSE & 0.000 \\
\hline UNF & Bannantine-Socie & Liu VSE & 0.000 \\
\hline
\end{tabular}




\section{Appendix B}

This appendix provides details regarding the basic operation of Multiaxial Fatigue version 1.0, the multiaxial fatigue analysis program written for this thesis.

\section{B.1 Input Files}

Three input files are required to perform an analysis in Multiaxial Fatigue:

1. The load spectrum file (.csv) that provides the order in which the load cases occur;

2. The stress file (.prn) that provides the stress components for each load case; and

3. The material data file (.xlsx) that provides the material properties and elastic-plastic stress-strain curve.

The load spectrum file is organized by sequences that contain a certain number of load cases, shown in Figure B.1-1. Sequences are used to make it easier to define repeated patterns of load cases. In the program, the load spectrum input file is read in first so that the program knows what load cases to look for when building the stress history file.

The stress input file shown in Figure B.1-2 is organized in a list format with six stress components for each load case. The stresses in this file should be the theoretical elastic stresses used for the notch analysis.

The material data input file is an Excel spreadsheet which contains two sheets to organize the material data. As shown in

Figure B.1-3, the first sheet contains basic elastic and fatigue properties and the second sheet is a table of the elastic-plastic stress-strain curve. 


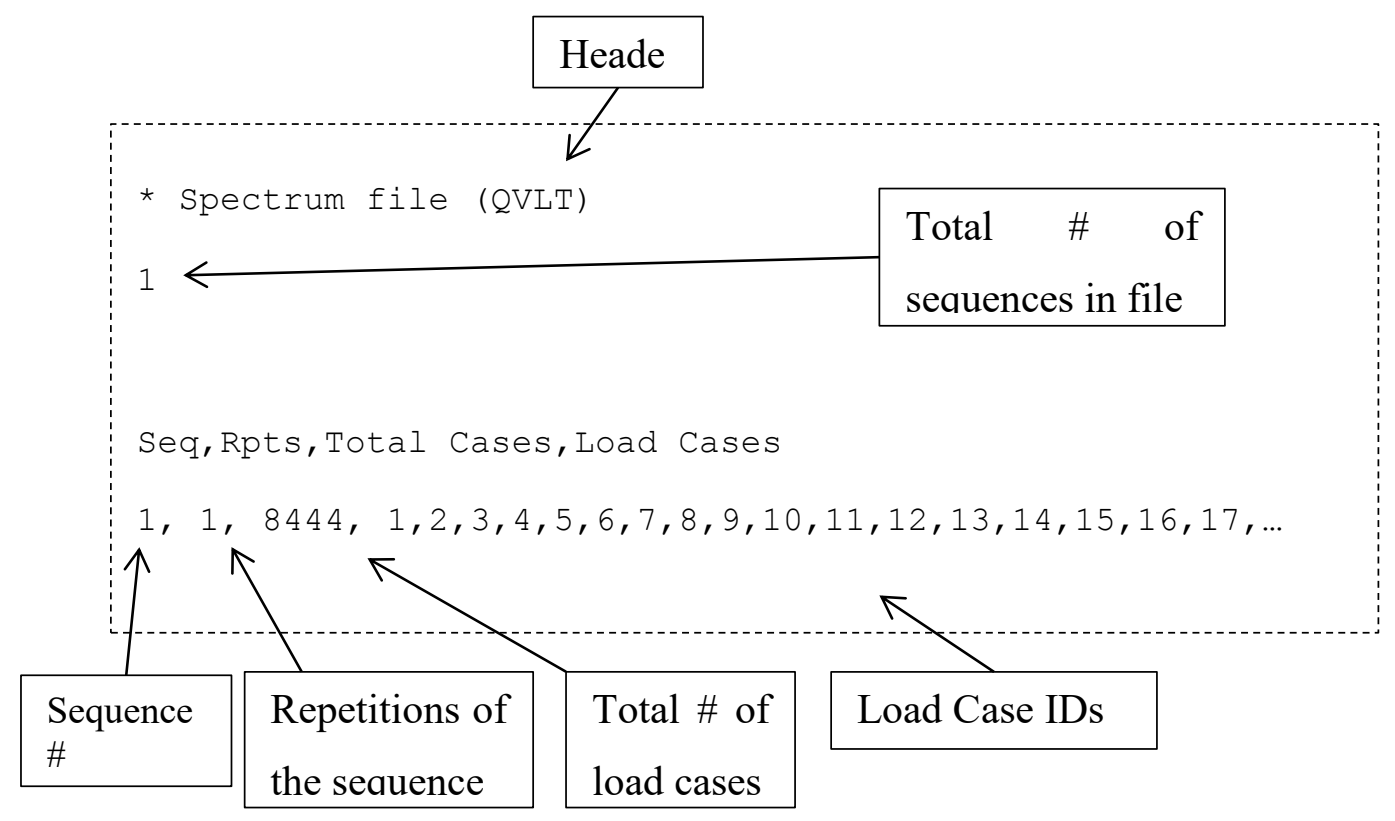

Figure B.1-1: Load spectrum input file format for Multiaxial Fatigue v. 1.0.

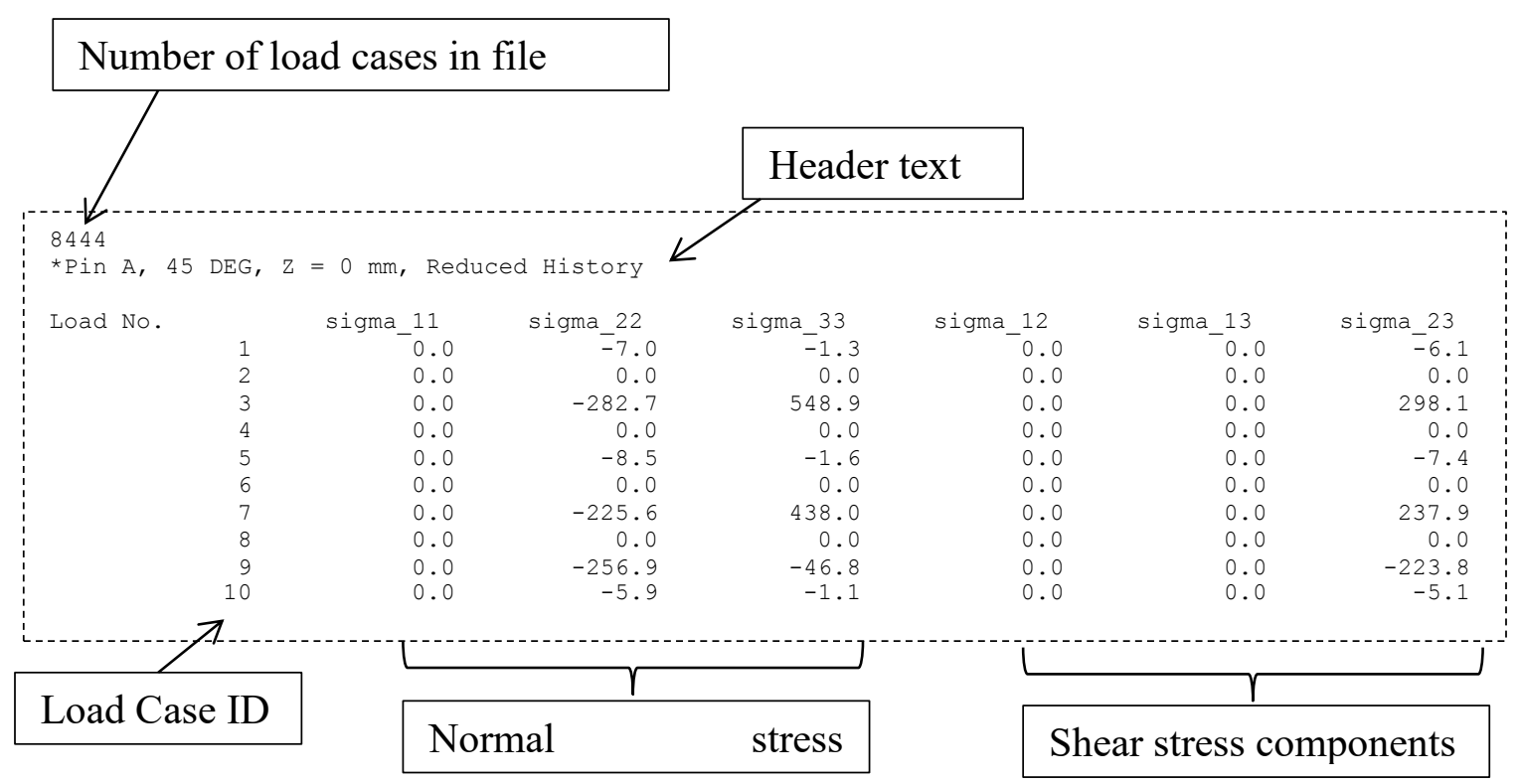

Figure B.1-2: Stress input file format for Multiaxial Fatigue v. 1.0. 


\begin{tabular}{|c|c|c|c|c|}
\hline \multicolumn{5}{|c|}{ Sheet: main } \\
\hline 4 & A & B & C & D \\
\hline \multicolumn{5}{|l|}{1} \\
\hline 2 & & \multirow[t]{2}{*}{ Material: } & \multicolumn{2}{|c|}{ S460N (Ye et al.) } \\
\hline \multicolumn{4}{|c|}{ 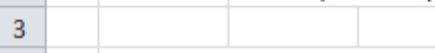 } & \\
\hline 4 & & \multicolumn{3}{|c|}{ Monotonic Material Data: } \\
\hline 5 & & $E=$ & 208500 & $\mathrm{MPa}$ \\
\hline 6 & & $v=$ & 0.3 & \\
\hline 7 & & $\mathrm{~K}=$ & 1115 & $\mathrm{MPa}$ \\
\hline 8 & & $\mathrm{n}=$ & 0.161 & \\
\hline \multicolumn{5}{|l|}{9} \\
\hline 10 & & \multicolumn{3}{|c|}{ Uniaxial Cyclic Properties: } \\
\hline 11 & & $K^{\prime}=$ & 1115 & $\mathrm{MPa}$ \\
\hline 12 & & $n^{\prime}=$ & 0.161 & \\
\hline \multicolumn{5}{|l|}{13} \\
\hline 14 & & \multicolumn{3}{|c|}{ Uniaxial Cyclic Properties: } \\
\hline 15 & & $K^{\prime}=$ & 2460 & \\
\hline 16 & & $n^{\prime}=$ & 0.15 & \\
\hline \multicolumn{5}{|l|}{17} \\
\hline 18 & & \multicolumn{2}{|c|}{ Fatigue constants } & \\
\hline 19 & & $\sigma_{f}^{\prime}=$ & 1500 & $\mathrm{MPa}$ \\
\hline 20 & & $\mathrm{~b}=$ & -0.05 & \\
\hline 21 & & $\varepsilon_{f}^{\prime}=$ & 0.25 & \\
\hline 22 & & $c=$ & -0.6 & \\
\hline$n$ & & & & \\
\hline
\end{tabular}

\begin{tabular}{|c|r|r|r|r|}
\hline \multicolumn{5}{|c|}{ Sheet: sscurve } \\
\hline \hline 1 & A & B & \multicolumn{1}{l|}{ C } & D \\
\hline 1 & & & & \\
\hline 2 & & $\sigma$ & $\varepsilon_{p}$ & \\
\hline 3 & & 500.00 & 0.0000 & \\
\hline 4 & & 700.15 & 0.0015 & \\
\hline 5 & & 760.16 & 0.0025 & \\
\hline 6 & & 802.48 & 0.0035 & \\
\hline 7 & & 835.62 & 0.0045 & \\
\hline 8 & & 863.05 & 0.0055 & \\
\hline 9 & & 886.58 & 0.0065 & \\
\hline 10 & & 907.24 & 0.0075 & \\
\hline 11 & & 925.71 & 0.0085 & \\
\hline 12 & & 942.44 & 0.0095 & \\
\hline 13 & & 957.75 & 0.0105 & \\
\hline 14 & & 971.88 & 0.0115 & \\
\hline 15 & & 985.01 & 0.0125 & \\
\hline 16 & & 997.29 & 0.0135 & \\
\hline 17 & & 1008.83 & 0.0145 & \\
\hline 18 & & 1019.72 & 0.0155 & \\
\hline 19 & & 1030.04 & 0.0165 & \\
\hline 20 & 1039.84 & 0.0175 & \\
\hline & & & \\
\hline
\end{tabular}

Figure B.1-3: Material data input file format for Multiaxial Fatigue v. 1.0. 


\section{B.2 Preferences}

Preference settings are available for the user to change the default settings for the three input files and the output directory for future use. This is shown in Figure B.2-1.

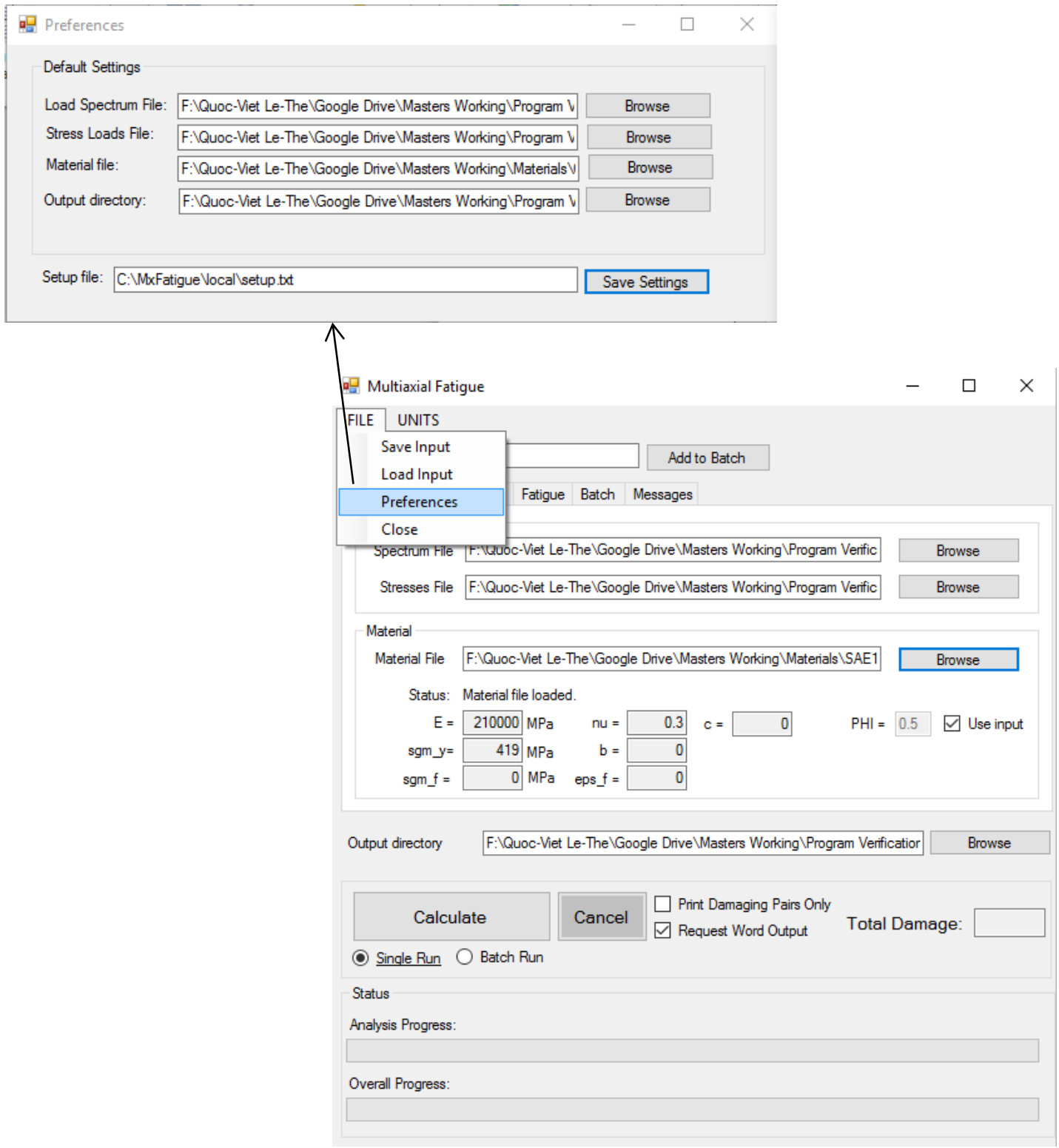

Figure B.2-1: Preferences settings. 


\section{B.3 Analysis Settings}

The main window is divided into three main sections as shown in Figure B.3-1: (a) the analysis settings section, (b) the execution section, and (c) the progress status section. The analysis settings section is further divided into 6 tabs that allow the user to make selections in different aspects of the fatigue analysis. In the Input tab shown in Figure B.3-1, the spectrum, stresses, and material data files can be selected. The file can either be selected using the Browse buttons or the address of the file can be pasted into the text box. In the execution section the output directory can be selected, the user can choose to run a single analysis or run a batch of jobs, the user can choose to filter the cycles that are printed and the user can request for a Word output. At the end of an analysis, the highest cumulative damage is shown in the execution section.

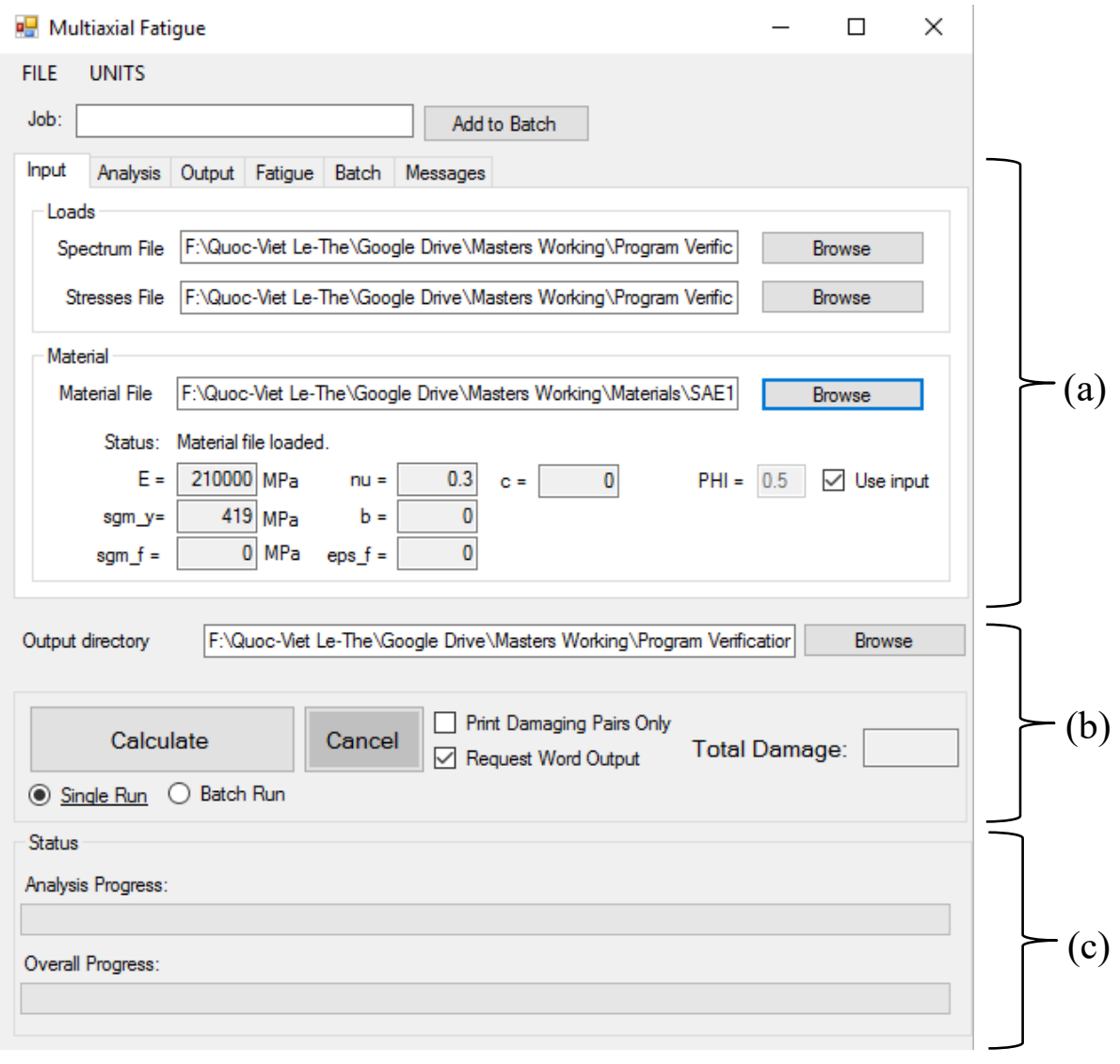

Figure B.3-1: Input file settings. 
The Analysis tab shown in Figure B.3-2 provides the user with options for select the type of notch correction method and for specifying the maximum stress increment. The $2 \mathrm{D}$ Analysis Settings allows the user to control which component of stress will be treated as the out-of-plane stress, which is zeroed throughout the entire fatigue analysis. All analyses performed in Multiaxial Fatigue version 1.0 are plane stress analyses.

The Output tab shown in Figure B.3-3 provides checkboxes for the user to select stress and strain components that they want to have printed in the output files. Figure B.3-4 shows the Fatigue settings tab, which provides options for the user to select the cycle counting procedure and the fatigue damage criterion. If the Bannantine-Socie cycle counting procedure is selected, the user is also required to input the critical angles to be analyzed and the reference strain angle to be used for the rainflow cycle count. Under the Fatigue Controls group box, the user can also set the number of blocks and scatter factor to be applied to the final damage calculation.

If the user wishes to perform a batch analysis, the "Add to Batch" button can be used to add a job to the batch queue with the current selected settings. Once added, the job will appear in the Batch tab shown in Figure B.3-5. With multiple jobs to the queue the user can also save the list of jobs to a local directory for later use. To perform a batch analysis, the "Batch Run" toggle directly below the "Calculate" button must be selected. 


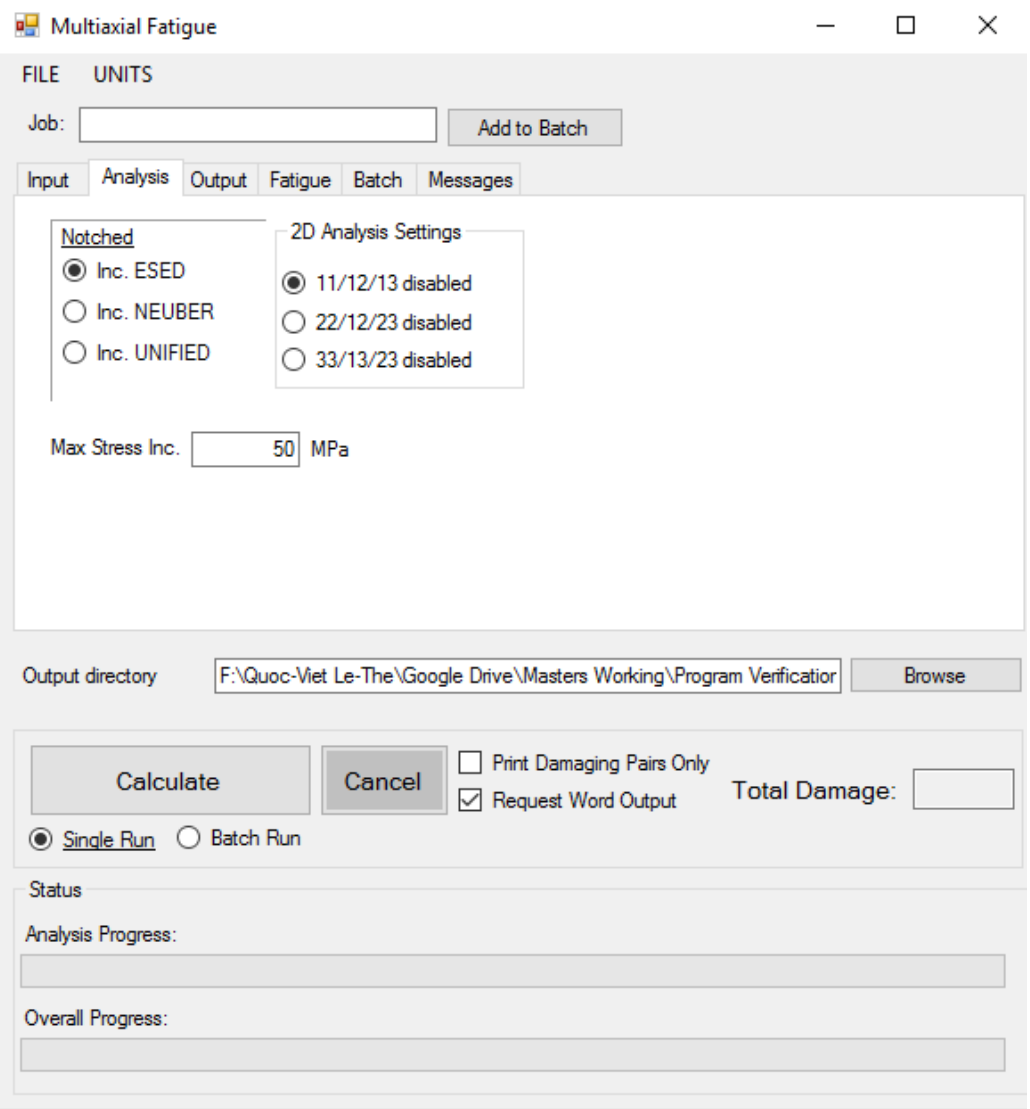

Figure B.3-2: Analysis type settings. 


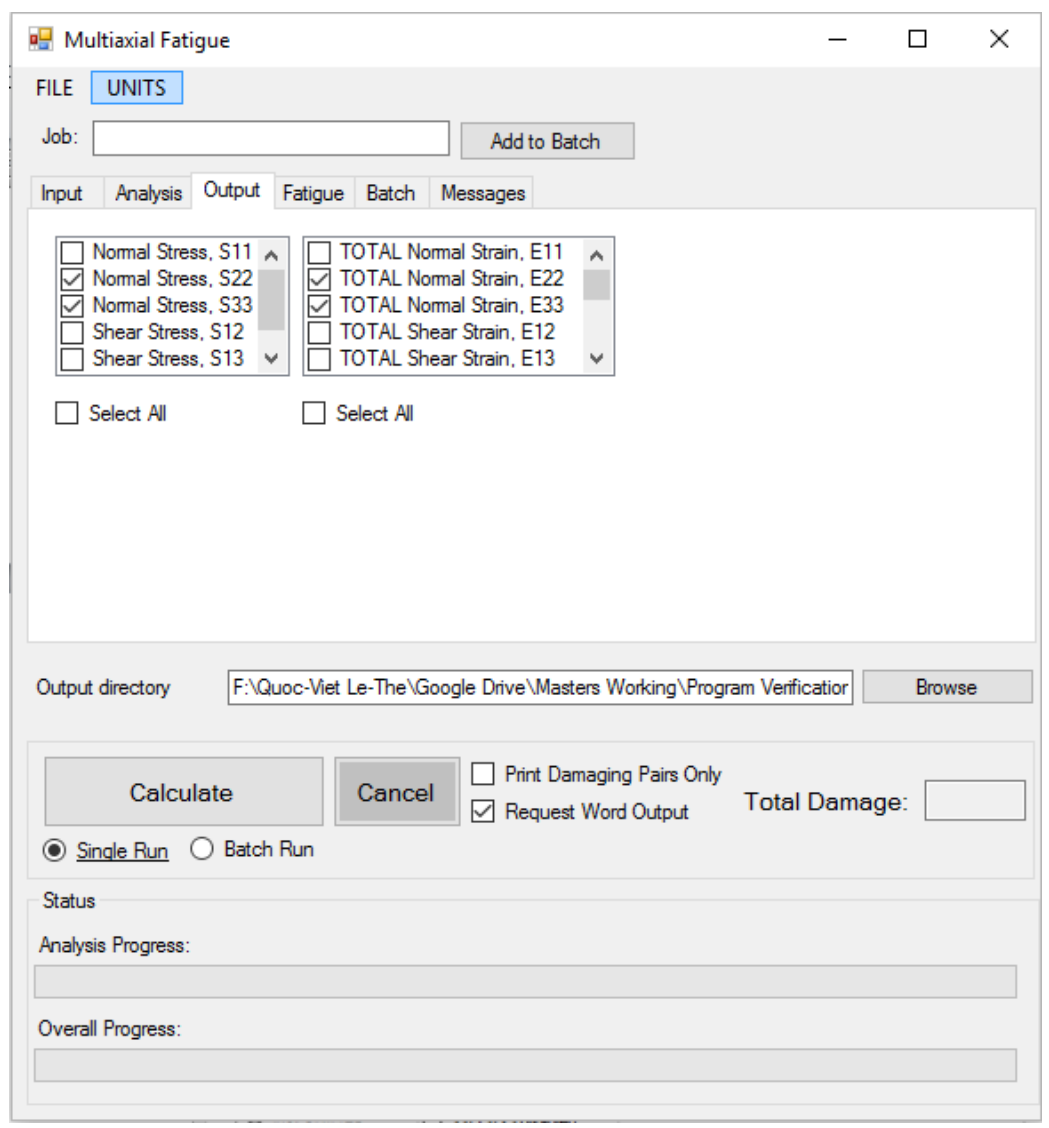

Figure B.3-3: Output request settings. 


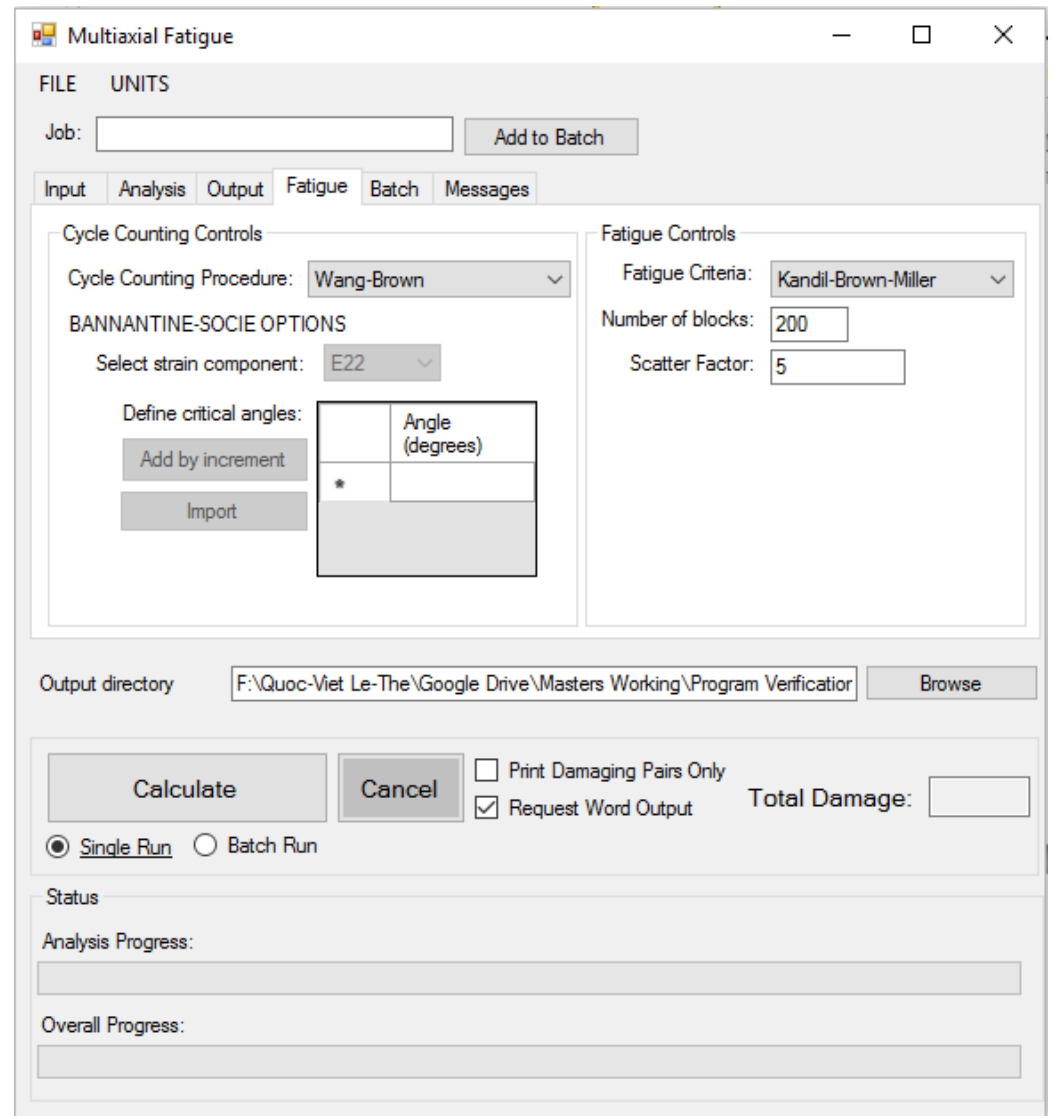

Figure B.3-4: Fatigue settings. 


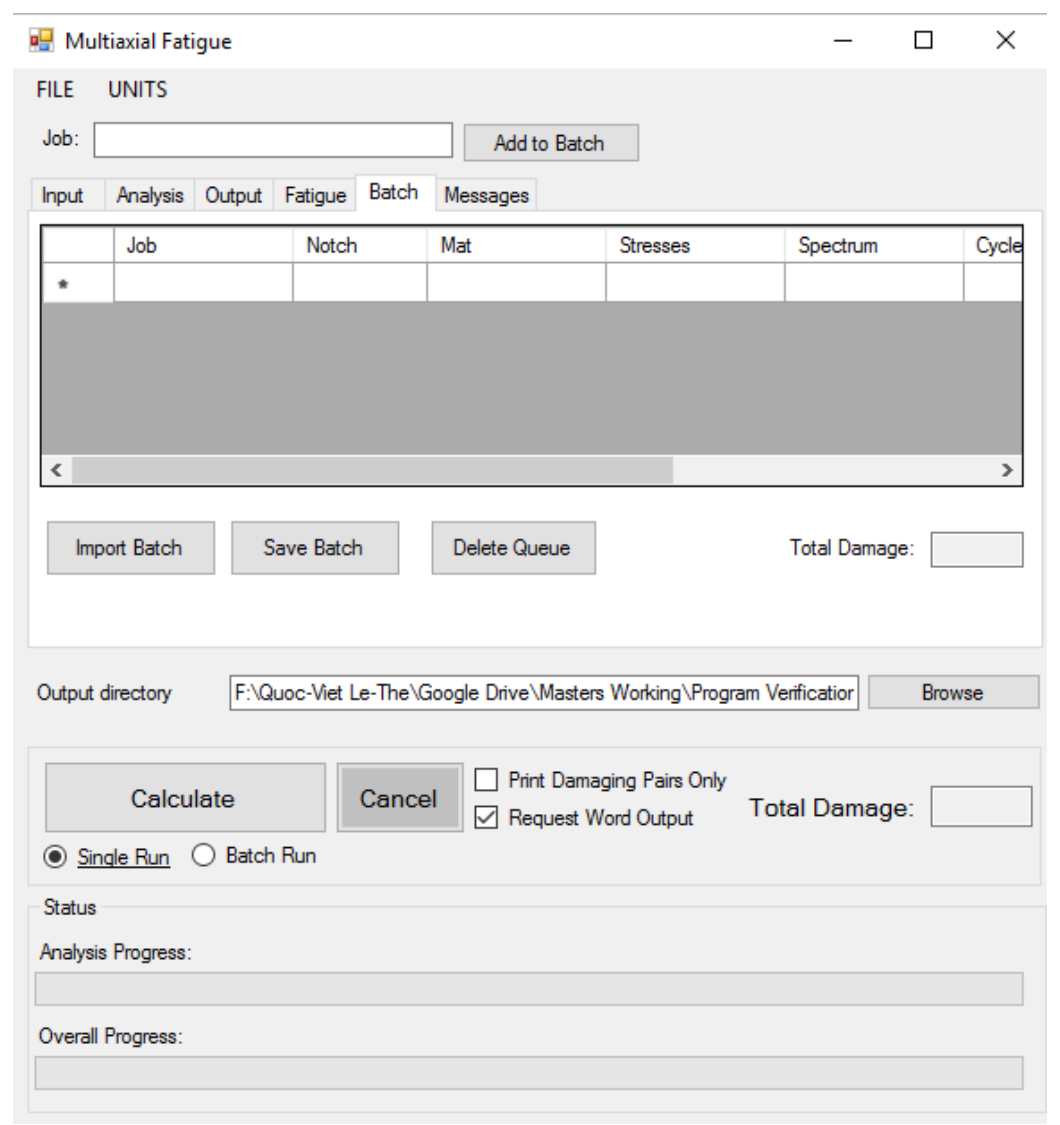

Figure B.3-5: Batch analysis settings.

\section{B.4 Future Improvements}

The program is in its first iteration and has many areas of improvement. Currently a major area of improvement is the generation of the Word output file. For very large analyses, the generation of the Word output file exceeds the duration of the analysis by hours. Methods for more efficient generation of formatted reports can be investigated. In addition to this, there can be more safeguards designed into the program to catch more analytical errors in the analysis and generate more detailed error reports for the user. 